\author{
Universidade de São Paulo \\ Instituto de Física
}

\title{
Análise bimodal de teletransporte de estados quânticos da luz no espaço da frequência
}

\author{
Lucas Nunes Faria
}

Orientador: Prof. Dr. PAULO ALBERTO NUSSENZVEIG

Dissertação de mestrado apresentada ao Instituto de Física da Universidade de São Paulo, como requisito parcial para a obtenção do título de Mestre em Ciências.

Banca Examinadora:

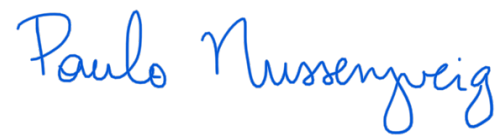

Prof. Dr. Paulo Alberto Nussenzveig - Orientador (USP)

Prof. Dr. Sebastião José Nascimento de Pádua (UFMG)

Prof. Dr. Carlos Eduardo Rodrigues de Souza (UFF) 
FICHA CATALOGRÁFICA

Preparada pelo Serviço de Biblioteca e Informação do Instituto de Física da Universidade de São Paulo

\section{Faria, Lucas Nunes}

Análise bimodal de teletransporte de estados quânticos da luz no espaço da frequência. São Paulo, 2020.

Dissertação (Mestrado) - Universidade de São Paulo. Instituto de Física, Depto. de Física Experimental.

Orientador: Prof. Dr. Paulo Alberto Nussenzveig

Área de Concentração: Ótica quântica, Informação quântica

Unitermos: 1. Física; 2. Física experimental; 3. Mecânica quântica; 4.Óptica quântica. 


\title{
University of São Paulo \\ Physics Institute
}

\section{Two-mode analysis of teleportation of quantum states of light in the frequency domain}

\author{
Lucas Nunes Faria
}

Supervisor: Prof. Dr. PAULO ALBERTO NUSSENZVEIG

Dissertation submitted to the Physics Institute of the University of São Paulo in partial fulfillment of the requirements for the degree of Master of Science.

Examining Committee:

Prof. Dr. Paulo Alberto Nussenzveig - Supervisor (USP)

Prof. Dr. Sebastião José Nascimento de Pádua (UFMG)

Prof. Dr. Carlos Eduardo Rodrigues de Souza (UFF) 



\section{AGRADECIMENTOS}

Começo agradecendo à minha família, em especial aos meus pais, Sidney e Simone. Sem seu incentivo e apoio, esse trabalho nem teria começado.

Agradeço ao meu orientador Paulo Nussenzveig e a meu co-orientador Marcelo Martinelli. Dei muito trabalho nesses dois anos, mas suas orientações esclareceram minhas dúvidas e me impulsionaram sempre para a frente.

Muito obrigado aos membros e ex-membros do LMCAL com quem tive o prazer de interagir: Álvaro, Luiz, Roger, Beatriz, Breno, Emmi, Gabriel, Hans, Igor, Pablo, Pedro, Raul, Rayssa, Renato, Felipe, Yuri e Matheus. Entre eles alguns merecem agradecimentos especiais:

Roger, que coragem sua ter me ajudado a revisar essa dissertação! Você nem fazia parte do time de teletransporte, mas pegou o texto do começo ao fim. Tenho certeza que sem sua ajuda estaria tudo tão mal escrito...

Pedro, nunca vi uma motivação igual a sua. Por mais que você negue, você tem um grande conhecimento do experimento e conseguiu, às 2 da manhã, dar sugestões que avançaram o projeto enormemente. Tenho certeza que você terá um futuro de sucesso aonde for.

Gabriel com sua sede de conhecimento infinita. As discussões com você sempre foram enriquecedoras. Grande coragem a sua de participar da maior parte das medidas da madrugada.

Felipe, você aprende rápido demais! Você eu já conheço há muito mais tempo do que qualquer outro membro do lab e já somos amigos há bastante tempo. Tenho plena confiança de que a continuidade do projeto está em boas mãos com a sua coordenação.

Túlio, eu comecei trabalhando com você. Sua visão experimental é incrível. Confesso que nunca consegui acompanhar seu raciocínio direito, mas você me ajudou muito inclusive depois da minha troca de projetos.

Luiz, não fez parte do time, mas tivemos muitas conversas esclarecedoras sobre física e ainda mais conversas que terminamos sem resposta. E nem estou falando das conversas de mesa de bandejão e de mesa de bar...

Álvaro, meu professor de colombianices. Ótimo experimental e amigo de bebidas. Nunca vou esquecer que era você quem roubava a acetona da minha sala.

Emmi, you didn't stay long but we seemed that we'd known each other since forever. Our minds were always tuned for whatever crazy project arised from the other.

Raul, existe algum assunto sobre o qual a gente já não tenha conversado? Estamos sempre falando sobre tudo que acontece, no sofá do lab, na mesa do bar... Mas um dia, eu quero aprender a dormir tão bem quanto você.

Igor, é para você que esses agradecimentos devem ser os mais especiais. Sei que não é fácil aturar a minha chatice, mas você sempre me ajudou em todas as minhas dúvidas, desde a matemática do teletransporte até a minha curiosidade se eu ia sentir dor se colocasse a mão na mangueira de vácuo.

Vou sentir muito a falta de todos vocês além-mar.

Não posso esquecer dos funcionários do instituto. Agradeço ao Luiz e a Edi, que tanto me ajudaram nas burocracias quando nada mais parecia fazer sentido. Marcos e toda a equipe da mecânica, eu tenho certeza que vocês fazem mágica com os equipamentos para conseguir construir as peças que vocês fazem. Edu e Adriano, que ajuda vocês me deram com a eletrônica que parecia que nunca mais ia funcionar! Ao Korogui, que sempre resolveu os problemas com os computadores. Aos funcionários da $\mathrm{CPG}$, por terem me ajudado em vários problemas burocráticos. E também à Rita, que me ajudou com os produtos químicos.

Também agradeço a todos os amigos de fora do lab que fizeram com que trabalhar no dia seguinte fosse mais fácil. Lufê, Rodrigo, Douglas, Angelo, Thati, Felipe, Amanda, Carol, Henrique, 
Kendi, Otto, Paola, Cata, Ana, Mayara, Jeff, Érika, Steph e tantos outros que não cabem aqui... Espero que não se magoem os que não virem seu nome citado, vocês são muitos, mas não me esqueço de vocês mesmo quando o trabalho aperta e tenho que me afastar um tempo.

Agradeço ao CNPq pelo fomento. 


\section{CONTENTS}

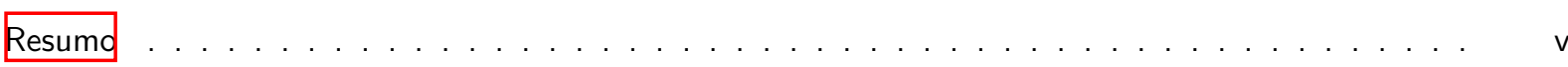

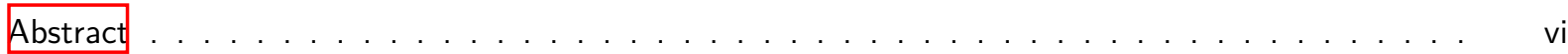

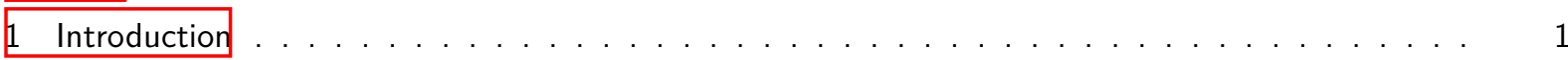

2 Tools for quantum optics . . . . . . . . . . . . . . . . . . . . . . . . . . 3

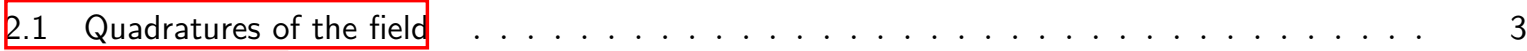

2.2 States of light . . . . . . . . . . . . . . . . . . . . . . . . . 4

2.3 Covariance matrix . . . . . . . . . . . . . . . . . . . . . . . . 5

2.4 Wigner function . . . . . . . . . . . . . . . . . . . . . . . . . 6

2.5 Encoding information in the sidebands . . . . . . . . . . . . . . . . . . 7

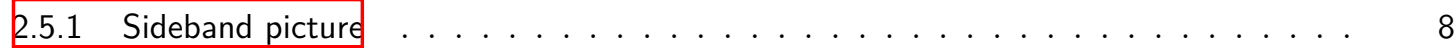

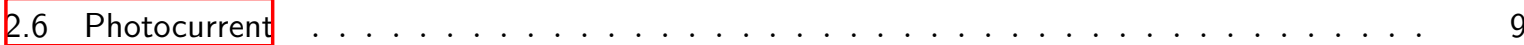

2.6 .1 One-mode and two-mode analysis . . . . . . . . . . . . . . . . 10

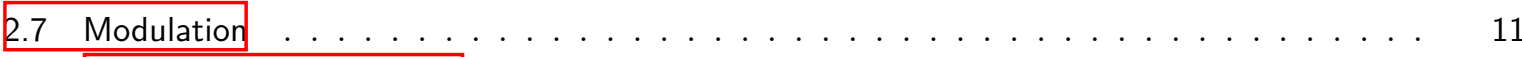

2.7 .1 One-mode analysis . . . . . . . . . . . . . . . . . . . . . 11

2.7 .2 Two-mode analysis . . . . . . . . . . . . . . . . . . . 13

2.8 Opitcal cavities . . . . . . . . . . . . . . . . . . . . . . . . . 14

2.8.1 Preliminary notions . . . . . . . . . . . . . . . . . . . . . 14

2.8.2 Measurement of the field's quadratures . . . . . . . . . . . . . . . . . . . . . 16

2.9 Homodyne and self-homodyne detection . . . . . . . . . . . . . . . . . . 18

2.9 .1 Homodyne detection . . . . . . . . . . . . . . . . . . . . 19

2.9 .2 Self-homodyne detection . . . . . . . . . . . . . . . . . . 19

2.9 .3 Measurement of two beams . . . . . . . . . . . . . . . . . . . . . . 20

3 Entanglement generation in second order non linear media . . . . . . . . . . . . . . . . . . 23

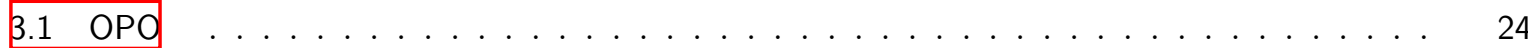

3.1.1 Non linear optics . . . . . . . . . . . . . . . . . . . . . . . 24

3.1.2 Quantum description . . . . . . . . . . . . . . . . . . . . . . 25

3.1.3 Sum and subtraction space . . . . . . . . . . . . . . . . . . 28

3.1.4 Measurement . . . . . . . . . . . . . . . . . . . . . . . . . 29

3.2 Experimental methods . . . . . . . . . . . . . . . . . . . . . . 30

3.2.1 Filter cavity . . . . . . . . . . . . . . . . . . . . 30

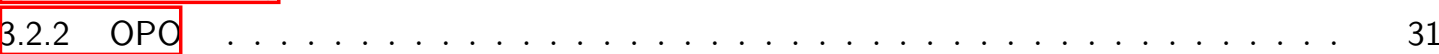

3.2 .3 Input mirror . . . . . . . . . . . . . . . . . . . . . 32

3.2.4 Covariance matrix . . . . . . . . . . . . . . . . . . . . . . 34

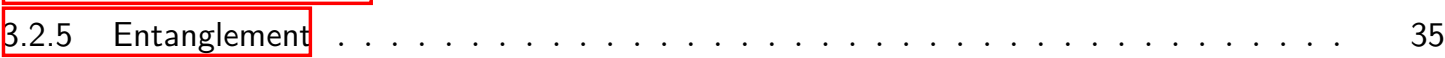

3.2.6 Gray-tracking . . . . . . . . . . . . . . . . . . . . . . 36

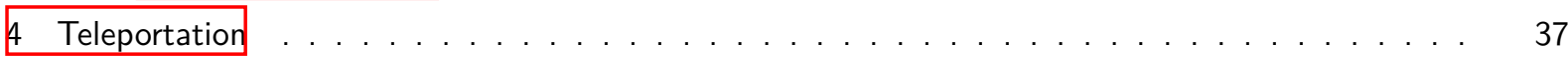

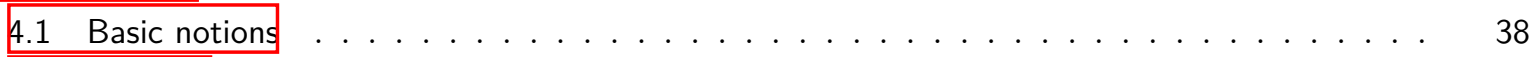

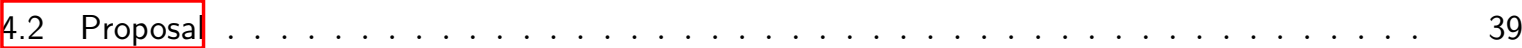

4.2 .1 EPR station . . . . . . . . . . . . . . . . . . . . . . . 40 40

4.2 .2 Victor creates the input state . . . . . . . . . . . . . . . . . . . . . . . 40

4.2 .3 Alice . . . . . . . . . . . . . . . . . . . . . . 42

4.2 .4 Bob ............................... 43

4.2 .5 Victor again . . . . . . . . . . . . . . . . . . . . . . 44

4.3 Gain adjustment . . . . . . . . . . . . . . . . . . . . . . . 45

4.4 Realistic quantum teleportation . . . . . . . . . . . . . . . . . . . 47 
4.4 .1 Finite squeezing . . . . . . . . . . . . . . . . . . . . . . . . . 47

4.4 .2 Detection efficiencies . . . . . . . . . . . . . . . . . . . . . . 49

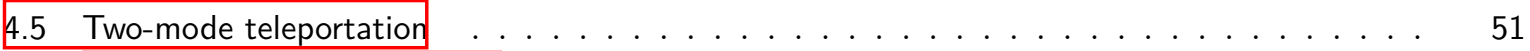

4.5 .1 Alice's measurements . . . . . . . . . . . . . . . . . . . . . . 51

4.5 .2 Victor's measurements . . . . . . . . . . . . . . . . . . . . . 51

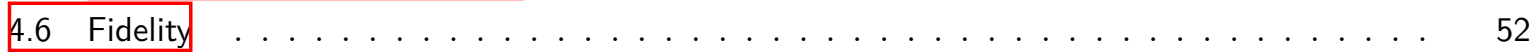

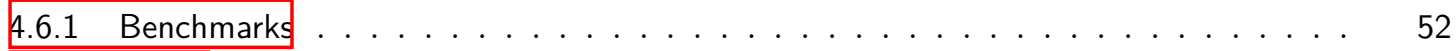

4.6 .2 Losses . . . . . . . . . . . . . . . . . . . . . . . . . 54

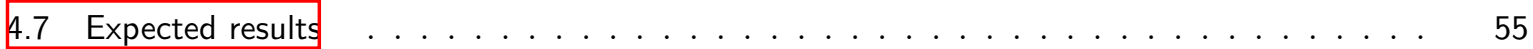

4.7 .1 Classical teleportation . . . . . . . . . . . . . . . . . 55

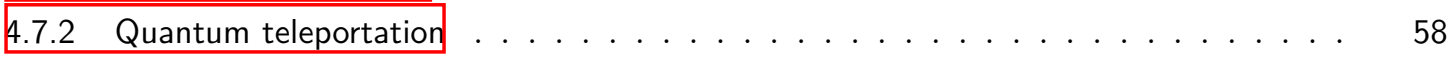

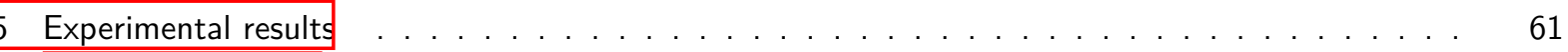

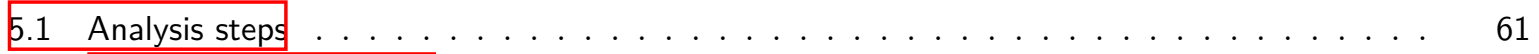

5.1 .1 Gain adjustment . . . . . . . . . . . . . . . . . . . . 61

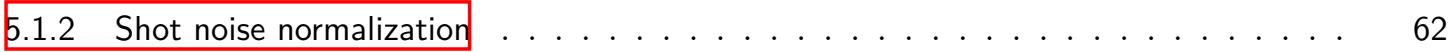

5.1 .3 Teleportation analysis . . . . . . . . . . . . . . . . . 63

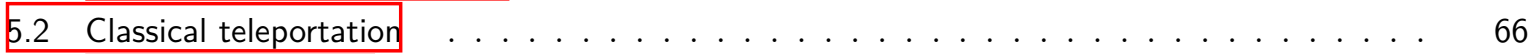

5.2 .1 Mean values . . . . . . . . . . . . . . . . . . . . . . . . . 67

5.2 .2 Variances . . . . . . . . . . . . . . . . . . . . . . . . . . . 69

5.2 .3 Teleportation for the uncorrected input state . . . . . . . . . . . . . 71

5.2 .4 Teleportation for the corrected Input state . . . . . . . . . . . . . . . . 72

5.2 .5 Two-mode analysis . . . . . . . . . . . . . . . . . . . . 73

5.3 Quantum teleportation . . . . . . . . . . . . . . . . . . . . . . . 75

5.3 .1 Comparison between classical an quantum teleportation . . . . . . . . . . . . 76

5.3 .2 Quantum teleportation . . . . . . . . . . . . . . . . . . . . . . . 77

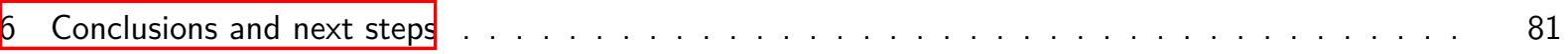

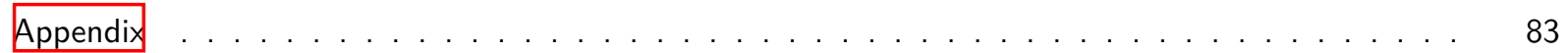

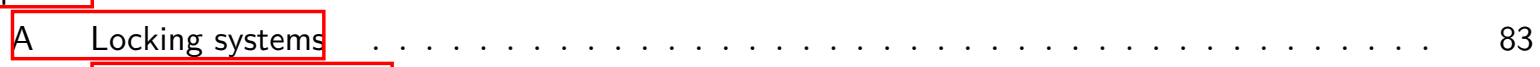

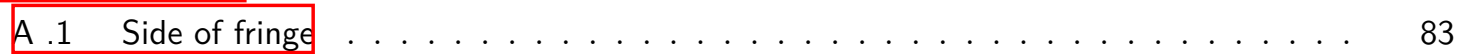

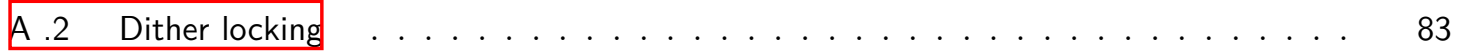

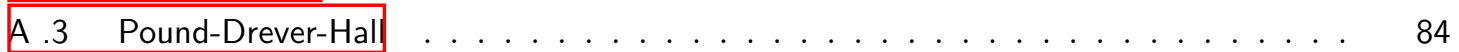

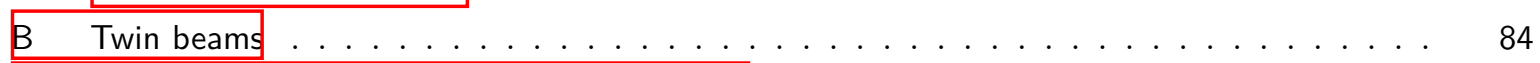

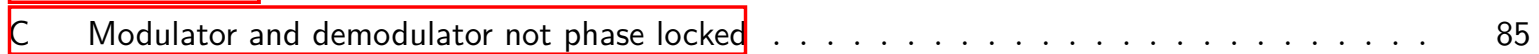

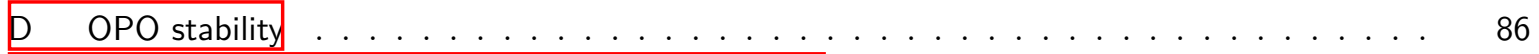

E Classical teleportation results for other input states . . . . . . . . . . . . . . 88

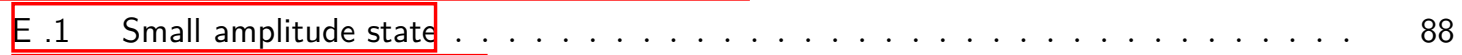

E.2 Asymmetric teleportation . . . . . . . . . . . . . . . . . . . . . 90

References . . . . . . . . . . . . . . . . . . . . . . . . 93 


\section{RESUMO}

\section{Análise bimodal de teletransporte de estados quânticos da luz no espaço da frequência}

A transmissão de informação quântica de uma localidade a outra não é um problema trivial. O teletransporte é uma promessa de solução, um sistema que usa dois canais de comunicação: um clássico - um sistema usual de transmissão —, e um quântico - construído pela geração de emaranhamento entre os estados dos usuários envolvidos. As primeiras realizações deste procedimento davam o resultado correto apenas em uma fração das vezes [1, 2], problema resolvido no ano seguinte no primeiro protocolo de teletransporte incondicional [3]. Desde então, diferentes esquemas experimentais foram sendo implementados e melhores resultados obtidos. O presente trabalho codifica informação nos modos de frequência da luz, com emaranhamento sendo gerado por um Oscilador Paramétrico Ótico acima do limiar [4, 5, 6]. Aqui é desenvolvida a teoria para caracterização de estados em modos de frequência mais gerais, apresentada com os resultados obtidos. Para as perdas existentes no sistema, não é esperado uma fidelidade na comparação entre o estado de saída com o de entrada maior do que $42 \%$ sem o uso de recursos quânticos. O melhor resultado obtido com o uso de feixes emaranhados foi de $40 \%$, perto do limiar da necessidade de recursos quânticos mas sem ultrapassá-lo.

Palavras-chave: Teletransporte, Emaranhamento, Oscilador Paramétrico Ótico. 
ABSTRACT

Two-mode analysis of teleportation of quantum states of light in the frequency domain

Transmission of quantum information from one end to another with high fidelity is not a trivial problem. Teleportation presents itself as promising in this regard, by using two communication channels: a classical one - an usual classical communication system - , and a quantum one - built by sharing entangled sources between the involved parties. The first realizations of this procedure worked on a fraction of the performed trials [1, 2], a problem that was solved one year later with a truly unconditional teleportation protocol [3]. Since then, better results and different experimental schemes have been developed. The present work encodes information in the frequency modes of light beams, with entanglement between two parties being generated by an above-threshold Optical Parametric Oscillator [4, 5, 6]. The theory for characterizing more general frequency mode states is developed here and presented with experimental results. For the present losses in the system, it is expected a fidelity no better than $42 \%$ on the comparison between input and output states without the use of quantum resources. The best measured case with the use of entangled beams resulted in $40 \%$ fidelity, close to the threshold of the need for quantum resources but without overcoming it.

Keywords: Teleportation, Entanglement, Optical Parametric Oscillator. 


\section{INTRODUCTION}

Computation and information processing is part of our everyday lives. Machine learning and neural networks are methods of great interest for better performance in data processing [7]. Although innovative, the cited methods do not make use of the quantum nature of matter, that can also bring novelties to the area. The so called quantum computation and information sciences are promising fields for new development of technologies, with expectations of overcoming computation time of classical algorithms [8].

By exploring the quantum properties of matter in the last decades, suggestions of unique uses of the technology began to emerge. Famously, the proposal of simulating physical systems that are mathematically difficult to solve [9]. The scientific community also saw the arrival of quantum algorithms, such as Shor's [10], providing a solution to the problem of factorization of natural numbers by making use of the superposition of states. Another possibility of the field, more interesting to the theme of this work, are the teleportation protocols such as proposed by [11], exploring the applicability of entanglement.

With such possibilities, one could envision a quantum network consisted of nodes and channels [12] as shown in fig (1.1). A different system capable of storing and processing information is represented by each node, which communicates between each other by means of channels that connect them.

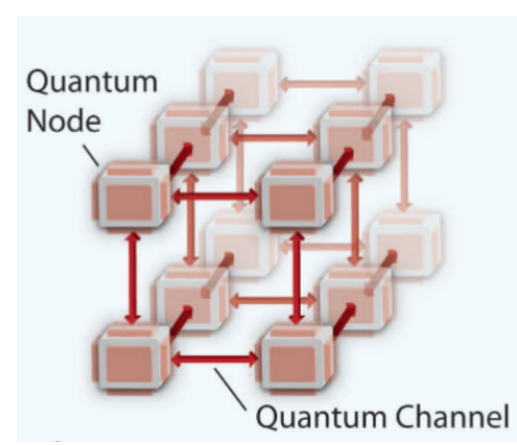

Figure 1.1: Schematic representation of a quantum network. Image from [12].

An experimental implementation of this proposal could, for instance, make use of the known dynamics of interaction between atoms and light. The nodes of the net may be atomic systems, which communicate with other nodes by transmitting the information to modes of light. By means of quantum teleportation of states of light, information can be sent from one node to any other, even if the atomic systems interact with different wavelengths [13]. For it to be possible, it is necessary to build a multicolor entanglement between the light modes.

The work of this thesis fits to the above by studying quantum teleportation of bright beams of light. This is a work on continuous variables, because the parameters of interest may assume values within a continuous spectrum. Information is chosen to be encoded in frequency modes called sidebands. Usually, the instruments capable of imprinting the desired information create two sideband modes with equal energy. A novelty of this work is the expansion to more general states to be used in the scheme. The mathematical basis, as well as physical processes and experimental instruments necessary are described in chapter 2 .

This experiment heavily depends on the careful generation and manipulation of quantum correlations. In this work, the source of entanglement is an optical parametric oscillator (OPO) built with a type II KTP crystal [14]. When pumped by a laser beam, this device generates two entangled beams, called signal and idler. Recent works show that this scheme generates correlations on both sidebands of the three beams of the process (pump, signal and idler) [6]. Chapter 3 is divided in two parts: section 
(3.1) gives the mathematical description of the OPO; and section (3.2) shows details of the experimental implementation along with the auxiliary systems of the device.

Finally, the process of teleportation is presented in chapter 1 with explanations of unique features of this work. It is the connection between the previous chapters. The thesis then leads to chapter 0 , exhibiting experimental results achieved on the system. 


\section{TOOLS FOR QUANTUM OPTICS}

This chapter's goal is to introduce the concepts and set the notation that will be used throughout the entire text. The first four sections present the states on which we will work and give some important mathematical definitions on quantum optics. After the establishment of these tools, the sideband modes we deal with in this teleportation protocol are introduced on section (2.5).

Then, we describe the problem of measurement and how to identify the desired information on the measured electronic signal. The correct analysis of the photocurrent generated by light on a detector allows us to understand how to properly check the success of the teleportation scheme.

The remaining sections are about optical cavities (section (2.8)) and their use (section (2.9)). The latter presents the idea of self-homodyne detection [15, 16], a measurement technique perfected in our group.

\subsection{Quadratures of the field}

It is well known in the literature that light can be described by the oscillation of the electromagnetic fields [17]. During this brief section, we review quantum description of light [18]. The free electric field $\mathbf{E}(\mathbf{x}, t)$ satisfies the wave equation

$$
\nabla^{2} \mathbf{E}(\mathbf{x}, t)-\frac{1}{c^{2}} \frac{\partial^{2}}{\partial t^{2}} \mathbf{E}(\mathbf{x}, t)=0
$$

with the solutions being a sum of different modes $k$

$$
\mathbf{E}(\mathbf{x}, t)=\sum_{k}\left(\alpha_{k}(\mathbf{x}) e^{-i\left(\omega_{k} t+\theta\right)}+\alpha_{k}^{*}(\mathbf{x}) e^{i\left(\omega_{k} t+\theta\right)}\right) \boldsymbol{\epsilon}_{k}
$$

where the polarization vector is represented by $\boldsymbol{\epsilon}_{k}$, the frequency of each mode is $\omega_{k}, \theta$ is a phase term, and $\alpha_{k}(\mathbf{x})$ is the complex amplitude.

The quantization of the electromagnetic field is similar to the treatment given to the quantum harmonic oscillator. The oscillation of energy between electric and magnetic fields is equivalent to the oscillation between potential and kinect energy in the harmonic oscillator [19].

The Hamiltonian of the field describes the stored energy in a given volume

$$
\mathcal{H}=\frac{\epsilon_{0}}{2} \int_{V}\left(|\mathbf{E}(\mathbf{x}, t)|^{2}+c^{2}|\mathbf{B}(\mathbf{x}, t)|^{2}\right) d^{3} V .
$$

By writing

$$
\begin{aligned}
\mathbf{B}(\mathbf{x}, t) & =\sum_{k} \sqrt{\epsilon_{0}} c \omega_{k} p_{k}(t) \mathbf{B}_{k}(\mathbf{x}), \\
\mathbf{E}(\mathbf{x}, t) & =-\sum_{k} \frac{1}{\sqrt{\epsilon_{0}}} q_{k}(t) \mathbf{E}_{k}(\mathbf{x}),
\end{aligned}
$$

we are lead to write the Hamiltonian as

$$
\mathcal{H}=\sum_{k} \frac{1}{2}\left(q_{k}^{2}(t)+\omega^{2} p_{k}^{2}(t)\right) .
$$

This last equation shares a great resemblance with the Hamiltonian for the harmonic oscillator

$$
\mathcal{H}=\sum_{k} \frac{1}{2}\left[\left(\mathbf{p}_{k} / \sqrt{m}\right)^{2}+\omega^{2}\left(\sqrt{m} \mathbf{x}_{k}\right)^{2}\right],
$$

where $p_{k}(t)$ and $q_{k}(t)$ on the electromagnetic Hamiltonian have the same roles as, respectively, $\mathbf{x}_{k}$ and $\mathbf{p}_{k}$ on the harmonic oscillator Hamiltonian. We are able to quantize the electromagnetic field analogously 
to the harmonic oscillator, in terms of the creation and annihilation operators [20]

$$
\hat{H}=\sum_{k} \hbar \omega_{k}\left(\hat{a}_{k}^{\dagger} \hat{a}_{k}+\frac{1}{2}\right),
$$

satisfying

$$
\left[\hat{a}_{i}, \hat{a}_{j}^{\dagger}\right]=\delta_{i j} .
$$

For the harmonic oscillator, these are also called raising and lowering operators. Their effect on the state is to create or destroy one quanta of energy. On the electromagnetic case, this is done by inserting or removing one photon from the system.

This process ends up substituting the complex amplitude $\alpha_{k}$ for the operators $\hat{a}_{k}$

$$
\begin{aligned}
\alpha_{k} & \rightarrow \hat{a}_{k}, \\
\alpha_{k}^{*} & \rightarrow \hat{a}_{k}^{\dagger} .
\end{aligned}
$$

The electric field of light will be written, in the Heisenberg picture, as

$$
\begin{aligned}
\hat{\mathbf{E}}(\mathbf{x}, t) & =\sum_{k}\left(\hat{a}_{k} e^{-i\left(\omega_{k} t+\theta\right)}+\hat{a}_{k}^{\dagger} e^{i\left(\omega_{k} t+\theta\right)}\right) \boldsymbol{\epsilon}_{k} \\
& =\sum_{k}\left[\left(e^{-i \theta} \hat{a}_{k}+e^{i \theta} \hat{a}_{k}^{\dagger}\right) \cos \left(\omega_{k} t\right)+i\left(e^{i \theta} \hat{a}_{k}^{\dagger}-e^{-i \theta} \hat{a}_{k}\right) \sin \left(\omega_{k} t\right)\right] \boldsymbol{\epsilon}_{k} .
\end{aligned}
$$

We are now able to identify the quadratures of the field [21],

$$
\begin{aligned}
& \hat{X}:=e^{-i \theta} \hat{a}_{k}+e^{i \theta} \hat{a}_{k}^{\dagger}, \\
& \hat{Y}:=i\left(e^{i \theta} \hat{a}_{k}^{\dagger}-e^{-i \theta} \hat{a}_{k}\right)
\end{aligned}
$$

or, when $\theta=0$

$$
\begin{aligned}
\hat{p}_{k} & :=\hat{a}_{k}+\hat{a}_{k}^{\dagger}, \\
\hat{q}_{k} & :=i\left(\hat{a}_{k}^{\dagger}-\hat{a}_{k}\right),
\end{aligned}
$$

named, respectively, amplitude and phase quadratures. The annihilation operator can be written in terms of the quadrature operators,

$$
\hat{a}_{k}=\frac{1}{2}\left(\hat{p}_{k}+i \hat{q}_{k}\right) .
$$

Since the quadrature operators are Hermitian, they are observables. Their commutation relation can be obtained from eqn. (2.9),

$$
\left[\hat{p}_{i}, \hat{q}_{j}\right]=2 i \delta_{i j},
$$

which gives us an uncertainty relation for their variances $\Delta \hat{p}_{i}$ and $\Delta \hat{q}_{i}$,

$$
\Delta^{2} \hat{p}_{i} \Delta^{2} \hat{q}_{i} \geq 1 .
$$

When a quadratures' variance equals 1 under this normalization, it is said to be at shot noise level, or standard quantum noise level.

\subsection{States of light}

There are multiple bases on which the state of light can be described [22, 18, 23]. The simplest one is to follow the treatment done to harmonic oscillators and find the eigenvalues of $\hat{n}:=\hat{a}^{\dagger} \hat{a}$, the so called number operator [24, 25]

$$
\hat{n}|n\rangle=n|n\rangle .
$$


The states generated on this manner are called Fock states. It yields an useful basis because its eigenvalues are the number of photons present on the state. However, it is not the best basis for the description of states generated by a laser, which are approximate coherent states. These are the eigenstates of the annihilation operator,

$$
\hat{a}|\alpha\rangle=\alpha|\alpha\rangle
$$

where $\alpha=|\alpha| e^{i \theta}$ are the complex eigenvalues. In the Fock basis, they are represented as

$$
|\alpha\rangle=e^{-\frac{|\alpha|^{2}}{2}} \sum_{n=0}^{\infty} \frac{\alpha^{n}}{\sqrt{n !}}|n\rangle,
$$

an infinite combination of number states. The vacuum state - the state with zero photon on average, $|n\rangle=|0\rangle$ - is also coherent, as can be seen by setting $\alpha=0$ on the above equation. An interesting property of this kind of states is that the mean number of photons and its variance are given by the same number

$$
\langle\alpha|\hat{n}| \alpha\rangle=|\alpha|^{2}=\Delta^{2} \hat{n} .
$$

The quadratures $\hat{p}$ and $\hat{q}$ of coherent states have minimum uncertainty, in the sense that the calculation of eqn (2.17) always results in 1 . Indeed, we have

$$
\Delta^{2} \hat{p}=\Delta^{2} \hat{q}=1
$$

valid for every coherent state. It is said that every quadrature is at shot noise level, also called vacuum noise. Often throughout the text, the measurement of this parameter is going to be used as a scale to assure proper normalization of our data. It is also a threshold for the determination quantum behaviour, as will be seen on chapter 3 .

Coherent states could also be defined by the application of an operator - the displacement operator - on the vacuum state.

$$
\hat{D}(\alpha)|0\rangle=e^{\alpha \hat{a}^{\dagger}-\alpha^{*} \hat{a}}|0\rangle=|\alpha\rangle,
$$

highlighting the fact that coherent states are displaced vacuum states.

Another important class of minimum uncertainty states are the squeezed states, defined by the application of the squeezing operator $\hat{S}(r)$ on a coherent state. A simple particular example is the application of this operator on the vacuum, generating a squeezed vacuum state

$$
\hat{S}(r)|0\rangle=e^{\frac{1}{2} r \hat{a}^{\dagger 2}-\frac{1}{2} r^{*} \hat{a}^{2}}|0\rangle=|r\rangle,
$$

which leads to the variances

$$
\begin{aligned}
\Delta^{2} \hat{p} & =e^{r}, \\
\Delta^{2} \hat{q} & =e^{-r},
\end{aligned}
$$

showing that one quadrature's noise is reduced while the other one is enhanced. Even though the noise of each quadrature is different, computation eqn (2.17) implies squeezed are still minimum uncertainty states, in the sense that the product of the variances results in 1 , the minimun possible value for eqn (2.17). It is by this measurement of noise that entanglemente can be detected and quantified, as will be seen on subsection (3.1.3).

\subsection{Covariance matrix}

The states dealt with on this work are all Gaussian. It means they are fully described by the mean values and variances of the quadratures [26]. A possible way to describe such systems begins by 
grouping the quadrature operators in a vector

$$
\mathbf{x}=\left(\hat{p}_{1}, \hat{q}_{1}, \cdots, \hat{p}_{n}, \hat{q}_{n}\right)^{T} .
$$

The commutation relation between the $i$ 'th and the $j$ 'th terms is given by

$$
\begin{array}{cl}
{\left[\hat{x}_{i}, \hat{x}_{j}\right]} & =2 i \Omega_{i j}, \\
\Omega=\bigoplus \bigoplus^{n} \omega, & \omega=\left(\begin{array}{cc}
0 & 1 \\
-1 & 0
\end{array}\right),
\end{array}
$$

where $\bigoplus$ represents a direct sum. The above equation is nothing more than eqn (2.16) applied on the quadratures in the vector.

The covariance matrix is defined by

$$
V=\frac{1}{2}\left(\left\langle\mathbf{x} \cdot \mathbf{x}^{T}\right\rangle+\left\langle\mathbf{x} \cdot \mathbf{x}^{T}\right\rangle^{T}\right)
$$

Explicitly for a two-mode state,

$$
V=\left(\begin{array}{cccc}
\Delta^{2} \hat{p}_{1} & C\left(\hat{p}_{1}, \hat{q}_{1}\right) & C\left(\hat{p}_{1}, \hat{p}_{2}\right) & C\left(\hat{p}_{1}, \hat{q}_{2}\right) \\
C\left(\hat{q}_{1}, \hat{p}_{1}\right) & \Delta^{2} \hat{q}_{1} & C\left(\hat{q}_{1}, \hat{p}_{2}\right) & C\left(\hat{q}_{1}, \hat{q}_{2}\right) \\
C\left(\hat{p}_{2}, \hat{p}_{1}\right) & C\left(\hat{p}_{2}, \hat{q}_{1}\right) & \Delta^{2} \hat{p}_{2} & C\left(\hat{p}_{2}, \hat{q}_{2}\right) \\
C\left(\hat{q}_{2}, \hat{p}_{1}\right) & C\left(\hat{q}_{2}, \hat{q}_{1}\right) & C\left(\hat{q}_{2}, \hat{p}_{2}\right) & \Delta^{2} \hat{q}_{2}
\end{array}\right),
$$

where the variances of the quadratures are the diagonal elements and all the others are correlations between different quadratures, described by symmetric covariances [16]. The indices 1 and 2 simply denotes the different modes on the system. The correlations between quadratures $X$ and $Y$ are defined by

$$
C(X, Y)=\langle(X-\langle X\rangle)(Y-\langle Y\rangle)\rangle
$$

\subsection{Wigner function}

In this section, the Wigner function is introduced [21, 22]. It was first introduced as a means to link the Schrödinger's wavefunction with a probability distribution in phase space [27]. On this work, it will be used for the calculation of expectation values of observables.

Suppose $\hat{O}$ is an operator which we desire to calculate its properties. The Wigner function for this operator is defined as

$$
W[\hat{O}](\boldsymbol{\alpha})=\int_{C^{n}} \frac{d^{n} \boldsymbol{\lambda}^{n}}{\pi} e^{\boldsymbol{\lambda}^{*} \cdot \boldsymbol{\alpha}-\boldsymbol{\alpha}^{*} \cdot \boldsymbol{\lambda}} \operatorname{Tr}\left[\hat{O} e^{\boldsymbol{\lambda} \hat{a}^{\dagger}-\boldsymbol{\lambda} \hat{a}}\right],
$$

where $\boldsymbol{\lambda}$ and $\boldsymbol{\alpha}$ are $n$-dimensional complex numbers representing positions on phase space. Tr represents the trace operation. This function gives us the the expected value for a measurement when the marginal probability is taken, i.e., it is integrated over $\boldsymbol{\alpha}$.

The Wigner function can be calculated for the density matrix $\hat{\rho}$ of a system. For gaussian states,

$$
W[\hat{\rho}](\mathbf{x})=\frac{\exp \left[-\frac{1}{2}(\mathbf{x}-\overline{\mathbf{x}})^{T} V^{-1}(\mathbf{x}-\overline{\mathbf{x}})\right]}{\pi^{n} \sqrt{\operatorname{det} V}},
$$

where $\mathbf{x}$ and $V$ are defined in eqns (2.27) and (2.31), respectively. The vector $\overline{\mathbf{x}}$ is the mean value of each element in $\mathbf{x}$.

For a single mode state with no correlation between quadratures,

$$
\begin{array}{cc}
\mathbf{x} & =\begin{array}{c}
(\hat{p}, \hat{q})^{T}, \\
V
\end{array}=\left(\begin{array}{cc}
\Delta^{2} \hat{p} & 0 \\
0 & \Delta^{2} \hat{q}
\end{array}\right),
\end{array}
$$


leading to the Wigner function

$$
W[\hat{\rho}](\mathbf{x})=\frac{1}{\pi \sqrt{\Delta^{2} \hat{p} \Delta^{2} \hat{q}}} \exp \left[-\frac{1}{2}\left(\frac{(\hat{p}-\langle\hat{p}\rangle)^{2}}{\Delta^{2} \hat{p}}+\frac{(\hat{q}-\langle\hat{q}\rangle)^{2}}{\Delta^{2} \hat{q}}\right)\right]
$$

The expectation value of an operator $\hat{O}$ when applied to state $\hat{\rho}$ is calculated by

$$
\operatorname{Tr}[\hat{O} \hat{\rho}]
$$

or by the Wigner function

$$
\operatorname{Tr}[\hat{O} \hat{\rho}]=\pi^{n} \int_{C^{n}} d^{n} \boldsymbol{\alpha} W[\hat{O}](\boldsymbol{\alpha}) W[\hat{\rho}](\boldsymbol{\alpha})
$$

This will be specially important for evaluating the success of teleportation on section (4.6).

\subsection{Encoding information in the sidebands}

The laser used on this experiment generates approximately coherent states on a single frequency (called carrier frequency) with a narrow bandwidth around it. Information is carried on this field by means of a modulation (as will be explained on subsection (2.7)). This process adds to the laser two other fields on different frequencies, called sideband modes [28]. Due to experimental limitations (approached on subsections (2.8.2) and (3.1.4) ), only a region of frequencies may be used for the choice of sidebands.

This section will deal with the mathematical description of the sideband space and describe the process of modulation of light beams.

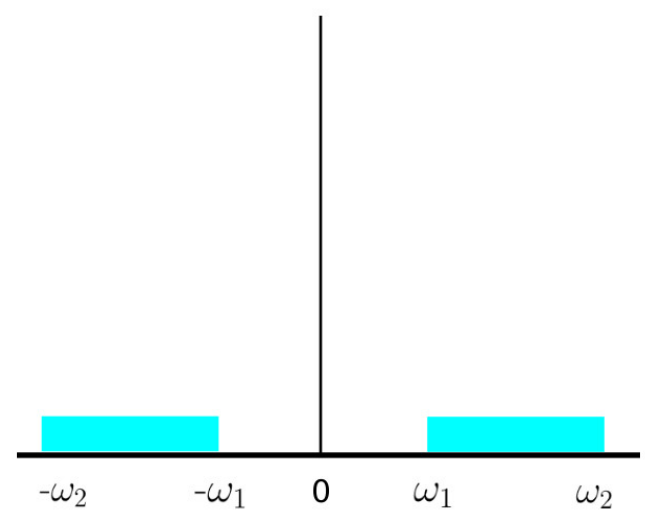

Figure 2.1: Carrier frequency represented by a delta function on 0, accessible sidebands are represented by blue rectangles. 


\subsubsection{Sideband picture}

A bright coherent state can be written as an average value with some fluctuations [29]. This will be expressed on the Heisenbeg picture as

$$
\hat{a}(t)=\alpha+\delta \hat{a}(t)
$$

where new commutation relations come up

$$
\left[\hat{a}(t), \hat{a}^{\dagger}\left(t^{\prime}\right)\right]=\delta\left(t-t^{\prime}\right)
$$

The average value is related to the carrier's mean value of the quadratures $\alpha=\frac{1}{2}(\langle\hat{p}\rangle+i\langle\hat{q}\rangle)$. Since the work is done with bright beams of light, this can be treated as a classical approximation. The quantum fluctuations are represented by the term $\delta \hat{a}(t)=\frac{1}{2}(\delta \hat{p}(t)+i \delta \hat{q}(t))$.

The sideband picture is defined by performing a Fourier transform

$$
\hat{a}(\omega)=\int_{-\infty}^{\infty} \hat{a}(t) e^{i \omega t} d t
$$

leading to a new commutation relation

$$
\left[\hat{a}(\omega), \hat{a}^{\dagger}\left(\omega^{\prime}\right)\right]=\delta\left(\omega-\omega^{\prime}\right)
$$

and definitions for quadrature operators on the frequency domain

$$
\begin{aligned}
\hat{p}(\omega) & =\hat{a}(\omega)+\hat{a}^{\dagger}(\omega), \\
\hat{q}(\omega) & =-i\left(\hat{a}(\omega)-\hat{a}^{\dagger}(\omega)\right), \\
{\left[\hat{p}(\omega), \hat{q}\left(\omega^{\prime}\right)\right] } & =2 i \delta\left(\omega-\omega^{\prime}\right) .
\end{aligned}
$$

The sidebands' information are encoded between two known frequencies $\omega_{1}$ and $\omega_{2}$ as shown on fig (2.1), so the inverse Fourier transform is

$$
\hat{a}(t)=\frac{1}{2 \pi} \int_{-\omega_{1}}^{\omega_{1}} \alpha e^{-i \omega t} \delta(\omega) d \omega+\frac{1}{2 \pi} \int_{-\omega_{2}}^{-\omega_{1}} \hat{a}(\omega) e^{-i \omega t} d \omega+\frac{1}{2 \pi} \int_{\omega_{1}}^{\omega_{2}} \hat{a}(\omega) e^{-i \omega t} d \omega .
$$

And by the above equation, it is easy to see the contribution from the carrier (single frequency located, leading to the factor $\delta(\omega))$, and the lower $\left(\hat{a}_{-}\right)$and upper sidebands $\left(\hat{a}_{+}\right)$

$$
\hat{a}(t)=\alpha+\hat{a}_{-}(t)+\hat{a}_{+}(t)
$$

The results of measurements on the lab are related to the intensity of light, and not to the amplitude.

$$
\begin{aligned}
\hat{I}(t) & =\hat{a}^{\dagger}(t) \hat{a}(t) \\
& =\left(\alpha^{*}+\delta \hat{a}^{\dagger}(t)\right)(\alpha+\delta \hat{a}(t)) \\
& \approx|\alpha|^{2}+|\alpha|\left(e^{i \theta} \delta \hat{a}^{\dagger}(t)+e^{-i \theta} \delta \hat{a}(t)\right),
\end{aligned}
$$

where, on the last line, it was written $\alpha=|\alpha| e^{i \theta}$ and second order fluctuations were neglected. The above equation shows us that the intensity has an average value term $\langle\hat{I}(t)\rangle=|\alpha|^{2}$, hereafter called $D C$ term (direct current). It is common to name $\delta \hat{I}(t)=e^{i \theta} \delta \hat{a}^{\dagger}(t)+e^{-i \theta} \delta \hat{a}(t)$ as $H F$ term (high frequency), which is amplified by $|\alpha|$ on the act of measurement and exhibits the sidebands' information. If $\theta=0$, $\delta \hat{I}(t)$ is related to the fluctuations of $\hat{p}_{k}$ (to be compared with eqn (2.13)); if $\theta=\pi / 2$, it is related to the fluctuations of $\hat{q}_{k}$ (eqn (2.14)); and if $\theta$ is an arbitrary number, the measurement is related to the fluctuations of a combination of the quadratures. This allows us to measure the noise present on each 
quadrature if we are able to control the parameter $\theta$. This is done on this experiment by means of an optical cavity (section (2.8)).

Fig (2.2) is a pictorial representation of a state in the phase space, showing $\hat{p}$ fluctuations parallel to the state vector and $\hat{q}$ fluctuations perpendicular to it. Axis $X$ and $Y$ are defined by writing the electric field as $E(\mathbf{x}, t)=\alpha[X(\mathbf{x}, t) \cos (\omega t)+Y(\mathbf{x}, t) \sin (\omega t)]$ [28].

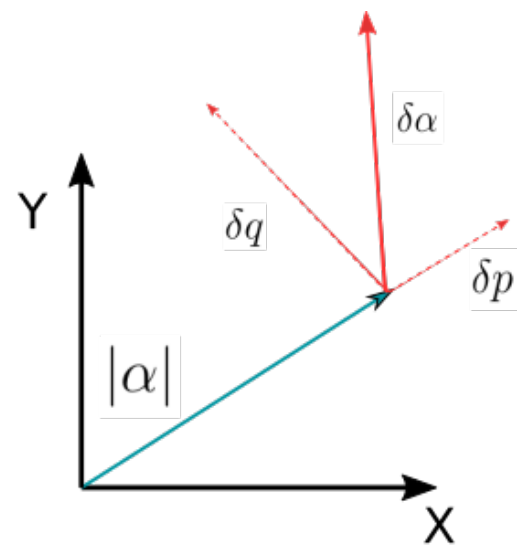

Figure 2.2: The state $\alpha$ in phase space with the quadrature fluctuations.

\subsection{Photocurrent}

On this section we present more details about the measuring process of the sidebands. Beginning with eqn (2.49), the sidebands' related term is given by

$$
\delta \hat{I}(t)=e^{i \theta} \delta \hat{a}^{\dagger}(t)+e^{-i \theta} \delta \hat{a}(t)
$$

Since the encoded information is on a specific frequency (eqn (2.42)), it is interesting to write

$$
\begin{gathered}
\delta \hat{a}(t)=\int_{0}^{\infty} d \omega\left[e^{-i \omega t} \delta \hat{a}(\omega)+e^{i \omega t} \delta \hat{a}(-\omega)\right], \\
\delta \hat{a}^{\dagger}(t)=\int_{0}^{\infty} d \omega\left[e^{i \omega t} \delta \hat{a}^{\dagger}(\omega)+e^{-i \omega t} \delta \hat{a}^{\dagger}(-\omega)\right],
\end{gathered}
$$

which leads to

$$
\delta \hat{I}(t)=\int_{0}^{\infty} d \omega\left[e^{-i \omega t}\left(e^{-i \theta} \hat{a}_{\omega}+e^{i \theta} \hat{a}_{-\omega}^{\dagger}\right)+e^{i \omega t}\left(e^{-i \theta} \hat{a}_{-\omega}+e^{i \theta} \hat{a}_{\omega}^{\dagger}\right)\right]
$$

where $\hat{a}_{\omega}$ designates $\delta \hat{a}(\omega)$ to simplify the notation. The above equation is proportional to the sum of the resulting photocurrent for every frequency. In order to measure only the frequency of interest, an electronic signal of frequency $\Omega$ (the frequency of the sideband being measured) must be mixed with the photocurrent in a process called demodulation [30]. This signal is called electronical local oscillator and may be written as

$$
I_{L O}(t)=I_{0} e^{i \Omega t}+I_{0}^{*} e^{-i \Omega t}
$$

The mix is performed mathematically simply by a multiplication of the last two equations,

$$
\begin{aligned}
\hat{I}_{\text {mix }}(t)=\quad & I_{0} \int_{0}^{\infty} d \omega\left[e^{-i(\omega-\Omega) t}\left(e^{-i \theta} \hat{a}_{\omega}+e^{i \theta} \hat{a}_{-\omega}^{\dagger}\right)+e^{i(\omega+\Omega) t}\left(e^{-i \theta} \hat{a}_{-\omega}+e^{i \theta} \hat{a}_{\omega}^{\dagger}\right)\right] \\
+ & I_{0}^{*} \int_{0}^{\infty} d \omega\left[e^{-i(\omega+\Omega) t}\left(e^{-i \theta} \hat{a}_{\omega}+e^{i \theta} \hat{a}_{-\omega}^{\dagger}\right)+e^{i(\omega-\Omega) t}\left(e^{-i \theta} \hat{a}_{-\omega}+e^{i \theta} \hat{a}_{\omega}^{\dagger}\right)\right],
\end{aligned}
$$


this equation is then averaged to obtain to spectral component

$$
\begin{gathered}
\int_{-\infty}^{\infty} d t \hat{I}_{\text {mix }}(t)=\quad I_{0} \int_{0}^{\infty} d \omega\left[\delta(\omega-\Omega)\left(e^{-i \theta} \hat{a}_{\omega}+e^{i \theta} \hat{a}_{-\omega}^{\dagger}\right)+\delta(\omega+\Omega)\left(e^{-i \theta} \hat{a}_{-\omega}+e^{i \theta} \hat{a}_{\omega}^{\dagger}\right)\right] \\
+I_{0}^{*} \int_{0}^{\infty} d \omega\left[\delta(\omega+\Omega)\left(e^{-i \theta} \hat{a}_{\omega}+e^{i \theta} \hat{a}_{-\omega}^{\dagger}\right)+\delta(\omega-\Omega)\left(e^{-i \theta} \hat{a}_{-\omega}+e^{i \theta} \hat{a}_{\omega}^{\dagger}\right)\right] \\
=I_{0}\left(e^{-i \theta} \hat{a}_{\Omega}+e^{i \theta} \hat{a}_{-\Omega}^{\dagger}\right)+I_{0}^{*}\left(e^{-i \theta} \hat{a}_{-\Omega}+e^{i \theta} \hat{a}_{\Omega}^{\dagger}\right) .
\end{gathered}
$$

$I_{0}$ can be separated on its real and imaginary parts $I_{0}=|I|(\cos \phi+i \sin \phi)$.

$$
\begin{aligned}
\hat{I}_{\text {out }}=\quad & |I|\left[\cos \phi\left(e^{-i \theta} \hat{a}_{\Omega}+e^{i \theta} \hat{a}_{-\Omega}^{\dagger}+e^{-i \theta} \hat{a}_{-\Omega}+e^{i \theta} \hat{a}_{\Omega}^{\dagger}\right)\right. \\
& \left.+i \sin \phi\left(e^{-i \theta} \hat{a}_{\Omega}+e^{i \theta} \hat{a}_{-\Omega}^{\dagger}-e^{-i \theta} \hat{a}_{-\Omega}-e^{i \theta} \hat{a}_{\Omega}^{\dagger}\right)\right] \\
=\quad & |I|\left\{\cos \phi\left[\cos \theta\left(\hat{p}_{\Omega}+\hat{p}_{-\Omega}\right)+\sin \theta\left(\hat{q}_{\Omega}+\hat{q}_{-\Omega}\right)\right]\right. \\
& \left.+\sin \phi\left[\cos \theta\left(\hat{q}_{-\Omega}-\hat{q}_{\Omega}\right)-\sin \theta\left(\hat{p}_{-\Omega}-\hat{p}_{\Omega}\right)\right]\right\},
\end{aligned}
$$

where the quadrature definitions (eqns (2.13) and (2.14)) where used on the last passage.

The resulting equation depends on two angle parameters: $\theta$ and $\phi$. $\theta$ is optical and, as it will be seen on section (2.9), can be controlled with the help of an optical cavity or homodyne detection. $\phi$ is electronic, a change on this term is a change on the phase of the local electric oscillator signal used for the mixture.

Both demodulation quadratures (the signal proportional to $\sin \phi$ and the signal proportional to $\cos \phi)$ are Hermitian operators that can be separated and measured individually [15].

$$
\begin{aligned}
& \hat{I}_{\cos }=\frac{\hat{p}_{s}}{\sqrt{2}} \cos \theta+\frac{\hat{q}_{s}}{\sqrt{2}} \sin \theta, \\
& \hat{I}_{\mathrm{sin}}=\frac{\hat{q}_{a}}{\sqrt{2}} \cos \theta-\frac{\hat{p}_{a}}{\sqrt{2}} \sin \theta,
\end{aligned}
$$

where

$$
\begin{aligned}
\hat{p}_{s / a} & =\frac{\hat{p}_{\Omega} \pm \hat{p}_{-\Omega}}{\sqrt{2}}, \\
\hat{q}_{s / a} & =\frac{\hat{q}_{\Omega} \pm \hat{q}_{-\Omega}}{\sqrt{2}}
\end{aligned}
$$

representing the symmetric and antisymmetric combination of sidebands' quadratures, that will be used as a basis of the space on this work (subsection (4.5)).

\subsubsection{One-mode and two-mode analysis}

On previous works of the group [13, 31], most states dealt with were considered single-mode, presenting information on $\hat{p}$ and $\hat{q}$ quadratures. However, each modulated beam has two sidebands, each with its own $\hat{p}$ and $\hat{q}$ quadrature information, hence leading us to a two-mode interpretation of the state presented on a single beam, first introduced by the group in 2013 [15, 16]. As will be seen on subsection (2.7), a phase or amplitude modulation imposes relations between the quadratures of each sideband, making a single-mode analysis not detrimental.

On this work, an extension for a two-mode analysis is needed because the goal is the teleportation of states with sidebands which are independent from one another. The one-mode analysis from previous works is obtained from eqn (2.50), by writing it as

$$
\hat{I}(t)=\cos \theta \hat{P}_{\Omega}+\sin \theta \hat{Q}_{\Omega}
$$

where the one-mode quadratures $\hat{P}_{\Omega}$ and $\hat{Q}_{\Omega}$ are

$$
\begin{aligned}
& \hat{P}_{\Omega}=\frac{1}{\sqrt{2}}\left(\hat{p}_{s}+i \hat{q}_{a}\right), \\
& \hat{Q}_{\Omega}=\frac{1}{\sqrt{2}}\left(\hat{q}_{s}-i \hat{p}_{a}\right) .
\end{aligned}
$$


From now on, one-mode analysis will be notated by capital letters on quadratures $\hat{P}$ and $\hat{Q}$, while the quadratures on two-mode analysis will be denoted as $\hat{p}$ and $\hat{q}$.

\subsection{Modulation}

This section will introduce the method for adding energy to the sidebands' modes [28]. Discussion on both one-mode and two-mode analysis will be given. Its experimental implementation will also be presented.

\subsubsection{One-mode analysis}

Consider the state associated with the electric field

$$
E^{(+)}=\alpha e^{-i \omega t}
$$

where $E^{(-)}=\left(E^{(+)}\right)^{*}$ and $\mathbf{E}=\left(E^{(-)}+E^{(+)}\right) \boldsymbol{\epsilon}$ is the known equation (2.10) for the electric field with an amplitude $\alpha$. It describes a coherent state with complex amplitude $\alpha$ and frequency $\omega$, while all other frequency terms are populated by vacuum fluctuations.

In order to add photons to the sidebands, the field can be modulated on a frequency $\Omega$ in a process called amplitude modulation, like shown below

$$
(1+M \cos \Omega t) E^{(+)}=\alpha\left[e^{-i \omega t}+\frac{M}{2}\left(e^{-i(\omega-\Omega) t}+e^{-i(\omega+\Omega) t}\right)\right] .
$$

It is easily seen that the beam now possesses two coherent states on the frequencies detuned $\Omega$ from the carrier. The frequency chosen for the modulation is going to define which sidebands are going to be populated.

Using the sideband picture, we could describe the same process by writing

$$
\alpha+\frac{1}{2}(\delta \hat{P}+i \delta \hat{Q}) \rightarrow \alpha+\frac{1}{2}[(\delta \hat{P}+2 M \alpha)+i \delta \hat{Q}] .
$$

The amplitude quadrature, initially on a vacuum states, is transformed as

$$
\delta \hat{P} \rightarrow \delta \hat{P}+2 M \alpha
$$

a coherent state with a mean quadrature value different than zero,

$$
\langle\hat{P}\rangle=2 M \alpha
$$

The same initial state (2.65) could be modulated on its phase instead of on its amplitude.

$$
\begin{array}{rcc}
E^{(+)} e^{i M \cos \Omega t} & =\alpha e^{-i \omega t}(1+i M \cos \Omega t+\ldots) \\
& =\alpha\left[e^{-i \omega t}+\frac{i M}{2}\left(e^{-i(\omega-\Omega) t}+e^{-i(\omega+\Omega) t}\right)\right] .
\end{array}
$$

resulting again in two sidebands populated by the modulation. On the sideband picture, we have

$$
\alpha+\frac{1}{2}[\delta \hat{P}+i(\delta \hat{Q}+2 M \alpha)]
$$

Now, it's the phase quadrature that is a cohererent state with mean value

$$
\langle\hat{Q}\rangle=2 M \alpha .
$$

In our experiment, modulation was created by the use of an electro-optic modulator (EOM). This device consists on a crystal with a voltage dependent refraction index, allowing us to drive the 


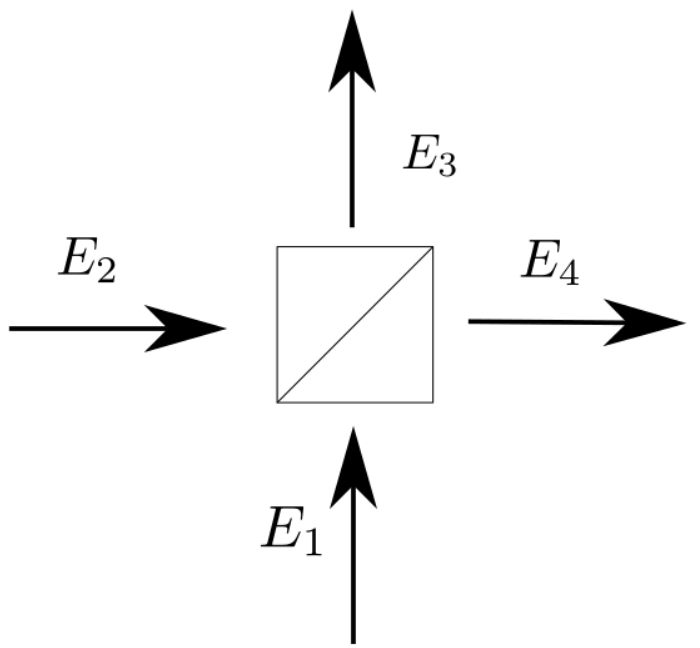

Figure 2.3: Two fields being mixed on a beam splitter.

sidebands' energy in a controlled way [32], effectively acting as the displacement operator of eqn (2.23). The effects described above can be generated by a sinusoidal voltage.

On this experiment, only phase modulation was used. To generate a mean value for other quadratures, we used an interferometer as shown on fig (2.3). A beam splitter is inputted by a phase modulated beam and a a beam with vacuum on the sidebands

$$
\begin{array}{lc}
\hat{a}_{1}= & \alpha_{1}+\frac{1}{2}\left(\delta \hat{P}_{1}+i \delta \hat{Q}_{1}\right), \\
\hat{a}_{2}= & \alpha_{2}+\frac{1}{2}\left[\delta \hat{P}_{2}+i\left(\delta \hat{Q}_{2}+2 M \alpha_{2}\right)\right] .
\end{array}
$$

One of the outputs will be

$$
\hat{a}_{3}=\frac{1}{\sqrt{2}}\left[\alpha_{1}+\alpha_{2} e^{i \theta}+\frac{1}{2}\left(\delta \hat{P}_{1}+\delta \hat{P}_{2} e^{i \theta}+i\left(\delta \hat{Q}_{1}+\delta \hat{Q}_{2} e^{i \theta}+2 M \alpha_{2} e^{i \theta}\right)\right)\right],
$$

where $\theta$ is the phase difference between the two beams. If this parameter is left running, interference fringes can be seen [32]. By setting this parameter at specific values, the modulated quadrature can be changed. When $\theta=0$, the result is

$$
\hat{a}_{3}=\frac{1}{\sqrt{2}}\left[\alpha_{1}+\alpha_{2}+\frac{1}{2}\left[\delta \hat{P}_{1}+\delta \hat{P}_{2}+i\left(\delta \hat{Q}_{1}+\delta \hat{Q}_{2}+2 M \alpha_{2}\right)\right]\right],
$$

a phase modulated state. But when $\theta=\pi / 2$

$$
\hat{a}_{3}=\frac{1}{\sqrt{2}}\left[\alpha_{1}+i \alpha_{2}+\frac{1}{2}\left[\delta \hat{P}_{1}-\delta \hat{Q}_{2}-2 M \alpha_{2}+i\left(\delta \hat{Q}_{1}+\delta \hat{P}_{2}\right)\right]\right],
$$

the result is amplitude modulated. Different values of $\theta$ give rise to states with modulation on both quadratures

$$
\begin{aligned}
\hat{a}_{3}=\frac{1}{\sqrt{2}}\left[\alpha_{1}+\alpha_{2} \cos \theta+i \alpha_{2} \sin \theta\right. & +\frac{1}{2}\left(\delta \hat{P}_{1}+\delta \hat{P}_{2} \cos \theta-\delta \hat{Q}_{2} \sin \theta-2 M \alpha_{2} \sin \theta+\right. \\
& \left.\left.+i\left(\delta \hat{Q}_{1}+\delta \hat{P}_{2} \sin \theta+\delta \hat{Q}_{2} \cos \theta+2 M \alpha_{2} \cos \theta\right)\right)\right] .
\end{aligned}
$$

The resulting mean values of each quadrature are

$$
\begin{gathered}
\left\langle\hat{P}_{3}\right\rangle=-2 M \alpha_{2} \sin \theta, \\
\left\langle\hat{Q}_{3}\right\rangle=2 M \alpha_{2} \cos \theta .
\end{gathered}
$$




\subsubsection{Two-mode analysis}

Equation (2.66), on a more general case, can be written as

$$
(1+M \cos (\Omega t+\phi)) E^{(+)}=\alpha\left[e^{-i \omega t}+\frac{M}{2}\left(e^{i \phi} e^{-i(\omega-\Omega) t}+e^{-i \phi} e^{-i(\omega+\Omega) t}\right)\right],
$$

where $\phi$ takes into account a modulation phase. This equation can be rewritten as

$$
\alpha\left[e^{-i \omega t}+\frac{M}{2}(\cos \phi-i \sin \phi) e^{-i(\omega+\Omega) t}+\frac{M}{2}(\cos \phi+i \sin \phi) e^{-i(\omega-\Omega) t}\right],
$$

separated into terms for each sideband. It can be interpreted as

$$
\hat{a}=\alpha+\frac{1}{2}\left(\hat{p}_{-\Omega}+i \hat{q}_{-\Omega}\right)+\frac{1}{2}\left(\hat{p}_{\Omega}-i \hat{q}_{\Omega}\right)
$$

written in terms of the quadratures of each sideband.

It is interesting to analyse the above equation using the symmetric and antisymmetric combinations of quadratures. We notice a population on

$$
\left\langle\hat{p}_{s}\right\rangle=M \alpha \cos \phi, \quad\left\langle\hat{q}_{a}\right\rangle=M \alpha \sin \phi,
$$

while the others remain on vacuum states. After the discussion of subsection (2.6.1), this result is not unexpected for an amplitude modulation, since there are the combination of quadratures responsible for defining $\hat{P}$ on an one-mode analysis.

Following the same reasoning used for amplitude modulation, a phase modulation is

$$
E^{(+)} e^{i M \cos (\Omega t+\phi)}=\alpha\left[e^{-i \omega t}+\frac{i M}{2}\left(e^{i \phi} e^{-i(\omega-\Omega) t}+e^{-i \phi} e^{-i(\omega+\Omega) t}\right)\right],
$$

rewritten as

$$
\alpha\left[e^{-i \omega t}+\frac{M}{2}(\sin \phi+i \cos \phi) e^{-i(\omega+\Omega) t}+\frac{M}{2}(-\sin \phi+i \cos \phi) e^{-i(\omega-\Omega)}\right] .
$$

An analysis of the combination of sidebands' quadratures on this case yields

$$
\left\langle\hat{p}_{a}\right\rangle=-M \alpha \sin \phi, \quad\left\langle\hat{q}_{s}\right\rangle=M \alpha \cos \phi .
$$

Quadratures associated with $\hat{Q}$ are different than zero while others are null.

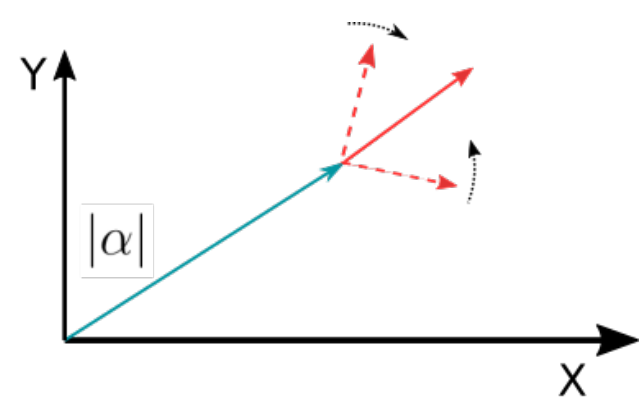

(a) Amplitude modulation.

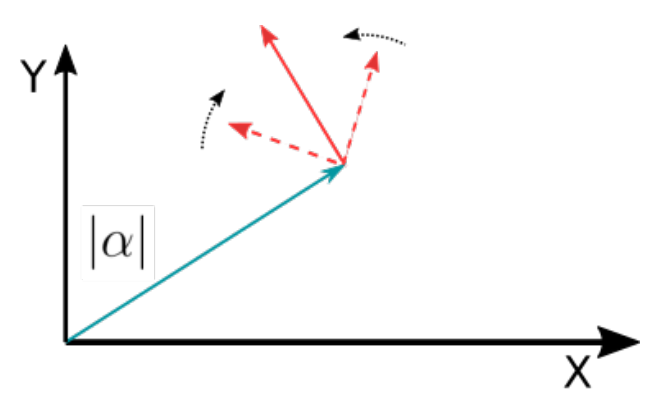

(b) Phase modulation

Figure 2.4: Optical modulations and generated sidebands in dashed lines oscillating on phase space on frequencies $\Omega$ and $-\Omega$ relative to the carrier. Continuous lines represent the vectorial sum of the sidebands' vectors. 
Fig (2.4) suggests a geometric view of the modulation result. In fig (2.4.a) — amplitude modulation - the sidebands vectorial sum always results in a vector aligned with the $\hat{P}$ axis (fig (2.2)) while the sum of each sidebands' $\hat{q}$ quadrature is zero. Fig (b) - phase modulation — depicts the same for opposite quadratures. In order to generate more complex sideband modes, a description in terms of amplitude and phase modulation is not enough. An extra parameter, to depict the phase relation between both modulations, is necessary. The teleportation protocol presented on chapter 1 will require the values of each individual sideband's quadrature.

\subsection{Opitcal cavities}

In quantum optics laboratories, the most common method for measuring the quadratures' information is homodyne detection. This will be briefly presented on next section, as a comparison to the resonator detection method, used on this work, presented on this section. Despite its uncommonness, the measurement of quadratures by optical cavities gives more information about the state [16].

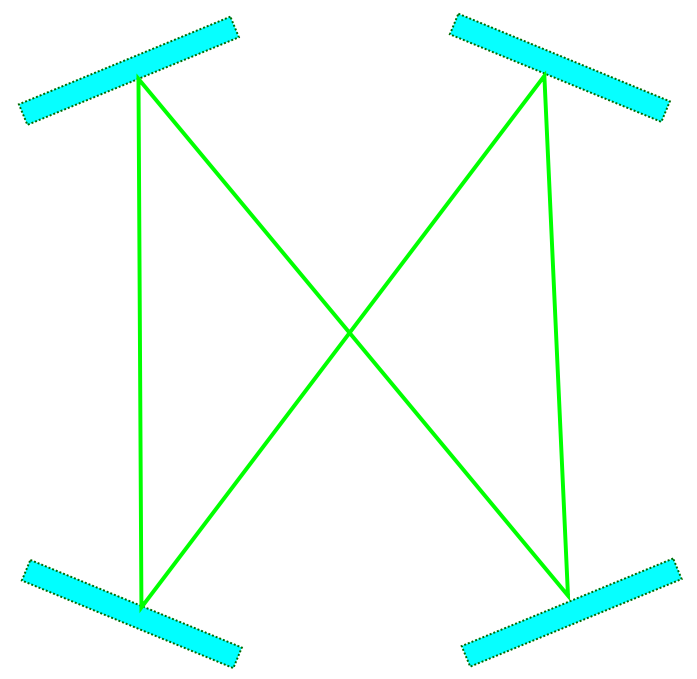

Figure 2.5: Four mirror cavity

Such devices consist of a number of mirrors arranged in a way that light entering the cavity remains inside it for some time before leaving it or being absorbed, as shown on fig (2.5). The transmission of light to the cavity or from it is achieved by using non perfect mirrors.

\subsubsection{Preliminary notions}

A cavity imposes a path for light to travel, a parameter called perimeter. If the perimeter is $L$, the resonant frequencies will be

$$
\omega_{R}=n \frac{c}{L}=n F S R
$$

where $n$ is any integer number, $c$ is the speed of light and $F S R=\frac{c}{L}$ is the free spectral range, defined as the frequency separation between two resonant conditions.

Another important parameter is the finesse, used for measuring the quality of the cavity. It takes into account the losses in the device as explained below. Consider the amplitude of the beam after the $n$ 'th round trip inside the cavity as $\alpha_{n}$, with $\alpha_{0}$ as the incident beam's amplitude. The net result is

$$
\alpha_{c a v}=\sum_{n=0}^{\infty} \alpha_{n} .
$$


The amplitude on each round trip will be attenuated, hence $\alpha_{n}$ is related to $\alpha_{n-1}$ by the loss factor $g$ (usually due to the transmission and absorption mirrors). If the cavity is not on tune (it's perimeter is not related to a resonant frequency), each round trip will gain a phase $\phi=2 \pi \frac{\left(\omega-\omega_{R}\right)}{L}$. This results in

$$
\alpha_{n}=\alpha_{n-1} g e^{i \phi}
$$

which leads to

$$
\alpha_{c a v}=\frac{\alpha_{0}}{1-g e^{i \phi}}
$$

A detector would therefore measure

$$
\begin{aligned}
I_{c a v} & =\frac{\left|\alpha_{0}\right|^{2}}{\left|1-g e^{i \phi}\right|^{2}} \\
& =\frac{\left|\alpha_{0}\right|^{2}}{1+g^{2}-2 g \cos \phi} \\
& =\frac{\left|\alpha_{0}\right|^{2}}{(1-g)^{2}+4 g \sin ^{2} \frac{\phi}{2}} \\
& =\frac{\left[\left|\alpha_{0}\right| /(1-g)\right]^{2}}{1+\left(\frac{2 F}{\pi}\right)^{2} \sin ^{2} \frac{\phi}{2}},
\end{aligned}
$$

where the finesse parameter comes up

$$
F=\frac{\pi \sqrt{g}}{(1-g)}
$$

It is related only to the losses, making it indeed a good parameter for measuring the quality of the cavity.

Another important parameter is the linewidth $\gamma$, it is related with the other two by $\gamma=\frac{F S R}{F}$. It can be better understood when we think about scanning the cavity, that means varying the detuning $\Delta=\left(\omega-\omega_{R}\right) / \gamma$ as the intensity is measured. This is equivalent to varying $\phi$ in eqn (2.92). The linewidth of this curve is given by the width of the lorentizian curve (2.92) at half the peak's height. As fig (2.6) shows, a greater finesse results in a sharper peak and a smallest linewidth.

Experimentally, a piezoelectric material $(P Z T)$ is attached to one of the mirrors. An electric voltage applied to this material changes its size, which in turn changes the perimeter $L$ of the cavity, making it possible to scan the detuning $\Delta$ as described on last paragraph. It is by a controlled manipulation of this electric voltage that the cavity can be locked at specific positions as explained on appendix (A).

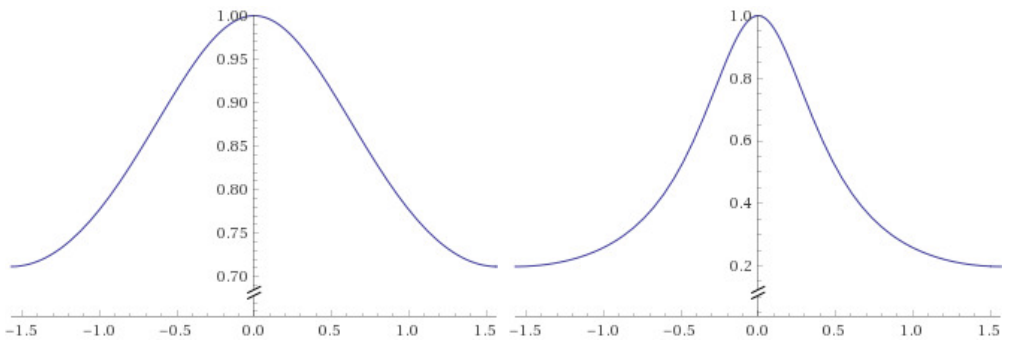

(a) $\mathrm{F}=1$ (b) $\mathrm{F}=10$

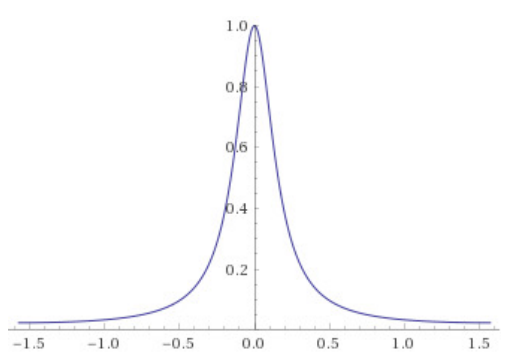

(c) $\mathrm{F}=100$

Figure 2.6: Intensity output for a cavity with different finesses as a function of the detuning. 


\subsubsection{Measurement of the field's quadratures}

In order to study how it can be used for the measurement of quadratures, we need to understand the transformations each component of the field suffers when reflected from a cavity. For different frequency inputs the cavity will exhibit different output behaviours.

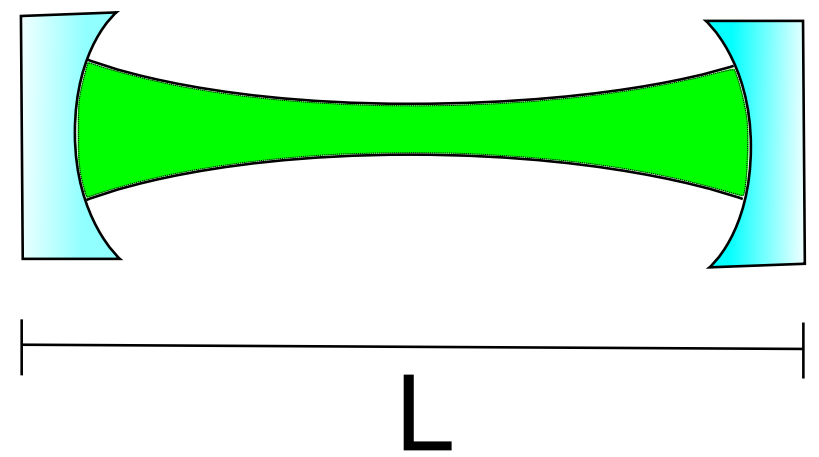

Figure 2.7: Two mirror (Fabry-Perot) cavity.

A two-mirror cavity will be considered here for simplicity. The reflection and transmission coefficients (for the amplitude, not the intensity) for the $i$ 'th mirror are, respectively, $r_{i}$ and $t_{i}$. The overall cavity's reflection and transmission coefficients are [33]

$$
\begin{aligned}
r(\Delta) & =\frac{r_{1}-r_{2} e^{i 2 \pi \Delta / F}}{1-r_{1} r_{2} e^{i 2 \pi \Delta / F},} \\
t(\Delta) & =\frac{t_{1} t_{2} e^{i \pi \Delta / F}}{1-r_{1} r_{2} e^{i 2 \pi \Delta / F}},
\end{aligned}
$$

and the phase of reflected beam will be a function of the detuning as well

$$
e^{i \theta_{R}(\Delta)}=\frac{r(\Delta)}{|r(\Delta)|}
$$

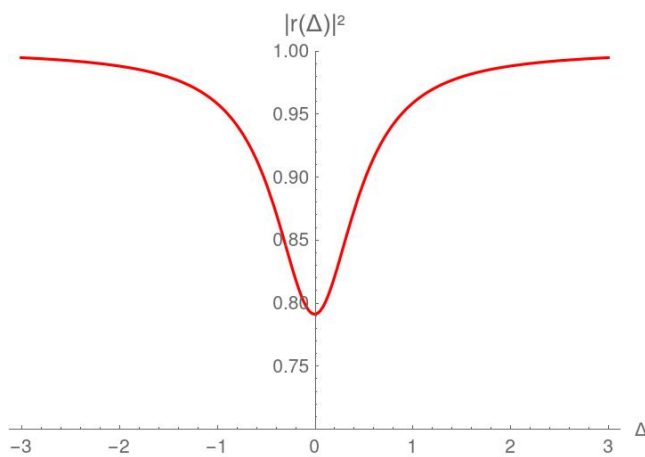

(a) Reflectivity of a cavity. Calculations done with $r_{1}=\sqrt{0.95}$ and $r_{2}=\sqrt{0.997}$, yielding $F=116$.

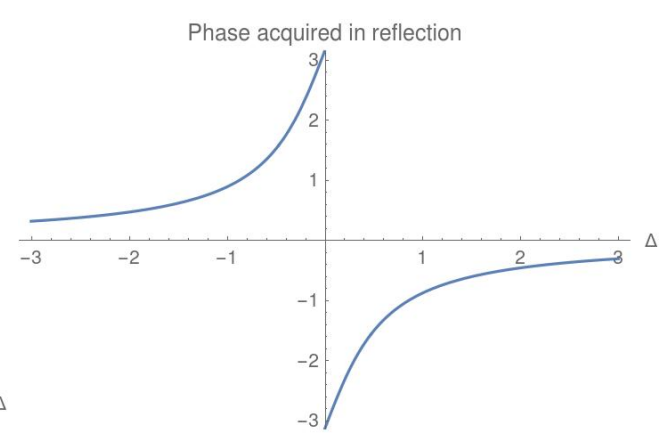

(b) Phase acquired in reflection for the cavity on the same conditions as fig (a). Although the phase seems discontinuous, $e^{i \theta_{R}(\Delta)}$ is not.

Figure 2.8: Intensity output for a cavity with different finesses as a function of the detuning.

The measurement of a beam reflected from a cavity results on the described on section (2.6) if the annihilation operators are replaced by

$$
\hat{a}_{\Omega} \rightarrow r\left(\Delta_{\Omega}\right) \hat{a}_{\Omega}+t\left(\Delta_{\Omega}\right) \hat{b}_{\Omega},
$$

where $\Delta_{\Omega}:=\frac{\Omega-\omega_{R}}{\gamma}$ and $\hat{b}_{\Omega}$ is representing the annihilation operator for the vacuum mode entering the cavity through the other mirror. This change may be applied to check what happens to the $\hat{P}$ quadrature 
of a beam after the reflection from a cavity. By making the above substitution on eqn (2.44) and replacing $\theta$ by $\theta_{R}(\Delta)$, we have $\hat{P}$ as a function of $\Delta$ for each sideband frequency $\nu=\Omega / \gamma$

$$
\begin{aligned}
\hat{P}_{R}[\nu](\Delta)=\quad & \frac{1}{2}\left[\left(e^{-i \theta_{R}(\Delta)} r(\Delta+\nu)+e^{i \theta_{R}(\Delta)} r^{*}(\Delta-\nu)\right) \hat{P}_{i n}(\nu)\right. \\
& +i\left(e^{-i \theta_{R}(\Delta)} r(\Delta+\nu)-e^{i \theta_{R}(\Delta)} r^{*}(\Delta-\nu)\right) \hat{Q}_{i n}(\nu) \\
& +\left(e^{-i \theta_{R}(\Delta)} t(\Delta+\nu)+e^{i \theta_{R}(\Delta)} t^{*}(\Delta-\nu)\right) \hat{P}_{v a c}(\nu) \\
& +i\left(e^{-i \theta_{R}(\Delta)} t(\Delta+\nu)-e^{i \theta_{R}(\Delta)} t^{*}(\Delta-\nu)\right) \hat{Q}_{v a c}(\nu),
\end{aligned}
$$

where $\hat{p}_{R}$ is the reflected quadrature, the subscript in indicates the quadratures of the beam before entenring the cavity and vac the vacuum quadratures. By setting a specific detuning, it is possible to convert the information on $\hat{Q}$ quadrature to the $\hat{P}$ quadrature. The detuning position for this needs to be set at $\Delta \approx 1 / 2[33]$. On the case of this experiment, this is equivalent to locking the cavity when measuring an output intensity of $40 \%$ in relation to the resonance intensity.

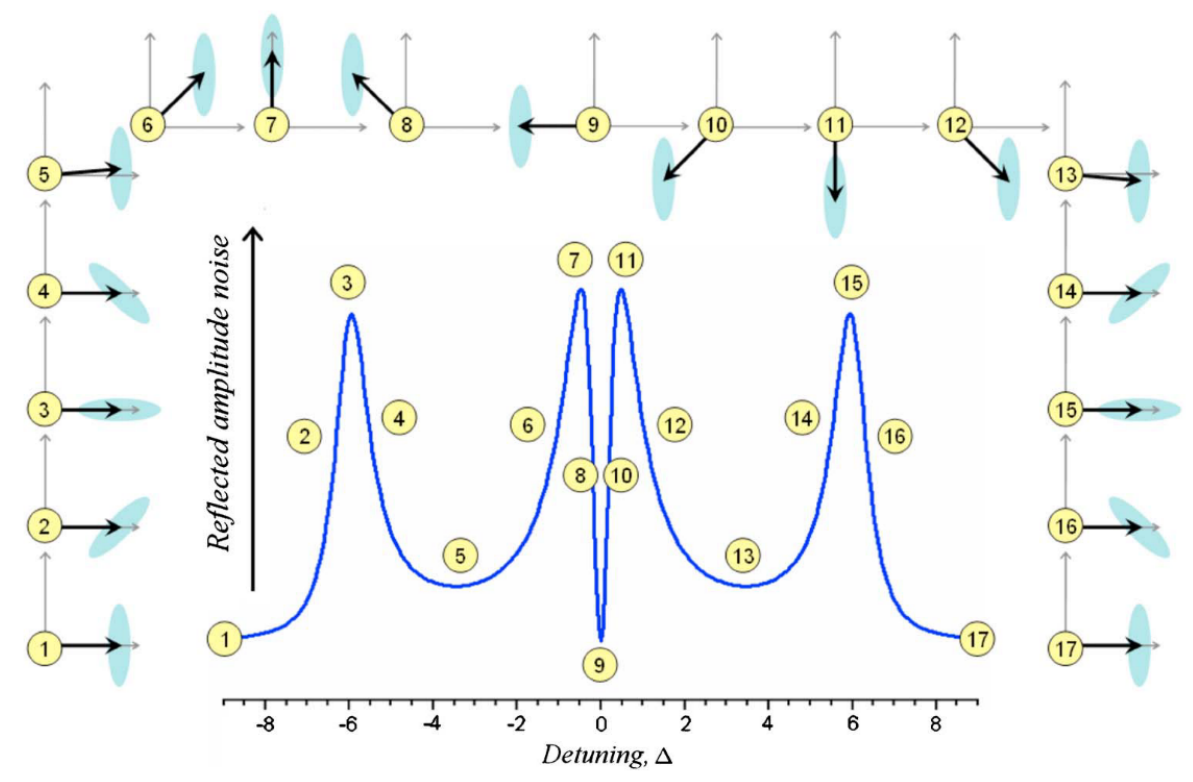

Figure 2.9: Noise power (eqn (2.104)) as ellipse rotates in a detuned cavity. The chosen frequency was 6 times the cavity bandwidth. Image taken from reference [33].

This method is also called rotation of the noise ellipse. If we imagine the phase space of the state, the noises from the quadratures have the form of an ellipse. By the use of a cavity, this ellipse can be rotated and the measured quadrature can be changed at will as shown on fig (2.9). However, this method does not work for measuring sidebands that are too close to the carrier frequency, because the cavity ceases to be able to distinguish the light modes. This problem is avoided by choosing the frequency of the sidebands to be $\Omega \geq \sqrt{2} \gamma[34]$.

We will show the photocurrent result when the cavity is locked at the position of conversion of quadratures $\hat{P} \leftrightarrow \hat{Q}$. This is calculated by applying eqn (2.98) and demodulating the resulting signal as explained on section (2.6). The quadratures of each sideband will have a different phase shift because the cavity has a frequency dependent behaviour. The obtained is

$$
\begin{aligned}
& \hat{I}_{\cos }=\frac{\hat{p}_{a}}{\sqrt{2}} \cos \alpha+\frac{\hat{q}_{s}}{\sqrt{2}} \sin \alpha, \\
& \hat{I}_{\sin }=\frac{\hat{p}_{a}}{\sqrt{2}} \sin \alpha+\frac{\hat{q}_{s}}{\sqrt{2}} \cos \alpha,
\end{aligned}
$$


where

$$
\cos \alpha=\operatorname{Re}\left[r^{*}(\Delta) r(\Delta+\nu)\right], \quad \sin \alpha=\operatorname{Im}\left[r^{*}(\Delta) r(\Delta+\nu)\right]
$$

are terms related to the cavity's reflections for frequency $\nu$ at the detuning $\Delta$ for the quadrature exchange $\hat{P} \leftrightarrow \hat{Q}$. On the above equations, $\operatorname{Re}[X]$ and $\operatorname{Im}[X]$ represent, respectively, the real and imaginary parts of $X$.

If there is no cavity, the modulated results of measuring the beam's $\hat{P}$ quadrature are

$$
\hat{I}_{\cos }=\frac{\hat{p}_{s}}{\sqrt{2}}, \quad \hat{I}_{\sin }=\frac{\hat{q}_{a}}{\sqrt{2}} .
$$

\subsection{Homodyne and self-homodyne detection}

The autocorrelation function for a signal $\hat{I}(t)$ describes the correlation between itself on two different time instants $t$ and $t^{\prime}$ and is defined by

$$
g\left(t, t^{\prime}\right)=\left\langle\hat{I}(t) \hat{I}\left(t^{\prime}\right)\right\rangle
$$

If this function depends only on the difference of times $\tau=t-t^{\prime}$, the signal $\hat{I}(t)$ is said to be stationary. As a consequence, statistical properties of $\hat{I}$ as mean value and variance is going to be constant for every measurement time. And more important, the Wiener-Kinchine theorem [35] allows us to calculate the spectral noise power of the signal by taking a Fourier transform

$$
S(\Omega)=\left\langle\hat{I}_{\Omega} \hat{I}_{-\Omega}\right\rangle
$$

The property $\left\langle\hat{I}_{\Omega} \hat{I}_{\Omega^{\prime}}\right\rangle=0$ holds for every $\Omega^{\prime} \neq-\Omega$.

The stationarity condition cited above is true when measuring the noise present on the quadratures. A measure of the spectral noise power allows us to obtain the desired noise information of the sidebands, enabling us to write the covariance matrix (eqn (2.31)) both for the $s / a$ basis (eqns (2.60) and (2.61)) as for the $\Omega /-\Omega$ basis (eqns (2.44) and (2.45))

$$
\begin{aligned}
V_{s / a} & =\left(\begin{array}{cccc}
\alpha & \gamma & \delta & 0 \\
\gamma & \beta & 0 & \delta \\
\delta & 0 & \beta & -\gamma \\
0 & \delta & -\gamma & \alpha
\end{array}\right), \\
V_{\Omega /-\Omega} & =\left(\begin{array}{cccc}
\alpha^{\prime} & 0 & \gamma^{\prime} & \delta^{\prime} \\
0 & \alpha^{\prime} & \delta^{\prime} & -\gamma^{\prime} \\
\gamma^{\prime} & \delta^{\prime} & \beta^{\prime} & 0 \\
\delta^{\prime} & -\gamma^{\prime} & 0 & \beta^{\prime}
\end{array}\right) .
\end{aligned}
$$

It is interesting to have an intuitive interpretation of the terms on the matrix (2.105). It is possible to identify $\alpha$ and $\beta$ as related to the variances of the $\hat{P}$ and $\hat{Q}$ quadratures, respectively. A geometric vision can be given to $\gamma$ when one looks at figure (2.9), it is related to the tilt of the noise ellipse axis in relation to the carrier vector in phase space. The remaining term, $\delta$, is the energy difference in sidebands's noise. It is required resonator detection scheme for the access of this term since standard homodyne detection cannot. The end of this chapter is dedicated to explaining the differences of both measurement techniques in terms of reconstruction of the exposed terms.

It must be cited, however, that the experiment of this thesis is unable to measure the term $\delta$, even though resonator detection is used (more information on subsection (2.9.2)). The reason for this 
is the high reflectivity of our cavity, which leads to low precision on the measurement of this term [16]. Close to a resonance

$$
r\left(\Delta_{\Omega}\right)=-\frac{d+i 2 \Delta_{\Omega}}{1-i 2 \Delta_{\Omega}}
$$

where $d=|r(0)|$ acts as an impedance matching parameter. The resonater is impedance matched when $d=0$, resulting in a complete substitution of the reflected resonant mode for the transmitted vacuum state. A lossless resonator $d=1$ simply acts as a phase shifter, and homodyne and self-homodyne detection becomes equivalent.

\subsubsection{Homodyne detection}

This technique is performed by mixing the input beam (the one that carries the state that one wants to measure) with a stronger same frequency beam with a controllable phase. The interference between these modes carries the desired information [5].

Consider $\hat{a}$ as the input, containing the information to be measured. The strong field to be mixed with it is called optical local oscillator and it will be represented as a classical field $A=|A| e^{i \theta}$, due to its high intensity compared to the input state. On a 50:50 beam splitter, the outputs of this interference will be

$$
\begin{aligned}
& \hat{c}=\frac{\hat{a}+A}{\sqrt{2}}, \\
& \hat{d}=\frac{\hat{a}-A}{\sqrt{2}} .
\end{aligned}
$$

By subtracting the intensities, we are left with

$$
\hat{I}=\hat{c}^{\dagger} \hat{c}-\hat{d}^{\dagger} \hat{d}=A^{*} \hat{a}+A \hat{a}^{\dagger}=|A|\left(e^{-i \theta} \hat{a}+e^{i \theta} \hat{a}^{\dagger}\right),
$$

so the result is the same as we had before in eqn (2.50), this time amplified by the local oscillator amplitude $A$.

The spectral noise power in this case is

$$
\begin{array}{cc}
S(\Omega)= & \left\langle I_{\Omega} I_{-\Omega}\right\rangle=\frac{1}{2}\left(\left\langle\hat{I}_{\cos }^{2}\right\rangle+\left\langle\hat{I}_{\sin }^{2}\right\rangle\right) \\
= & \frac{\Delta^{2} \hat{p}_{s}+\Delta^{2} \hat{q}_{a}}{2} \cos ^{2} \theta+\frac{\Delta^{2} \hat{q}_{s}+\Delta^{2} \hat{p}_{a}}{2} \sin ^{2} \theta+\left[C\left(\hat{p}_{s}, \hat{q}_{s}\right)-C\left(\hat{p}_{a}, \hat{q}_{a}\right)\right] \sin \theta \cos \theta \\
& \alpha \cos ^{2} \theta+\beta \sin ^{2} \theta+\gamma \sin 2 \theta,
\end{array}
$$

where on the last passage, $\alpha, \beta$ and $\gamma$ are the same terms of the matrix in eqn (2.105). The spectral noise power for a specific frequency is now a function of the phase of the local oscillator. By scanning it, it is possible to measure the terms of the covariance matrix with the exception of $\delta=C\left(\hat{p}_{s}, \hat{p}_{a}\right)=C\left(\hat{q}_{s}, \hat{q}_{a}\right)=$ $\Delta^{2} \hat{p}_{\Omega}+\Delta^{2} \hat{q}_{\Omega}-\Delta^{2} \hat{p}_{-\Omega}-\Delta^{2} \hat{q}_{-\Omega}$. On subsection (2.7), calculations showed that both amplitude and phase modulations create coherent states on sidebands $(\delta=0)$, so there is no information loss when this term is not measured.

However a $\delta \neq 0$ term may exist if the state was not generated by classical modulations. This is the case for a squeezed state; or even a more complex state, exhibiting entanglement between sidebands. Measuring this state with usual homodyne detection yields the wrong result. Notwithstanding, recent publications show that this information can be retrieved by using electronic techniques [36].

\subsubsection{Self-homodyne detection}

When a cavity is used, the photocurrent operator is not proportional to eqn (2.50) anymore [16]. It needs to be changed according to the substitution in eqn (2.97).

$$
\hat{J}_{\Omega}(\Delta)=e^{-i \theta_{R}(\Delta)} r(\Delta) \hat{a}_{\Omega}+e^{i \theta_{R}(\Delta)} r^{*}(\Delta) \hat{a}_{-\Omega}^{\dagger}+e^{-i \theta_{R}(\Delta)} t(\Delta) \hat{b}_{\Omega}+e^{i \theta_{R}(\Delta)} t^{*}(\Delta) \hat{b}_{-\Omega}^{\dagger},
$$


showing that the phase acquired by the sidebands depends on the detuning and there is a mixture with vacuum fluctuations at $\Omega$ frequency.

Analogous treatment can be done to the new photocurrent and there will be again a sine and a cosine demodulated signals $\hat{J}_{\text {sin }}$ and $\hat{J}_{\text {cos }}$ (besides a vacuum term $\hat{J}_{\text {vac }}$ not written here).

$$
\begin{aligned}
& \hat{J}_{\cos }(\Delta)=x_{+} \hat{p}_{s}-y_{+} \hat{q}_{a}+x_{-} \hat{q}_{s}+y_{-} \hat{p}_{a}, \\
& \hat{J}_{\sin }(\Delta)=x_{+} \hat{q}_{a}+y_{+} \hat{p}_{s}-x_{-} \hat{p}_{a}+y_{-} \hat{q}_{s}
\end{aligned}
$$

where

$$
\begin{gathered}
x_{+}+i y_{+}=\frac{e^{-i \theta_{R}(\Delta)} r(\Delta)+e^{i \theta_{R}(\Delta)} r^{*}(\Delta)}{2}=g_{+}, \\
x_{-}+i y_{-}=\frac{i\left(e^{-i \theta_{R}(\Delta)} r(\Delta)-e^{i \theta_{R}(\Delta)} r^{*}(\Delta)\right)}{2}=g_{-} .
\end{gathered}
$$

The spectral noise power will then be

$$
S(\Omega)=\begin{gathered}
\left|g_{+}\right|^{2} \frac{\Delta^{2} \hat{p}_{s}+\Delta^{2} \hat{q}_{a}}{2}+\left|g_{-}\right|^{2} \frac{\Delta^{2} \hat{q}_{s}+\Delta^{2} \hat{p}_{a}}{2} \\
+\operatorname{Re}\left[g_{+}^{*} g_{-}\right]\left(C\left(\hat{p}_{s}, \hat{q}_{s}\right)-C\left(\hat{p}_{a}, \hat{q}_{a}\right)\right)+\operatorname{Im}\left[g_{+}^{*} g_{-}\right]\left(C\left(\hat{p}_{s}, \hat{p}_{a}\right)+C\left(\hat{q}_{s}, \hat{q}_{a}\right)\right),
\end{gathered}
$$

where $\operatorname{Re}[X]$ and $\operatorname{Im}[X]$ represents, respectively, the real and imaginary parts of $X$. Writing it in terms of the covariance matrix in eqn (2.105),

$$
S_{\Omega}(\Delta)=c_{\alpha} \alpha+c_{\beta} \beta+c_{\gamma} \gamma+c_{\delta} \delta
$$

This shows that every term can now be measured by using a cavity. The intuitive idea is that different frequencies get different phase shifts and attenuations by the cavity, enabling us to measure the differences on the lower and the upper sideband.

Figure (2.10) plots all parameters of eqn (2.119) as a function of $\Delta$ using the real measured parameters of the cavity of the experiment. The reflections $r_{1}$ and $r_{2}$ of eqn (2.94) are

$$
r_{1}=0.963, \quad r_{2}=0.999
$$

which leads to $d=|r(\Delta=0)|=0.948$. This value of $d$ close to 1 is the reason the measurement of the term $\delta$ is not possible.

The terms $c_{\alpha}$ and $c_{\beta}$ are related to the variances of quadratures $\hat{P}$ and $\hat{Q}$. As expected, the plot of $c_{\beta}$ equals 1 when the cavity is away from the resonant length for the beam (the measurement is performed on the $\hat{P}$ quadrature), while $c_{\alpha}$ equals 1 for $|\Delta|$ closer to zero (the measurement is performed on the $\hat{Q}$ quadrature).

The graphs for $c_{\alpha}$ and $c_{\beta}$ show they are even functions, in opposition to the odd functions $c_{\gamma}$ and $c_{\delta}$. Since these are the terms measuring asymmetries in the sidebands modes, this is the expected behaviour, we expect the functions to behave differently when the cavity is near the resonance of one of the sidebands or the other. The impossibility of measuring $\delta$ by our cavity is clear when the plot of $c_{\delta}$ is analysed, the peak of the function is 100 times smaller than the other graphs, resulting in a precision that is not enough to measure this term correctly.

\subsubsection{Measurement of two beams}

If modes of beams 1 and 2 are to be measured, the full state of the system is described by the four-mode covariance matrix

$$
\mathbf{V}_{s / a}^{(12)}=\left(\begin{array}{cc}
\mathbf{V}_{s}^{(12)} & \mathbf{C}_{s / a}^{(12)} \\
\left(\mathbf{C}_{s / a}^{(12)}\right)^{T} & \mathbf{V}_{a}^{(12)}
\end{array}\right)
$$



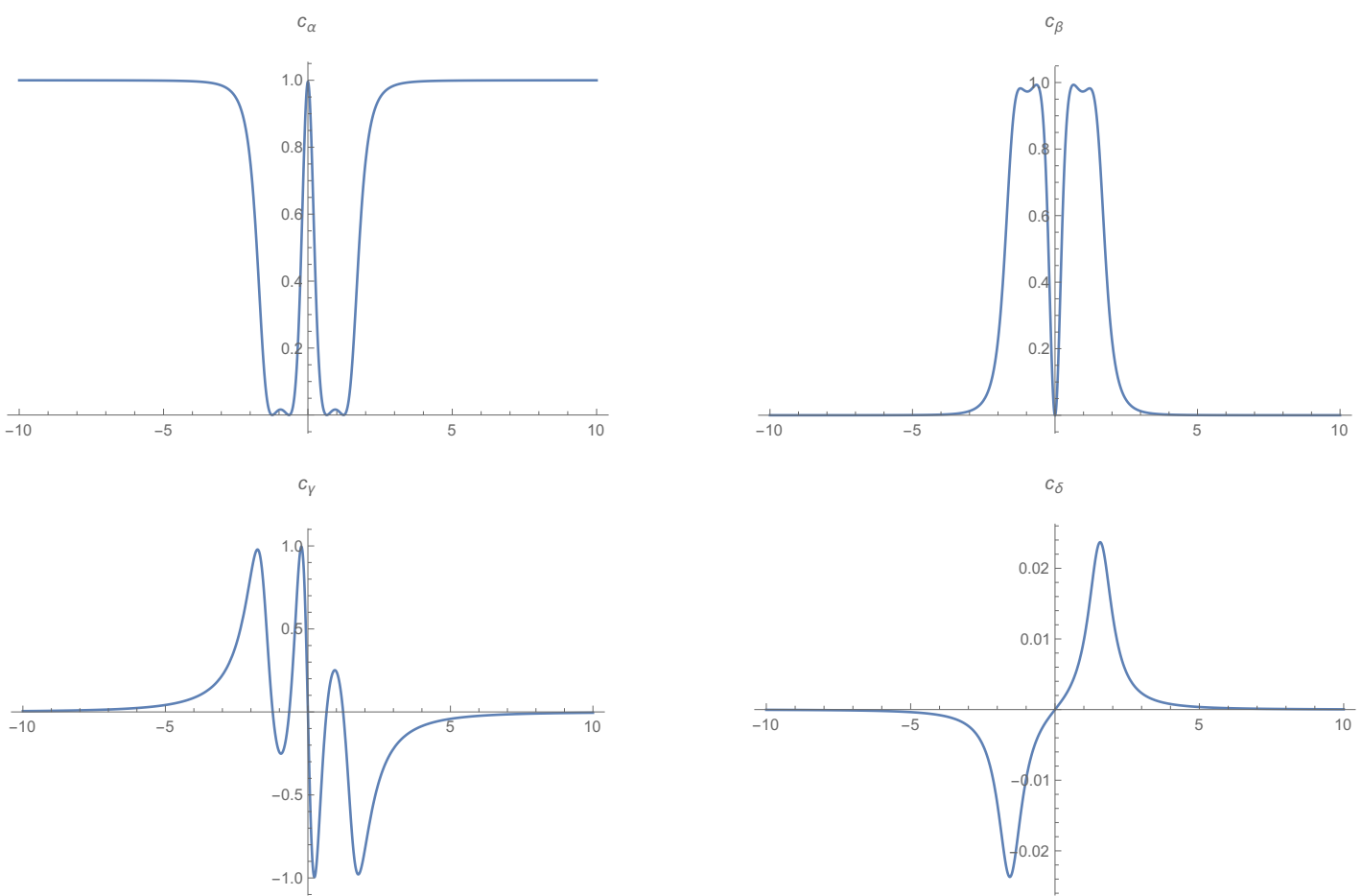

Figure 2.10: Parameters of eqn $(2.119)$ as a function of $\Delta$.

where the diagonal terms $\mathbf{V}_{s}^{(12)}$ and $\mathbf{V}_{a}^{(12)}$ are four by four matrices containing the variances and covariances of, respectively, both beams' symmetric and antisymmetric combination of quadratures. Stationarity implies that these two matrix must be the same up to a local basis rotation.

$$
\mathbf{V}_{s}^{(12)}=\left(\begin{array}{cccc}
\alpha^{(1)} & \gamma^{(1)} & \mu & \xi \\
\gamma^{(1)} & \beta^{(1)} & \zeta & \nu \\
\mu & \zeta & \alpha^{(2)} & \gamma^{(2)} \\
\xi & \nu & \gamma^{(2)} & \beta^{(2)}
\end{array}\right)
$$

where the new terms $\mu, \xi, \zeta$ and $\nu$ represents the correlations between quadratures of both beams.

On a single beam measurement, the off-diagonal element $\delta$ is invisible to standard homodyne detection. Here, all the terms on the off-diagonal matrix $\mathbf{C}_{s / a}^{(1 / 2)}$ requires a cavity to be accessed. Only then, the covariances between the $s$ and $a$ modes can be measured [16].

$$
\mathbf{C}_{s / a}^{(12)}=\left(\begin{array}{cccc}
\delta^{(1)} & 0 & \kappa & -\eta \\
0 & \delta^{(1)} & \tau & -\lambda \\
-\lambda & \eta & \delta^{(2)} & 0 \\
-\tau & \kappa & 0 & \delta^{(2)}
\end{array}\right)
$$

By constructing the matrix $\mathbf{V}_{s / a}^{(12)}$, it is possible to measure correlations between different beams and quantify the entaglement present on the system. This is the theme of next chapter. 


\section{ENTANGLEMENT GENERATION IN SECOND ORDER NON LINEAR MEDIA}

A famous event in the history of physics is the publication of the paper "Can QuantumMechanical Description of Physical Reality Be Considered Complete?" written by Einstein, Podolsky and Rosen [37]. It is the first time that an entangled state was discussed. Surprisingly, it is presented as an example showing that quantum mechanics doesn't describe physical reality completely. The discussed state (not normalized) on momentum space is

$$
\psi\left(q_{1}, q_{2}\right)=\int_{-\infty}^{\infty} e^{\frac{i 2 \pi}{\hbar}\left(q_{1}-q_{2}+q_{0}\right) p} d p
$$

representing a two-particle state where $q_{1}, q_{2}$ are the position coordinates for each particle, $p$ is the momentum and $q_{0}$ is a constant.

The eigenfunction of the position operator $\hat{q}_{1}$ for the first particle is

$$
u_{q}\left(q_{1}\right)=\delta\left(q_{1}-q\right)
$$

where $\delta(X)$ is the delta function for $(X)$. This corresponds the position operator to the eigenvalue $q$. By using this function, eqn (3.1) can be written as

$$
\psi\left(q_{1}, q_{2}\right)=\int_{-\infty}^{\infty} u_{q}\left(q_{1}\right) \varphi_{q}\left(q_{2}\right) d q,
$$

where we must have

$$
\varphi_{q}\left(q_{2}\right)=\int_{-\infty}^{\infty} e^{\frac{i 2 \pi}{h}\left(q-q_{2}+q_{0}\right)} d p=h \delta\left(q-q_{2}+q_{0}\right) .
$$

This is the eigenfunction of the position operator $\hat{q}_{2}$ for the second particle, corresponding to the eigenvalue $q+q_{0}$.

Similarly, we could calculate the eigenfunction for $\hat{p}_{1}=(h / 2 \pi i) \partial / \partial q_{1}$ and we would arrive at

$$
u_{p}\left(q_{1}\right)=e^{\frac{i 2 \pi}{h} q_{1} p}
$$

corresponding to the eigenvalue $p$. Analogously as done above for the position parameter, the second particle will be associated with the function

$$
\varphi_{p}\left(q_{2}\right)=e^{-\frac{i 2 \pi}{h}\left(q_{2}-q_{0}\right) p}
$$

which is the eigenfunction of the $\hat{p}_{2}$ operator associated with the eigenvalue $-p$.

We are lead to conclude that the measurement of one of the discussed parameters on one of the particles allows us to predict with certainty the measurement outcome of the second one. However, the quadratures $q$ and $p$ for each particle are represented by non-commuting observables, which means that acquiring knowledge of one of the quadratures precludes the knowledge of the other. In the words of the authors, the quadrature chosen to be measured is an element of reality while the other is not. Nonetheless, depending on the quadrature chosen to be measured on one particle, we are lead to a different wavefunction describing the same reality for the second particle. This is the basis of the so called EPR paradox.

This phenomenon was only understood 30 years later [38], after which the manipulations of the so called entangled states became an interesting subject of research. The first studies were on the discrete variables regime, where the outcomes of measurements can only assume some discrete values, different from the state of eqn (3.1) which exhibits correlations on variables that can assume continuous values.

By the end of the 1980's, it was shown that some systems that were used for the study of nonlinear optics could also show correlations mathematically equivalent as envisioned by the EPR paper [39]. These correlations could be studied in the amplitude and phase quadratures of light beams by using a 
system called optical parametric oscillator (OPO), described on section (3.1). The output of such a device is a two-mode squeezed state, where the sum of two states' phase quadratures and the difference of their amplitudes will be squeezed (noise reduced) while the other combination of quadratures - difference of phases and sum of amplitudes - will be anti-squeezed (noise enhanced).

$$
\begin{aligned}
\Delta^{2} \hat{P}_{+} & =e^{2 r}, & \Delta^{2} \hat{P}_{-}=e^{-2 s}, \\
\Delta^{2} \hat{Q}_{+} & =e^{-2 r}, & \Delta^{2} \hat{Q}_{-}=e^{2 s},
\end{aligned}
$$

resembling eqns (2.26) for the one-mode squeezing.

When the squeezing parameters $r$ and $s$ are infinite, the correlations exhibited on the beams generated by the OPO are the same as on the original paper by Einstein. The knowledge of one of the quadratures of one beam allows us to infer with certainty the value of this quadrature for the second beam. This is the case of maximal entanglement. For more realistic cases, we have finite squeezing parameters, allowing us to infer with a small uncertainty the quadrature value on a second beam after a measurement performed on the first.

Section (3.2) deals with the experimental implementation of the discussed on section (3.1). Its subsections will explain the details of the construction of the utilized OPO as well as the auxiliary systems that make it work, such as the filter cavity (subsection (3.2.1)) and the chamber where the crystal is placed (subsection (3.2.2)).

\section{$3.1 \quad$ OPO}

Roughly, an OPO is a nonlinear gain medium inside an optical cavity. During this thesis, the only type of OPO that will be discussed is the one composed by a KTP second order non linear crystal is used as the gain medium [14].

\subsubsection{Non linear optics}

First, a classical description of the process will be given. The electromagnetic field will be written as

$$
\mathbf{E}(\mathbf{x}, t)=\alpha e^{i(\mathbf{k} \cdot \mathbf{x}-\omega t)} \epsilon+\text { c.c. },
$$

where c.c. stands for complex conjugate and $\mathbf{k}$ is the wave vector.

Usually, an interaction between a classical electromagnetic field with matter [17] results in atoms on the material linearly polarized by the field

$$
\mathbf{P}(\mathbf{x}, t)=\epsilon_{0} \chi^{(1)} \mathbf{E}^{(+)}(\mathbf{x}, t)
$$

where $\chi^{(1)}$ is a number representing the linear susceptibility of the material. As a result, a wave can be propagated if it satisfies Maxwell's equation

$$
\nabla^{2} \mathbf{E}-\frac{1}{c^{2}} \frac{\partial^{2}}{\partial t^{2}}\left(\mathbf{E}+\frac{\mathbf{P}}{\epsilon_{0}}\right)=0
$$

Non linear optics studies a more general type of interaction, where the polarizability may be written as

$$
P_{i}(\mathbf{x}, t)=\epsilon_{0} \sum_{j} \chi_{i j}^{(1)} E_{j}(\mathbf{x}, t)+\epsilon_{0} \sum_{j, k} \chi_{i j k}^{(2)} E_{j}(\mathbf{x}, t) E_{k}(\mathbf{x}, t)+\epsilon_{0} \sum_{j, k, l} \chi_{i j k l}^{(3)} E_{j}(\mathbf{x}, t) E_{k}(\mathbf{x}, t) E_{l}(\mathbf{x}, t)+\ldots
$$

On the above equation, $P_{i}$ represents the component of $\mathbf{P}$ on the $i$ axis. The linear order susceptibility is no longer a number, but a tensor of rank 2 , with dependence on both the polarizalibity coordinates 
as the electric field coordinates. The second order term is a rank 3 tensor, and it is the most important term for the work described on this thesis, since all other $\chi^{(n)}$ are approximately zero.

Exploring this term, we can consider the interaction of two waves in the material

$$
\begin{aligned}
& \mathbf{E}_{1}(\mathbf{x}, t)=\alpha_{1} e^{i\left(\mathbf{k}_{1} \cdot \mathbf{x}-\omega_{1} t\right)} \epsilon_{1}+c . c . \\
& \mathbf{E}_{2}(\mathbf{x}, t)=\alpha_{2} e^{i\left(\mathbf{k}_{2} \cdot \mathbf{x}-\omega_{2} t\right)} \epsilon_{2}+c . c .
\end{aligned}
$$

giving rise to a total electric field $\mathbf{E}(\mathbf{x}, t)=\mathbf{E}_{1}(\mathbf{x}, t)+\mathbf{E}_{2}(\mathbf{x}, t)$. Considering $\chi^{(2)}$ equal for each term, the polarizability on this order may be written as

$$
\begin{array}{r}
P^{(2)}=\epsilon \chi^{(2)} E^{2}(\mathbf{x}, t)=\epsilon_{0} \chi^{(2)}\left[\alpha_{1}^{2} e^{i\left(2 \mathbf{k}_{1} \mathbf{x}-2 \omega_{1} t\right)}+\text { c.c. }+\alpha_{2}^{2} e^{i\left(2 \mathbf{k}_{2} \mathbf{x}-2 \omega_{2} t\right)}+2\left(\left|\alpha_{1}\right|^{2}+\left|\alpha_{2}\right|^{2}\right)+\right. \\
\left.+2 \alpha_{1} \alpha_{2} e^{i\left(\left(\mathbf{k}_{1}+\mathbf{k}_{2}\right) \mathbf{x}-\left(\omega_{1}+\omega_{2}\right) t\right)}+\text { c.c. }+2 \alpha_{1} \alpha_{2}^{*} e^{i\left(\left(\mathbf{k}_{1}-\mathbf{k}_{2}\right) \mathbf{x}-\left(\omega_{1}-\omega_{2}\right) t\right)}+\text { c.c. }\right],
\end{array}
$$

showing four optical effects [20]:

- second harmonic generation - on the terms $\alpha_{1}^{2} e^{i\left(2 \mathbf{k}_{1} \mathbf{x}-2 \omega_{1} t\right)}+$ c.c. $+\alpha_{2}^{2} e^{i\left(2 \mathbf{k}_{2} \mathbf{x}-2 \omega_{2} t\right)}$,

- optical retification - terms $2\left(\left|\alpha_{1}\right|^{2}+\left|\alpha_{2}\right|^{2}\right)$,

- frequency addition $-2 \alpha_{1} \alpha_{2} e^{i\left(\left(\mathbf{k}_{1}+\mathbf{k}_{2}\right) \mathbf{x}-\left(\omega_{1}+\omega_{2}\right) t\right)}+$ c.c,

- and frequency difference $-2 \alpha_{1} \alpha_{2}^{*} e^{i\left(\left(\mathbf{k}_{1}-\mathbf{k}_{2}\right) \mathbf{x}-\left(\omega_{1}-\omega_{2}\right) t\right)}+$ c.c..

This work explores the first and third of these effects, explained below.

Second harmonic generation receives an input of frequency $\omega$ and generate a wave of frequency $2 \omega$. On the experiment here described, the laser used is a Diabolo, made by Innolight, with two output fields. One is a $1064 \mathrm{~nm}$ infrared laser (also called $I R$ ), the other is $532 \mathrm{~nm}$ green light generated by the effect in question using the $I R$ field. This makes possible that two different frequencies of light can be used on the experiment.

Frequency addition is also called parametric down conversion. It is an effect happening on the OPO. The laser's green light (frequency $\omega_{0}$ ) is directed to a cavity with a second order crystal inside, the output of this cavity will be two different beams on the infrared spectrum (frequencies $\omega_{1}$ and $\omega_{2}$ ) with the property $\omega_{1}+\omega_{2}=\omega_{0}$. This effect is also known as three wave mixing. This is the opposite effect of second harmonic generation, where there were two photons generating one, now there is only one photon that generates two.

\subsubsection{Quantum description}

Two conditions must be met in order for the discussed processes to work. One of them is energy conservation (fig (3.1))

$$
\hbar \omega_{0}=\hbar \omega_{1}+\hbar \omega_{2}
$$

The other condition is the conservation of the photons' momenta, satisfying a condition called phase matching

$$
\hbar \mathbf{k}_{0}=\hbar \mathbf{k}_{1}+\hbar \mathbf{k}_{2}
$$

The Hamiltonian for this process is [31]

$$
\hat{H}_{i n t}=i \hbar \frac{2 \chi(2)}{\tau}\left(\hat{a}_{0}^{\dagger} \hat{a}_{1} \hat{a}_{2}-\hat{a}_{0} \hat{a}_{1}^{\dagger} \hat{a}_{2}^{\dagger}\right)
$$

where $\tau$ is related to the interaction time with the non-linear medium. This Hamiltonian describes the destruction of one photon (called pump, mode indicated by indice 0) and the creation of two others of 
well defined frequency modes (the photon pair called twin beams, being one of them signal, mode 1 and the other idler, mode 2), and also the opposite process. This is schematically shown on fig (3.1).

For the full Hamiltonian, one needs to add the free field Hamiltonians for each mode $i$ (eqn (2.8)

$$
\hat{H}_{i}=\hbar \omega_{i}\left(\hat{a}_{i}^{\dagger} \hat{a}_{i}+\frac{1}{2}\right)
$$

It is possible to show that the Hamiltonian commutes with $2 \hat{n}_{0}-\hat{n}_{1}-\hat{n}_{2}$, where $\hat{n}_{i}=\hat{a}_{i}^{\dagger} \hat{a}_{i}$ is the operator for the number of photons. The satisfaction of this commutation means that the destruction of one pump photon gives rise to the creation of the twin beams, almost instantaneously [20]. On the number basis, an initial state $\left|n_{0}, n_{1}, n_{2}\right\rangle$ will lead to a final state $\left|n_{0}-1, n_{1}+1, n_{2}+2\right\rangle$.

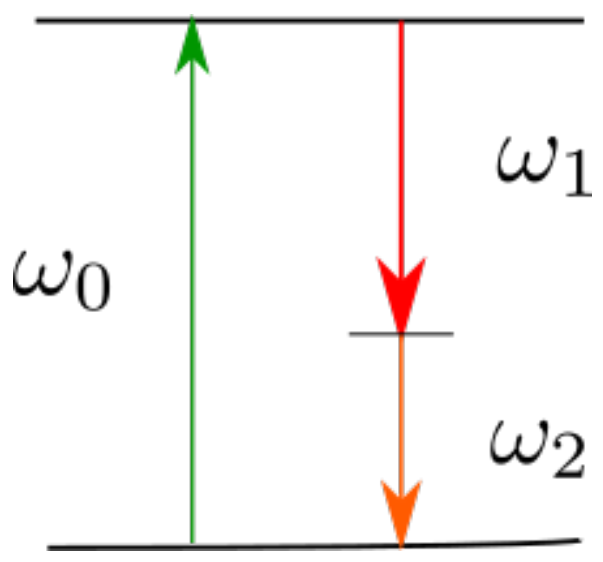

Figure 3.1: Pictorial description of parametric down conversion showing conservation of energy. Photon with frequency $\omega_{0}$ is annihilated and photons with frequencies $\omega_{1}$ and $\omega_{2}$ are created.

The output state of this process is called two-mode squeezed vacuum. Pairs of photons are generated from time to time, but not bright beams. To suit our needs, the crystal was put inside a cavity that resonates for the three fields involved. It can then generate the desired bright beams while also lowering the power of the laser we need to use for the process to occur. It adds extra terms to the Hamiltonian. One of them should represent the strong classical pump entering the cavity

$$
\hat{H}_{i n}=i \hbar \frac{\sqrt{2 t_{0}^{1}}}{\tau} \alpha_{0}^{i n}\left(\hat{a}_{0}-\hat{a}_{0}^{\dagger}\right)
$$

where $\alpha_{0}^{i n}$ is the input field's amplitude and $t_{0}^{1}$ is the transmissivity of the first mirror for this frequency. The free field Hamiltonians must be replaced by the cavity Hamiltonians for each mode $i$

$$
\hat{H}_{i}=\hbar \frac{2 g_{i}}{\tau} \Delta_{i} \hat{a}_{i}^{\dagger} \hat{a}_{i}
$$

where $\Delta_{i}=\frac{\omega_{i}-\omega_{i R}}{\gamma_{i}}$ is the detuning for each mode normalized by the bandwidth, and $g_{i}$ is the mode dependent loss term. Considering $\omega_{1}$ and $\omega_{2}$ close to each other (as it is on this experiment), it can be considered that $\Delta_{1}=\Delta_{2}=\Delta$ and $g_{1}=g_{2}=g$.

The full Hamiltonian is the sum of each of the above

$$
\hat{H}=\sum_{i} \hat{H}_{i}+\hat{H}_{i n}+\hat{H}_{i n t}
$$

Solving the above Hamiltonian for the dynamics of creation and annihilation operators is hard. It relies on open quantum systems formalism and the use of Wigner functions. Here, the final solutions will be presented and comments and properties of them will be discussed. For the full calculation, we 
recommend some thesis of the group [34, 31]. The solution for each $\hat{a}_{i}=\alpha_{i}+\delta \hat{a}_{i}=\left|\alpha_{i}\right| e^{i \theta_{i}}$ is

$$
\begin{aligned}
\tau \frac{d \hat{a}_{0}}{d t} & =-g_{0}\left(1-i \Delta_{0}\right) \hat{a}_{0}-2 \chi^{(2)} \hat{a}_{1} \hat{a}_{2}+\hat{a}_{0}^{i n} \sqrt{2 t_{0}^{1}}+\delta \hat{v}_{0} \sqrt{2 t_{0}^{2}}, \\
\tau \frac{d \hat{a}_{1}}{d t} & =-g(1-i \Delta) \hat{a}_{1}+2 \chi^{(2)} \hat{a}_{0} \hat{a}_{2}^{\dagger}+\delta \hat{u}_{1} \sqrt{2 t_{1}^{1}}+\delta \hat{v}_{1} \sqrt{2 t_{1}^{2}} \\
\tau \frac{d \hat{a}_{2}}{d t} & =-g(1-i \Delta) \hat{a}_{2}+2 \chi^{(2)} \hat{a}_{0} \hat{a}_{1}^{\dagger}+\delta \hat{u}_{2} \sqrt{2 t_{2}^{1}}+\delta \hat{v}_{2} \sqrt{2 t_{2}^{2}}
\end{aligned}
$$

where $t_{i}^{n}$ is the transmissivity of the $n^{\prime}$ th mirror for mode $i$ and $\delta \hat{v}_{i}, \delta \hat{u}_{i}$ are vacuum fluctuations on this mode.

By taking the average of the above equations, one can neglect the quantum fluctuations terms and arrive at solutions for the mean values $\alpha_{i}$. Writing it in polar coordinates, one obtains

$$
\begin{array}{r}
g_{0}(1-i \Delta)\left|\alpha_{0}\right| e^{i \theta_{0}}+2 \chi^{(2)}|\alpha|^{2} e^{i \theta_{+}}-\alpha_{0}^{i n} \sqrt{2 t_{0}^{1}}=0, \\
g(1-i \Delta)-2 \chi^{(2)}\left|\alpha_{0}\right| e^{i\left(\theta_{0}-\theta_{+}\right)}=0,
\end{array}
$$

where $\left|\alpha_{1}\right|=\left|\alpha_{2}\right|=|\alpha|$ and $\theta_{+}=\theta_{1}+\theta_{2}$.

Some mathematical manipulations can yield

$$
e^{i \theta_{0}}=\frac{1-i \Delta}{\sqrt{1+\Delta^{2}}} e^{i \theta_{+}}
$$

providing a relation between the phases of the twin beams and the pump.

Going further, these equations can also yield

$$
|\alpha|^{2}=\sqrt{\frac{2 t_{0}^{1}\left(\alpha_{0}^{i n}\right)^{2}}{4\left(\chi^{(2)}\right)^{2}}-\left[\frac{g_{0} g\left(\Delta_{0}+\Delta\right)}{4\left(\chi^{(2)}\right)^{2}}\right]^{2}}-\frac{g_{0} g\left(1-\Delta_{0} \Delta\right)}{4\left(\chi^{(2)}\right)^{2}},
$$

the power of the twin beams generated $|\alpha|^{2}$ as a function of the input power $\left(\alpha_{0}^{i n}\right)^{2}$. From this equation one can calculate the minimal input power for which twin beams may be generated. By setting $|\alpha|^{2}=0$, one obtains

$$
\left(\alpha_{0}^{i n}\right)_{\min }^{2}=\frac{g_{0}^{2} g^{2}}{8 t_{0}^{1}\left(\chi^{(2)}\right)^{2}}
$$

this is called threshold power and it can easily be measured in the laboratory by slowly increasing the pump power until twin beams are generated. This measurement can be done everyday and compared to check if the losses $g_{0}^{2} g^{2}$ have increased or not.

After finding the solutions for the mean values of the generated fields written above, we can now find the solutions for the fluctuations. The equations to be solved are

$$
\begin{gathered}
\tau \frac{d}{d t} \delta \hat{a}_{0}=-g\left(1-i \Delta_{0}\right) \delta \hat{a}_{0}-2 \chi^{(2)}|\alpha|\left(e^{i \theta_{2}} \delta \hat{a}_{1}+e^{i \theta_{1}} \delta \hat{a}_{2}\right)+\delta \hat{a}_{0}^{i n} \sqrt{2 t_{0}^{1}}+\delta \hat{v}_{0} \sqrt{2 t_{0}^{2}} \\
\tau \frac{d}{d t} \delta \hat{a}_{1}=-g(1-i \Delta) \delta \hat{a}_{1}+2 \chi^{(2)}\left(|\alpha| e^{-i \theta_{2}} \delta \hat{a}_{0}+\left|\alpha_{0}\right| e^{i \theta_{0}} \delta \hat{a}_{2}^{\dagger}\right)+\delta \hat{u}_{1} \sqrt{2 t_{1}^{1}}+\delta \hat{v}_{1} \sqrt{2 t_{1}^{2}} \\
\tau \frac{d}{d t} \delta \hat{a}_{2}=-g(1-i \Delta) \delta \hat{a}_{2}+2 \chi^{(2)}\left(|\alpha| e^{-i \theta_{1}} \delta \hat{a}_{0}+\left|\alpha_{0}\right| e^{i \theta_{0}} \delta \hat{a}_{1}^{*}\right)+\delta \hat{u}_{2} \sqrt{2 t_{2}^{1}}+\delta \hat{v}_{2} \sqrt{2 t_{2}^{2}}
\end{gathered}
$$

It is convenient to separate the quadratures of the fluctuations, including the phase of the state in the description

$$
\begin{gathered}
\delta \hat{a}_{i}=\frac{e^{i \theta_{i}}}{2}\left(\delta \hat{P}_{i}+i \hat{Q}_{i}\right), \\
\delta \hat{u}_{i}=\frac{e^{i \theta_{i}}}{2}\left(\delta \hat{P}_{u i}+i \hat{Q}_{u i}\right), \\
\delta \hat{v}_{i}=\frac{e^{i \theta_{i}}}{2}\left(\delta \hat{P}_{v i}+i \hat{Q}_{v i}\right) .
\end{gathered}
$$


The quadrature operators can be divided in the vectors

$$
\begin{aligned}
\delta \widehat{\mathbf{a}} & =\left(\begin{array}{llllll}
\delta \hat{P}_{0} & \delta \hat{Q}_{0} & \delta \hat{P}_{1} & \delta \hat{Q}_{1} & \delta \hat{P}_{2} & \left.\delta \hat{Q}_{2}\right)^{T}, \\
\delta \widehat{\mathbf{u}} & =\left(\begin{array}{llllll}
\delta \hat{P}_{i n} & \delta \hat{Q}_{i n} & \delta \hat{P}_{u 1} & \delta \hat{Q}_{u 1} & \delta \hat{P}_{u 2} & \delta \hat{Q}_{u 2}
\end{array}\right)^{T}, \\
\delta \widehat{\mathbf{v}} & =
\end{array} \begin{array}{llllll}
\delta \hat{P}_{v 0} & \delta \hat{Q}_{v 0} & \delta \hat{P}_{v 1} & \delta \hat{Q}_{v 1} & \delta \hat{P}_{v 2} & \left.\delta \hat{Q}_{v 2}\right)^{T}
\end{array}\right.
\end{aligned}
$$

After these transformations, one can arrive at the equation

$$
\frac{d}{d t} \delta \widehat{\mathbf{a}}=M \delta \widehat{\mathbf{a}}+T_{1} \delta \widehat{\mathbf{u}}+T_{2} \delta \widehat{\mathbf{v}}
$$

where $T_{1}=\operatorname{diag}\left(\sqrt{2 t_{0}^{1}}, \sqrt{2 t_{0}^{1}}, \sqrt{2 t_{1}^{1}}, \sqrt{2 t_{1}^{1}}, \sqrt{2 t_{2}^{1}}, \sqrt{2 t_{2}^{1}}\right)$ and $T_{2}=\operatorname{diag}\left(\sqrt{2 t_{0}^{2}}, \sqrt{2 t_{0}^{2}}, \sqrt{2 t_{1}^{2}}, \sqrt{2 t_{1}^{2}}, \sqrt{2 t_{2}^{2}}, \sqrt{2 t_{2}^{2}}\right)$ are diagonal matrices containing the terms due to losses and

$$
M=\left(\begin{array}{cccccc}
-g_{0} & g_{0} \Delta_{0} & -\eta & \Delta \eta & -\eta & \Delta \eta \\
g_{0} \Delta_{0} & -g_{0} & -\Delta \eta & -\eta & -\Delta \eta & -\eta \\
\eta & \Delta \eta & -g & g \Delta & g & -g \Delta \\
-\Delta \eta & \eta & -g \Delta & -g & -g \Delta & -g \\
\eta & \Delta \eta & g & -g \Delta & -g & g \Delta \\
-\Delta \eta & \eta & -g \Delta & -g & -g \Delta & -g
\end{array}\right),
$$

where $\eta=\frac{2 \chi^{(2)}|\alpha|}{\sqrt{1+\Delta^{2}}}$.

By Fourier transform, we can go to the frequency space, writing eqn (3.40) as

$$
\delta \widehat{\mathbf{a}}(\Omega)=-\frac{1}{M+2 i g \Omega I}\left(T_{1} \delta \widehat{\mathbf{u}}(\Omega)+T_{2} \delta \widehat{\mathbf{v}}\right),
$$

describring the resulting quadratures inside the cavity. To get the output quadratures, one needs to apply $T_{2}$ to the above (because of the extra transmission) and consider the reflection of the vacuum state on the output mirror of the OPO cavity

$$
\begin{aligned}
\delta \widehat{\mathbf{a}}_{\text {out }}(\Omega) & =T_{1} \delta \widehat{\mathbf{a}}(\Omega)-\delta \widehat{\mathbf{v}}(\Omega), \\
& =-T_{1} \frac{1}{M+2 i g \Omega} T_{1} \delta \widehat{\mathbf{u}}(\Omega)-\left(T_{1} \frac{1}{M+2 i g \Omega} T_{2}+I\right) \delta \widehat{\mathbf{v}}(\Omega) .
\end{aligned}
$$

With the obtained information, it is possible to construct the covariance matrix of this system.

\subsubsection{Sum and subtraction space}

The sum and subtraction of the twin beams' quadratures are given by

$$
\begin{aligned}
\delta \hat{P}_{ \pm} & =\frac{\delta \hat{P}_{1} \pm \delta \hat{P}_{2}}{\sqrt{2}}, \\
\delta \hat{Q}_{ \pm} & =\frac{\delta \hat{Q}_{1} \pm \delta \hat{Q}_{2}}{\sqrt{2}} .
\end{aligned}
$$

The noise spectrum is defined by (eqn (2.104))

$$
S_{\hat{x}}(\Omega)=\langle\delta \hat{x}(\Omega) \delta \hat{x}(-\Omega)\rangle .
$$

For the sum and subtraction of quadratures, the result is

$$
\begin{array}{lc}
S_{\hat{P}_{-}}= & 1-\frac{t}{g} \frac{1}{1+\nu^{2}}, \\
S_{\hat{Q}_{-}}= & 1+\frac{t}{g} \frac{1}{\nu^{2}}, \\
S_{\hat{P}_{+}}= & \left|\kappa_{p}\right|^{2} S_{p_{0}}^{i n}+|2 t \xi-1|^{2}+\frac{g_{0}-t_{0}}{t_{0}}\left|\kappa_{p}\right|^{2}+4 t(g-t)|\xi|^{2}, \\
S_{\hat{Q}_{+}}= & \left|\kappa_{q}\right|^{2} S_{q_{0}}^{\text {in }}+|2 t \zeta-1|^{2}+\frac{g_{0}-t_{0}}{t_{0}}\left|\kappa_{q}\right|^{2}+4 t(g-t)|\zeta|^{2},
\end{array}
$$


where

$$
\begin{array}{cc}
\kappa_{p}= & \frac{2 \sqrt{2} g|\alpha| \sqrt{t_{0} t} \xi}{\left|\alpha_{0}\right|\left(g_{0}+2 i g \nu\right)}, \\
\kappa_{q}= & \frac{2 \sqrt{2} g|\alpha| \sqrt{t_{0} t}}{\left|\alpha_{0}\right| \zeta\left(g_{0}+2 i g \nu\right)}, \\
\xi= & 2 i g \nu+\frac{2 g^{2}|\alpha|^{2}}{\left|\alpha_{0}\right|^{2}+2 i g \nu}, \\
\zeta= & \left(2 g+2 i g \nu+\frac{2 g^{2}|\alpha|^{2}}{\left|\alpha_{0}\right|^{2}\left(g_{0}+2 i g \nu\right)}\right)^{-1},
\end{array}
$$

and $\nu=\Omega / \gamma$, the frequency $\Omega$ divided by the OPO's bandwidth. Even though the expressions above don't make it intuitive at the first look, the noise quadratures $\delta \hat{P}_{-}$and $\delta \hat{Q}_{+}$are squeezed and the noise quadratures $\delta \hat{P}_{+}$and $\delta \hat{Q}_{-}$are anti-squeezed.

Experimentally, the degree of entanglement (or squeezing) can be measured by comparison with the Duan criteria [40]. Consider the sum of the two variances

$$
\Delta^{2} \hat{P}_{-}+\Delta^{2} \hat{Q}_{+}=\frac{1}{2}\left(\Delta^{2} \hat{P}_{1}+\Delta^{2} \hat{P}_{2}+\Delta^{2} \hat{Q}_{1}+\Delta^{2} \hat{Q}_{2}\right)-C\left(\hat{P}_{1}, \hat{P}_{2}\right)-C\left(\hat{Q}_{1}, \hat{Q}_{2}\right) .
$$

If there is no relation whatsoever between both beams, the correlation terms are zero.

$$
\Delta^{2} \hat{P}_{-}+\Delta^{2} \hat{Q}_{+}=\frac{1}{2}\left(\Delta^{2} \hat{P}_{1}+\Delta^{2} \hat{P}_{2}+\Delta^{2} \hat{Q}_{1}+\Delta^{2} \hat{Q}_{2}\right) .
$$

The least value for each term on the right hand side will then be 1 , shot noise, leading to

$$
\Delta^{2} \hat{P}_{-}+\Delta^{2} \hat{Q}_{+} \geq 2
$$

This is Duan's inequality. If the measured number for this quantity is smaller than 2 then the states are entangled.

\subsubsection{Measurement}

The bandwitdh of the OPO's cavity sets a maximum frenquency for which entanglement is present [31]. The lower the frequency chosen for the work, farther from the classical limit Duan criteria will be. For this reason, it is interesting to build a cavity with a high bandwidth and perform measurements on a low frequency.

The power of light is another limiting factor on the available squeezing. Although it is much easier to work with high energies - it is experimentally more stable since a shake in energy will not lead to a power lower than threshold power - it is with lower energies that entanglement presents itself greatly, in the sense that Duan criteria yields better results. Therefore, it is more convenient to work closer to threshold, even though it is experimentally harder.

In order to measure the correlations, this work makes use resonator detection technique to build the four-mode covariance matrix of the system, defined on eqn (2.121). One of the beams is separated in two by means of a beam-splitter, and each end is used for a different quadrature measurement, either $\hat{P}$ or $\hat{Q}$. The former is measured by simply sending light to the detectors, while the latter must first be sent to a cavity. As shown on subsection (2.8.2), the resulting demodulated signals will be

$$
\begin{gathered}
\hat{P}_{\cos }=\frac{\hat{p}_{s}}{\sqrt{2}}, \quad \hat{P}_{\sin }=\frac{\hat{q}_{a}}{\sqrt{2}}, \\
\hat{Q}_{\cos }=\frac{\hat{p}_{a}}{\sqrt{2} \cos \alpha+\frac{\hat{q}_{s}}{\sqrt{2}} \sin \alpha,} \quad \hat{Q}_{\sin }=-\frac{\hat{p}_{a}}{\sqrt{2}} \sin \alpha+\frac{\hat{q}_{s}}{\sqrt{2} \cos \alpha,}
\end{gathered}
$$

where the notation $\hat{X}_{y}$ represents the $y$ modulation for the photocurrent associated with the $\hat{X}$ quadrature. By measuring the variances and covariances of all these signals, all terms of the single-mode covariance matrix (2.105) can be calculated. 
The properties of the other beam are measured by the output of a scanning optical cavity. The desired terms can be retrieved by the fitting of the demodulated equations exhibited on subsection (2.9.2). The only one we don't have access is $\delta$, as explained on section (2.9). The reasons for applying different strategies for measuring each beam will become clearer on chapter 1 .

As will be shown on subsection (3.2.4), it is interesting to apply some rotations on the phase space to the measurement of correlations.

\subsection{Experimental methods}

On this section, experimental details of the so called EPR station will be given, this is where the OPO is located and entanglement is produced. For the effective production of entangled states, it is important to first reduce the phase excess noise on the pump laser. A filter cavity is used to achieve this goal, as will be explained on subsection (3.2.1). The experimental properties of the OPO used are given in the next subsections, along with auxiliary system of the setup.

\subsubsection{Filter cavity}

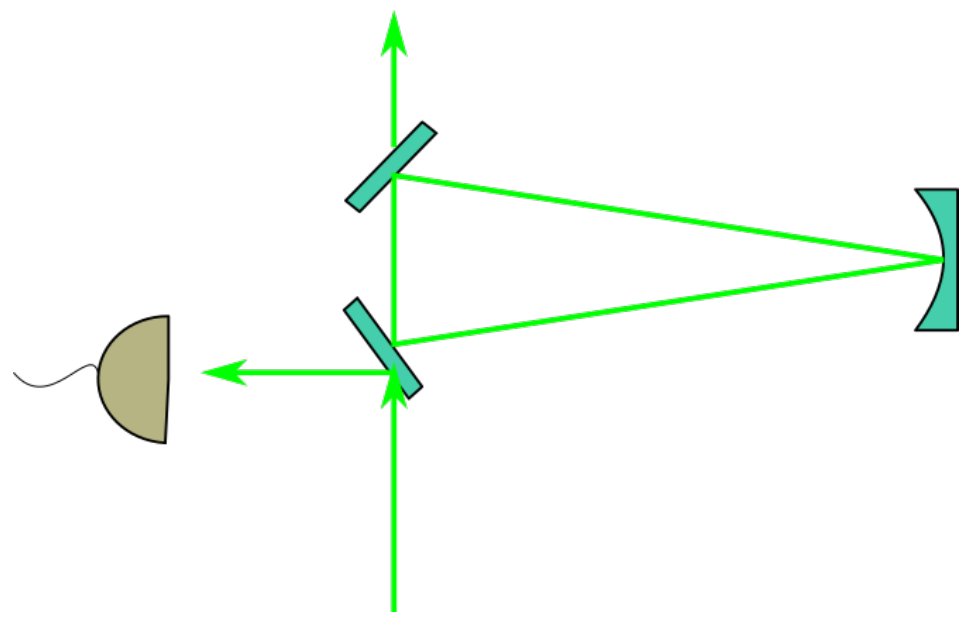

Figure 3.2: Drawing of filter cavity. Light comes from below, part of it is transmitted by the mirror and goes inside the cavity and another part is reflected and sent to the locking system. The transmission of the cavity is the upper part, which is directed to the rest of the system.

Our pump presents excess noise in phase, i.e., its noise is greater than the standard quantum noise level, harming the phase squeezing of the produced twin beams. To avoid this problem, the undesired noise can be reduced by the use of an optical cavity, called filter cavity when used for this purpose, schematically represented on fig (3.2). On this experiment, it was built with three mirrors, being one of them highly reflective and with a radius of curvature of $1 \mathrm{~m}$. The remaining mirrors are both $1 \%$ transmission and are the biggest responsible for the losses inside the cavity. The finesse is given by $F=\frac{\pi \sqrt{r_{1} r_{2}}}{1-r_{1} r_{2}}$, where indices 1 and 2 represent the lossy input and output mirrors.

Eqns (2.94) and (2.95) are used to derive the cavity's parameters. Its bandwidth is $700 \mathrm{kHz}$ and for the analisys frequency of $15 \mathrm{MHz}$, chosen for the production of sidebands, the attenuation is around $-75 \mathrm{~dB}$. The cavity is $113 \mathrm{~cm}$ long. It yields a waist for the beam of around $290 \mathrm{~mm}$. The finesse is 314 and the free spectral range is $265 \mathrm{M} \mathrm{Hz}$. It is locked at peak transmission by means of an electronic technique called Pound-Drever-Hall locking (appendix (A .3)). 


\subsubsection{OPO}

The crystal used is made of potassium titanyl phosphate (it is a KTP crystal) ordered from Raicol company and made for a $532 \mathrm{~nm}$ pump laser generate two fields around $1064 \mathrm{~nm}$ (one of them is $1055 \mathrm{~nm}$ while the other is $1072 \mathrm{~nm}$, this measurement is explained on appendix (B)). It is cut for type II phase matching, which means that the generated beams have orthogonal polarizations. It is 12 $\mathrm{mm}$ long and placed inside a $19 \mathrm{~mm}$ long linear cavity with plane-concave mirrors of 50 mm radius of curvature. Their transmission for each wavelength are in table (3.1), where $T_{i}^{\lambda}$ is the transmission of the $i$ 'th mirror in the cavity for the wavelength $\lambda$.

$$
\left|\begin{array}{c|c}
T_{1}^{532}=30 \% & T_{1}^{1064}=0.01 \% \\
T_{2}^{532}=0.01 \% & T_{2}^{1064}=4 \%
\end{array}\right|
$$

Table 3.1: Transmission of each OPO mirror.

Mirror 1, the input mirror, is highly reflective for $I R$ in order to avoid leaking from this port. The output of the OPO must be captured from the second mirror, which is more transmissive for this wavelength. We don't want leaking from the second port for the pump, supposed to leave the cavity from the input mirror, where a system for capturing the reflected beam is located in order to use the signal for cavity locking, as depicted on fig (3.3).

The waist of the beam inside the cavity is $28 \mu \mathrm{m}$ for green and $39 \mu \mathrm{m}$ for $I R$. The free spectral range is $5.2 \mathrm{GHz}$ and the bandwidth is $160 \mathrm{MHz}$ for green and $55 \mathrm{MHz}$ for $\mathrm{IR}$. Finesse is 100 for $I R$ and for green this value can lay between 20 and 30, depending on atmospheric conditions, explained on subsection (3.2.3). The cavity is designed so all three fields can resonate at the same time. The locking method selected is dither locking (appendix (A .2)).

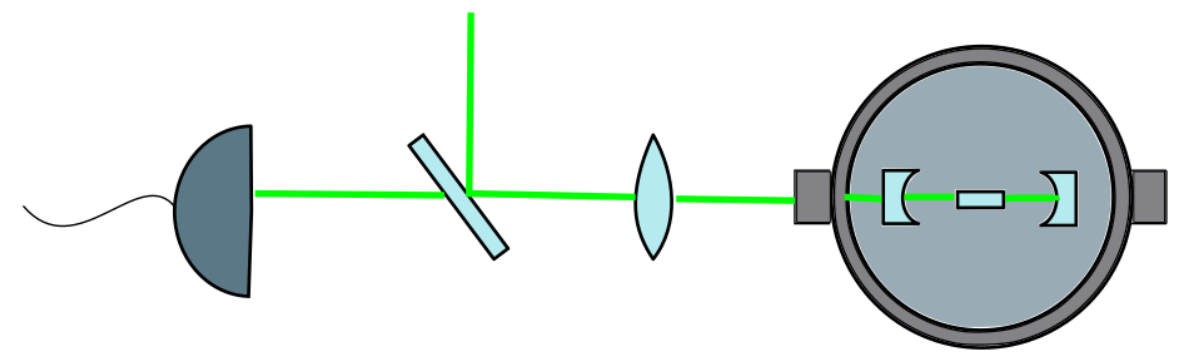

Figure 3.3: Green light comes from the upper part of the figure, is reflected by a mirror, mode matched by a lens and led into the OPO chamber. The reflection of the cavity is measured on a detector and the photocurrent is used for locking.

For the appropriate operation of the OPO, the crystal needs to be temperature stabilized. All the calculations for the expected fluctuations subsection (3.1.3) agrees very well with experiment for the amplitude quadrature; but for phase quadrature, phonon noise comes into play and harms the quantum correlations [41]. This can be controlled by placing the OPO inside a chamber, secure from room atmospheric conditions, and lowering the crystal temperature. To avoid condensation on the crystal's faces, it is important to use a vacuum pump to remove the air inside the chamber.

The temperature control system is depicted on fig (3.4) and described as follows, from bottom to top. A Peltier device is placed between a temperature controlled reservoir on the lower face - here, a copper plate refrigerated by running distilled water - and the crystal on a copper mount on the upper face. Peltiers generates a specific temperature gradient between its two faces for an applied electric voltage. By maintaining its lower face temperature stabilized, the upper face will also be stable if the 
tension applied to it is stable, with a precision on the milikelvin order. Even after removal of the air by the pump vacuum, there is still the possibility that the crystal's face suffer condensation. For this reason, another Peltier is placed above the crystal, and above it, is placed an aluminium foil called trap, covering the whole mount. This second Peltier is responsible for making the aluminium foil's temperature remain lower than the crystal's. If condensation happens, it is going to happen on the foil and not on the crystal.

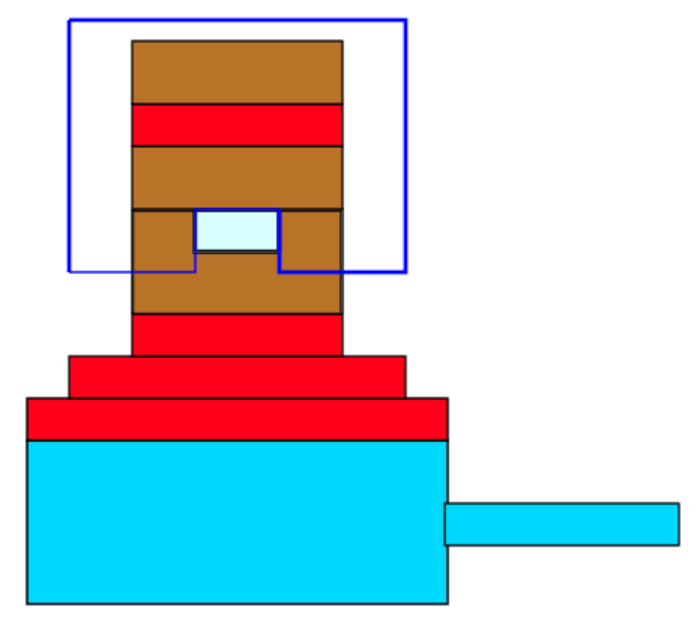

Figure 3.4: Mount for temperature stabilization. The lower part is the water reservoir, the red parts are representing the Peltiers, brown is copper, the crystal is in the middle and the blue contour are representing the place where the trap is located.

\subsubsection{Input mirror}

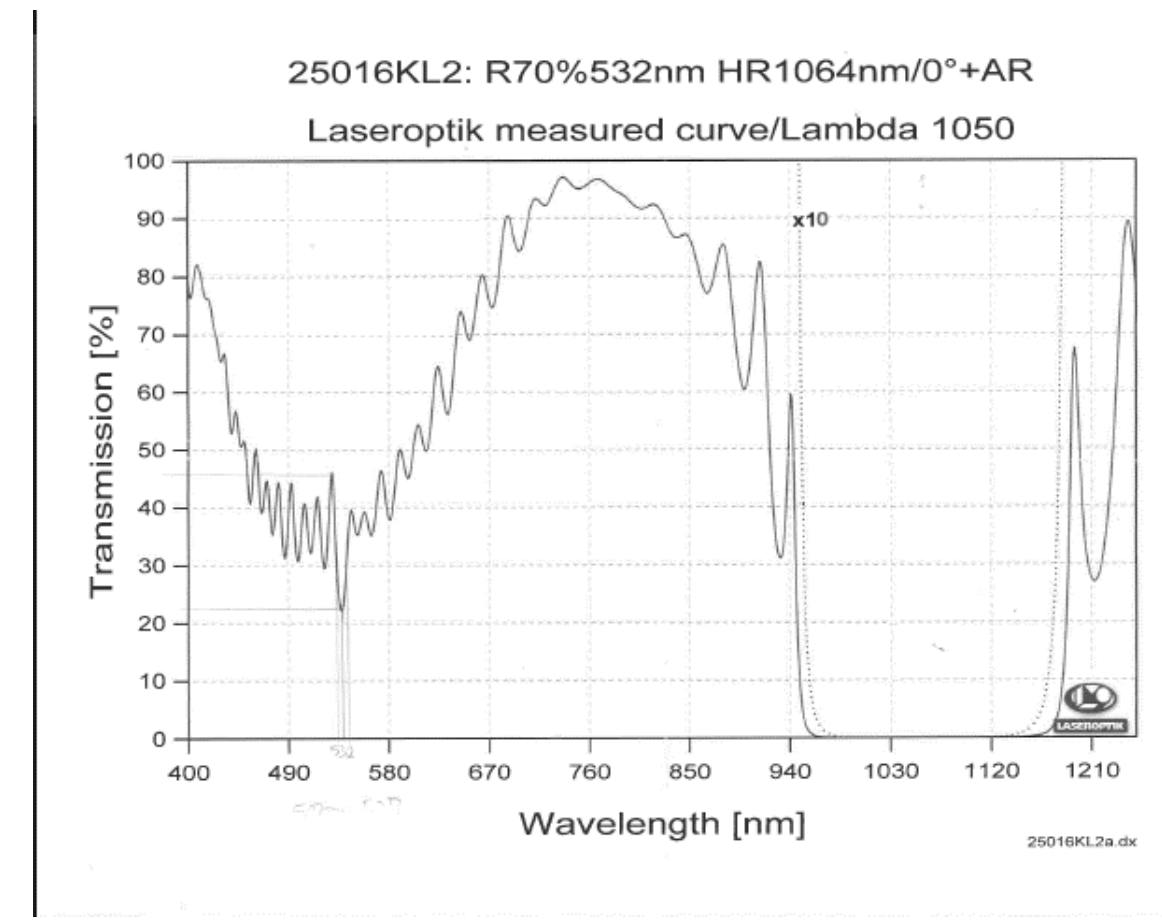

Figure 3.5: Even though the region around $1064 \mathrm{~nm}$ is flat, the region around $532 \mathrm{~nm}$ undergoes fast oscillations, so the mirror's transmission is very sensible to different wavelengths or mirror's conditions. Image provided from the manufacturer.

The OPO mirrors are different from each other, the output was made by ATFilms and the input 
one by Laseroptik. The latter imposes a problem on the experiment, because its transmission changes for different atmospheric conditions. When there is vacuum inside the chamber, its transmission is greater for green, increasing the leaking of the pump. Hence, in order to generate twin beams, one needs to increase the intensity of the beam, resulting in a smaller finesse and a greater threshold power.

In the beginning of 2018, a study performed by undergraduate student Matheus José Oliveira dos Santos resulted in the presented fig (3.6). It plots the mirror's transmission against the relative humidity of air around it. Zero humidity implies a bigger transmission, but excess humidity has the same effect. Therefore, the minimum for the transmission is at some middle point.

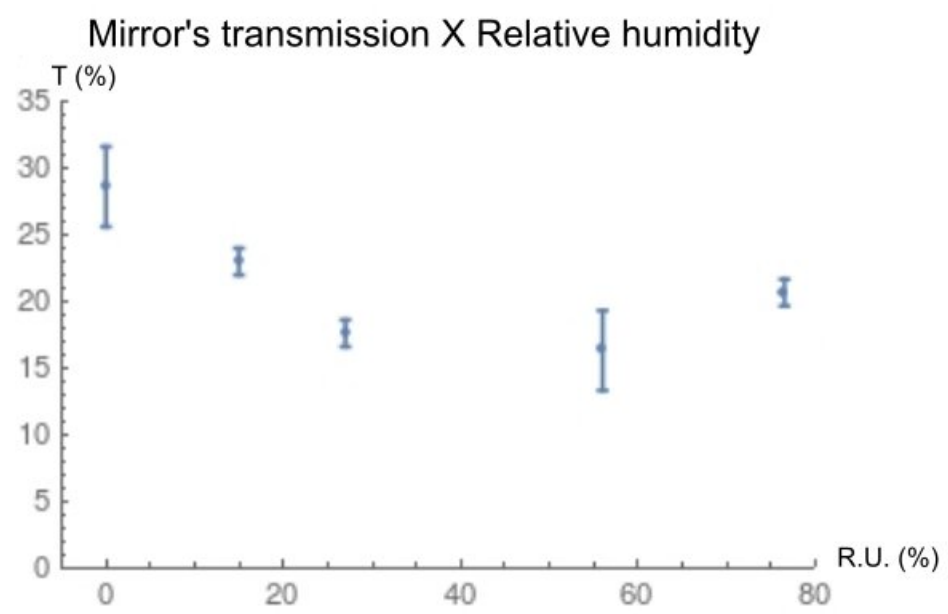

Figure 3.6: Transmission of the mirror for different humidity levels.

Later, an experiment done with Ph.D. student Igor Konieczniak was performed to measure the finesse in relation to humidity [13]. Vacuum was pumped into the chamber, and then it was opened so that ambient humidity could mix with the OPO's. Results are shown in fig (3.7). Time zero in the figure corresponds to the time the chamber was opened. Again, there is a maximum for finesse at some middle point between no humidity and excess humidity.

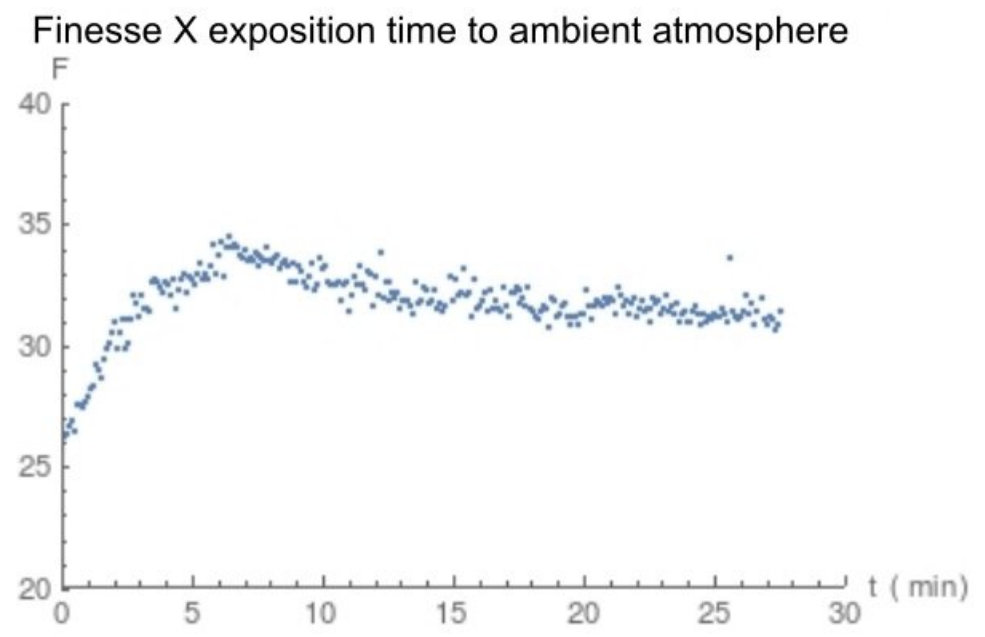

Figure 3.7: Finesse for exposition time to ambient atmosphere.

To maintain the experiment on the best we adopted a procedure of: using the vacuum pump for 30 minutes, expose to OPO to room atmosphere for 30 more minutes and close the chamber. On this manner, we are close to the humidity that gives us the optimal behaviour of the input mirror. However, temperatures below $0{ }^{\circ} \mathrm{C}$ may not be achieved because condensation may happen on the crystal's surfaces. 


\subsubsection{Covariance matrix}

Covariance measurements were performed by maintaing the crystal at $0{ }^{\circ} \mathrm{C}$ under conditions explained on last subsection. The detections were made as explaind on subsection (3.1.4). On the obtained data, an additional rotation in phase space was performed, taking the $a$ indiced quadratures to the $a^{\prime}$ indiced quadratures, by the transformation

$$
\hat{q}_{a} \rightarrow \hat{p}_{a^{\prime}}, \quad \hat{p}_{a} \rightarrow-\hat{q}_{a^{\prime}} .
$$

The one-mode analysis of quadratures has now a simpler form

$$
\hat{P}_{\Omega}=\hat{p}_{s}+i \hat{p}_{a^{\prime}}, \quad \hat{Q}_{\Omega}=\hat{q}_{s}+i \hat{q}_{a^{\prime}} .
$$

The same matrix (2.121) can be retrieved, however, by this rotation, it is now defined as

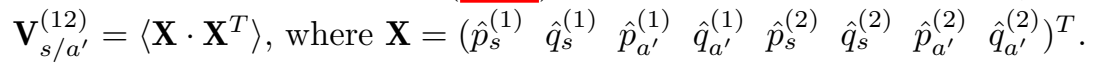

The measurement which yields the best deviation from Duan inequality has the matrices

$$
\begin{aligned}
\mathbf{V}_{s}^{(12)}=\mathbf{V}_{a^{\prime}}^{(12)}= & \left(\begin{array}{cccc}
3.42 & -0.36 & 2.09 & 0.10 \\
-0.36 & 3.68 & -0.36 & -2.18 \\
2.09 & -0.36 & 3.00 & 0 \\
0.10 & -2.18 & 0 & 3.19
\end{array}\right), \\
\mathbf{C}_{s / a^{\prime}}^{(12)} & =\left(\begin{array}{cccc}
0 & 0 & -1.73 & -0.05 \\
0 & 0 & -0.15 & -1.32 \\
1.93 & 0.15 & 0 & -\delta \\
-0.08 & 1.46 & \delta & 0
\end{array}\right),
\end{aligned}
$$

where $\delta$ cannot be measured for Bob's beam, because of the big reflectivity of his cavity, so it is simply written as $\delta$ on the above. Alice's measurements are consistent with $\delta=0$. For the variances and for the correlations relating the same quadratures between different parties, the uncertainties are smaller than 0.2 shot noise units (s.n.u.). For the remaining correlations, uncertainties are 0.05 s.n.u..

These results, however, are not written in the most optimal basis. $\mathbf{C}_{s / a^{\prime}}^{(12)}$ shows correlations between modes $s$ and $a^{\prime}$ of Alice and Bob. It is interesting to apply another rotation in phase space, so all the correlations between parties remain within the same modes. This leads to a matrix $\mathbf{C}_{s / a^{\prime}}^{(12)}$ closer to zero, and all important terms for squeezing are on $\mathbf{V}_{s}^{(12)}$ and $\mathbf{V}_{a^{\prime}}^{(12)}$. When performing teleportation, this rotation will be experimentally applied by the correct gain adjustment (subsection (4.2)). Intuitively, we are finding the rotation to apply which yields us the combinations of quadratures with the best entanglement results.

The angle for which the phase space must be rotated is calculated from the equations for the demodulated signal of section (2.6) where we allow a free parameter $\varphi$ for the phase of the electronic local oscilattor signal (eqn (2.54)), now written as

$$
I_{L O}(t)=I_{0} e^{i \Omega t+\varphi}+I_{0}^{*} e^{-i \Omega t+\varphi} .
$$

Since Alice's amplitude and phase are two separated measurementss, which are mixed with two separated local oscillators signals, we have two parameters, $\varphi_{p}$ and $\varphi_{q}$, which we need to calculate. The measurements with this extra angle (represented by $\hat{X}$ ) are related to the old ones (represented by $\hat{X}^{\prime}$ ) by

$$
\hat{X}_{\cos }=\hat{X}_{\cos }^{\prime} \cos \varphi_{x}+\hat{X}_{\sin }^{\prime} \sin \varphi_{x}, \quad \hat{X}_{\sin }=-\hat{X}_{\cos }^{\prime} \sin \varphi_{x}+\hat{X}_{\sin }^{\prime} \cos \varphi_{x}
$$

where $\hat{X}$ represents either $\hat{P}$ or $\hat{Q}$. Covariances with the new equations must be calculated. In order to find the correct values for $\varphi$, some correlations must be taken to zero. Two of these are the ones relating 
the quadratures $\hat{p}_{s}$ and $\hat{p}_{a^{\prime}}$ between the different stations, both associated with $\hat{P}$. Similar work must be done with the quadratures associated with $\hat{Q}$. The correlations which should be made closer to zero are the ones comparing mode $s$ with mode $a^{\prime}$, present on matrix $\mathbf{C}_{s / a^{\prime}}^{(12)}$,

$$
C\left[\hat{p}_{s}^{(1)}, \hat{p}_{a^{\prime}}^{(2)}\right], \quad C\left[\hat{p}_{a^{\prime}}^{(1)}, \hat{p}_{s}^{(2)}\right], \quad C\left[\hat{q}_{s}^{(1)}, \hat{q}_{a^{\prime}}^{(2)}\right], \quad C\left[\hat{q}_{a^{\prime}}^{(1)}, \hat{q}_{s}^{(2)}\right]
$$

The result is $\varphi_{p}=-0.7(1)$ and $\varphi_{q}=0.57(3)$. After the rotation, we are left with

$$
\begin{aligned}
\mathbf{V}_{s}^{(12)}=\mathbf{V}_{a^{\prime}}^{(12)}= & \left(\begin{array}{cccc}
3.42 & -0.12 & 2.78 & 0.06 \\
-0.12 & 3.68 & -0.39 & -2.59 \\
2.78 & -0.39 & 3.00 & 0 \\
0.06 & -2.59 & 0 & 3.19
\end{array}\right), \\
\mathbf{C}_{s / a^{\prime}}^{(12)} & =\left(\begin{array}{cccc}
0 & 0 & 0.19 & -0.03 \\
0 & 0 & 0.07 & 0.07 \\
-0.05 & -0.06 & 0 & -\delta \\
-0.20 & 0.07 & \delta & 0
\end{array}\right) .
\end{aligned}
$$

These covariance results are closer to the ones we are able to manipulate during experiment since this phase rotation is experimentally performed, as will be explained on chapter 1 .

\subsubsection{Entanglement}

Fig (3.8) shows entanglement measurements taken on two different days of August 2019 under atmospheric conditions explained on subsection (3.2.4). The graphs are plotted as a function of $\sigma$, the ratio between the input power sent to the OPO cavity and the threshold power $\left(\alpha_{0}^{i n}\right)_{\text {min }}^{2}$. It can be calculated from the measured output power $\left|\alpha_{\text {out }}\right|^{2}$ as 442 ,

$$
\begin{gathered}
\left|\alpha_{\text {out }}\right|^{2}=4 \eta\left(\alpha_{0}^{\text {in }}\right)_{\min }^{2}(\sqrt{\sigma}-1), \\
\Rightarrow \sigma=\frac{\left|\alpha_{\text {out }}\right|^{2}}{2 \eta\left(\alpha_{0}^{\text {in }}\right)_{\min }^{2}}
\end{gathered}
$$

where $\eta=g_{0}^{\prime} g^{\prime} /\left(g_{0} g\right)$ is the ratio between losses from the cavity's mirrors and the total losses. From the first line to the second, a first order Taylor expansion was calculated.

During our measurements, intensity is never greater than the $5 \%$ above threshold as shown on the graph. It is clear from the graphs how entanglement on $\hat{P}_{-}$is easier to achieve than entanglement on $\hat{Q}_{+}$. Specially on measurements performed on the first day, $\Delta^{2} \hat{Q}_{+}$has a bigger tendency to increase along with the power. This effect is due to phonon noise, harming phase entanglement measurements for greater power.

The best violation of Duan inequality comes from the second day.

$$
\begin{gathered}
\Delta^{2} \hat{P}_{-}=0.43(16), \quad \Delta^{2} \hat{Q}_{+}=0.85(9), \\
\frac{1}{2}\left(\Delta^{2} \hat{P}_{-}+\hat{Q}_{+}\right)=1.28(18),
\end{gathered}
$$

results which yield a $-1.94 \mathrm{~dB}$ of squeezing. Applying a correction for the losses on the system, one arrives at a value of squeezing of $-4.03 \mathrm{~dB}$. Further details about spurious losses on each part of the experimental setup will be given on subsection (4.4.2).

We can model the obtained squeezing parameters by the known theory. This results in a constant fit for $\Delta^{2} \hat{P}_{-}$and a straight line for $\Delta^{2} \hat{Q}_{+}$, since the latter suffers from phonon noise proportional to the beam's intensity. For the second day of measurements, it is obtained $\Delta^{2} \hat{P}_{-}=0.59(5)$ and $\Delta^{2} \hat{Q}_{+}=-4.9+5.6 * \sigma$. Plots are presented on fig (3.9). 


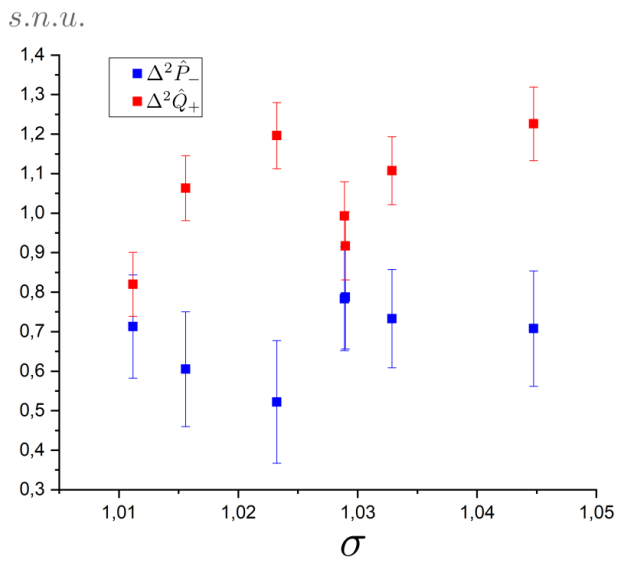

(a) Measurements of first day.

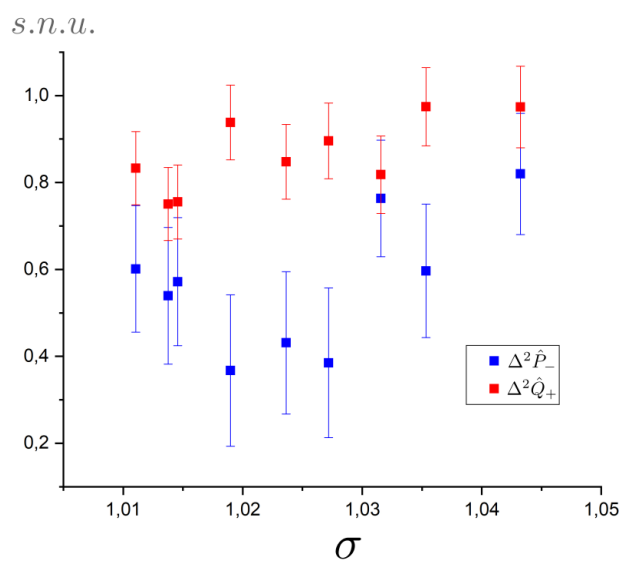

(b) Measurements of second day.

Figure 3.8: Noise compression measurements.

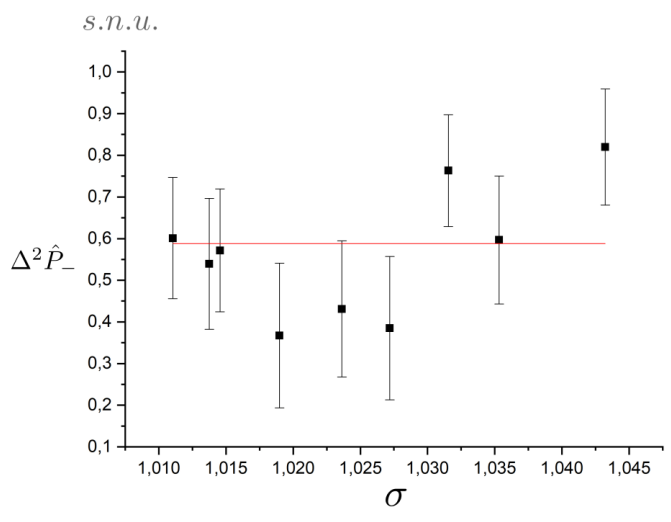

(a) Noise compression on $\Delta^{2} \hat{P}_{-}$.

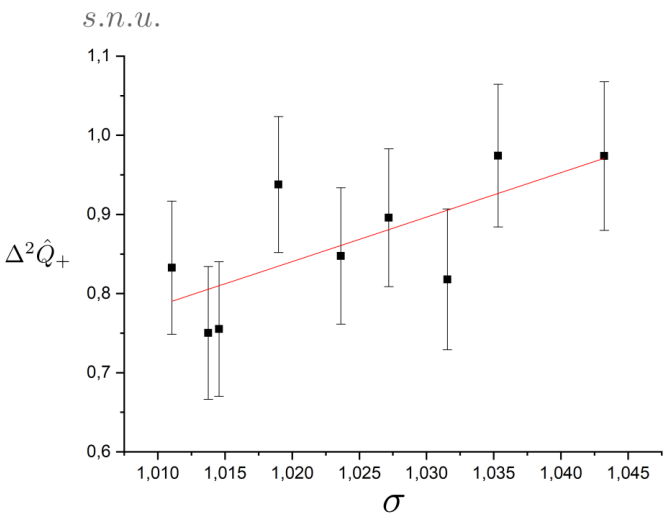

(b) Noise compression on $\Delta^{2} \hat{Q}_{+}$.

Figure 3.9: Squeezing measurements plotted with fitted model.

\subsubsection{Gray-tracking}

The crystal can suffer damage induced by the laser [43] due to long term use. When this happens, the threshold power increases and the transmission for green light is decreased inside the crystal, distorting the lorentzian shape of the transmission of the sweeping OPO. This problem was more evident on the last months of measurement, when a new strategy was necessary: after every measurement day the crystal was moved a bit on a chosen direction. This guarantees that we are using a non damaged part of the crystal each day. A threshold power measurement was performed every day to check the OPO's conditions. 


\section{TELEPORTATION}

Quantum teleportation was first theoretically developed and experimentally achieved for discrete variables [11, 1]. The experiment was performed on polarization states of photons. It made use of a quantum channel generated by entanglement between two different parties we name Alice (indice $A$ ) and Bob (indice $B$ ), sharing the state

$$
\left|\psi_{-}\right\rangle_{A, B}=\frac{1}{\sqrt{2}}\left(|H\rangle_{A}|V\rangle_{B}-|V\rangle_{A}|H\rangle_{B}\right)
$$

where $|H\rangle$ and $|V\rangle$ represents, respectivelly, the horizontal and vertical polarizations. The shown above is one of four maximally entangled states (a Bell state) for this Hilbert space [44]. The other three possible states are

$$
\begin{aligned}
\left|\phi_{+}\right\rangle & =\frac{1}{\sqrt{2}}(|H\rangle|H\rangle+|V\rangle|V\rangle), \\
\left|\phi_{-}\right\rangle & =\frac{1}{\sqrt{2}}(|H\rangle|H\rangle-|V\rangle|V\rangle), \\
\left|\psi_{+}\right\rangle & =\frac{1}{\sqrt{2}}(|H\rangle|V\rangle+|V\rangle|H\rangle),
\end{aligned}
$$

The input state for teleportation,

$$
\left|\psi_{i n}\right\rangle=\alpha|H\rangle+\beta|V\rangle
$$

where $\alpha^{2}+\beta^{2}=1$, is sent to Alice for a joint measurement on the Bell basis (the basis consisting of the four Bell states). The state of the system, considering all three parties is given by

$$
\begin{aligned}
\left|\psi_{i n}\right\rangle \otimes\left|\psi_{-}\right\rangle_{A, B}= & \frac{1}{2}\left[\left|\psi_{-}\right\rangle_{i n, A}\left(-\alpha|H\rangle_{B}-\beta|V\rangle_{B}\right)\right. \\
& +\left|\psi_{+}\right\rangle_{i n, A}\left(-\alpha|H\rangle_{B}+\beta|V\rangle_{B}\right) \\
& +\left|\psi_{-}\right\rangle_{i n, A}\left(\alpha|V\rangle_{B}+\beta|H\rangle_{B}\right) \\
& \left.+\left|\phi_{+}\right\rangle_{i n, A}\left(\alpha|V\rangle_{B}-\beta|H\rangle_{B}\right)\right]
\end{aligned}
$$

where $\otimes$ represent the vector product. It can be seen that, with one fourth probability, the outcome of this measurement will be $\left|\psi_{-}\right\rangle_{i n, A}$, which will project Bob's beam into the initial state (up to a global phase). For the other results of Alice's measurements, Bob needs to apply an unitary operator to retrieve the desired state. For the teleportation process to be successful, Alice must inform Bob about her measurement outcome, so he can act on the state he possesses correctly.

The performance on continuous variables was achieved one year later [3], with experimental characteristics more similar to the one of the present thesis. Both generates entanglement by using an OPO and works with the quadratures $\hat{P}$ and $\hat{Q}$ of coherent beams. However, on the cited experiment, the squeezed fields generated by the OPO are vacuum (quadratures' mean values equal zero) while the present work generates bright beams.

Past contributions to the setup of this thesis explored teleportation between fields on different frequencies of light [13]. The mathematics and physics of this process are explained on this chapter until section (4.4), with increasing levels of complexity. The protocol is explained first on the ideal case and later the losses and imperfections of the system are taken into account in order to predict the expected results.

A novelty brought to the experiment is the analysis of each of the sideband modes during the process. Section (4.5) makes the extension to this new form of analysis, consisting of original results. The remaining sections explains how to evaluate the success of a teleportation measurement and present the expected results considering the specific characteristics of this experiment. 


\subsection{Basic notions}

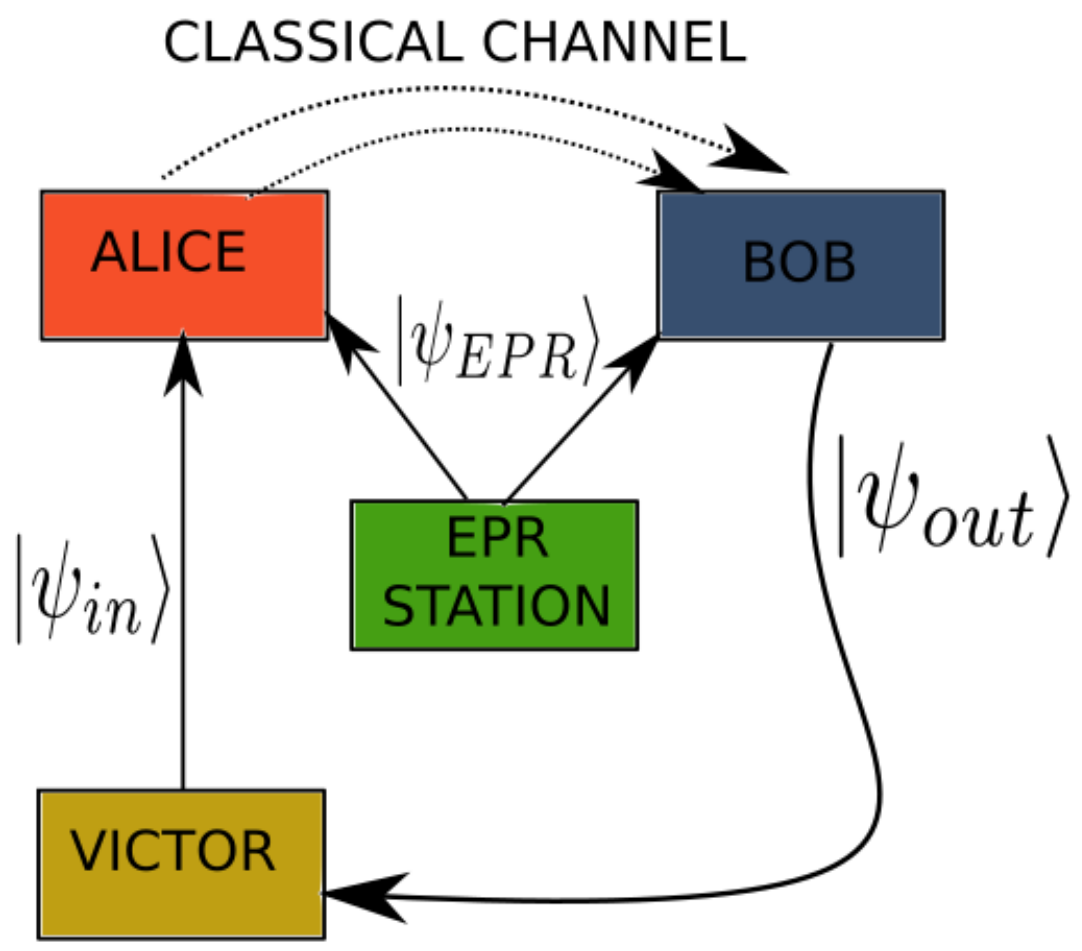

Figure 4.1: Pictorial description of teleportation

Fig (4.1) is a schematic representation of the general process, performed with three characters

- Victor: begins the protocol by preparing the input state for teleportation. He is responsible to check if the experiment was successful the end of the protocol, calculating the quality by comparison between input and output states.

- Alice: measures Victor's state by combining it with the state she possesses and communicates the result to Bob via classical channel.

- Bob: creates the output state, as close as possible to Victor's initial state, using Alice's information about her measurement.

For this process to work properly, Alice and Bob must share an entangled state. On continuous variables, it is an EPR state as the output of an OPO (section (3.1)). On discrete variables, this is a Bell state. A priori, there is no rule for which kind of state Victor must create, as long as it is agreed in what mode the information is encoded (polarization, sidebands etc). A fully functional teleportation protocol is one able to teleport any given state of the chosen Hilbert space.

The input state created by Victor is sent to Alice for a joint measurement along with her part of the entangled state. On discrete variables, this is done by measuring both states in a Bell basis. On continuous variables, it is done by interfering Alice and Victor's beams. The obtained measurement results are sent to Bob. Since the quantum correlations between his beam and Alice's are known, he can infer the operation required to retrieved Victor's initial state.

This process resembles a cryptographic protocol. Suppose, for instance, that Victor wants to send an information to Bob by a secure channel. He can mix his state with Alice's so whoever access the 
measurement results cannot tell what is the input state. Victor scrambled his information by mixing it with another state. Bob is the only one who has the key to decode the message, since the entanglement he shares with Alice allows him to infer how this scrambling was done.

\subsection{Proposal}

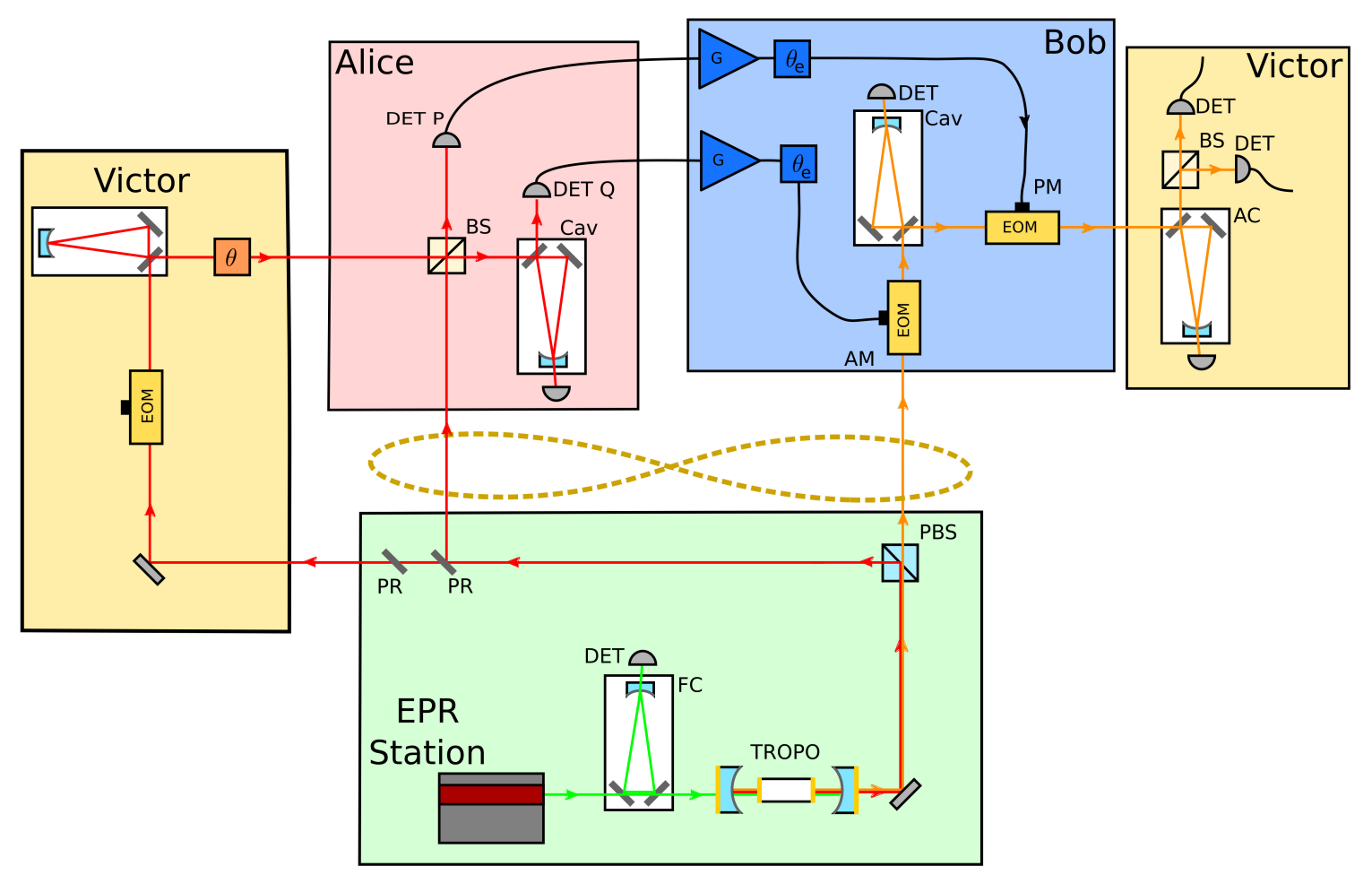

Figure 4.2: Experimental realization of the pictorial description in fig (4.1).

The work of this thesis (schematically represented on fig (4.2)) uses an above threshold OPO generating twin beams of different colors and it possesses the ability to create sidebands with different energies, different from other works on continuous variables teleportation.

The protocol begins at the EPR station, with the laser light firstly directed to a filter cavity (subsection (3.2.1)) and then to the OPO chamber to generate the twin beams, which are divided between Alice and Bob by means of a polarized beam splitter. For the beginning of this chapter, it will be considered that the correlations between signal and idler are infinite, so the physics of the process can be better visualized. For the same reason, this first presentation of teleportation will deal with single mode analysis, using quadratures $\hat{P}$ and $\hat{Q}$.

On the EPR station (green box in fig (4.2)), a triply resonant OPO built with a type-II crystal generates entanglement between the beams. Alice's part of the state is sent to a $99 \%$ partially reflective mirror ( $P R$ on the figure), where the transmission is further attenuated by another $P R$ and sent to Victor, while the reflection is sent to her station. This guarantees that both parties are working on the exact same wavelength. By keeping most of the energy on Alice's part, the quantum correlations present on signal and idler are stronger on Alice's beam than Victor's. Victor creates the input state by using an EOM (section (2.7)), and he is also able to generate asymmetries on his sidebands by means of an optical cavity locked on a position suppressing one of them. This state is then mixed with Alice's by an 
interferometric process and both quadratures are measured: $\hat{P}$ by sending light to a detector and $\hat{Q}$ by using a cavity to rotate the noise ellipse (subsection (2.8.2)). The measurement results must be amplified and sent to Bob, that will reconstruct the state performing the necessary operations. At last, Victor checks if the teleportation was successful.

\subsubsection{EPR station}

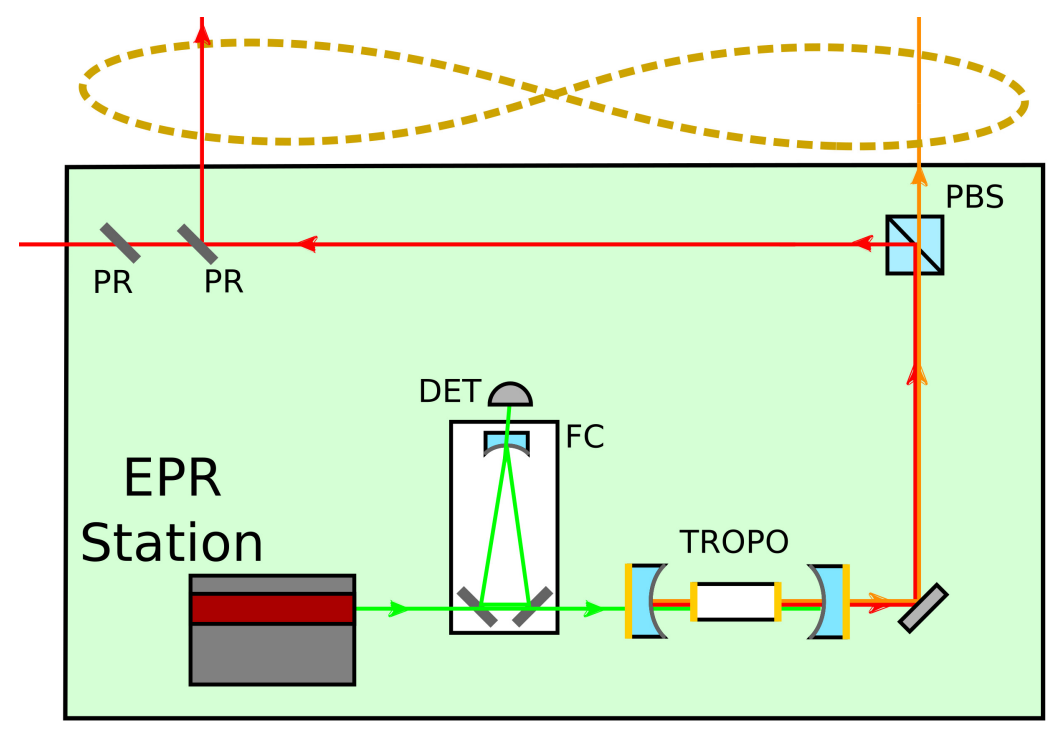

Figure 4.3: EPR station, containing the laser, a filter cavity and the OPO chamber. Green line represents the pump field, red the beam that is going to Alice's side and orange is Bob's beam.

Fig (4.3) represents the setup presented on section (3.2), the so called EPR station cointaing the pump field (an Innolight Diabolo green laser), a filter cavity (subsection (3.2.1)) and the OPO chamber (subsection (3.2.2)). The output states of the OPO are associated with the following Heisenberg picture operators (subsection (2.5.1))

$$
\begin{aligned}
& \hat{a}_{A}=\alpha_{A}+\delta \hat{a}_{A}, \\
& \hat{a}_{B}=\alpha_{B}+\delta \hat{a}_{B},
\end{aligned}
$$

where the subscript $A$ represents the mode sent to Alice and $B$ the one sent to Bob.

Squeezing is present on the quadratures' combinations (subsection (3.1.3))

$$
\begin{array}{r}
\delta \hat{P}_{-}=\frac{1}{\sqrt{2}}\left(\delta \hat{P}_{B}-\delta \hat{P}_{A}\right), \\
\delta \hat{Q}_{+}=\frac{1}{\sqrt{2}}\left(\delta \hat{Q}_{B}+\delta \hat{Q}_{A}\right) .
\end{array}
$$

Their variances will violate Duan's inequality (eqn (3.58)).

\subsubsection{Victor creates the input state}

The state to be teleported is built by Victor from the $0.01 \%$ light transmitted through the partially reflective mirrors of fig (4.3). This beam will later be interfered with Alice's in a Mach-Zehnder.

Victor needs to operate three devices in order to create the desired state: the EOM, the cavity and the locking position of the interferometer, all present on fig (4.4).

- The EOM 


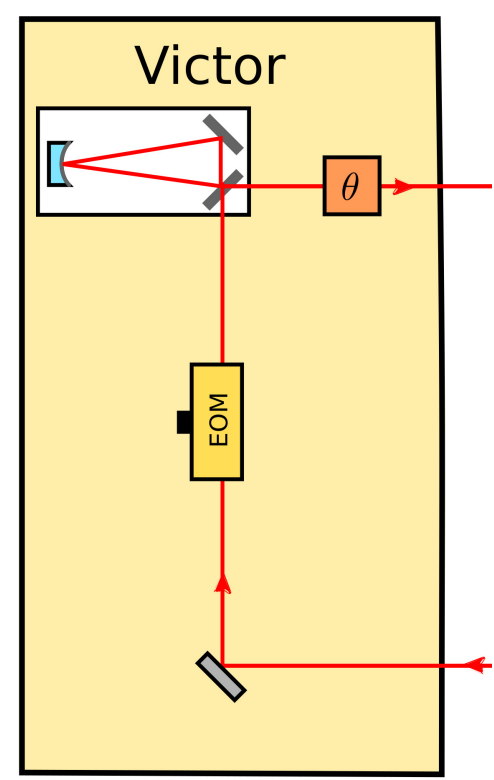

Figure 4.4: Victor creates his input state by using his EOM, his cavity and the locking position of the interferometer

This device is a crystal with the property of changing its refractive index when an electric voltage is applied. For the experiment, it was chosen to modulate Victor's phase on a $15 \mathrm{MHz}$ frequency, setting the sidebands to this position on frequency space. Every party — Victor, Alice and Bob — must agree that information will be encoded on this specific frequency. The phase $\phi_{m}$ of modulation is also an information that must be shared between all parties.

- The cavity

The cavity is $51 \mathrm{~cm}$ long, its finesse is 40 and the bandwidth is $14,7 \mathrm{MHz}$. Its construction permits it to be resonant with one of the sidebands, but not with the other, adding an asymmetry on the prepared state. The intensity transmittance of a cavity is known as a Lorentzian (subsection (2.8.1))

$$
I=\frac{1}{1+\left(\frac{2 \omega}{\gamma}\right)^{2}},
$$

where the amplitude is normalized to 1 and $\omega$ is making the role of the detuning on eqn (2.92). If the cavity is resonant on one of the sidebands, located at the $15 \mathrm{MHz}$ frequency, then

$$
I=\frac{1}{1+\left(\frac{30}{14,7}\right)^{2}} \approx 0.2 .
$$

In conclusion, by locking the cavity at $20 \%$ transmission of the peak, only one of the sidebands is resonating while the other is reflected and sent to experiment as the input state.

For simplicity, we will first restrict ourselves to symmetric sidebands' states, equivalent to not using Victor's cavity. On section (4.5), the extension to general states will be made.

- The relative phase of the interferometer

Defining the locking position is defining the quadratures that are going to be modulated. Using the EOM, Victor wrote a signal at the beam's phase, but if the desired input state is an amplitude modulated one, the interferometer needs to be adjusted as explained in subsection (2.7). The locking is done by the side of fringe technique described on appendix (A .1). 
In summary, Victor creates his state on the following manner. He first modulates the beam's phase, creating equal sidebands around the carrier. He then decides if he locks his cavity at $20 \%$ the peak's height to obtain a single sideband state. After that, he chooses the position of the interferometer lock to choose which quadratures are modulated and by what amount.

If the state sent to Victor is associated to the operator

$$
\hat{a}_{V}=\alpha_{V}+\frac{1}{2}\left(\delta \hat{P}_{V}+i \delta \hat{Q}_{V}\right)
$$

then, after the information is imprinted on the beam, it will be

$$
\hat{a}_{V}=\alpha_{V}+\frac{1}{2}\left[\delta \hat{P}_{V}+A_{M}+i\left(\delta \hat{Q}_{V}+P_{M}\right)\right]
$$

where $A_{M}$ and $P_{M}$ represents the amplitude and phase modulation performed by the EOM. Using the notation of subsection (2.7.2), we have

$$
A_{M}=\sqrt{p_{s}^{2}+q_{a}^{2}}, \quad P_{M}=\sqrt{q_{s}^{2}+p_{a}^{2}} .
$$

All of the sidebands' information considered here are on $15 \mathrm{MHz}$.

\subsubsection{Alice}

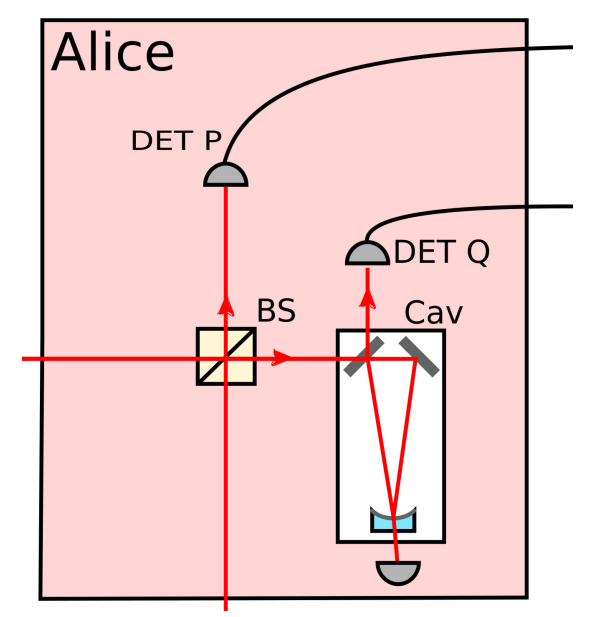

Figure 4.5: Alice's station. The interferometer is completed and she proceeds to measure the beam's phase and amplitude.

The interferometer is completed at Alice's station, where both outputs are sent to detectors. One of them measures directly the beam's intensity, proportional to the amplitude quadrature. The other beam splitter output is first sent to a cavity, locked by the side of fringe method of locking (appendix (A 1)), so the noise ellipse is rotated in the desired way for the measurement of this beam to give information about the phase quadrature. The results of measurements must be amplified and then sent to Bob.

The beam splitter outputs are

$$
\begin{aligned}
& \hat{a}_{p}=\frac{1}{\sqrt{2}}\left[\alpha_{V}-\alpha_{A}+\frac{1}{2}\left[\delta \hat{P}_{V}-\delta \hat{P}_{A}+A_{M}+i\left(\delta \hat{Q}_{V}-\hat{Q}_{A}+P_{M}\right)\right]\right] \\
& \hat{a}_{q}=\frac{1}{\sqrt{2}}\left[\alpha_{V}+\alpha_{A}+\frac{1}{2}\left[\delta \hat{P}_{V}+\delta \hat{P}_{A}+A_{M}+i\left(\delta \hat{Q}_{V}+\hat{Q}_{A}+P_{M}\right)\right]\right],
\end{aligned}
$$

where the $\hat{P}$ quadrature is going to be measured on $\hat{a}_{p}$ and the $\hat{Q}$ quadrature is going to be measured on $\hat{a}_{q}$. As a result, DET P on fig (4.5) will only measure $\delta \hat{P}_{V}-\delta \hat{P}_{A}+A_{M}$ while DET Q will only measure $\delta \hat{Q}_{V}+\delta \hat{Q}_{A}+P_{M}$. The acquired information will be split in two: one part will be sent to Bob, and the 
other will go through a demodulation chain to be mixed with an electronic local oscillator (subsection (2.7)).

Along with the photocurrents, the modulation phase $\phi_{m}$ of the modulation must also be sent to the demodulation chain. This phase is an important reference and it is needed for the photocurrents to be correctly divided into its sine and cosine parts. After demodulation, we will have four Alice measurements: the sine and cosine parts of DET P results (that will be called $P_{\text {cos }}^{A}$ and $P_{\text {sin }}^{A}$ ), and the sine and cosine parts of DET Q results (called $Q_{\mathrm{cos}}^{A}$ and $Q_{\mathrm{sin}}^{A}$ ). Alice's measurements' mean values results are

$$
\begin{aligned}
P_{\mathrm{cos}}^{A} & =A_{M} \cos \phi_{m}, \\
P_{\mathrm{sin}}^{A} & =A_{M} \sin \phi_{m}, \\
Q_{\mathrm{cos}}^{A} & =P_{M}\left(\cos \phi_{m} \sin \alpha-\sin \phi_{m} \cos \alpha\right), \\
Q_{\mathrm{sin}}^{A} & =P_{M}\left(\sin \phi_{m} \sin \alpha+\cos \phi_{m} \cos \alpha\right),
\end{aligned}
$$

where $\alpha$ is an angle present due to Alice's cavity, imposing a different output for each sideband [33]. This term is related to the reflection of the carrier and of the sideband at $\Omega$ like shown below.

$$
\begin{aligned}
& \sin \alpha=\operatorname{Im}\left[r_{A}^{*}(0) r_{A}(\Omega)\right], \\
& \cos \alpha=\operatorname{Re}\left[r_{A}^{*}(0) r_{A}(\Omega)\right],
\end{aligned}
$$

only valid when the cavity is perfectly locked on the quadratures conversion position (subsection (2.8.2)). When the modulation phase information $\phi_{m}$ is not shared between modulation and demodulation, the measurement results are as explained on appendix $(\mathbb{a})$.

These equations tell us that measuring the amplitude modulation is straighforward, being directly related to Alice's photocurrents. However, the phase modulation measurements will suffer a mixture from the sine and cosine quadratures due to the cavity. This can be corrected a posteriori by a proper measurement of the cavity's parameters being considered in the analysis.

In order to calculate the input state parameters $A_{M}$ and $P_{M}$, we simply calculate

$$
A_{M}=\sqrt{\left(P_{\mathrm{cos}}^{A}\right)^{2}+\left(P_{\mathrm{sin}}^{A}\right)^{2}}, \quad P_{M}=\sqrt{\left(Q_{\mathrm{cos}}^{A}\right)^{2}+\left(Q_{\mathrm{sin}}^{A}\right)^{2}} .
$$

\subsubsection{Bob}

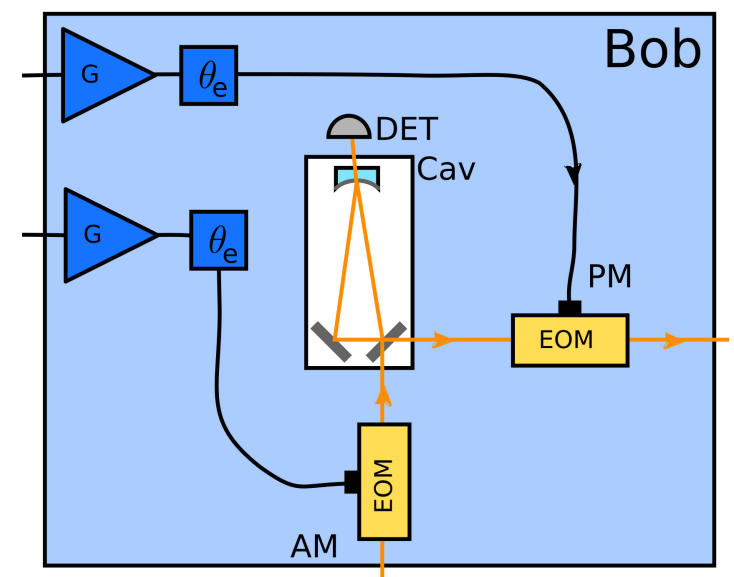

Figure 4.6: Bob receives Alice's information about her measurement and acts on his beam accordingly so Victor's input state can be reconstructed.

Bob receives the other output of the OPO and the non-modulated Alice's photocurrent, that is, $P^{A}=P_{\cos }^{A} \cos \Omega t+P_{\sin }^{A} \sin \Omega t$ and $Q^{A}=Q_{\cos }^{A} \cos \Omega t+Q_{\sin }^{A} \sin \Omega t$. This information will be amplified by 
an adjustable gain $g_{p, q}$ (The indices $P, Q$ indicating the results from the detectors measuring $\hat{a}_{p}$ and $\hat{a}_{q}$ ) and shifted by a controllable phase $\phi_{p, q}$. This phase shift is the experimental realization of the rotation on phase space performed on subsection (3.2.5), used on the covariance matrix to define the quadratures on the basis with greater squeezing. The gain and phase delay will be designed by the parameter $\Gamma$, that hereafter will be called gain

$$
\Gamma_{p, q}=g_{p, q} e^{i \phi_{p, q}}
$$

It must be called attention to the fact that the EOMs on the experiment only modulate phase. On the beginning of this project, Bob had an EOM to modulate the phase of his beam (according to Alice's phase measurement) and one EOM to modulate the amplitude (according to Alice's amplitude measurement). However, it was noticed that the amplitude modulation was not perfect, and the phase always ended up modulated by an undesired amount [13]. The solution was to build a cavity to Bob and use two EOMs, one before and another one after the cavity as shown on fig (4.6).

The first EOM will receive Alice's phase information and act on Bob's beam,

$$
\hat{a}_{1}=\alpha_{B}+\frac{1}{2}\left[\delta \hat{P}_{B}+i \delta \hat{Q}_{B}+i \Gamma_{q}\left(\delta \hat{Q}_{V}+\delta \hat{Q}_{A}+P_{M}\right)\right] .
$$

Using $\delta \hat{Q}_{B}+\delta \hat{Q}_{A} \rightarrow 0$ (eqn (4.10) ) and $\Gamma_{q}=1$, we have

$$
\hat{a}_{1}=\alpha_{B}+\frac{1}{2}\left[\delta \hat{P}_{B}+i\left(\delta \hat{Q}_{V}+P_{M}\right)\right] .
$$

The cavity then swaps quadratures

$$
\hat{a}_{2}=\alpha_{B}+\frac{1}{2}\left[\delta \hat{Q}_{V}+P_{M}+i \delta \hat{P}_{B}\right],
$$

and on the phase of this beam, Alice's amplitude information is put,

$$
\hat{a}_{3}=\alpha_{B}+\frac{1}{2}\left[\delta \hat{Q}_{V}+P_{M}+i\left[\delta \hat{P}_{B}-\Gamma_{p}\left(\delta \hat{P}_{A}-\delta \hat{P}_{V}-A_{M}\right)\right]\right] .
$$

Again, considering perfectly adjusted $\Gamma_{p}=1$ and infinite squeezing $\delta \hat{P}_{B}-\delta \hat{P}_{A} \rightarrow 0$ (eqn (4.9)), the final output is

$$
\hat{a}_{\text {out }}=\alpha_{B}+\frac{1}{2}\left[\delta \hat{Q}_{V}+P_{M}+i\left(\delta \hat{P}_{V}+A_{M}\right)\right]
$$

This retrieves Victor input state up to a quadrature change $\hat{P} \leftrightarrow \hat{Q}$ (eqn (4.14)).

\subsubsection{Victor again}

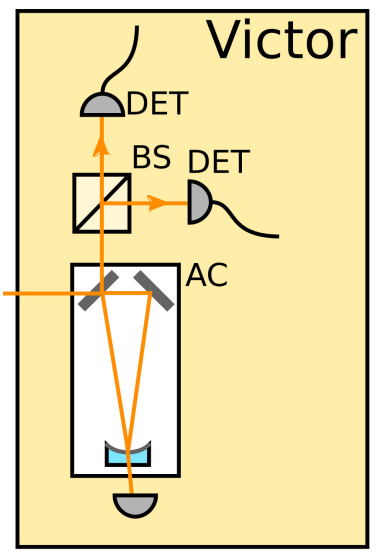

Figure 4.7: Victor checks if teleportation was successful. 
The last step of the process is to check if the teleportation worked. For this, Victor needs a cavity, the only one in the experiment that is not locked. This cavity must scan while the detectors take measurements at every detuning value. During $750 \mathrm{~ms}$, the acquisition can make 450000 measurements, a $600 \mathrm{kHz}$ measurement rate. This station is schematically represented on fig (4.7).

Victor's photocurrent will also be demodulated, allowing us to measure all terms on eqns (2.113) and (2.114). By fitting the cited equation to the mean values' result, we are able to relate the populations of Victor's quadrature with Alice's measurements. Cosine demodulation is shown below.

$$
\left(\begin{array}{c}
p_{s}^{V} \\
q_{a}^{V} \\
p_{a}^{V} \\
q_{s}^{V}
\end{array}\right)=\left(\begin{array}{cccc}
-g_{p} \sin \phi_{p} & 0 & -g_{q} \cos \phi_{q} \sin \beta & g_{q} \cos \phi_{q} \cos \beta \\
0 & -g_{p} \sin \phi_{p} & -g_{q} \cos \phi_{q} \cos \beta & -g_{q} \cos \phi_{q} \sin \beta \\
g_{p} \cos \phi_{p} & 0 & -g_{q} \sin \phi_{q} \sin \beta & g_{q} \sin \phi_{q} \cos \beta \\
0 & g_{p} \cos \phi_{p} & -g_{q} \sin \phi_{q} \cos \beta & -g_{q} \sin \phi_{q} \sin \beta
\end{array}\right)\left(\begin{array}{c}
P_{\cos }^{A} \\
P_{\sin }^{A} \\
Q_{\cos }^{A} \\
Q_{\sin }^{A}
\end{array}\right)
$$

and when the gains are perfectly adjusted $\left(g_{p, q}=1\right.$ and $\left.\phi_{p, q}=0\right)$

$$
\left(\begin{array}{l}
p_{s}^{V} \\
q_{a}^{V} \\
p_{a}^{V} \\
q_{s}^{V}
\end{array}\right)=\left(\begin{array}{cccc}
0 & 0 & -\sin \beta & \cos \beta \\
0 & 0 & -\cos \beta & -\sin \beta \\
1 & 0 & 0 & 0 \\
0 & 1 & 0 & 0
\end{array}\right)\left(\begin{array}{c}
P_{\cos }^{A} \\
P_{\sin }^{A} \\
Q_{\cos }^{A} \\
Q_{\sin }^{A}
\end{array}\right)
$$

where $\beta$ in both matrices is related to Bob's cavity reflection terms

$$
\begin{aligned}
& \sin \beta=\operatorname{Im}\left[r_{B}^{*}(0) r_{B}(\Omega)\right], \\
& \cos \beta=\operatorname{Re}\left[r_{B}^{*}(0) r_{B}(\Omega)\right] .
\end{aligned}
$$

Victor's measurement of Alice's amplitude modulation will appear on his $\hat{Q}$ quadrature while his measurements of Alice's phase modulation will appear on his $\hat{P}$ quadrature. Victor will also measure his amplitude information directly, but his phase information will be mixed because of Bob's cavity. His mean values measurements with respect to the input state are

$$
\begin{array}{lc}
p_{s}^{V}= & P_{M}\left(\cos \phi_{m} \cos (\alpha+\beta)+\sin \phi_{m} \sin (\alpha+\beta)\right), \\
q_{a}^{V}= & P_{M}\left(-\cos \phi_{m} \sin (\alpha+\beta)+\sin \phi_{m} \cos (\alpha+\beta)\right), \\
p_{a}^{V}= & A_{M} \cos \phi_{m}, \\
q_{s}^{V}= & A_{M} \sin \phi_{m} .
\end{array}
$$

We retrieve the values of the initial modulations by calculating

$$
P_{M}=\sqrt{\left(p_{s}^{V}\right)^{2}+\left(q_{a}^{V}\right)^{2}}, \quad A_{M}=\sqrt{\left(p_{a}^{V}\right)^{2}+\left(q_{s}^{V}\right)^{2}}
$$

By comparing the measured $A_{M}$ and $P_{M}$ on above Victor's measurementes with Alice's measurements on eqns (4.18) to (4.21), teleportation success can be calculated.

\subsection{Gain adjustment}

For the experiment to work, the value of $\Gamma$ must be well adjusted. This task is performed by making use of the $I R$ field from the Diabolo. The optical path taken by this field is shown in fig (4.8). Its difference from fig (4.2) is located on the EPR Station (on fig (4.8) called Correlations). Instead of creating two beams sharing quantum correlations, this station will create classical correlations between states. The EOM inside a Mach-Zehnder interferometer can modulate both quadratures (as seen on subsection (2.7)) of the beam which will later be equally divided between Alice and Bob. The state of 


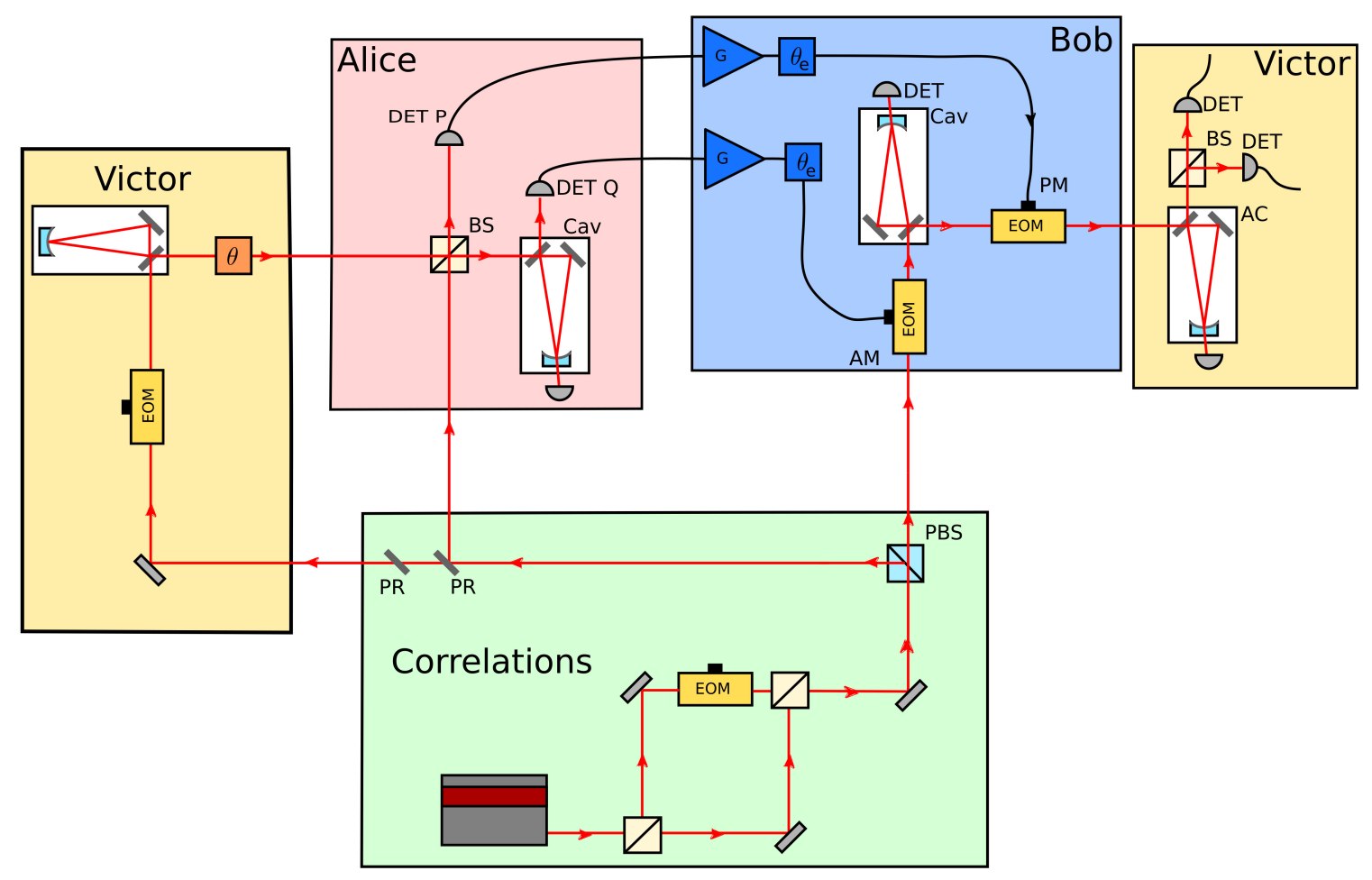

Figure 4.8: Setup for Diabolo's IR field.

each party will be classically correlated with one another because they will have the same mean values of quadratures, but they are not entangled.

For the gain adjustment, Victor station will be left unused because there is no need to create an input state at this point. Alice's quadratures will be measured and the generated photocurrent will be used as input for Bob's EOMs. He will try to disappear with the information on his sidebands by adjusting the gain and phase of the received electronic signal. When Bob can only measure vacuum fluctuations on his beam, compensating the modulation performed on the beginning, the gain is said to be perfectly adjusted. By this method, we are able to remove $30 \mathrm{~dB}$ of modulation.

The gain adjustment is an energy dependent process. Alice's sideband measurement is proportional to the beam's amplitude $\alpha_{A}$ (eqn (2.49)), and the EOM on Bob's station will build a state proportional to the beam's amplitude $\alpha_{B}$ (section (2.7)). So, when adjusting the gains, an intensity value must be chosen for the experiment to be performed, setting $\alpha_{A, a d}$ and $\alpha_{B, a d}$, the amplitudes for which teleportation works. The position of perfect adjustment, $\Gamma=1$, is the point where the carrier's amplitudes are compensated,

$$
\Gamma=\frac{\alpha_{A} \alpha_{B}}{\Gamma_{a d}},
$$

where $\Gamma_{a d}=\alpha_{A, a d} \alpha_{B, a d} \approx\left|\alpha_{A, a d}\right|^{2} \approx\left|\alpha_{B, a d}\right|^{2}$, approximations taken because $\alpha_{A}$ and $\alpha_{B}$ are experimentally set at similar values. Measurements at different gains are obtained by varying the intensity of the laser when collecting data.

To check if each of Alice's signals is being correctly sent to Bob, the results of table 4.1 were taken. It shows measurements taken with a spectral analyser at $15 \mathrm{MHz}$ of output Victor's quadratures. Quadratute $\hat{P}$ is measured by leaving his cavity unused, acting only as a mirror; and $\hat{Q}$ is measured by locking his cavity at $\Delta=1 / 2$ (subsection (2.8.2)). The table shows that blocking Alice's DET Q affects 
the $\hat{P}$ quadrature of Victor; and by blocking DET P, it is Victor's $\hat{Q}$ quadrature that is affected. This is expected by eqn (4.30), that tells us that our protocol swaps the quadratures' information at the output.

\begin{tabular}{c||c|c} 
& Victor P $\left(10^{-6} V\right)$ & Victor Q $\left(10^{-6} V\right)$ \\
Electronic noise & $23.4(3)$ & $25.4(3)$ \\
Shot noise & $66.8(7)$ & $66.8(7)$ \\
Sum & $133(9)$ & $105(9)$ \\
Blocked Alice Q & $83.1(9)$ & $99(9)$ \\
Blocked Alice P & $135(9)$ & $75.7(9)$
\end{tabular}

Table 4.1: Victor's measurements of the electronic noise (result of taking a measurement with no light), shot noise (subtraction of both detectors), sum of the detectors, information from DET Q of fig (4.8) being blocked and information from DET P of figure being blocked.

After calibration is concluded, the initial correlations can be turned off and Victor can now create an input state for classical teleportation to be performed. Under these conditions, there are no entangled fields, imposing a limit on the performance of the protocol, as will be seen on subsection (4.6.1). Nonetheless, this is a good test of the system, telling us if the gains are properly adjusted and if every equipment is working as they should.

If the classical teleportation results turn out as expected, the green Diabolo field is turned on and the teleportation protocol as explained before is performed. When Alice and Bob share quantum correlations, the performed protocol is called quantum teleportation.

\subsection{Realistic quantum teleportation}

Before the presentation of the more general two-mode teleportation protocol, it is important to include more realism to the described equations. The first step is to quantify the effect of a finite amount of squeezing to the studied scheme, issue addressed on subsection (4.4.1). Spurious losses due to imperfect detection efficiencies and passage trough optical components will also negatively impact the final result, as will be shown on subsection (4.4.2).

\subsubsection{Finite squeezing}

Section (4.2) describes quantum teleportation considering infinite squeezing, the case where $\delta \hat{P}_{-}=0$ and $\delta \hat{Q}_{+}=0$. Despite being a intuitive first approach, infinitely squeezed quadratures are non physical. This subsection will develop the calculations for a more realistic scenario, involving a finite amount of correlation [46, 47].

Consider two single-mode vacuum squeezed states, 1 and 2, with quadratures

$$
\begin{array}{cc}
\delta \hat{Q}_{1}=e^{-r} \delta \hat{Q}_{1}^{0}, & \delta \hat{P}_{1}=e^{r} \delta \hat{P}_{1}^{0}, \\
\delta \hat{Q}_{2}=e^{s} \delta \hat{Q}_{2}^{0}, & \delta \hat{P}_{2}=e^{-s} \delta \hat{P}_{2}^{0},
\end{array}
$$

where the superscript 0 denotes the shot noise level for the quadrature's fluctuation. These equations describe states equivalent to the one in eqn (2.26). Each one is squeezed in a different quadrature with a different squeezing parameter. Alice and Bob's states are modeled as the outputs of sending both of these states in a beam splitter.

$$
\begin{array}{ll}
\delta \hat{Q}_{A}=\frac{1}{\sqrt{2}}\left(e^{-r} \delta \hat{Q}_{1}^{0}+e^{s} \delta \hat{Q}_{2}^{0}\right), & \delta \hat{P}_{A}=\frac{1}{\sqrt{2}}\left(e^{r} \delta \hat{P}_{1}^{0}+e^{-s} \delta \hat{P}_{2}^{0}\right), \\
\delta \hat{Q}_{B}=\frac{1}{\sqrt{2}}\left(e^{-r} \delta \hat{Q}_{1}^{0}-e^{s} \delta \hat{Q}_{2}^{0}\right), & \delta \hat{P}_{B}=\frac{1}{\sqrt{2}}\left(e^{r} \delta \hat{P}_{1}^{0}-e^{-s} \delta \hat{P}_{2}^{0}\right) .
\end{array}
$$


It is easy to check that the same known relations for amplitude difference and phase sum still hold.

$$
\begin{gathered}
\delta \hat{Q}_{+}=\frac{1}{\sqrt{2}}\left(\delta \hat{Q}_{B}+\delta \hat{Q}_{A}\right)=e^{-r} \delta \hat{Q}_{1}^{0} \\
\delta \hat{P}_{-}=\frac{1}{\sqrt{2}}\left(\delta \hat{P}_{B}-\delta \hat{P}_{A}\right)=e^{-s} \delta \hat{P}_{2}^{0}
\end{gathered}
$$

these combinations of quadratures are squeezed in relation to the shot noise level, while the other combinations are anti-squeezed. In the infinite squeezing limit, $r \rightarrow \infty$ and $s \rightarrow \infty$, the analysis done on section (4.2) is retrieved.

Victor's input state does not change.

$$
\begin{gathered}
\hat{Q}_{V}=P_{M}+\delta \hat{Q}_{V}, \\
\hat{P}_{V}=A_{M}+\delta \hat{P}_{V} .
\end{gathered}
$$

When Alice measures the combination of her beam with Victor's, her measurement results are associated with the operators

$$
\begin{gathered}
\hat{Q}_{u}=\frac{1}{\sqrt{2}}\left(P_{M}+\delta \hat{Q}_{V}+\delta \hat{Q}_{A}\right), \\
\hat{P}_{v}=\frac{1}{\sqrt{2}}\left(A_{M}+\delta \hat{P}_{V}-\delta \hat{P}_{A}\right),
\end{gathered}
$$

where $u$ and $v$ are describing different detection schemes measuring different quadratures.

By summing and subtracting Bob's quadratures on the above equation, the state that Bob's beam is project into may be obtained.

$$
\begin{gathered}
\hat{Q}_{B}=P_{M}+\delta \hat{Q}_{V}+\delta \hat{Q}_{A}+\delta \hat{Q}_{B}-\sqrt{2} \hat{Q}_{u}=P_{M}+\delta \hat{Q}_{V}+\sqrt{2} e^{-r} \delta \hat{Q}_{1}^{0}-\sqrt{2} \hat{Q}_{u} \\
\hat{P}_{B}=A_{M}+\delta \hat{P}_{V}+\delta \hat{P}_{B}-\delta \hat{P}_{A}-\sqrt{2} \hat{P}_{v}=A_{M}+\delta \hat{P}_{V}+\sqrt{2} e^{-s} \delta \hat{P}_{2}^{0}-\sqrt{2} \hat{P}_{v}
\end{gathered}
$$

For these calculations, we are not considering the quadrature change on the output of the experiment, which teleports the input quadrature $\hat{P}$ on the output $\hat{Q}$. This correction must be applied on the end of the calculations.

By knowing Bob's quadratures, one knows how to use Alice's measurement information to apply the required transformation.

$$
\begin{gathered}
\hat{Q}_{B} \rightarrow \hat{Q}_{B}+\Gamma \sqrt{2} \hat{Q}_{u}, \\
\hat{P}_{B} \rightarrow \hat{P}_{B}+\Gamma \sqrt{2} \hat{P}_{v} .
\end{gathered}
$$

For perfectly adjusted gains,

$$
\begin{aligned}
& \hat{Q}_{\text {out }}=P_{M}+\delta \hat{Q}_{V}+\sqrt{2} e^{-r} \delta \hat{Q}_{1}^{0}, \\
& \hat{P}_{\text {out }}=A_{M}+\delta \hat{P}_{V}+\sqrt{2} e^{-s} \delta \hat{P}_{2}^{0} .
\end{aligned}
$$

Or if gain is left arbitrary,

$$
\begin{aligned}
& \hat{Q}_{\text {out }}=\quad \Gamma\left(P_{M}+\delta \hat{Q}_{V}\right)+\Gamma \delta \hat{Q}_{A}+\delta \hat{Q}_{B} \\
& =\Gamma \hat{Q}_{V}+\frac{\Gamma}{\sqrt{2}}\left(e^{-r} \delta \hat{Q}_{1}^{0}+e^{s} \delta \hat{Q}_{2}^{0}\right)+\frac{1}{\sqrt{2}}\left(e^{-r} \delta \hat{Q}_{1}^{0}-e^{s} \delta \hat{Q}_{2}^{0}\right) \\
& =\quad \Gamma \hat{Q}_{V}+\frac{\Gamma+1}{\sqrt{2}} e^{-r} \delta \hat{Q}_{1}^{0}+\frac{\Gamma-1}{\sqrt{2}} e^{s} \delta \hat{Q}_{2}^{0} .
\end{aligned}
$$

The analogous work for the other quadrature yields

$$
\hat{P}_{\text {out }}=\Gamma \hat{P}_{V}-\frac{\Gamma+1}{\sqrt{2}} e^{-s} \delta \hat{P}_{2}^{0}-\frac{\Gamma-1}{\sqrt{2}} e^{r} \delta \hat{P}_{1}^{0} .
$$


Eqns (4.57) and (4.58) predict what to expect on an experiment. When $\Gamma=1$, the antisqueezed terms are zero and all that is left is the input state plus the squeezing term. And for $\Gamma \neq 1$, the antisqueezing term becomes more and more important and harms the results.

It is also interesting to notice the classical case inside these equations, i.e. the case where no entangled beams are used. When $r=0, s=0$ and $\Gamma=1$, the output quadratures are Victor's initial quadrature with two additional noise units.

\subsubsection{Detection efficiencies}

The reflections and transmissions suffered by light on the setup, as well as the detector's quantum efficiencies, will degrade the quantum state. For a proper analysis of the results to be expected on a measurement, the losses on every part of the setup must be taken into account [47]. The detectors used on this experiment are InGaAs photodiodes with $0.5 \mathrm{~mm}$ diameter, model IG17X500S4 by Laser Components. It possesses a big reflective characteristic, even with the input window removed the reflection on its surface was around $10 \%$ of the impinging light. To solve this problem, a mirror was placed to direct the reflected beam back into the photodetector. By this feedback method, it was obtained quantum efficiencies up to 0.86 .

Fig (4.9) represents the path taken by light where the suffered losses are represented as beamsplitter transformations. Alice's state is represented by $\left|\alpha_{A}\right\rangle$ on the upper path, while Bob's state is $\left|\alpha_{B}\right\rangle$ on the lower one. Victor's input state is represented by $\left|\alpha_{i n}\right\rangle$. All states represented by $\left|\nu_{z}\right\rangle$ are vacuum states entering the setup by reflection on the beam-splitter $z$, contributing to added noise. The transmission $T_{z}$ and reflection $R_{z}$ of each cube $z$ were measured as

$$
\begin{array}{ll}
T_{A 1}=0.863, & R_{A 1}=0.137, \\
T_{A x}=0.725, & R_{A x}=0.275, \\
T_{B 1}=0.825, & R_{B 1}=0.175, \\
T_{B 2}=0.679, & R_{B 2}=0.321,
\end{array}
$$

where $x$ in $T_{A x}$ and $R_{A x}$ is representative of either $p$ or $q$. These numbers include the detector's quantum efficiency as well as the losses during propagation. They were obtained by measuring the beam's intensity at each point on the setup.

Victor's overall loss $\left(1-T_{B 1} T_{B 2}=0.440\right)$ is greater than Alice's $\left(1-T_{A 1} T_{A x}=0.374\right)$ because the beam reflected on the photodetectors on his station was not sent back to measurement by the feedback method. It was noticed that this method led the beam back through the setup until the OPO chamber, imposing difficulties in locking. Alice's feedback method did not lead to problems, we were able to send the reflected beam back to the detector by an angle which did not led it through the whole setup again. This angle could not be found when dealing with Victor's detectors.

Alice's variance measurement, normalized by shot noise, is

$$
\sigma_{A}^{x}=\frac{1}{2}\left(\Delta^{2} \alpha_{A} T_{A 1}+\Delta^{2} \alpha_{i n}\right) T_{A x}+\frac{R_{A 1} T_{A x}}{2}+R_{A x}
$$

resulting in 1 when considering all terms as shot-noise variances. By performing a measurement with no light, the electronic noise $e^{2}$ due to the detectors can be evaluated and subtracted from the final measurement. However, when Bob receives Alice's information amplifying it by $g_{x}$, this noise will also be amplified by the same gain,

$$
\sigma_{V}^{x}=\Delta^{2} \alpha_{B} T_{B 1} T_{B 2}+g_{x}^{2} \sigma_{A}^{x} T_{B 2}+g_{x} E^{2} T_{B 2}+R_{B 1} T_{B 2}+R_{B 2}
$$

where $E^{2}$ is $e^{2}$ normalized by shot noise. Our measurements yielded

$$
E^{2}=0.0089(1)
$$




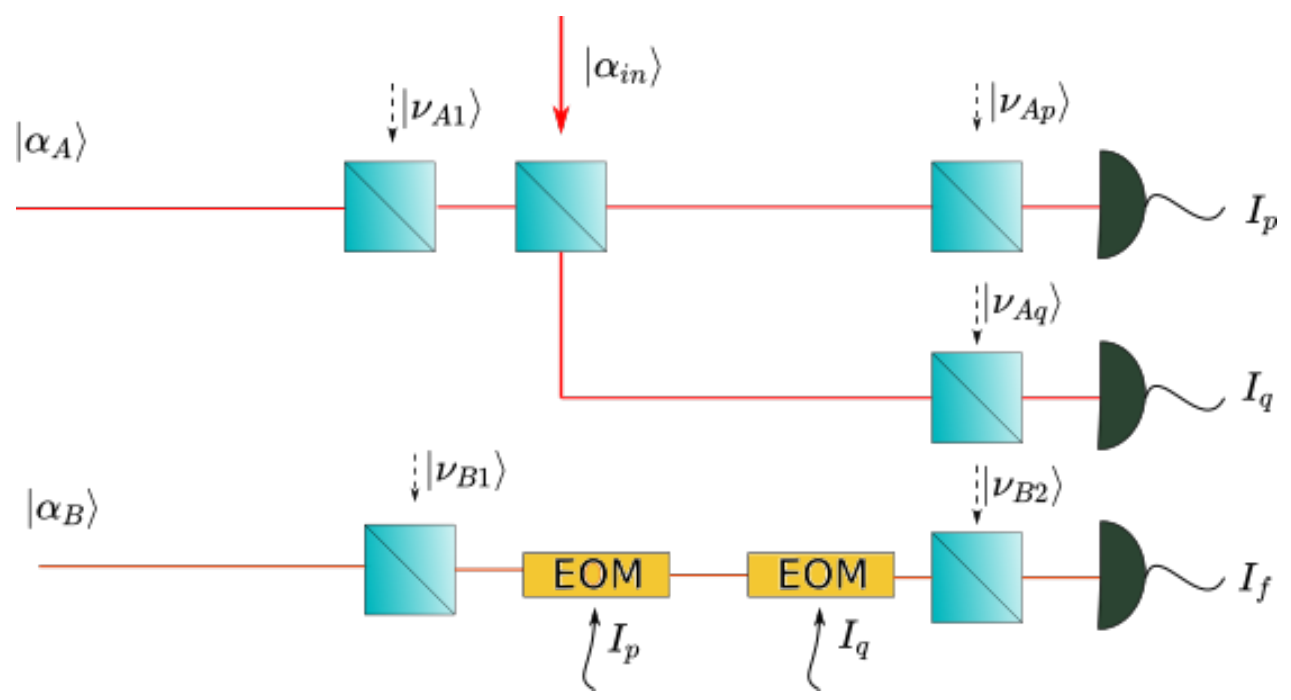

Figure 4.9: Losses modeled as beam-splitter transformations.

Writing the squeezing terms on $\sigma_{V}^{x}$,

$$
\begin{array}{r}
\sigma_{V}^{x}=R_{B 1} T_{B 2}+R_{B 2}+g_{x}^{2}\left(\frac{R_{A 1} T_{A x}}{2}+R_{A x}\right) T_{B 2}+g_{x} E^{2} T_{B 2}+ \\
+\frac{g_{x}^{2}}{2} \Delta^{2} \alpha_{i n} T_{A x} T_{B 2}+ \\
+\frac{e^{-2 r_{-}}}{2}\left(\frac{g_{x}}{\sqrt{2}} t_{A 1} t_{A x}+t_{B 1}\right)^{2} T_{B 2}+\frac{e^{2 r_{+}}}{2}\left(\frac{g_{x}}{\sqrt{2}} t_{A 1} t_{A x}-t_{B 1}\right)^{2} T_{B 2},
\end{array}
$$

where it is written as to contain all spurious vacuum terms on the first line, the input state on the second, and squeezing terms on the last. The quantities $r_{-}$and $r_{+}$are the squeezing and antisqueezing terms, equal to $r$ or $s$ of subsection (4.4.1) depending on the quadrature in question.

It is also interesting to write down the mean values measurements. The state generated by Victor will be measured by Alice with efficiency $T_{A x}$, while Bob will receive this signal amplified by $g_{x}$ and send it to Victor for measurements. It must be reminded that the input state is mixed on a beam-splitter with Alice's state, requiring us to multiply the results of her measurements by $\sqrt{2}$. Bob, however, will receive the state without this correction. The measurements of Alice $-\left\langle\beta_{A}\right\rangle-$ and Victor $-\left\langle\beta_{V}\right\rangle-$ are

$$
\left\langle\beta_{A}\right\rangle=\left\langle\alpha_{i n}\right\rangle t_{A x}, \quad\left\langle\beta_{V}\right\rangle=\frac{g_{x}}{\sqrt{2}}\left\langle\alpha_{i n}\right\rangle t_{A x} t_{B 2}
$$

Since the adjustment of gain $\Gamma$ is done by comparing $\left\langle\beta_{A}\right\rangle$ with $\left\langle\beta_{V}\right\rangle$, we define this parameter as $\Gamma=\frac{g_{x}}{\sqrt{2}} t_{B 2}$, compensating for Victor's detection efficiency. Hence, when $\Gamma=1$, we will have $\left\langle\beta_{A}\right\rangle=\left\langle\beta_{V}\right\rangle$. This definition of $\Gamma$ on the classical case results in Victor's variance measurement of eqn (4.67) being led to

$$
\sigma_{V}^{x}=1+2 \Gamma^{2}+E^{2} \Gamma
$$

We could also consider that we want to retrieve $\left\langle\alpha_{i n}\right\rangle$ on the output instead of $\left\langle\beta_{A}\right\rangle$. Under this view, $T_{A x}$ is a loss on the system, and it is not included on the definition of the input state. In order for us to have $\left\langle\alpha_{i n}\right\rangle=\left\langle\beta_{V}\right\rangle$ for $\Gamma=1$, we need to have $\Gamma=\frac{g_{x}}{\sqrt{2}} t_{A x} t_{B 2}$. Victor's classical variance measurement will then be

$$
\sigma_{V}^{x}=1+2 \frac{\Gamma^{2}}{T_{A x}}+E^{2} \Gamma
$$

leading to a greater variance for every value of $\Gamma$.

In conclusion, the only losses on the system able to harm the obtained results are due to Alice's detection efficiency [47], all other can be compensated by a properly adjusted gain $\Gamma$. 


\subsection{Two-mode teleportation}

When doing a two-mode analysis of the process, extra care must be taken since the Hilbert space is larger. A pure amplitude modulation will create a state on the combinations of quadratures

$$
\hat{p}_{s}=\frac{\hat{p}_{\Omega}+\hat{p}_{-\Omega}}{\sqrt{2}}, \quad \quad \hat{q}_{a}=\frac{\hat{q}_{\Omega}-\hat{q}_{-\Omega}}{\sqrt{2}},
$$

while a pure phase modulation will create a state on the other combinations $\left(\hat{p}_{a}\right.$ and $\left.\hat{q}_{s}\right)$. When we treat asymmetric teleportation, each of these quadratures is independent of each other. Only the modulation parameters $\left(P_{M}\right.$ and $\left.A_{M}\right)$ are not enough to describe the full state of the system. With a doubled Hilbert space, the information is chosen to be organized in a vector. Where

$$
\left|\psi_{i n}\right\rangle=\left(\begin{array}{c}
p_{s}^{i n} \\
q_{a}^{i n} \\
p_{a}^{i n} \\
q_{s}^{i n}
\end{array}\right)
$$

is now the input for teleportation.

\subsubsection{Alice's measurements}

Alice's amplitude measurements are expected to be related to $\hat{p}_{s}$ and $\hat{q}_{a}$ while the phase measurements are expected to be related to $\hat{p}_{a}$ and $\hat{q}_{s}$. Writing in matrix form, the measurements' mean values are related to the input state as

$$
\left(\begin{array}{l}
P_{\mathrm{cos}}^{A} \\
P_{\mathrm{sin}}^{A} \\
Q_{\cos }^{A} \\
Q_{\mathrm{sin}}^{A}
\end{array}\right)=\left(\begin{array}{cccc}
1 & 0 & 0 & 0 \\
0 & 1 & 0 & 0 \\
0 & 0 & \cos \alpha & \sin \alpha \\
0 & 0 & -\sin \alpha & \cos \alpha
\end{array}\right)\left(\begin{array}{c}
p_{s}^{i n} \\
q_{a}^{i n} \\
p_{a}^{i n} \\
q_{s}^{i n}
\end{array}\right)
$$

The measurements of $\hat{p}_{s}$ and $\hat{q}_{a}$ are direct, while $\hat{p}_{a}$ and $\hat{q}_{s}$ are mixed in the photocurrent (subsection (2.8.2) ). The information sent to Bob will again be $P^{A}=P_{\mathrm{cos}}^{A} \cos \Omega t+P_{\sin }^{A} \sin \Omega t$ and $Q^{A}=Q_{\cos }^{A} \cos \Omega t+$ $Q_{\sin }^{A} \sin \Omega t$.

\subsubsection{Victor's measurements}

Victor's mean values measurements are related to Alice's the same way as in equation (4.31). Victor's output state can be related to the input as

$$
\left(\begin{array}{c}
p_{s}^{V} \\
q_{a}^{V} \\
p_{a}^{V} \\
q_{s}^{V}
\end{array}\right)=\left(\begin{array}{cccc}
0 & 0 & -\sin (\alpha+\beta) & \cos (\alpha+\beta) \\
0 & 0 & -\cos (\alpha+\beta) & -\sin (\alpha+\beta) \\
1 & 0 & 0 & 0 \\
0 & 1 & 0 & 0
\end{array}\right)\left(\begin{array}{c}
p_{s}^{i n} \\
q_{a}^{i n} \\
p_{a}^{i n} \\
q_{s}^{i n}
\end{array}\right)
$$

and when the gains are not perfectly adjusted

$$
\left(\begin{array}{l}
p_{s}^{V} \\
q_{a}^{V} \\
p_{a}^{V} \\
q_{s}^{V}
\end{array}\right)=\left(\begin{array}{cccc}
-g_{p} \sin \phi_{p} & 0 & -g_{q} \cos \phi_{q} \sin (\alpha+\beta) & g_{q} \cos \phi_{q} \cos (\alpha+\beta) \\
0 & -g_{p} \sin \phi_{p} & -g_{q} \cos \phi_{q} \cos (\alpha+\beta) & -g_{q} \cos \phi_{q} \sin (\alpha+\beta) \\
g_{p} \cos \phi_{p} & 0 & -g_{q} \sin \phi_{q} \sin (\alpha+\beta) & g_{q} \sin \phi_{q} \cos (\alpha+\beta) \\
0 & g_{p} \cos \phi_{p} & -g_{q} \sin \phi_{q} \cos (\alpha+\beta) & -g_{q} \sin \phi_{q} \sin (\alpha+\beta)
\end{array}\right)\left(\begin{array}{l}
p_{s}^{i n} \\
q_{a}^{i n} \\
p_{a}^{i n} \\
q_{s}^{i n}
\end{array}\right) .
$$

The two equations above are the the most general form of the teleportation equations. When analysing measurements, we must use them to understand how the input quadratures will be presented on Victor's measurements. The output state (indice out) is obtained by the application of the inverse of the above matrix on Victor's quadrature vector (indice $V$ ). If teleportation is successful, $\hat{x}^{\text {out }}=\hat{x}^{\text {in }}$ where $\hat{x}$ represents any of the four possible quadratures. 


\subsection{Fidelity}

The most common figure of merit for checking the quality of a teleportation process is the fidelity $\mathcal{F}$ 46]. When the input state is pure, the most general definition is

$$
\mathcal{F}=\left\langle\psi_{\text {in }}\left|\hat{\rho}_{\text {out }}\right| \psi_{\text {in }}\right\rangle
$$

where $\psi_{\text {in }}$ is the input state and $\hat{\rho}_{\text {out }}$ is the density matrix describing the output state. On this experiment on continuous variables, the teleported states are coherent $\left|\psi_{\text {in }}\right\rangle=\left|\alpha_{\text {in }}\right\rangle$ and $\hat{\rho}_{\text {out }}=\left|\alpha_{\text {out }}\right\rangle\left\langle\alpha_{\text {out }}\right|$ (section (2.2)), hence

$$
\mathcal{F}=\left|\left\langle\alpha_{\text {in }} \mid \alpha_{\text {out }}\right\rangle\right|^{2}=\operatorname{Tr}\left[\hat{\rho}_{\text {in }} \hat{\rho}_{\text {out }}\right]
$$

where $\hat{\rho}_{i n}=\left|\alpha_{i n}\right\rangle\left\langle\alpha_{i n}\right|$. This can be calculated by making use of Wigner functions [21]. According to eqn. (2.39),

$$
\mathcal{F}=\pi^{n} \int_{C^{n}} d \mathbf{x} W\left[\hat{\rho}_{\text {in }}\right](\mathbf{x}) W\left[\hat{\rho}_{\text {out }}\right](\mathbf{x}) .
$$

If we first consider the case where the input state is symmetric on sidebands' energy, that can be described as just one coherent state, the equation above is a two variable gaussian integral. Since this is a gaussian state following the hypothesis of section (2.4), the Wigner function is eqn (2.37), which leads to the fidelity

$$
\mathcal{F}=\frac{2}{\sqrt{(1+\alpha)(1+\beta)}} \exp \left[-\frac{1}{2}\left(\frac{\left(P_{M}^{\text {out }}-P_{M}^{\text {in }}\right)^{2}}{1+\alpha}+\frac{\left(A_{M}^{\text {out }}-A_{M}^{\text {in }}\right)^{2}}{1+\beta}\right)\right]
$$

where $\alpha$ and $\beta$ are respectively the variances of the output quadratures $\hat{P}$ (the quadrature where Victor measures $P_{M}$ ) and $\hat{Q}$ (where $A_{M}$ is measured). For coherent input states, variances equal 1 . The superscript in and out refer to the input - measured by Alice on eqns (4.18) through (4.21) - and to the output - measured by Victor on eqn (4.32) — states. From the photocurrents, $P_{M}^{i n}$ can be retrieved by $\sqrt{\left(Q_{\cos }^{A}\right)^{2}+\left(Q_{\sin }^{A}\right)^{2}}$ and other modulations can be calculated analogously.

If, instead, Victor decides to use his cavity to teleport an asymmetric state on the sidebands, the input state is now

$$
\left|\psi_{\text {in }}\right\rangle=\left|\alpha_{-\Omega}\right\rangle \otimes\left|\alpha_{\Omega}\right\rangle
$$

with each sideband being a different coherent state, $\left|\alpha_{-\Omega}\right\rangle$ and $\left|\alpha_{\Omega}\right\rangle$. It is assumed that $\delta=0$ and $\gamma=0$ on the covariance matrix, implying same noise power on the quadratures and aligned noise ellipse. This yields the equation [16]

$$
\mathcal{F}_{2}=\frac{4}{(1+\alpha)(1+\beta)} \exp \left[-\frac{1}{2}\left(\frac{\left(\bar{p}_{s}^{\text {out }}-\bar{p}_{s}^{\text {in }}\right)^{2}+\left(\bar{q}_{a}^{\text {out }}-\bar{q}_{a}^{\text {in }}\right)^{2}}{1+\alpha}+\frac{\left(\bar{q}_{s}^{\text {out }}-\bar{q}_{s}^{\text {in }}\right)^{2}+\left(\bar{p}_{a}^{\text {out }}-\bar{p}_{a}^{\text {in }}\right)^{2}}{1+\beta}\right)\right]
$$

where in and out refer to the input and output states as in eqn (4.78). The overline over the quadratures' values represents the calculation of a mean value. This equation has a simple interpretation, it is the multiplication of two one-mode fidelities: one considering only the symmetric combination of quadratures, and the other considering only the antisymmetric combination.

$$
\begin{aligned}
& \mathcal{F}_{s}=\frac{2}{\sqrt{(1+\alpha)(1+\beta)}} \exp \left[-\frac{1}{2}\left(\frac{\left(\bar{p}_{s}^{\text {out }}-\bar{p}_{s}^{\text {in }}\right)^{2}}{1+\alpha}+\frac{\left(\bar{q}_{s}^{\text {out }}-\bar{q}_{s}^{\text {in }}\right)^{2}}{1+\beta}\right)\right] \\
& \mathcal{F}_{a}=\frac{2}{\sqrt{(1+\alpha)(1+\beta)}} \exp \left[-\frac{1}{2}\left(\frac{\left(\bar{q}_{a}^{\text {out }}-\bar{q}_{a}^{\text {in }}\right)^{2}}{1+\alpha}+\frac{\left(\bar{p}_{a}^{\text {out }}-\bar{p}_{a}^{\text {in }}\right)^{2}}{1+\beta}\right)\right] .
\end{aligned}
$$

\subsubsection{Benchmarks}

A teleportation scheme in continuous variables should teleport the whole state of the system. For this reason, it may seem odd that checking only two non commuting observables like $\hat{P}$ and $\hat{Q}$ are 
enough for evaluating success of the protocol. However, it is possible to show that this is enough to claim that any statistics for an arbitrary observable that would be measured at Victor's input state are also valid for the final output state.

If the probability density for the measurement of an observable $\hat{O}_{n}$, with measurement outcomes $n$, for the input and output states are

$$
\begin{gathered}
P_{\text {in }}(n)=\operatorname{Tr}\left(\hat{\rho}_{\text {in }} \hat{O}_{n}\right), \\
P_{\text {out }}(n)=\operatorname{Tr}\left(\hat{\rho}_{\text {out }} \hat{O}_{n}\right),
\end{gathered}
$$

the overlap of these probabilities is

$$
\left.\int d n \sqrt{P_{\text {in }}(n) P_{\text {out }}(n)} \geq \operatorname{Tr}\left[\hat{\rho}_{\text {in }} \hat{\rho}_{\text {out }}\right)\right]=\mathcal{F},
$$

regardless of the observable, showing that fidelity is indeed a good parameter for analysing teleportation. It acts as a lower limit on the comparison of probality densities for any obervable for the two states [48].

Classical teleportation is limited by an upper boundary on the fidelity measurement, one that can only be overcome by quantum teleportation. Eqn (4.30) for the classical case with fine adjusted gains is

$$
\hat{a}_{\text {out }}=\alpha_{B}+\frac{1}{2}\left[\delta \hat{Q}_{V}+P_{M}+\delta \hat{Q}_{A}+\delta \hat{Q}_{B}+i\left(\delta \hat{P}_{V}+A_{M}-\delta \hat{P}_{A}+\delta \hat{P}_{B}\right)\right] .
$$

This is to be compared with the input (eqn (4.14))

$$
\hat{a}_{i n}=\alpha_{V}+\frac{1}{2}\left[\delta \hat{P}_{V}+A_{M}+i\left(\delta \hat{Q}_{V}+P_{M}\right)\right]
$$

The noises of each party (Alice, Bob and Victor) cannot cancel each other out because they are not correlated. Each of these states are coherent, so all their quadratures noise are shot noise level (eqn (2.22) ) $\left(\alpha_{\text {in }}=1\right.$ and $\left.\beta_{\text {in }}=1\right)$. For $\alpha_{\text {out }}$ and $\beta_{\text {out }}$, we must sum three normalized coherent states' noises as seen in eqn (4.86). The result, in shot noise units, is

$$
\begin{aligned}
\alpha_{\text {out }} & =\left(\delta \hat{Q}_{V}+\delta \hat{Q}_{A}+\delta \hat{Q}_{B}\right)^{2}=3, \\
\beta_{\text {out }} & =\left(\delta \hat{P}_{V}-\delta \hat{P}_{A}+\delta \hat{P}_{B}\right)^{2}=3
\end{aligned}
$$

By plugging this result into eqn (4.78), we have

$$
\mathcal{F}_{\text {class }}=\frac{1}{2}
$$

giving us the maximum fidelity possible to be achieved in classical teleportation. We have $\bar{P}_{\text {out }}=\bar{P}_{\text {in }}$ and $\bar{Q}_{\text {out }}=\bar{Q}_{\text {in }}$ - input and output quadratures are the same - but nothing better can be done for the variances. By the same logic, it is easy to see that eqn (4.80) works as a product of two fidelity equations, one for the symmetric combinations of quadratures and another one for the antisymmetric. The benchmark in this case is $\mathcal{F}_{\text {class }}=(1 / 2) *(1 / 2)=\frac{1}{4}$.

Overcoming the $1 / 2$ benchmark guarantees that entanglement was used in the process. However, there is another benchmark that should be taken into account, the no cloning limit [49]. This limit is achieved by considering that the gathered information about the state is sent to $M$ parties so they can reconstruct Victor's state at their station. The maximum fidelity one can achieve is

$$
\mathcal{F}_{M} \leq \frac{M}{2 M-1}
$$

If the information is used to construct an infinite number of copies of the initial state, we have $M \rightarrow \infty$ and $\mathcal{F}_{\infty} \leq \frac{1}{2}$, which is the classical limit. This makes sense because, in the classical case, Alice's information of measurement can be used by anyone to reconstruct Victor's state, since there is no need of correlated fields. 
However, if we consider a teleportation case in which Alice did not destroy the original state at the input and sent only part of the information she obtained to Bob, we will have two existing copies of the state, $M=2$. This implies $\mathcal{F}_{2} \leq \frac{2}{3}$ for each existing copy. Overcoming this benchmark means that the best remaining copy of the initial state is in Bob's possession. For the two-mode teleportation, the benchmark is squared $\mathcal{F}_{\text {two-mode }}=4 / 9$.

At last, in the case where there is only one remaining copy of the original state, we have $M=1$ and $\mathcal{F}_{1} \leq 1$. This is the case of teleportation using entanglement and no extra copies.

These benchmarks can be visualized by some calculations done on eqn (4.78). For perfect teleportation, we must have $\alpha_{\text {out }}=1$ and $\beta_{\text {out }}=1$. By plugging this result on the equation we arrive at the expected $\mathcal{F}=1$. For classical teleportation, where $\alpha_{\text {out }}=3$ and $\beta_{\text {out }}=3$, we have $\mathcal{F}=\frac{1}{2}$. For the no cloning limit, we may consider that Alice splits Victor's beam in two, making measurements in one of the beams and keeping the other to herself. The beam splitter transformation adds one shot noise unit to the state, so Bob's final state can be no better than $\alpha_{\text {out }}=2$ and $\beta_{\text {out }}=2$, which leads to $\mathcal{F}=\frac{2}{3}$. For the sake of the argument, it was considered that $\bar{P}_{\text {out }}=\bar{P}_{\text {in }}$ and $\bar{Q}_{\text {out }}=\bar{Q}_{\text {in }}$.

\subsubsection{Losses}

The measurement of three units of shot noise on Victor's station was predicted by eqn (4.68), when a comparison between Alice's measument and Victor's measurement was made. However, teleportation is performed by comparing the input state generated by Victor with the measured output state. The variances are then expected to behave as eqn (4.69), which, under the losses presented on our system, results in 3.759 units of shot noise at the output. Calculating fidelity yields a classical limit of $\mathcal{F}=0.420$, leading to a two mode fidelity limit of $\mathcal{F}=0.176$.

The state generated by Victor could also be compared with the state generated by Bob, before measurement. For this, we need to take eqn (4.69) uncorrected by Victor's detection efficiency $T_{B 2}$. Bob's variance is obtained from Victor's by the transformation

$$
\begin{gathered}
\sigma_{V}^{x}=\sigma_{B}^{x} T_{B 2}+R_{B 2}, \\
\Rightarrow \sigma_{B}^{x}=1+2 \frac{\Gamma^{2}}{T_{A x} T_{B 2}}+E \Gamma,
\end{gathered}
$$

yielding $\sigma_{B}^{x}=5.063$, which leads to the classical limit of $\mathcal{F}=0.330$. The numbers are far from ideal because the experiment was not optimized to perform under this interpretation of input and output state.

When the analysis is to be presented, the obtained results will be calculated by comparison to the output Victor's state of the input by considering the losses as part of it (limit of $\mathcal{F}=0.5$ ), and by not considering it (limit of $\mathcal{F}=0.420$ ). The former will be called the uncorrected state from now on, while the latter will be called the corrected state.

The fidelity results for the uncorrected input state are greater than for the corrected one, because we are considering that Alice measures the input state perfectly, with no losses added. By considering the imperfect detection efficiencies, the input state can be corrected for the real input state for the teleportation, the one Victor created and that will result in a fidelity no better that $\mathcal{F}=0.420$. 


\subsection{Expected results}

Subsection (4.4.1) lead us to eqns (4.57) and (4.58) that allows us to write the output states as a function of the gain.

$$
\begin{array}{cc}
\bar{P}_{\text {out }}= & \Gamma \bar{P}_{\text {in }}, \\
\bar{Q}_{\text {out }}= & \Gamma \bar{Q}_{\text {in }}, \\
\alpha_{\text {out }}= & \Gamma^{2}+\frac{(\Gamma+1)^{2}}{2} e^{-2 s}+\frac{(\Gamma-1)^{2}}{2} e^{2 r}, \\
\beta_{\text {out }}= & \Gamma^{2}+\frac{(\Gamma+1)}{2} e^{-2 r}+\frac{(\Gamma-1)^{2}}{2} e^{2 s} .
\end{array}
$$

The variances can be corrected for a more realistic scenario by using the results of subsection (4.4.2). If we are comparing the uncorrected input state with Victor's measurements, then we define $\Gamma=\frac{g_{x}}{\sqrt{2}} t_{B 2}$ and we are lead to

$$
\begin{aligned}
& \sigma_{V}^{x}=R_{B 1} T_{B 2}+R_{B 2}+\Gamma^{2}\left(R_{A 1} T_{A x}+R_{A x}+1+E^{2}\right)+ \\
& +\frac{e^{-2 r_{-}}}{2}\left(\Gamma t_{A 1} t_{A x}+t_{B 1} t_{B 2}\right)^{2}+\frac{e^{2 r_{+}}}{2}\left(\Gamma t_{A 1} t_{A x}-t_{B 1} t_{B 2}\right),
\end{aligned}
$$

followed from eqn (4.67). Nomenclature is the same used on the referred subsection. The above equation is valid for $x=p$ or $q$ (making $\sigma_{V}^{x}$ representative of $\alpha_{\text {out }}$ or $\beta_{\text {out }}$ ), where the correct squeezing parameters must be written.

If the input state is corrected by the losses, Alice's detection efficiencies will harm the results. We must define $\Gamma=\frac{g_{x}}{\sqrt{2}} t_{A x} t_{B 2}$ and we are led to

$$
\begin{aligned}
\sigma_{V}^{x}= & R_{B 1} T_{B 2}+R_{B 2}+\Gamma^{2}\left(R_{A 1}+2 \frac{R_{A x}}{T_{A x}}+1+E^{2}\right)+ \\
& +\frac{e^{-2 r_{-}}}{2}\left(\Gamma t_{A 1}+t_{B 1} t_{B 2}\right)^{2}+\frac{e^{2 r_{+}}}{2}\left(\Gamma t_{A 1}-t_{B 1} t_{B 2}\right) .
\end{aligned}
$$

So far, fidelity was only analysed for perfectly adjusted gains. The above discussion allows us to rewrite eqn (4.78) as a function of $\Gamma$, telling us what to expect when the gains are not ideal,

$$
\mathcal{F}=\frac{2}{\sqrt{\left(1+\alpha_{\text {out }}\right)\left(1+\beta_{\text {out }}\right)}} \exp \left[-\frac{1}{2}(\Gamma-1)^{2}\left(\frac{\bar{P}_{\text {in }}^{2}}{1+\alpha_{\text {out }}}+\frac{\bar{Q}_{\text {in }}^{2}}{1+\beta_{\text {out }}}\right)\right] .
$$

$\alpha_{\text {out }}$ and $\beta_{\text {out }}$ are also functions of $\Gamma$, but are not explicitly written because different comparisons will result on different definitions, as seen above.

On the experimental setup, gain $\Gamma$ is controlled by manipulating the power of the laser. Since the input quadratures' mean values $\bar{P}_{i n}$ and $\bar{Q}_{i n}$ are generated by taking some part of the carrier's amplitude as seen on subsection (2.7), they are related to the gain in the following manner

$$
\begin{gathered}
\bar{P}_{i n}=-\sqrt{\Gamma} M_{p}, \\
\bar{Q}_{i n}=\sqrt{\Gamma} M_{q},
\end{gathered}
$$

where $M_{p}$ and $M_{q}$ are related to the electronic modulation (eqns (2.79) and (2.80) using the approximation $\left.\left|\alpha_{A}\right| \approx\left|\alpha_{B}\right|=\sqrt{\Gamma}\right)$. We can use this information to arrive at a more realistic fidelity equation,

$$
\mathcal{F}=\frac{2}{\sqrt{\left(1+\alpha_{\text {out }}\right)\left(1+\beta_{\text {out }}\right)}} \exp \left[-\frac{\Gamma}{2}(\Gamma-1)^{2}\left(\frac{M_{p}^{2}}{1+\alpha_{\text {out }}}+\frac{M_{q}^{2}}{1+\beta_{\text {out }}}\right)\right] .
$$

\subsubsection{Classical teleportation}

Fig (4.10) is a plot of the fidelity as a function of (real part of) $\Gamma$ for the classical case under eqn (4.100), considering the uncorrected input state. Each curve represents a different $\xi_{i n}=\sqrt{\bar{P}_{i n}^{2}+\bar{Q}_{i n}^{2}}$, 


\section{Fidelity}

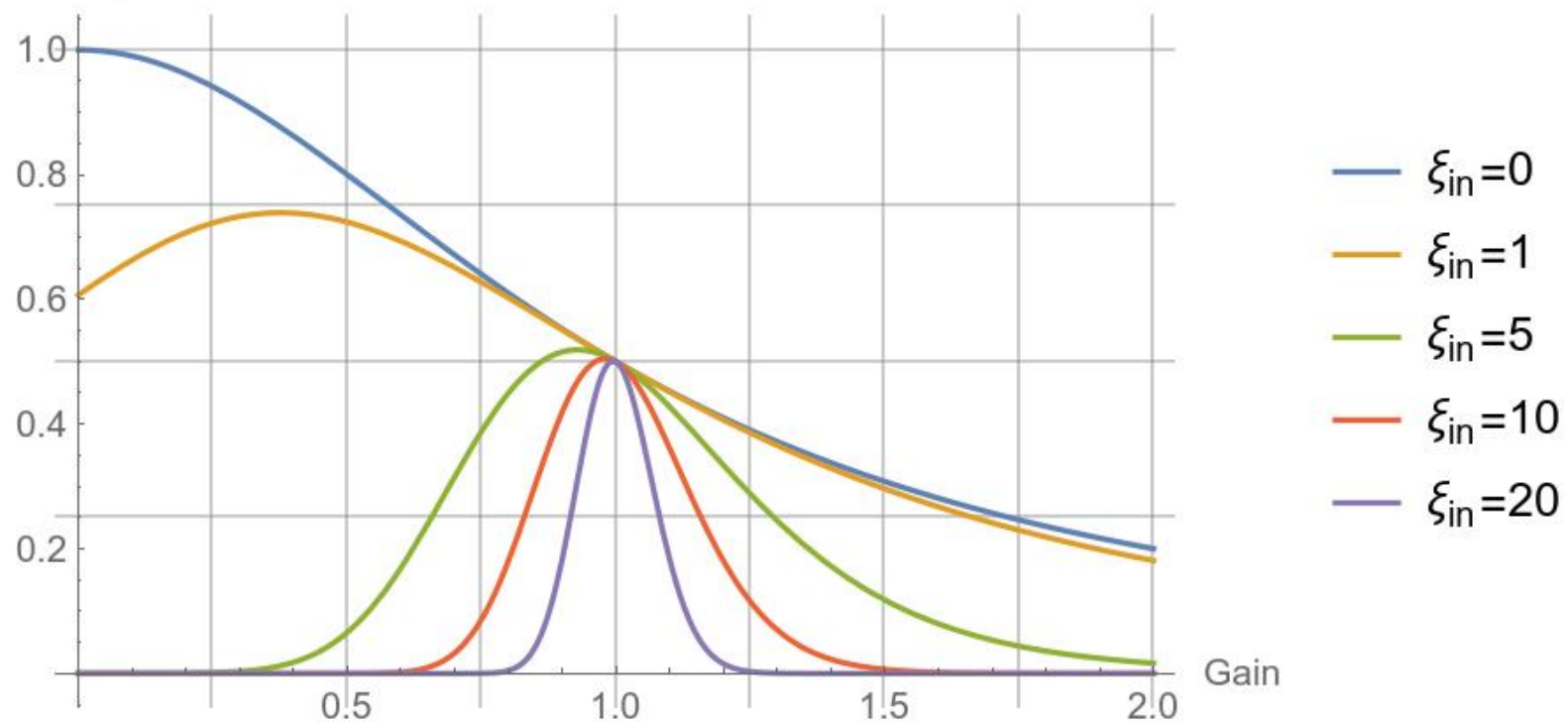

Figure 4.10: Fidelity as a function of $\Gamma$ for different input states.

the amplitude of the state. Some regions of the graph exhibit a fidelity greater than the $\frac{1}{2}$ established classical limit. This happens for smaller states (small $\xi_{\text {in }}$ ) and for $\Gamma<1$. To explain why this happens, consider the case $\xi_{\text {in }}=0$, called vacuum teleportation. $\Gamma=0$ represents a scenario where Alice doesn't send any information to Bob. Nonetheless, since his initial state is already a state with zero mean value and shot noise variance - exactly as the initial vacuum state - , calculating fidelity would result in $\mathcal{F}=1$. However, this cannot be called teleportation, because Alice is using prior information about Victor's input state to decide not to inform Bob about her measurement. Only for $\Gamma=1$ it is guaranteed that a truly unknown state is being teleported, making it the point where the $\frac{1}{2}$ benchmark is valid [50].

Fidelity

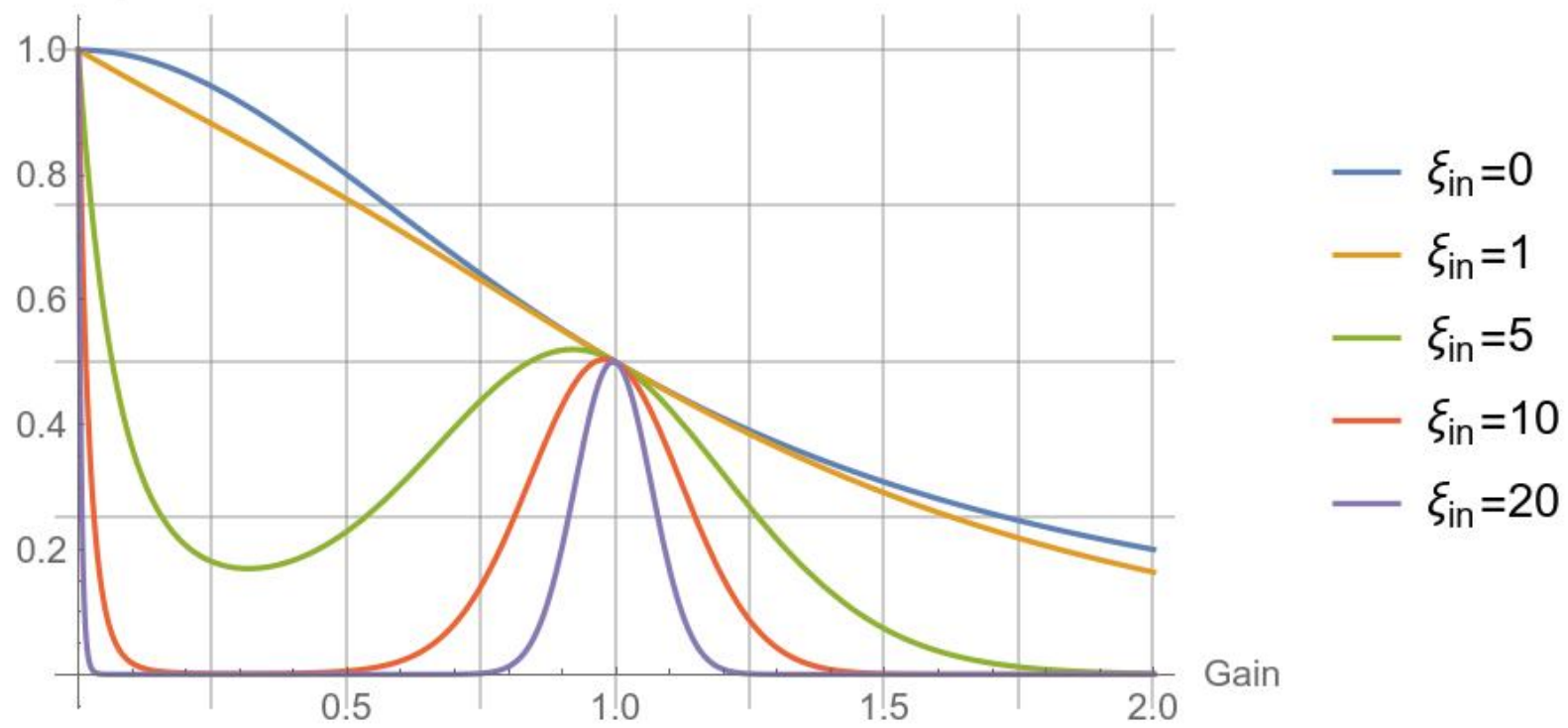

Figure 4.11: More realistic simulation for the equation of fidelity.

The more realistic eqn (4.103) replaces fig (4.10) for fig (4.11). Now $\mathcal{F}(\Gamma=0)=1$ for every input state because at zero gain the input is always a vacuum state. The results from fig (4.10) are retrieved as $\Gamma$ approaches 1 and is greater than it. 
Fidelity



No losses

Losses

Figure 4.12: Comparison between expect results around $\Gamma=1$ for the uncorrected input state (in blue, no losses being corrected from the measurements) and for the corrected one (in yellow, losses corrected from the measurements).

When we consider the losses on the input state, Alice's detection efficiency will harm the state. Fig (4.12) compares the prediction between both possible definitions of input on the system. A state with $\xi_{\text {in }}=20$ was chosen to be shown around $\Gamma=1$ region. The fidelity limit is now $\mathcal{F}=0.420$. 


\subsubsection{Quantum teleportation}

$s=r=0.3$
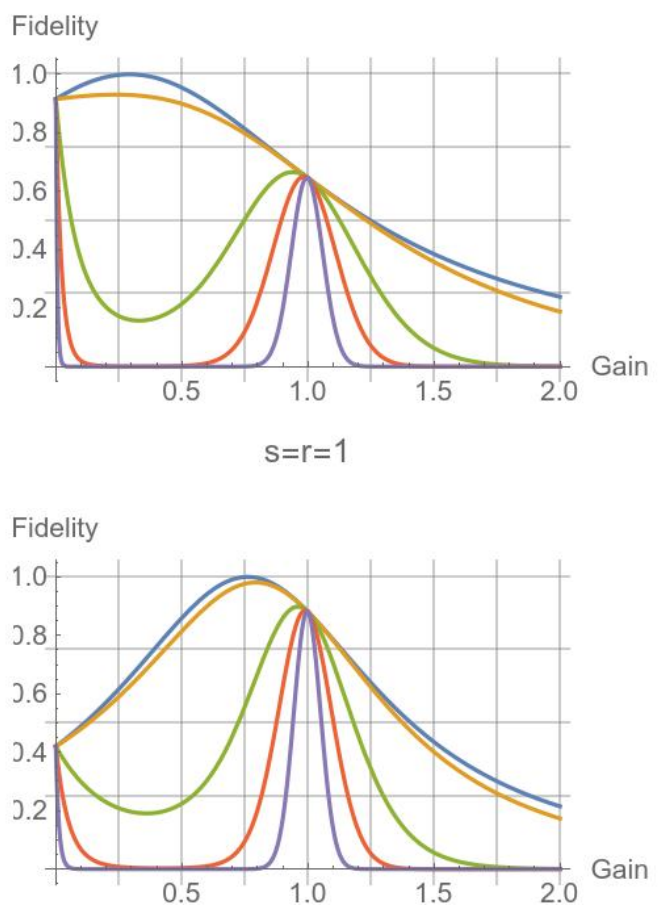

$\mathrm{s}=\mathrm{r}=0.5$
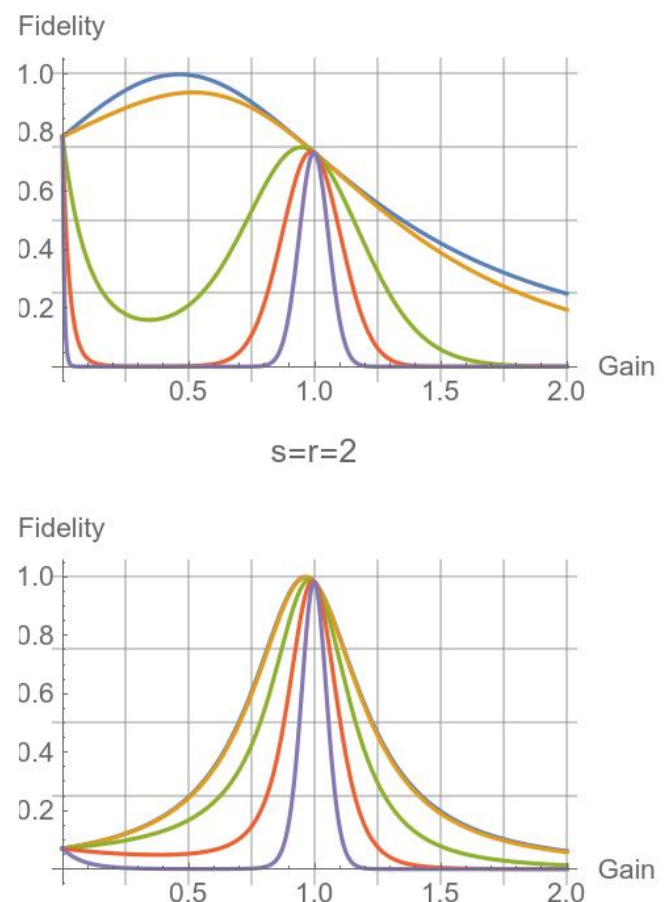

Figure 4.13: Fidelity graphic for different squeezing parameters. Colors indicate input states as fig (4.10).

Fig (4.13) exhibits expected results for quantum teleportation considering different squeezing parameters for the uncorrected input state. As squeezing increases, fidelity approaches 1 for unitary gain. Furthermore, the peak of maximum fidelity for each $\xi_{\text {in }}$ is approaching $\Gamma=1$. On the quantum case, it is not true that $\mathcal{F}=1$ for $\Gamma=0$ because, for entangled states, the noise of each quadrature for each individual beam is greater than shot noise and it is even greater as squeezing increases, so Bob's initial state is a thermal state and not coherent vacuum.

On this experiment, fidelity is expected to behave as shown on fig (4.14), where $\mathcal{F}=0.598$ is predicted to be measured at unity gain. If the input state is corrected by the losses, the graphs will have similar behaviour but with $\mathcal{F}=0.500$ for $\Gamma=1$, above the classical limit of 0.420 for this case. Under any interpretation, it is expected to achieve improvements in relation to the classical setup. The squeezing parameters used for the plot are the ones obtained from fig (3.9) on subsection (3.2.5).

All calculations presented here were done considering a single-mode teleportation. Extension to two-mode is done by squaring all the results. 
Fidelity

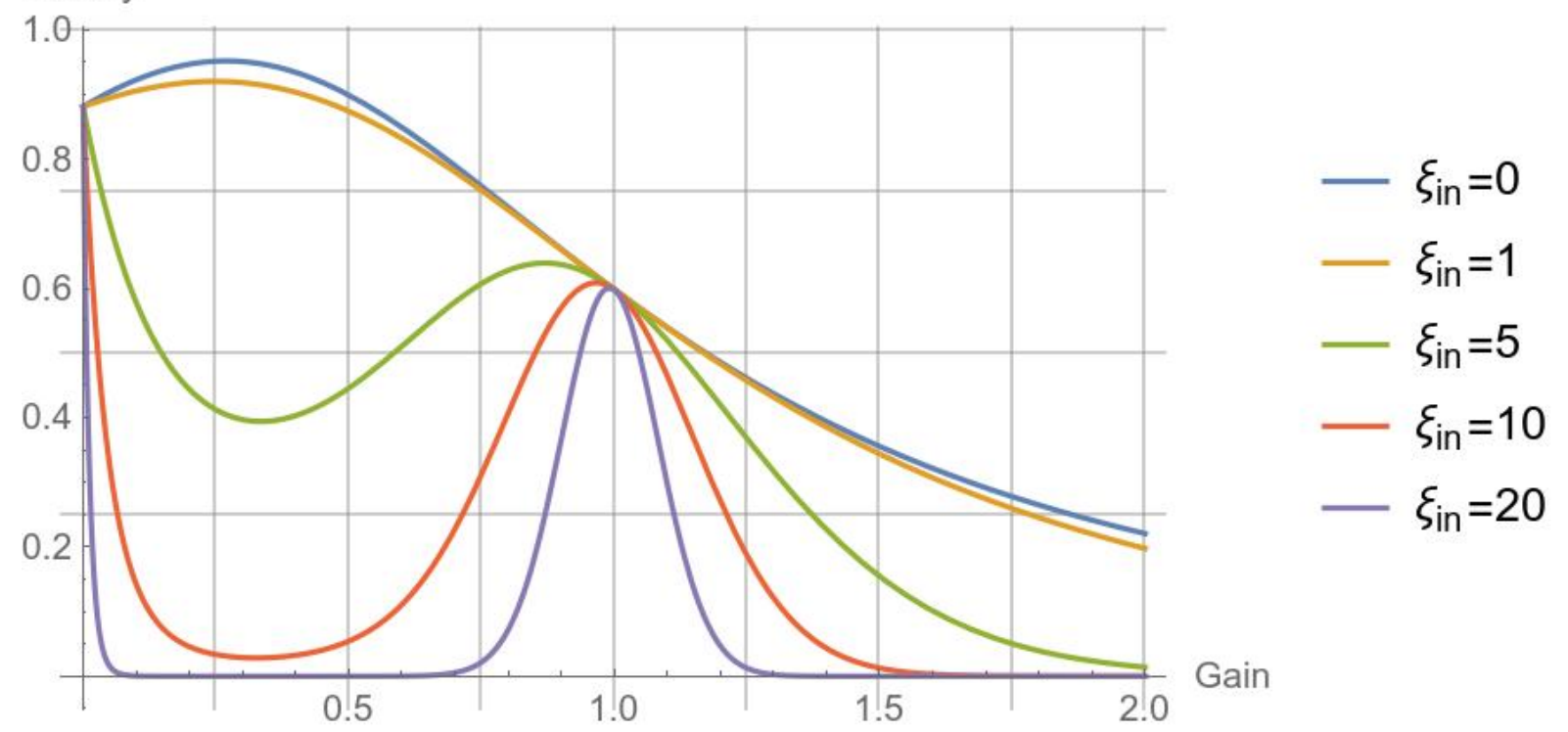

Figure 4.14: Expected fidelity for squeezing parameters obtained from fits. 


\section{EXPERIMENTAL RESULTS}

From last chapter, it is known that, depending on the resource, we may perform classical or quantum teleportation, each with the possibility of being described by one-mode analysis or two-mode analysis. Mean values and variances of quadratures are measured for different intensities, enabling us to study the output of teleportation for different gain conditions. Besides, the analysis can be done for the uncorrected or corrected input state.

Fig (4.2) shows four detectors for sideband measurement: two at Alice's station and two at final Victor's station. Each one separates the $D C$ part of the signal - the zero frequency carrier information — from the $H F$ — which includes the $15 \mathrm{MHz}$ sideband information. The latter contains the desired information we want to analyse, requiring a demodulation chain, as explained in section (2.6), for the photocurrent to result on the measurements described on sections (4.2) and (4.5).

However, before applying the theory of last chapter to the measurements, two treatments must be done to the raw data. First, since the different electronics of different detectors will introduce different amounts of noise, the relative gain between detectors must be calculated so measurements can be compared. And second, shot noise is to be measured and used as a normalization. Only after that, the data can be used for analysis of teleportation. We can separate this treatment on three steps: the first is gain adjustment - this is the gain that differs from each detection scheme, not the gain of Alice's information sent to Bob — , the second is shot noise normalization and the last one is the final teleportation analysis. This is the theme of section (5.1). The following sections show the obtained results on the experiment after raw data is treated. First, classical teleportation is presented (section $(5.2))$ and finally quantum measurements are shown (section (5.3)).

\subsection{Analysis steps}

The first two steps are performed with measurements of light without modulation and are used as a calibration for the third step. To get rid of electronic noises, we also must take one measurement with no light, the so called dark, and subtract it from every data, with the exception of data used for gain adjustment. The analysis is performed on a Wolfram Mathematica notebook written for this purpose.

\subsubsection{Gain adjustment}

The $1064 \mathrm{~nm}$ laser beam from Diabolo is equally divided into the four detectors by comparing the $D C$ signal. Measurements are taken at different intensities without added modulations, resulting in shot noise variances around a zero mean value for the sideband's quadratures. Since the interest is only on the detector's electronics, there is no need for cavity locking. Electronic dark noise is not subtracted at this stage, since it will not affect the gain.

With the data at hand, the standard deviation of the $H F$ signal of the cosine demodulation of one of Alice's detector is plotted against the other detector on her station and fitted to a straight line. The same procedure is performed for the sine part and for Victor's cosine and sine signals, but plots should not mix different demodulations or parties because we want to compare detectors measuring the same state.

Fig (5.1) shows one result as an example. The angular coefficient of the fitting is taken as the desired gain — the proportionality between measurements of different detectors. This factor must be corrected on all subsequent measurements of the day in order to make comparisons between results of different detectors possible. 
VictorCos
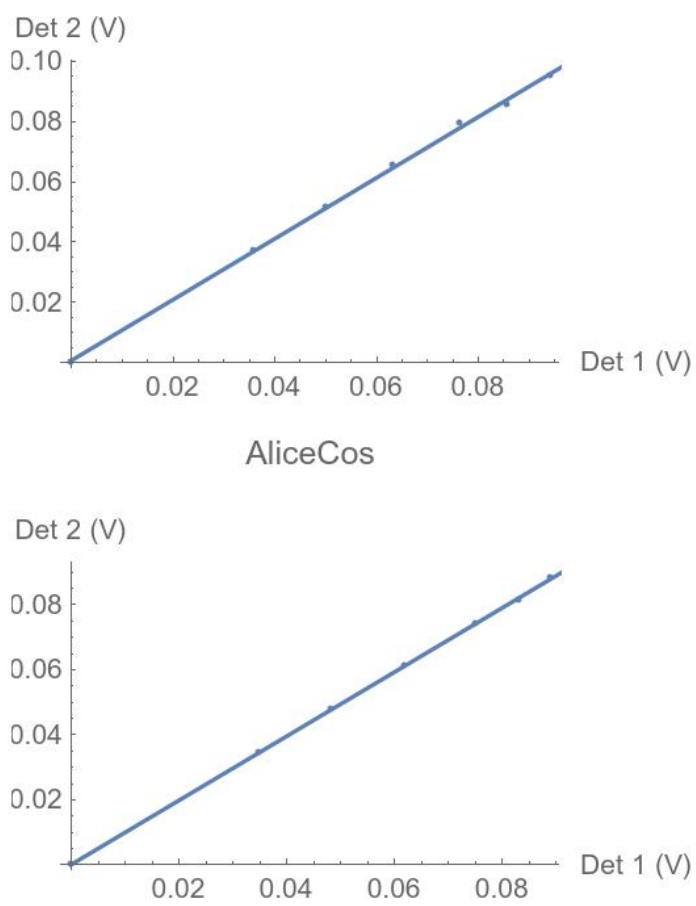

VictorSin
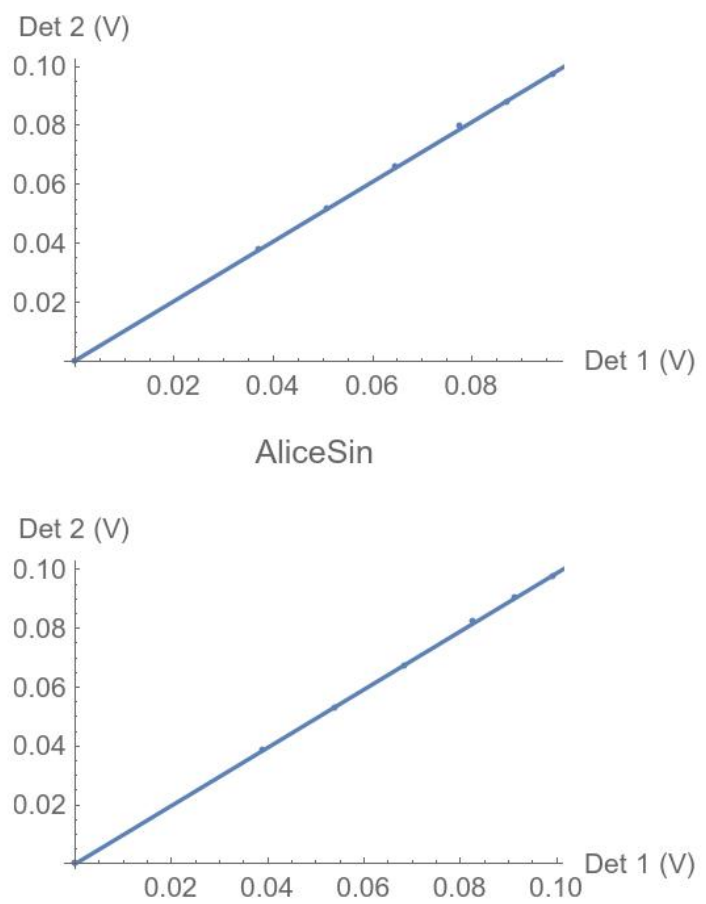

Figure 5.1: Example of one measurement for gain adjustment. The standard deviation measures of two states are plotted against each other. All data is in units of volts as measured by the photodetectors.

\subsubsection{Shot noise normalization}

The same data used for gain adjustment can be used for shot noise normalization, but adjusted by the gain factor calculated on the first step. The sum of intensities of the detectors on each station is plotted against the variance of the sum of the measured fluctuations, as well as against the variance of its subtraction. We are using both detectors on the same station for each graph, as seen on fig (5.2).

If the beam is perfectly coherent, there is no difference between the variances of the sum and of the subtraction of the $H F$ signal, since each detector will measure uncorrelated vacuum noise. The greater value for the variance of the sum shown on the graphs is caused by excess noise present on the laser source.

The fitted curve on the figure takes into account only the subtraction of the fluctuations as a function of the $D C$ signal, getting rid of excess noise. The variance of the sum is shown on graphs only for comparison. The $H F$ part of eqn (2.49) leads us to

$$
I_{H F}^{2}(t)=|\alpha|^{2}\left(e^{i 2 \theta} \Delta^{2} \hat{a}^{\dagger}+e^{-i 2 \theta} \Delta^{2} \hat{a}\right),
$$

making explicit the linear relation between shot noise and light's intensity. The fitted equation is $y=k * x$ where $x$ is the beam's intensity and $k$ is a free parameter related to the shot noise values.

The obtained linear coefficient is used as calibration for all the measurements performed on the day. The variances of the $H F$ signal that will be presented here are corrected by this values while the mean values are corrected by the square root of it. It is said that the variances are measured in shot noise units and the mean values in square root of shot noise units, normalization used for the remaining of the text. 
VictorCos

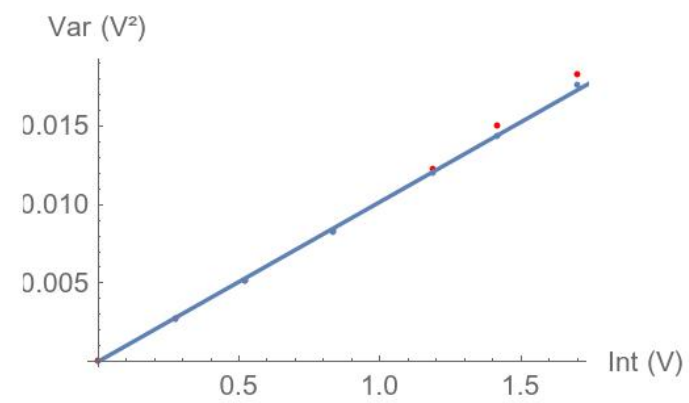

AliceCos

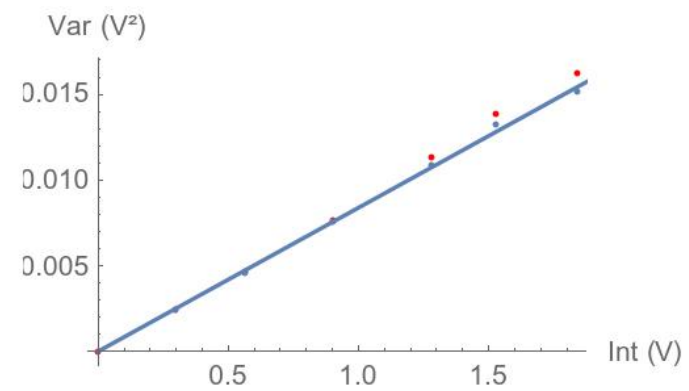

VictorSin

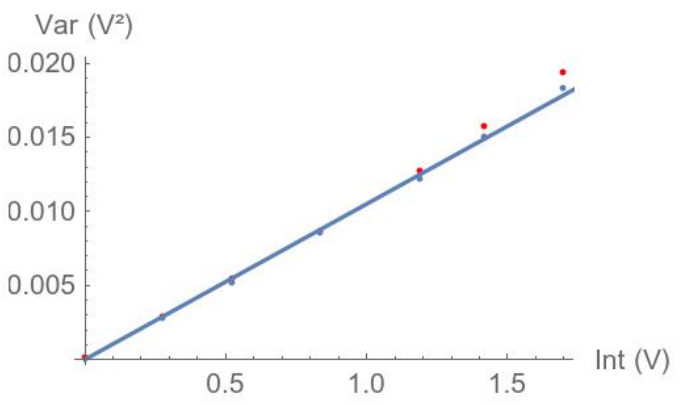

AliceSin

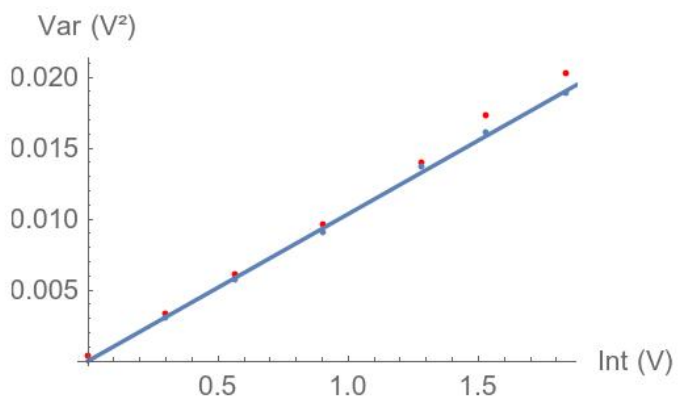

Figure 5.2: Example of shot noise measurement. The sum of the mean values (red points) and the their subtraction (blue points) are plotted against the measured variances. Horizontal axis shows intensity measurements, vertical axis shows variances measurements. All data is in units of volts as measured by the photodetectors.

\subsubsection{Teleportation analysis}

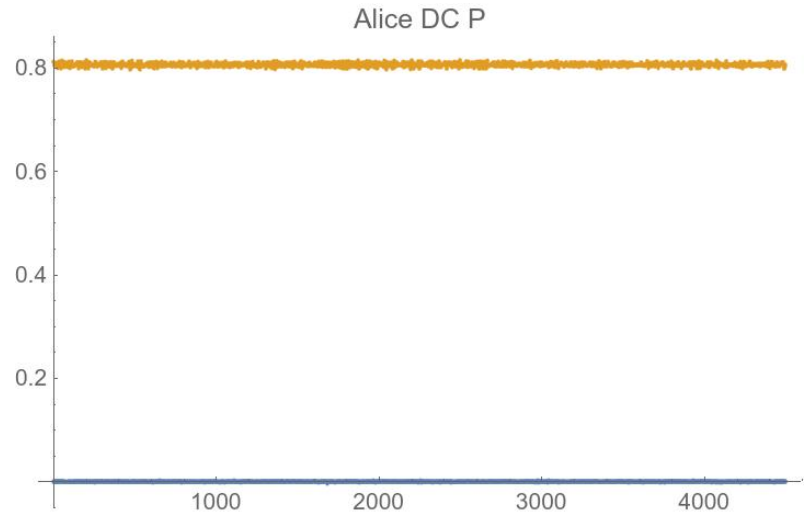

(a) Classical DC measurement for one detector.

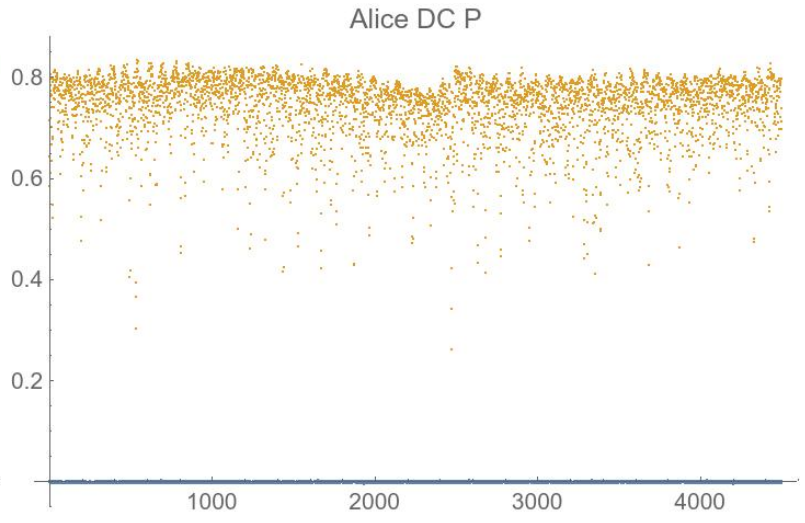

(b) Quantum DC measurement for the same detector.

Figure 5.3: Differences in noise from the laser's coherent beam and the OPO output. Blue is the dark measurement (measurement performed with no light) and yellow are the measured data with light. 

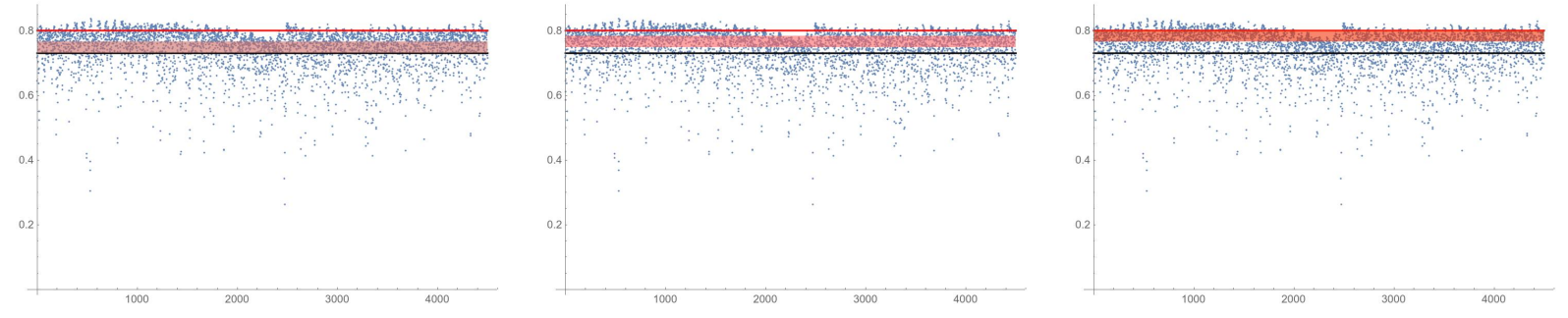

Figure 5.4: Data selection on a measurement of quantum teleportation. The data are the blue points, the red line shows which points are being considered on the analysis.

The noise present in the OPO output — as can be seen in fig (5.3) showing one $D C$ measurement of the experiment - is an indication of the difficulty in maintaining intensity stabilization. Since the measurements are never greater than $5 \%$ the threshold power, a shake in intensity may set the threshold limit at a power greater than the input pump, thus not generating twin beams. The picture compares the steady intensity measured during classical teleportation with the noisy measurement from an entangled beam. This troublesome effect is due to gray-tracking, explained on subsection (3.2.6), causing even noisier results on later measurements (appendix (D)). For the final measurements shown on the thesis, the treatment explained on the referred appendix was not necessary, only the data selection explained below was needed.

To solve this stability problem, data of quantum teleportation measurements were digitally divided on different parts, with each one being analysed separately as a different measurement. One of Alice's $D C$ measurement of the $\hat{P}$ quadrature was used to decide how the division of the data was performed. Each measurement was usually divided in three parts, where the difference from the point with the lowest intensity and the one with the highest was not greater than $40 \mathrm{mV}$ measured on the detector. Measurement points with big deviations from a mean value were excluded from the analysis. Fig (5.4) shows a possible way to filter the data for the quantum process of fig (5.3), leading to fig (5.5), exhibiting a scanning Victor cavity with the result of one of the selections in green.

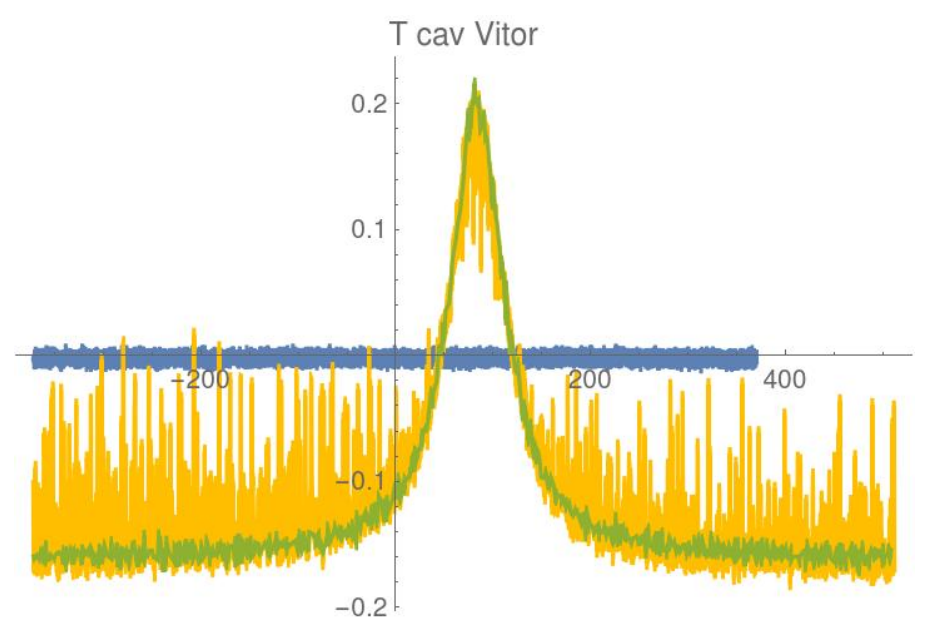

Figure 5.5: Victor's scanning cavity with the performed data selection. Blue points are dark, a measurement taken with the laser off so the zero point can be established; yellow points are all the measured data; and green is the leftover from the cut.

On the selected data, the calibrations defined earlier are applied for the final analysis to be performed. During teleportation measurement, each detector makes 450000 measurements on each run. The mean values and variances of the measured states are calculated by taking one point out of 100 
consequent ones. The graphs with the output states are built with 4500 points, less computationally demanding and not harmful for the data when the linewidth of the scanning Victor cavity is set big enough. All measurements must be subtracted from the dark measurement, performed when no light is sent to the detectors.
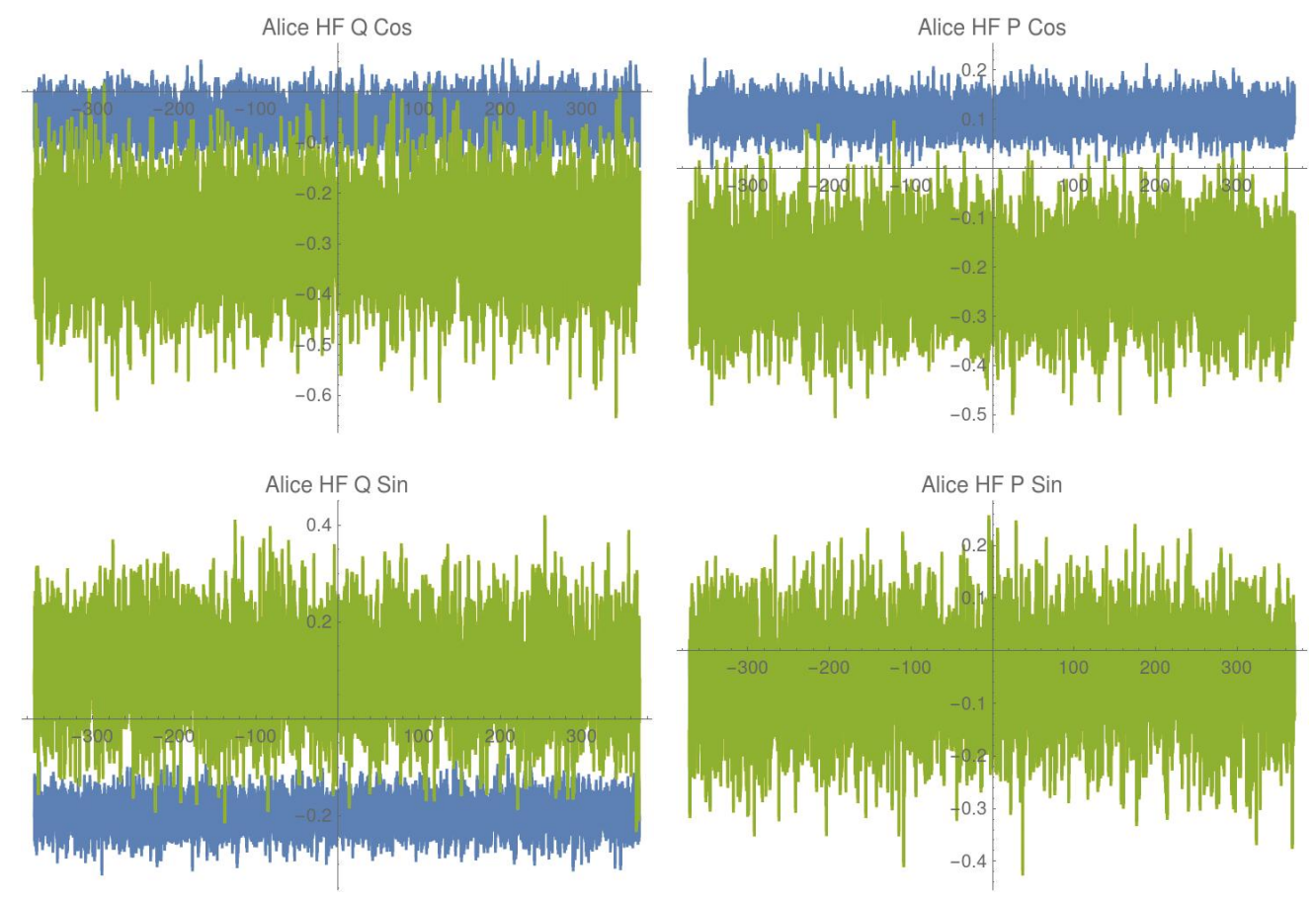

Figure 5.6: Input state of classical teleportation measured by Alice. Measured points are in green, blue points exhibit the electronic noise.

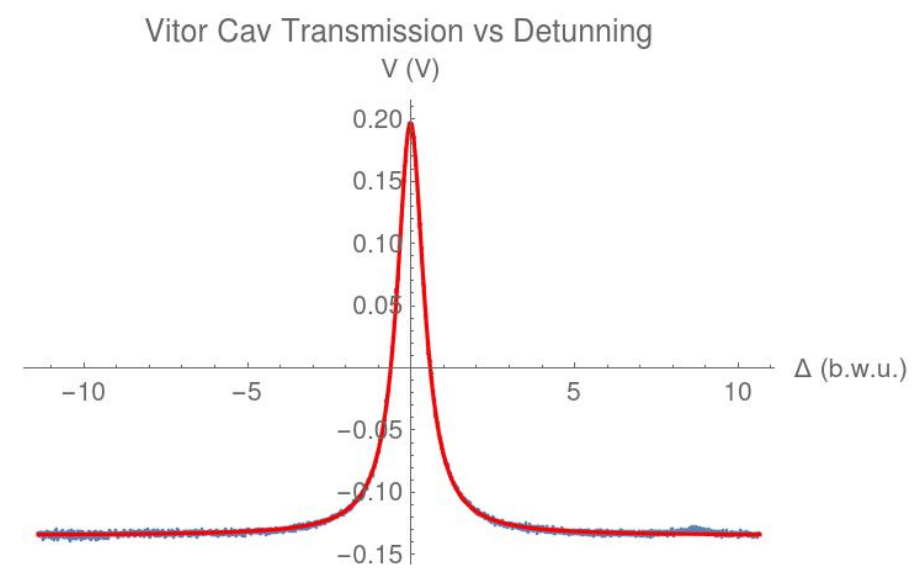

Figure 5.7: Transmission of Victor's scanning cavity as a function of detuning.

The input state is measured at Alice's station, resulting in $\hat{P}$ and $\hat{Q}$ quadratures demodulated in sine and cosine as shown in fig (5.6), where a classical teleportation measurement was chosen as example. The results of both mean values and variances are obtained from these graphs, by performing the correct operations. The labels on these figures have interchanged $\hat{P} \leftrightarrow \hat{Q}$, so the input state can be better compared with the output.

Victor's output measurements are obtained while his cavity scans (fig (5.7)). For the measurements of fig (5.6), his measurements are the ones presented on fig (5.8) for the mean values, and fig (5.9) for the variances. Since the input state has one quadrature measurement equal zero, $\hat{P}$ on the 
Mean value

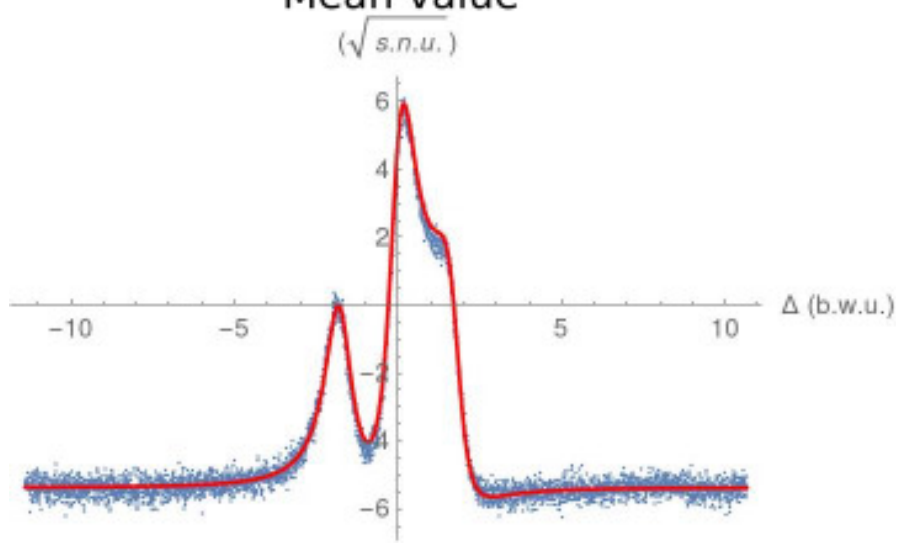

(a) Cosine demodulation

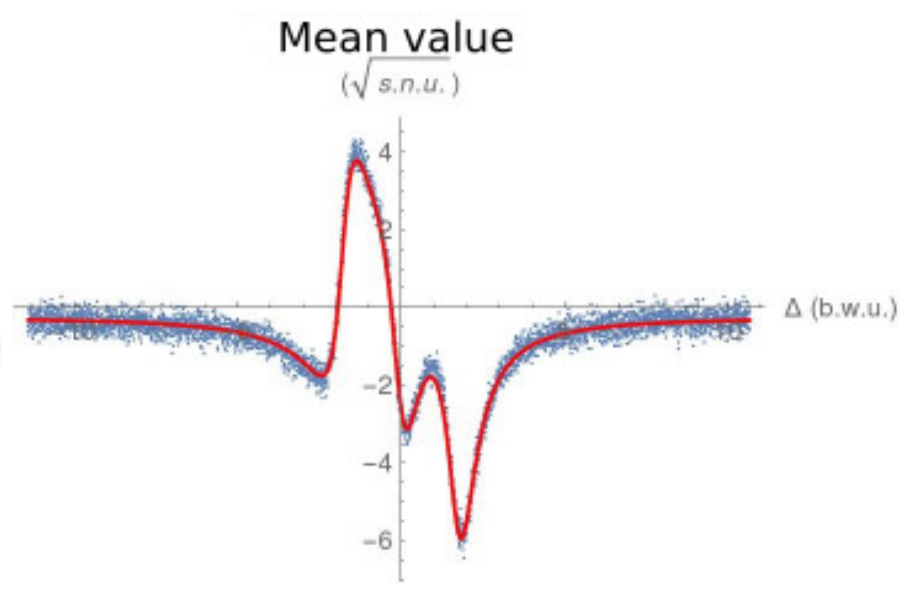

(b) Sine demodulation

Figure 5.8: Output state's mean value.

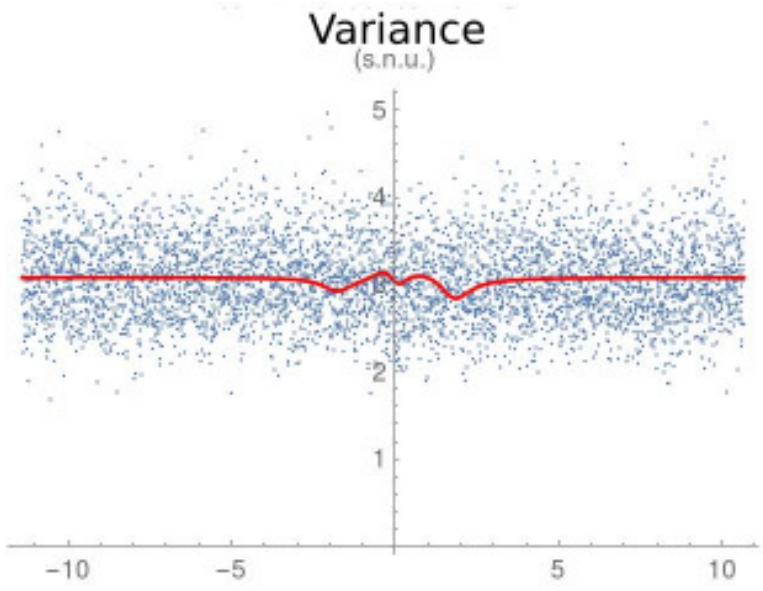

(a) Cosine demodulation

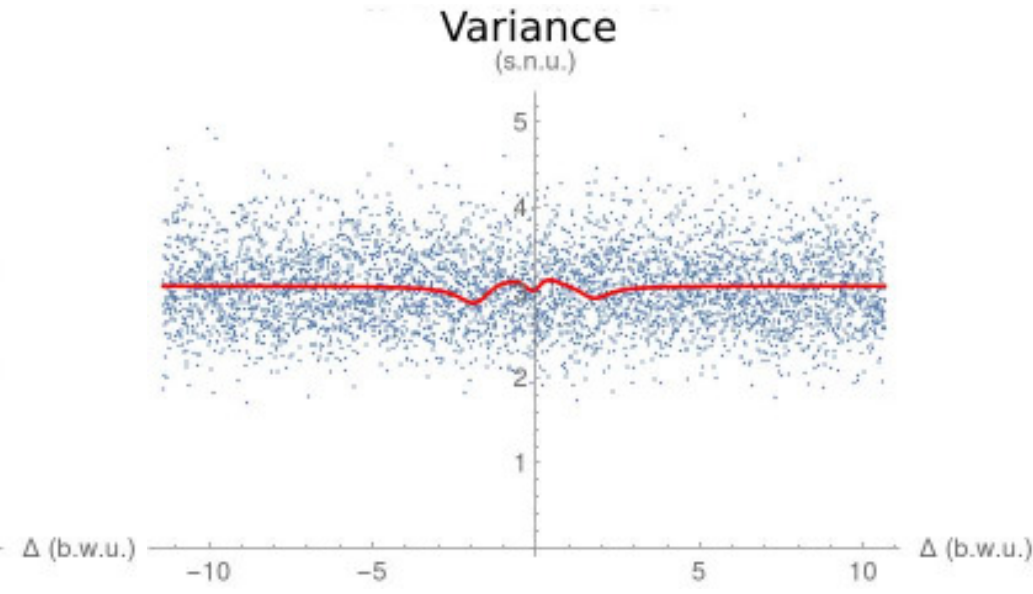

(b) Sine demodulation

Figure 5.9: Output state's variance.

sine demodulation, Victor's mean value measurement for the sine demodulation will also yield 0 when his cavity is away from $\Delta=0$ position, i.e., when the measured quadrature is $\hat{P}$. The different signs on Alice's $\hat{Q}$ measurements on each demodulation will result on peaks with different parities on Victor's output measurements. The output variances, as shown in fig (5.9), are roughly 3 for every combination of quadrature, as predicted on eqns (4.88) and (4.89).

\subsection{Classical teleportation}

After preliminary analysis of gain and shot noise adjustment is performed, teleportation results may be analysed. We will use the same classical teleportation measurement throughout the entire section as an example. Quantum teleportation results will be discussed on next section. On what follows, the labels $\hat{P}$ and $\hat{Q}$ for the input state are interchanged, so comparison with output results is clearer. Other interesting classical teleportation results are shown on appendix (国). 


\subsubsection{Mean values}

The mean values results are extracted from graphs as (5.6). Their behaviour, as intensity $\left|\alpha_{A}\right|^{2}$ increases, is dictated by eqns (2.79) and (2.80),

$$
\begin{gathered}
\left\langle\hat{P}_{i n}\right\rangle=-\sqrt{\left|\alpha_{A}\right|^{2}} K \sin \theta, \\
\left\langle\hat{Q}_{i n}\right\rangle=\sqrt{\left|\alpha_{A}\right|^{2}} K \cos \theta,
\end{gathered}
$$

where $K=2 M$ is related to the voltage applied on the function generator. The position $\theta$ of the MachZehnder locking is subject to fluctuations during a collection of measurements, leading to fluctuations on the quadratures' mean values as shown on fig (5.10), where input quadratures are shown for the uncorrected input state. For the data on the graphs, the modulation resulted in a stable value for the $\left\langle\hat{P}_{i n}\right\rangle$ quadrature. However, the tiny contribution to the $\left\langle\hat{Q}_{i n}\right\rangle$ quadrature made it more sensitive to fluctuations on the locking.

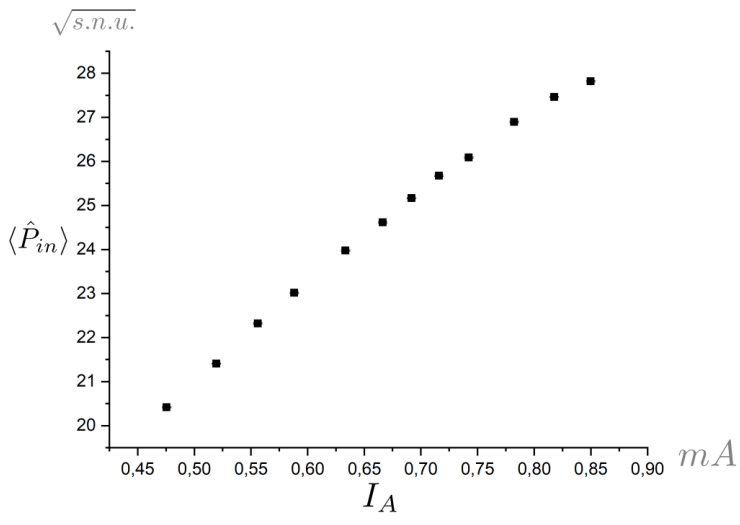

(a) $\left\langle\hat{P}_{i n}\right\rangle$ quadrature.

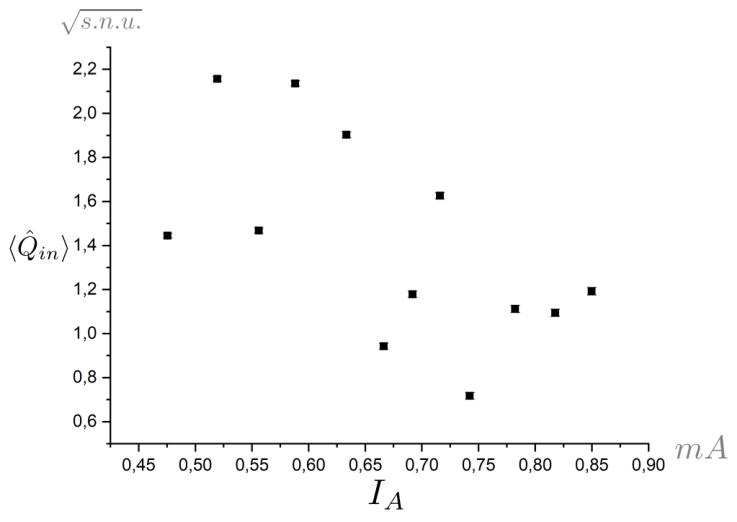

(b) $\left\langle\hat{Q}_{i n}\right\rangle$ quadrature.

Figure 5.10: Amplitude measurement of the input state for each quadrature, on square root of shot noise units, as a function of the $D C$ photocurrent measured on Alice's station in miliamperes units, proportional to the intensity $\left|\alpha_{A}\right|^{2}$ (eqn (2.49)).

For this reason, instead of analysing each quadrature individually, in a first approach we may consider only the amplitude of the state for analysis,

$$
\xi_{i n}=\sqrt{\left\langle\hat{P}_{i n}\right\rangle^{2}+\left\langle\hat{Q}_{i n}\right\rangle^{2}}=K \sqrt{\left|\alpha_{A}\right|^{2}}
$$

getting rid of the dependence from $\theta$. The data of fig (5.10) is shown on fig (5.11) for $\xi_{\text {in }}$, where eqn (5.4) was fitted with $K$ as the only free parameter.

The output state quadratures have a resemblant behaviour as the input quadratures as a function of the measured photocurrent, seen on fig (5.12). Its values are extracted from graphs as fig (5.8). Similarly as the treatment done to the input state, we will analyse the amplitude $\xi_{\text {out }}=\Gamma \xi_{\text {in }}$ (section (4.7)). Since $\Gamma$ must be linear to the intensity and $\left|\alpha_{A}\right|^{2} \approx\left|\alpha_{B}\right|^{2}$, it is possible to write

$$
\xi_{\text {out }}=K^{\prime}\left(\sqrt{|\alpha|^{2}}\right)^{3} \text {, }
$$

where $K^{\prime}$ is related to $K$ from eqn (5.4) as well as the proportional factor between gain $\Gamma$ and intensity $\left|\alpha_{B}\right|^{2}$. Plots are shown on fig (5.13), where $K^{\prime}$ is the only free parameter. 


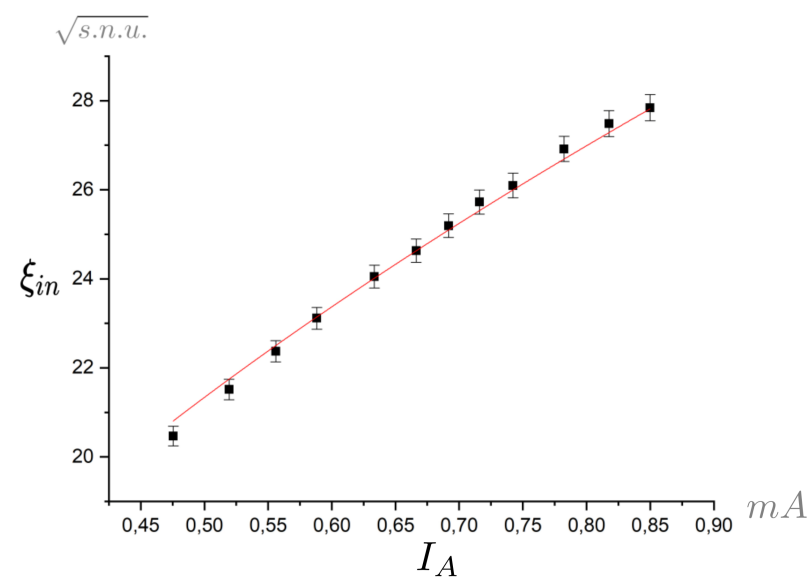

Figure 5.11: $\xi_{\text {in }}$, in square root of shot noise units, as a function of the photocurrent measured by Alice in miliamperes.

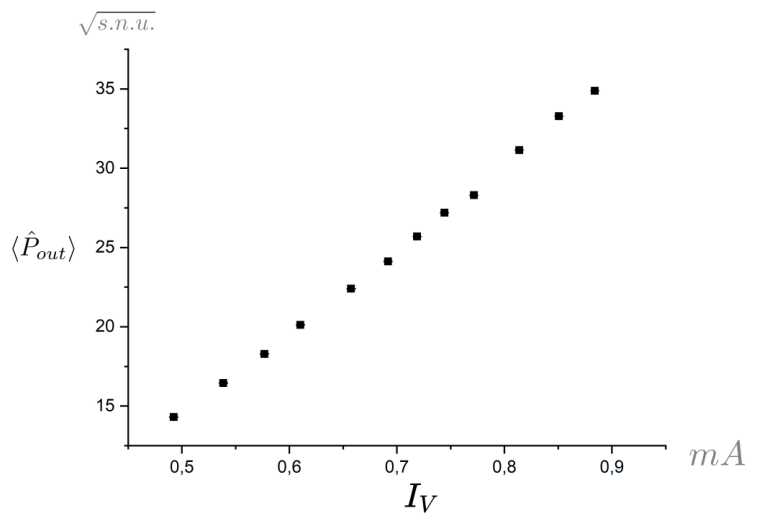

(a) $\left\langle\hat{P}_{\text {out }}\right\rangle$ quadrature.

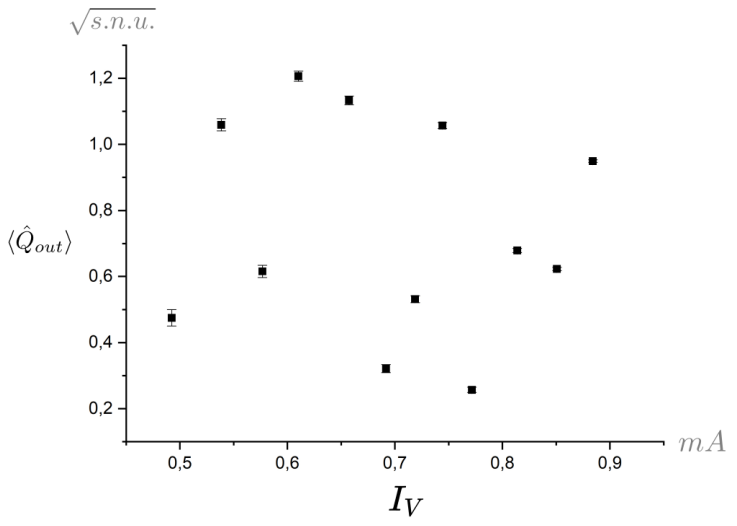

(b) $\left\langle\hat{Q}_{\text {out }}\right\rangle$ quadrature.

Figure 5.12: Quadratures of output states for cosine demodulation, on square root of shot noise units, as a function of the measured photocurrent on Victor's station in miliamperes.

The values of $\Gamma$ may be calculated from the photocurrent measurements by comparing the input and output states' amplitudes, as can be seen on fig (5.14), which plots both results of fig (5.13) and fig (5.11) on the same graph. When the gains are perfectly adjusted (which we associate to the gain value $\Gamma=1$ ) we must have $\xi_{\text {out }}=\xi_{\text {in }}$, the same amplitude must be measured on input and output. We associate the photocurrent value for which this happens to $\Gamma=1$ and normalize all other photocurrent measurements accordingly.

The preliminary analysis of gain and shot noise adjustment dictates how big a difference between measurements on each electronic demodulation is acceptable. On this experiment, it is not expected differences bigger than $5 \%$ between sine and cosine demodulated signals. 


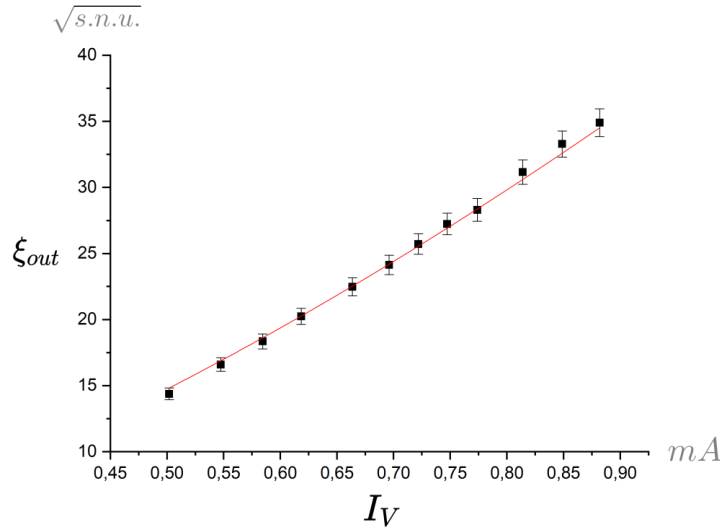

(a) Cosine demodulation.

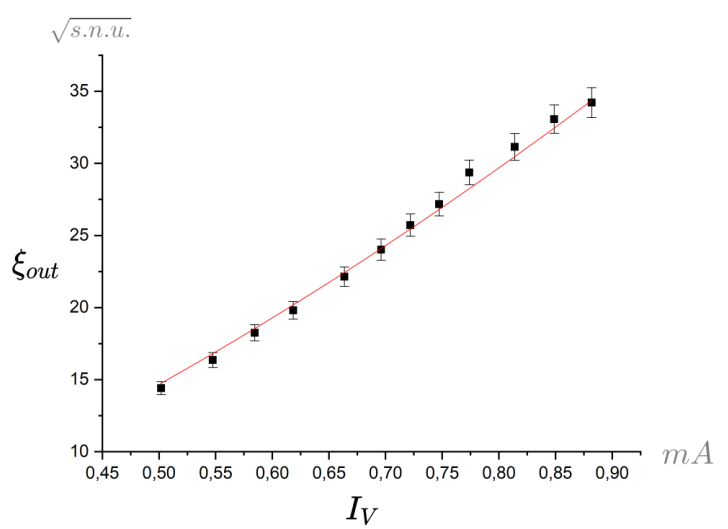

(b) Sine demodulation.

Figure 5.13: Measurement of $\xi_{\text {out }}$ on square root of shot noise units for each electronic quadrature as a function of the measured photocurrent in miliamperes.

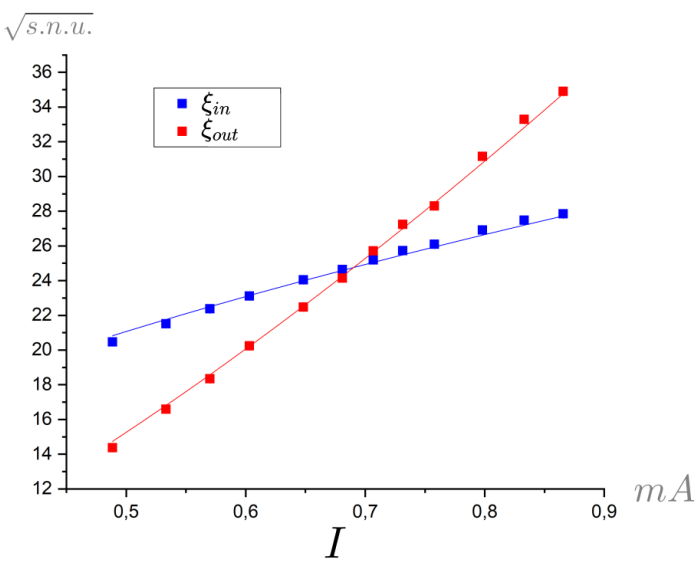

(a) Cosine demodulation.

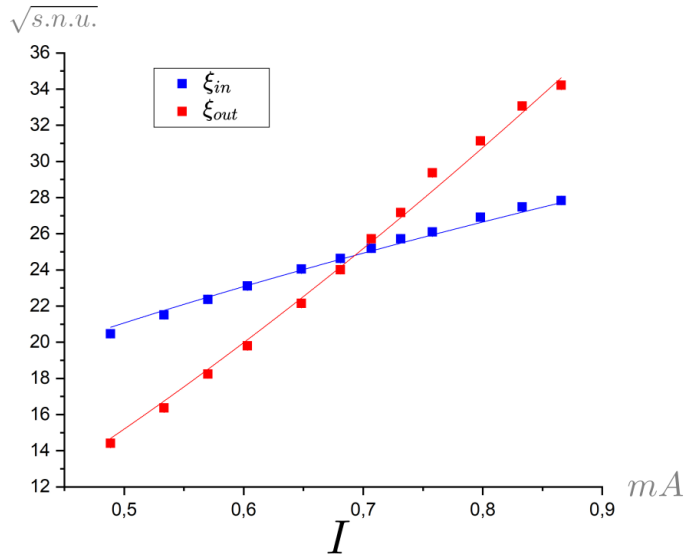

(b) Sine demodulation.

Figure 5.14: $\xi_{\text {in }}$ and $x i_{\text {out }}$ plotted on the same graph, on square root of shot noise units, for each demodulation as a function of the measured photocurrent in miliamperes. 


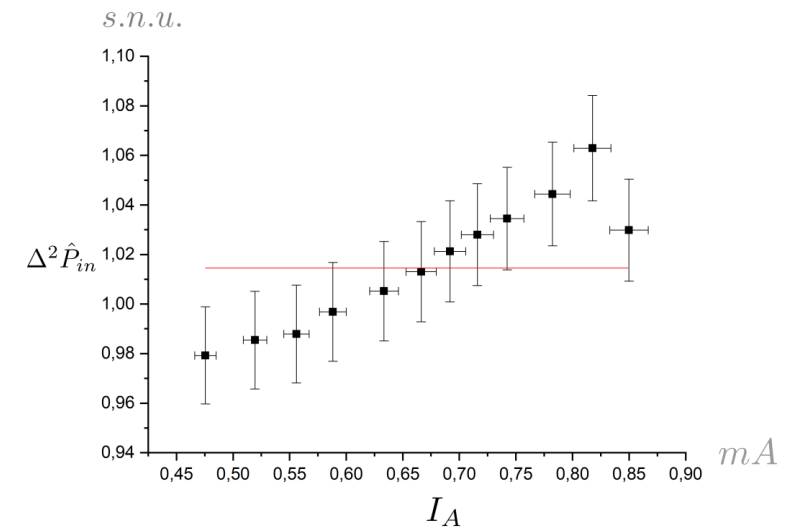

(a)

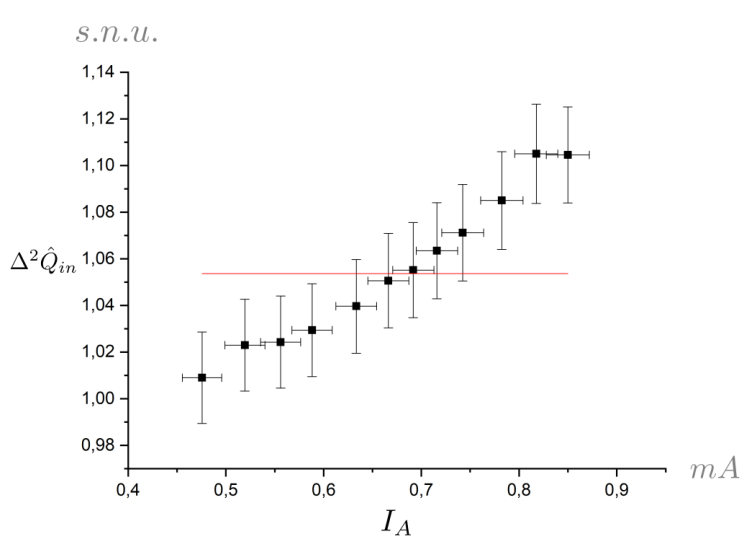

(b)

Figure 5.15: Alice's variance measurements in shot noise units, against the measured photocurrent in miliamperes.

Alice's measurements of the variance of the input state are expected to result in a constant value of 1 in shot noise units. Fig (5.15) shows the experimental data for a day of measurements. On average over a set of different measurements, the fitted value for a constant line on the plot was 1.01(1) for $\Delta^{2} \hat{P}_{i n}$ and $1.05(1)$ for $\Delta^{2} \hat{Q}_{i n}$. It is possible to see a tendency on the variances to increase with intensity. If the angular coefficient of a straight line is set free for fitting, the obtained result is, on average over a set of different measurements, $0.13(2)$ for $\Delta^{2} \hat{P}_{i n}$, and $0.20(2)$ on $\Delta^{2} \hat{Q}_{i n}$.

The input state's measurements are sent to Bob for the construction of the output state. For this reason, output Victor's variances will be related to gain $\Gamma$. Under classical teleportation, with no squeezing, the behaviour of Victor's measurements is predicted by eqn (4.68) for the uncorrected input state. This equation is obtained from the discussion on subsection (4.4.2). Victor's variances $\sigma_{V}$ are related to the $\Gamma$ by

$$
\sigma_{V}=1+2 \Gamma^{2}+E^{2} \Gamma
$$

where $E^{2}$ is the electronic noise sent from Alice to Bob along with her measured results (eqn (4.65)). Even though this undesired parameter may be subtracted from Alice's measurements during analysis of the measurements, it is present on the photodetectors and amplified by $\Gamma$ when sent to Bob. Fig (5.16) plots this equation along with the experimental points with no free parameters, the graph is used as a comparison between expectation and measurement. The great agreement between prediction and experiment seen on the graph indicates a good gain adjustment. The values of gain $\Gamma$ were derived from the graphs on fig (5.11), setting $\Gamma=1$ at an intensity $|\alpha|^{2}=0.69 \mathrm{~V}$. 


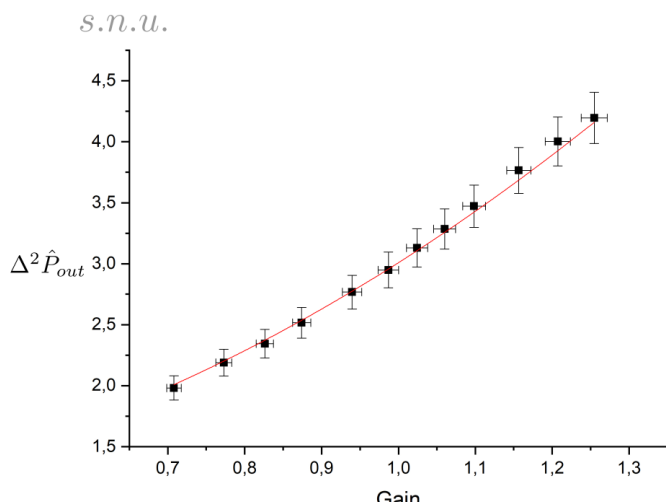

(a) Cosine demodulation.

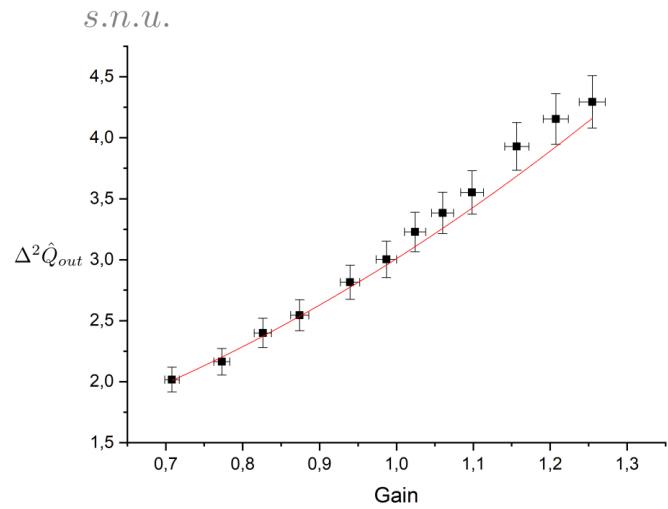

(c) Cosine demodulation.

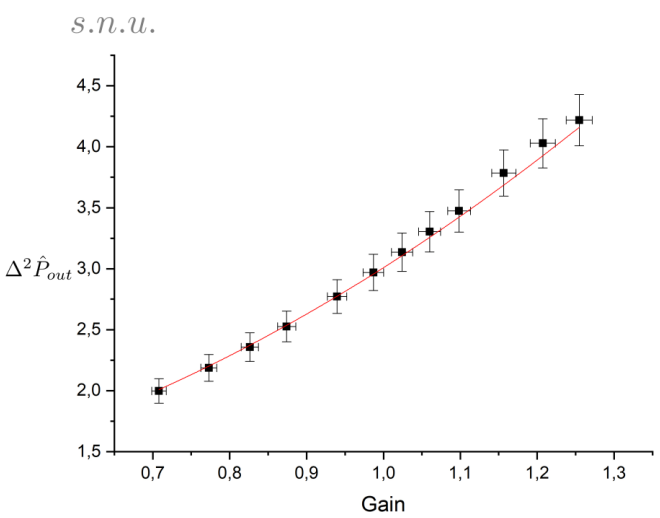

(b) Sine demodulation.

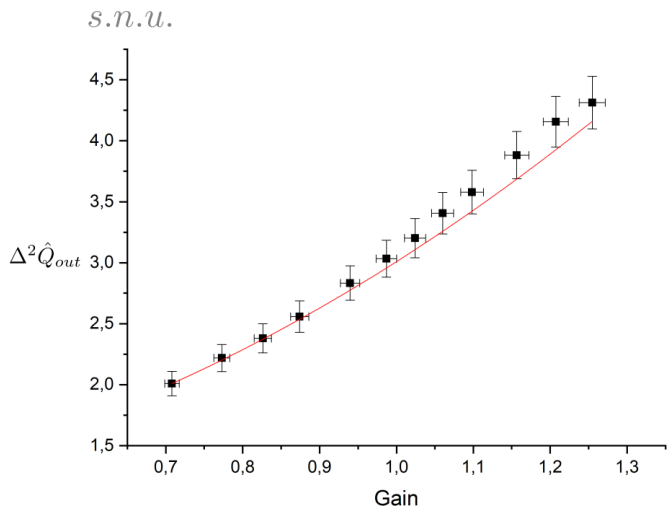

(d) Sine demodulation.

Figure 5.16: Victor's variance measurements, in shot noise units, against gain $\Gamma$, as defined by the mean values' plots.

\subsubsection{Teleportation for the uncorrected input state}

Vacuum teleportation, shown on fig (5.17), is a quantity related only to the variances' measurements. It is calculated by

$$
\mathcal{F}_{\text {vac }}=\frac{2}{\sqrt{\left(1+\Delta^{2} \hat{P}_{\text {out }}\right)\left(1+\Delta^{2} \hat{Q}_{\text {out }}\right)}},
$$

where, on the classical case, both quadratures variances behave as eqn (5.6). As before, the curve is plotted along the experimental points with no free parameters, so deviations from prediction can be visualized. For the data in question, there is a big agreement between measured points and the theoretical curve. This plot again indicates a great quality of the adjustment of $\Gamma$. 


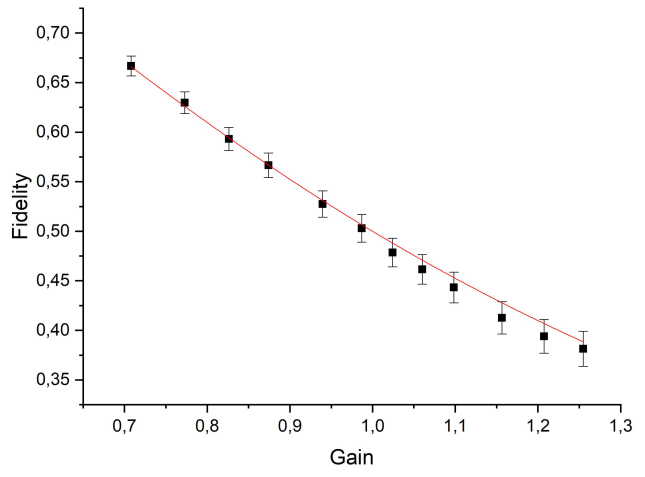

(a) Cosine demodulation.

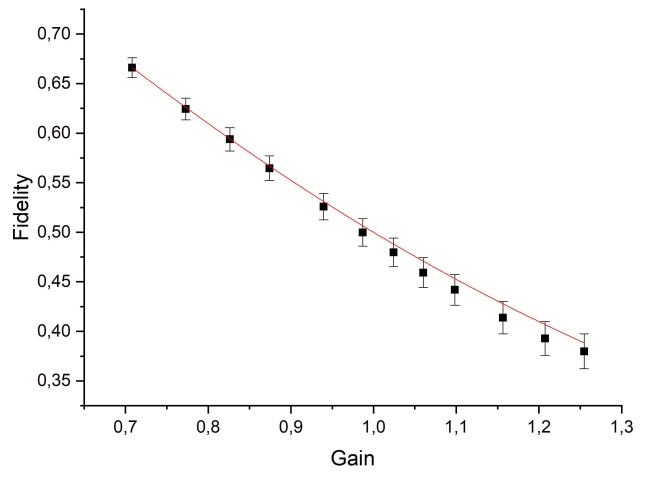

(b) Sine demodulation.

Figure 5.17: Vacuum teleportation.

When the mean values are taken into account, we return to the fidelity equation (4.78), leading to the graphs of fig (5.18), for the uncorrected input state. The curve is the same used on fig (4.11), using the measured mean values for this state. It is plotted with no free parameters along the experimental data for comparison. Imperfections to the gain adjustment caused the observed differences between the predicted results and the obtained ones.

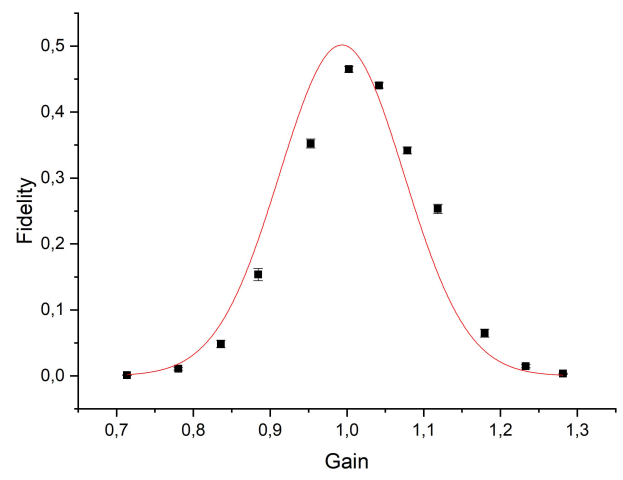

(a) Cosine demodulation.

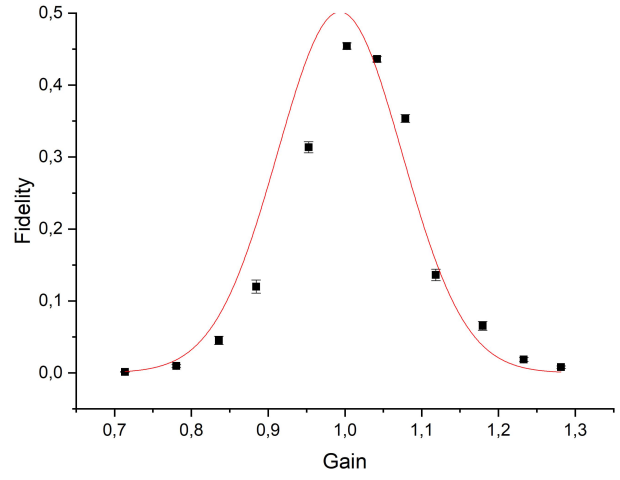

(b) Sine demodulation.

Figure 5.18: Coherent state teleportation.

The measured data closer to $\Gamma=1$ is a point measured at $\Gamma=1.002(5)$, which results in

$$
\mathcal{F}_{\cos }(1.002)=0.465(4), \quad \mathcal{F}_{\sin }(1.002)=0.454(4) .
$$

\subsubsection{Teleportation for the corrected Input state}

The uncorrected input state shown here has the following properties around $\Gamma=1$, measured in square roots of shot noise unit,

$$
\left\langle\hat{P}_{i n}\right\rangle \approx 24.6, \quad\left\langle\hat{Q}_{i n}\right\rangle \approx 0.9, \quad \xi_{\text {in }} \approx 24.6
$$

If we are to correct these numbers to retrieve the state generated by Victor on the input station, Alice's detection efficiency comes as a loss factor (subsection (4.4.2)). The input amplitude is transformed as

$$
\xi_{\text {in }} \approx 24.6 \rightarrow \xi_{\text {in }} \approx 33.9 .
$$


Variances are expected to follow equation (4.69), predicting a measurement of 3.759 s.n.u. for Victor's variances at $\Gamma=1$. This imposes a limit of 0.420 for classical teleportation fidelity at unity gain. As explained on subsection (4.4.2), $\Gamma$ is rescaled accordingly with Alice's detection efficiency, resulting in the graphs shown on fig (5.19), where the curves are given by the same equations as before and are also plotted with no free parameters.

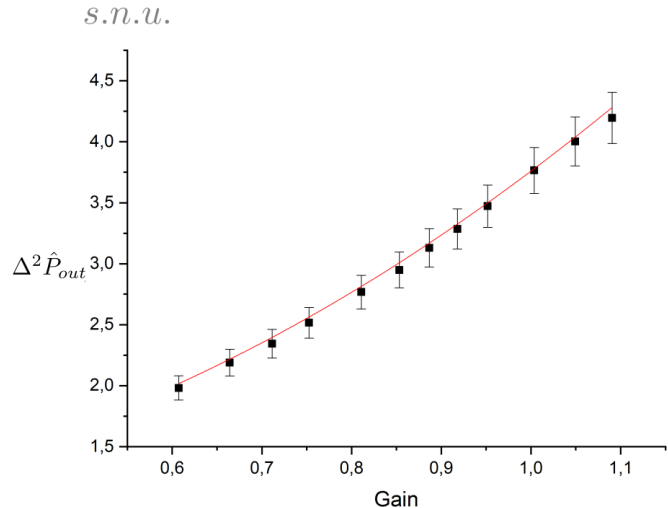

(a) Measured output variances

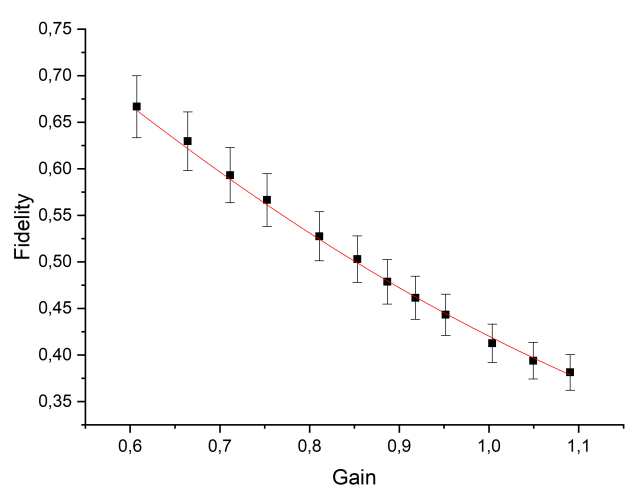

(b) Vacuum teleportation.

Figure 5.19: Cosine demodulation of discussed data considering the input state as the one generated by Victor instead of the one measured by Alice. Plot done with no free parameters.

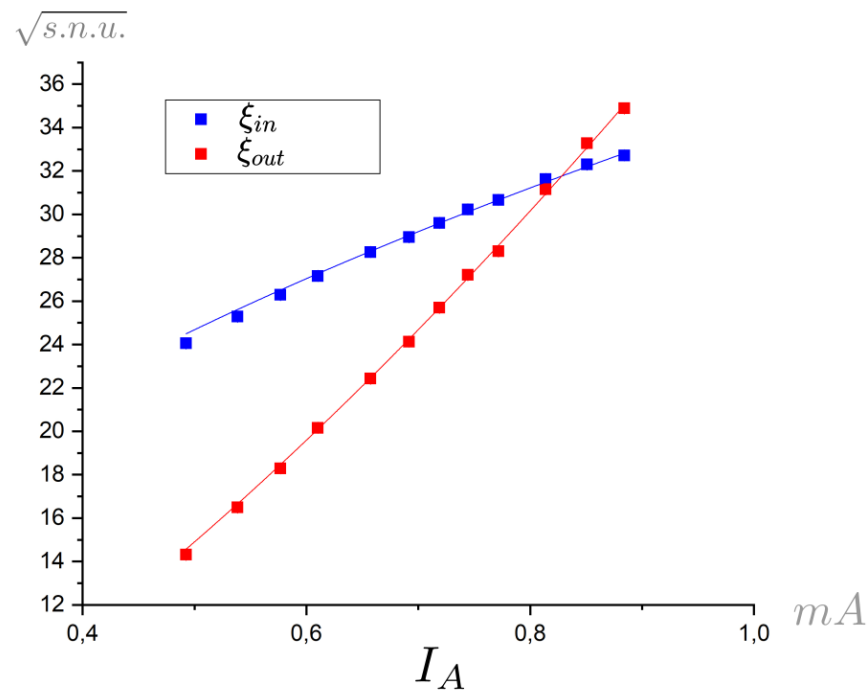

Figure 5.20: Input and output mean values plotted against the measured photocurrent in miliamperes.

The mean values behaviours are shown in fig (5.20), with the fitting performed as explained on subsection (5.2.1). This rescaling led to values of $\Gamma$ around $15 \%$ greater than before. Fidelity results are

$$
\mathcal{F}_{\cos }(1.003)=0.388(3), \quad \mathcal{F}_{\text {sin }}(1.003)=0.395(3)
$$

\subsubsection{Two-mode analysis}

So far, teleportation analysis was done considering one mode per beam with two quadratures, $\hat{P}$ and $\hat{Q}$. For a more general analysis, we need to consider the quadrature information of each sideband 
of each beam. This leads to a two-mode analysis, with four quadratures $-\hat{p}_{s}, \hat{q}_{a}, \hat{q}_{s}$ and $\hat{p}_{a}$. After a modulation, these quadratures are expected to be populated as shown on subsection (2.7.2).

$$
\begin{aligned}
\left\langle\hat{p}_{s}\right\rangle=-\sqrt{\Gamma} K \cos \phi \sin \theta, & \left\langle\hat{q}_{a}\right\rangle=\sqrt{\Gamma} K \sin \phi \sin \theta, \\
\left\langle\hat{q}_{s}\right\rangle=\sqrt{\Gamma} K \cos \phi \cos \theta, & \left\langle\hat{p}_{a}\right\rangle=-\sqrt{\Gamma} K \sin \phi \cos \theta,
\end{aligned}
$$

where the parameter $\phi$ is the modulation phase.

Under one-mode analysis on previous subsections, we defined $\xi=\sqrt{\langle\hat{P}\rangle^{2}+\langle\hat{Q}\rangle^{2}}$ for the study of the mean values. Following the same logic, we could define

$$
\xi_{s}=\sqrt{\left\langle\hat{p}_{s}\right\rangle^{2}+\left\langle\hat{q}_{s}\right\rangle^{2}}=K \sqrt{\Gamma} \cos \phi, \quad \xi_{a}=\sqrt{\left\langle\hat{p}_{a}\right\rangle^{2}+\left\langle\hat{q}_{a}\right\rangle^{2}}=K \sqrt{\Gamma} \sin \phi,
$$

the amplitudes of the symmetric and antisymmetric combinations of quadratures. This definition gets rid of the $\theta$ dependence, a number that may fluctuate in a data collection, as already seen. The amplitude $\xi$ of one-mode analysis is related to the above by $\xi=\sqrt{\xi_{s}^{2}+\xi_{a}^{2}}$.

If the definitions were made to get rid of $\phi$ instead of $\theta$, we would simply return to the one-mode quadratures' definitions

$$
\langle\hat{P}\rangle=\sqrt{\left\langle\hat{p}_{s}\right\rangle^{2}+\left\langle\hat{q}_{a}\right\rangle^{2}}=K \sqrt{\Gamma} \sin \theta, \quad\langle\hat{Q}\rangle=\sqrt{\left\langle\hat{q}_{s}\right\rangle^{2}+\left\langle\hat{p}_{a}\right\rangle^{2}}=K \sqrt{\Gamma} \cos \theta,
$$

with a behaviour shown on fig (5.10).

Graphs for $\xi_{s}$ and $\xi_{a}$ for the example state of this section are shown of fig (5.21) where the values for gain $\Gamma$ are the same defined on one-mode analysis considering the input state as the one generated by Victor. Their behaviours are not exactly proportional to $\sqrt{\Gamma}$ for the input quadratures and $\sqrt{\Gamma^{3}}$ for the output. A possible explanation comes from deviations on phase $\phi$. However, it is still possible to see how the amplitude of the output state is closer to the input when the gain is closer to unity.

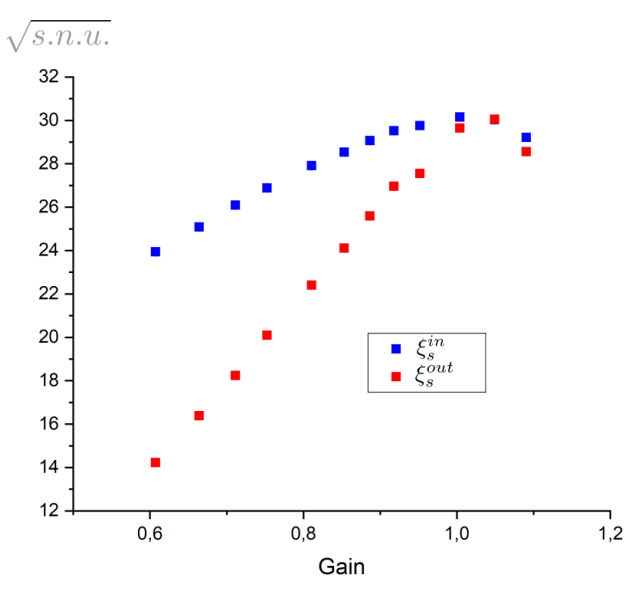

(a) Symmetric combination of quadratures. $\xi_{s} \approx 30$ at $\Gamma=1$.

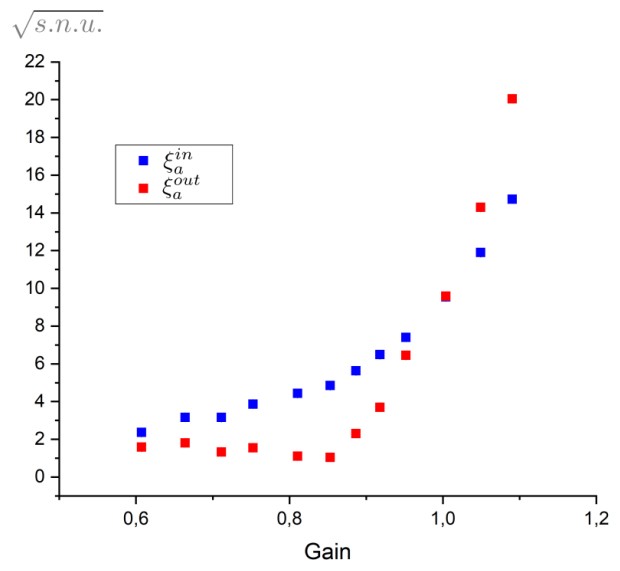

(b) Antisymmetric combination of quadratures. $\xi_{a} \approx 9.5$ for $\Gamma=1$

Figure 5.21: Amplitudes for the symmetric and antisymmetric combination of quadratures considering the input state as the one generated by Victor.

For unitary gain, the measured input quadratures are

$$
\begin{array}{rlrl}
\left\langle\hat{p}_{s}\right\rangle \approx 30, & \left\langle\hat{q}_{a}\right\rangle & \approx 9.5, \\
\left\langle\hat{q}_{s}\right\rangle \approx-1.0, & \left\langle\hat{p}_{a}\right\rangle \approx-0.8 .
\end{array}
$$


The obtained fidelities for the symmetric (indice $s$ ) and antisymmetric (indice $a$ ) combination of quadratures are

$$
\begin{array}{ll}
\mathcal{F}_{\cos }^{s}(1.003)=0.393(3), & \mathcal{F}_{\sin }^{s}(1.003)=0.393(3), \\
\mathcal{F}_{\text {cos }}^{a}(1.003)=0.405(3), & \mathcal{F}_{\sin }^{a}(1.003)=0.376(3) .
\end{array}
$$

\subsection{Quantum teleportation}

Before performing quantum teleportation, a classical measurement is performed to experimentally define the value of $\Gamma$ and check if the system is working properly. The mean values of the input and output states of this test measumeremnt are shown shown on fig (5.22), considering the uncorrected input state. This graph defines the values of $\Gamma$ for all subsequent measurements of the day. The fitting is performed as explained on subsection (5.2.1).

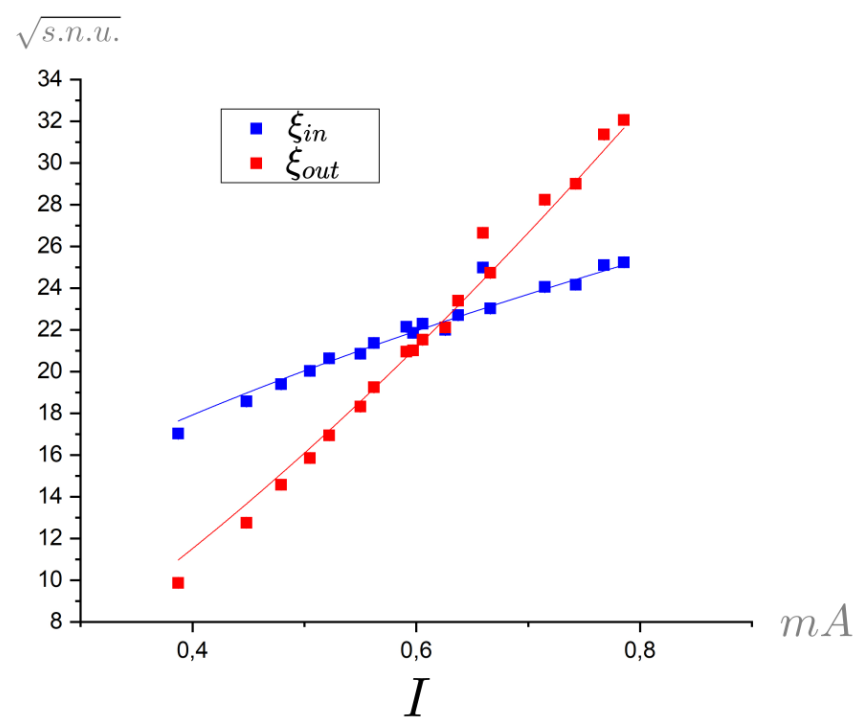

Figure 5.22: Mean values of classical teleportation data considering the uncorrected input state. $\xi \approx 22$ at $\Gamma=1$.

The behaviour of the output variances are shown on fig (5.23) for the cosine demodulation, where the plotted curve is eqn (5.6) with no free parameters. The gain adjustment have different qualities for each quadrature, with the biggest deviation from ideal due to $\Delta^{2} \hat{P}$ measurements.

The measured points closer to unitary gain are $\Gamma=0.994(9)$ and $\Gamma=1.012(9)$, leading to fidelity results on table 5.1 for the comparison with the uncorrected input state. Even with non ideal gain adjustment, measurements of fidelity are close to the expected classical limit.

\begin{tabular}{c|c|c|c} 
& One-mode & Symmetric combination & Antisymmetric combination \\
$\mathcal{F}(0.994)$ & $0.434(4)$ & $0.456(7)$ & $0.40(1)$ \\
$\mathcal{F}(1.012)$ & $0.42(3)$ & $0.438(7)$ & $0.376(4)$
\end{tabular}

Table 5.1: Fidelities averaged over sine and cosine demodulations.

For the corrected input state, table (5.1) is updated to table (5.2), where the point $\Gamma=1.003(9)$ was measured. 


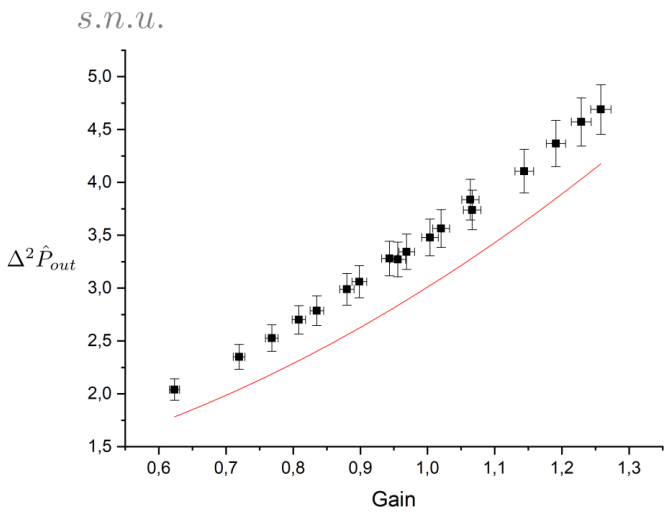

(a) Cosine demodulation of $\Delta^{2} \hat{P}_{\text {out }}$ measurement.

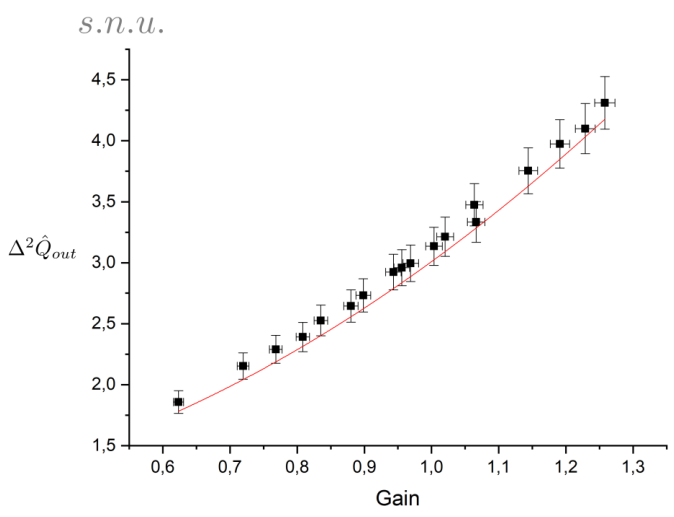

(b) Cosine demodulation of $\Delta^{2} \hat{Q}_{\text {out }}$ measurement.

Figure 5.23: Output variances of classical teleportation for the uncorrected input state.

$$
\begin{array}{c|c|c|c} 
& \text { One-mode } & \text { Symmetric combination } & \text { Antisymmetric combination } \\
\mathcal{F}(1.003) & 0.36(3) & 0.380(6) & 0.34(3)
\end{array}
$$

Table 5.2: Fidelities averaged over sine and cosine demodulations.

\subsubsection{Comparison between classical an quantum teleportation}

The experimental equipment is sensitive to the adjustment conditions. To avoid problemas arising from fluctuations on the settings, both classical and quantum teleportation were performed on the same day under the same conditions. The input state of both measurements must be similar, as shown on graph (a) of fig (5.24). However, this is not true for the output state, as shown on graph (b). This happens because the beams generated by the OPO have an unbalance of $15 \%$ in energy, as opposed to the classical teleportation performed with beams with the same energy. Both these graphs were fitted by the equations for $\xi_{\text {in }}$ and $\xi_{\text {out }}$ exposed on subsection (5.2.1).

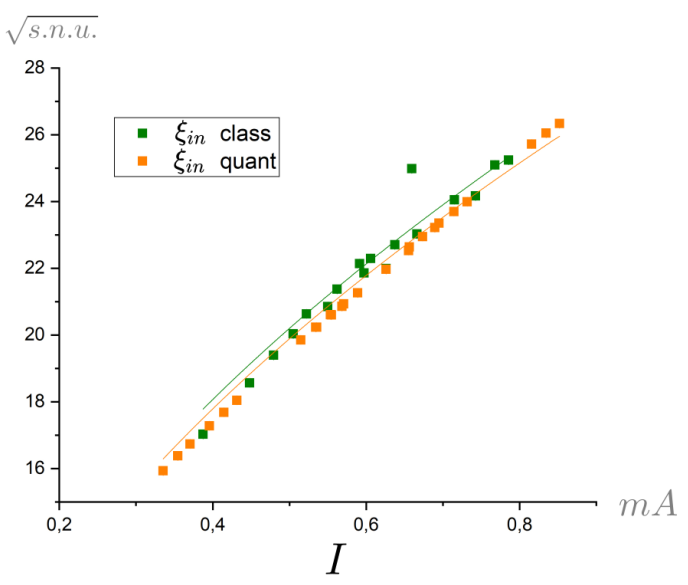

(a) Input state.

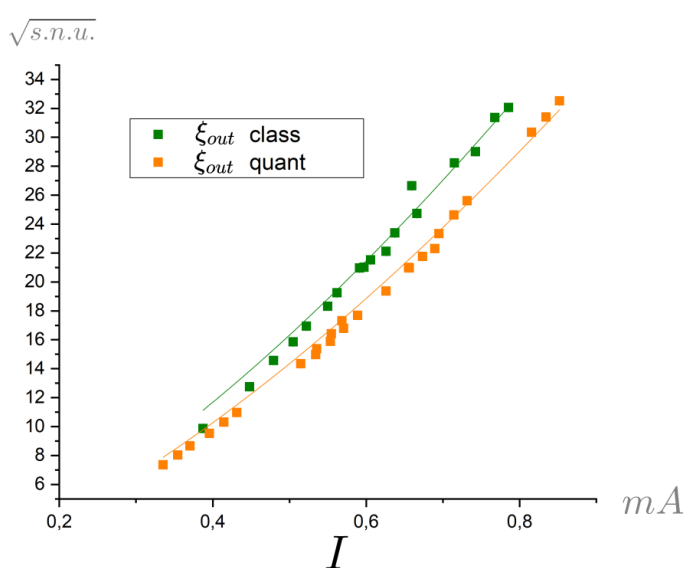

(b) Output state.

Figure 5.24: Input and output states compared between classical and quantum teleportation setups.

The value of $\Gamma$ derived from the classical teleportation measurement may be compared with the value of $\Gamma$ we would obtain if we derived it from the quantum teleportation's measurement of fig (5.25). The latter definition results in values of gain $4 \%$ bigger than the definition by classical teleportation. 


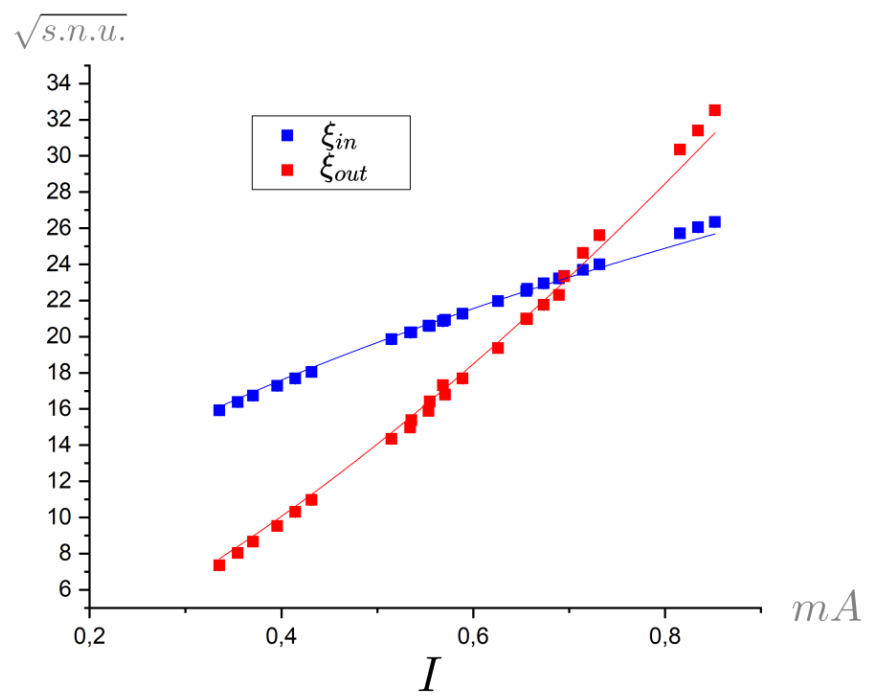

Figure 5.25: Input and output states of quantum teleportation for the uncorrected input state. Cosine demodulation.

\subsubsection{Quantum teleportation}

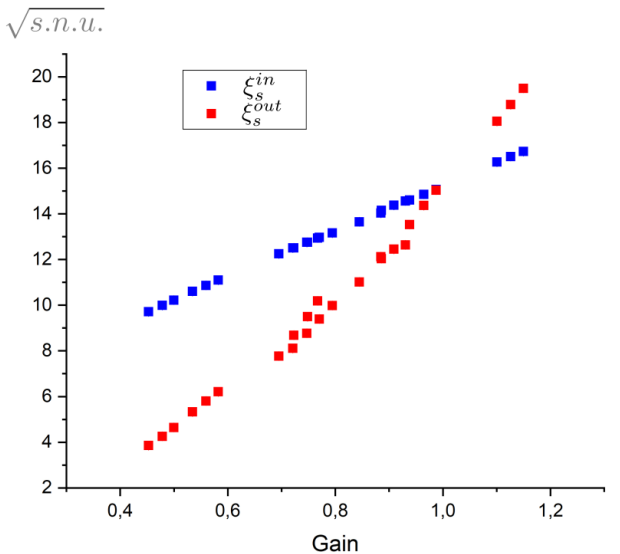

(a) Cosine demodulation of symmetric combination of quadratures. $\xi_{s} \approx 15$ at $\Gamma=1$.

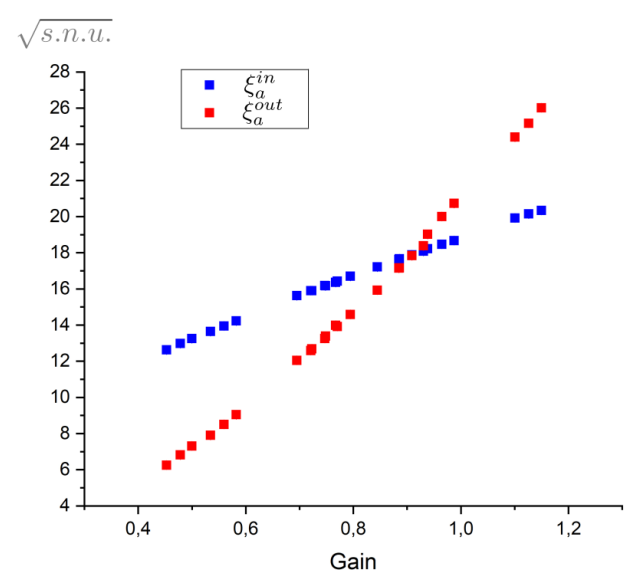

(b) Cosine demodulation of antisymmetric combination of quadratures. $\xi_{a} \approx 19$ at $\Gamma=1$.

Figure 5.26: Input and output states of quantum teleportation for the uncorrected input state.

Graphs for the amplitudes $\xi_{s}$ and $\xi_{a}$ for quantum teleportation are shown on fig (5.26) for the uncorrected input state. The input quadratures of the setup are

$$
\begin{aligned}
& \langle\hat{P}\rangle \approx 24, \\
& \langle\hat{Q}\rangle \approx 3, \\
& \left\langle\hat{p}_{s}\right\rangle \approx 15 \text {, } \\
& \left\langle\hat{q}_{a}\right\rangle \approx-18 \text {, } \\
& \left\langle\hat{q}_{s}\right\rangle \approx-2, \\
& \left\langle\hat{p}_{a}\right\rangle \approx 3 .
\end{aligned}
$$

The measurement of the input variances are shown on fig (5.27) along with a constant line plot. The value greater than 1 indicates that Victor's beam is mixed with a thermal state. The output variances are plotted on fig (5.28) along with the theoretical curve predicted on eqn (4.98) with no free parameters. By using this equation, all losses terms discussed on subsection (4.7.2) are taken into account. The values 


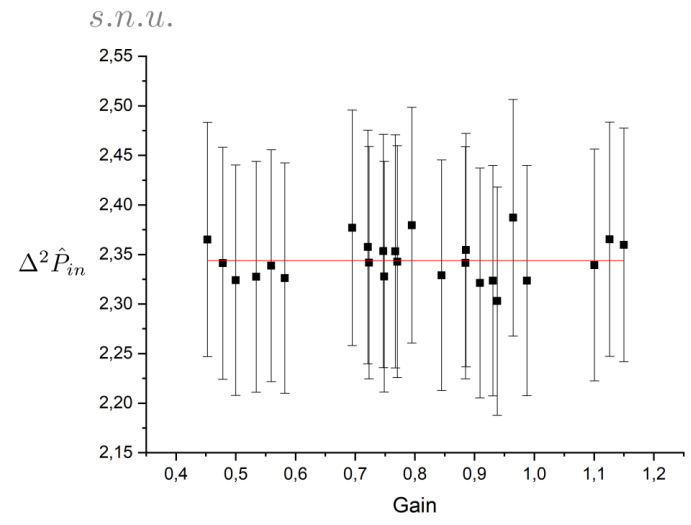

(a) Input variances of the $\hat{P}$ quadrature.

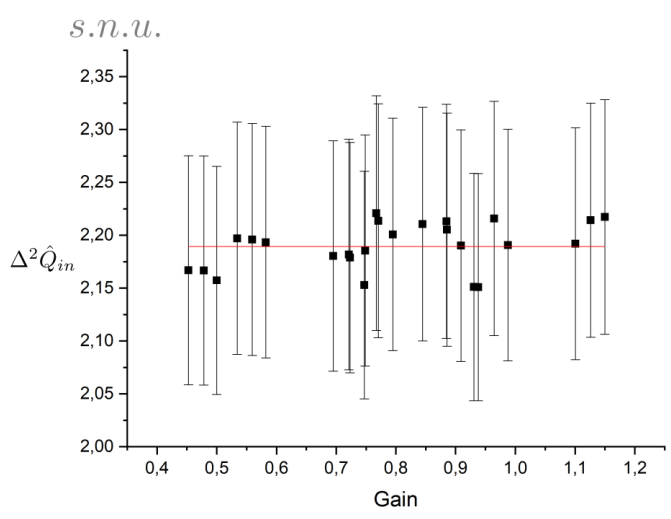

(b) Input variances of the $\hat{Q}$ quadrature.

Figure 5.27: Input variances measured by Alice as a function of $\Gamma$.

of squeezing used for the prediction are the ones modeled on the subsection (3.2.5), performed on the same day quantum teleportation results were obtained. Both squeezing and teleportation measurements were performed by maintaining the $\mathrm{OPO}$ at $0{ }^{\circ} \mathrm{C}$ under the same atmospheric conditions, explained on subsection (3.2.3).

The difference between prediction and measurement is greater for the quantum case, showing that the non-ideal gain adjustment is more harmful for quantum teleportation than it is for classical teleportation. The reason for this is the fact that antisqueezing terms are responsible for harming the measurements on the quantum case, and vacuum noise is the responsible on the classical case (eqns (4.96) and (4.97)). These graphs are to be compared with the variances of classical teleportation on fig (5.23). The values of $\Gamma$ used are the ones obtained from the classical teleportation measurements.

Fidelity results for the points $\Gamma=0.96(1)$ and $\Gamma=0.99(1)$ are shown on table (5.3) for the comparison with the uncorrected input state. The differences between fidelities measured by the cosine and by sine demodulations were greater than any other measurement as can be seen on tables (5.4) and (5.5). This is reflected on table (5.3) by the values with greater uncertainties. If the gains were defined from fig (5.25), these values of $\Gamma$ would be, respectively, $\Gamma=1.01$ and $\Gamma=1.03$.

\begin{tabular}{c|c|c|c} 
& One-mode & Symmetric combination & Antisymmetric combination \\
$\mathcal{F}(0.96)$ & $0.48(2)$ & $0.48(4)$ & $0.42(5)$ \\
$\mathcal{F}(0.99)$ & $0.42(7)$ & $0.50(3)$ & $0.37(8)$
\end{tabular}

Table 5.3: Fidelities averaged over sine and cosine demodulations.

\begin{tabular}{c|c|c|c} 
& One-mode & Symmetric combination & Antisymmetric combination \\
$\mathcal{F}(0.96)$ & $0.463(6)$ & $0.520(5)$ & $0.369(7)$ \\
$\mathcal{F}(0.99)$ & $0.352(8)$ & $0.521(5)$ & $0.292(9)$
\end{tabular}

Table 5.4: Fidelity of cosine demodulation.

\begin{tabular}{c|c|c|c} 
& One-mode & Symmetric combination & Antisymmetric combination \\
$\mathcal{F}(0.96)$ & $0.500(4)$ & $0.432(5)$ & $0.475(4)$ \\
$\mathcal{F}(0.99)$ & $0.496(4)$ & $0.471(4)$ & $0.447(3)$
\end{tabular}

Table 5.5: Fidelity of sine and demodulation. 


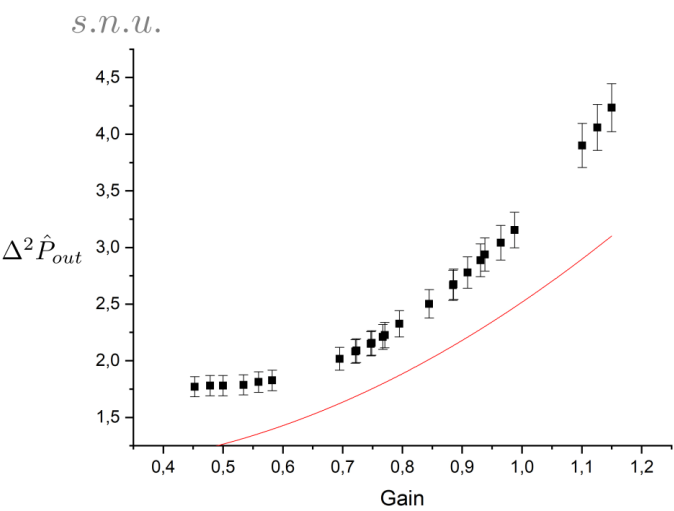

(a) Cosine demodulation.

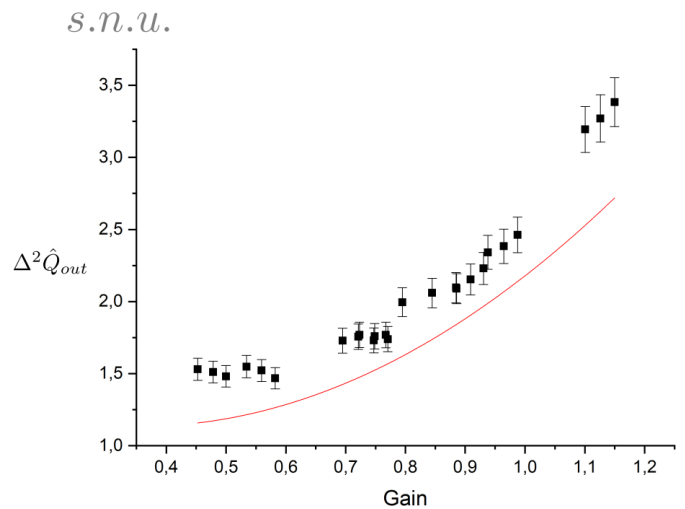

(c) Cosine demodulation.

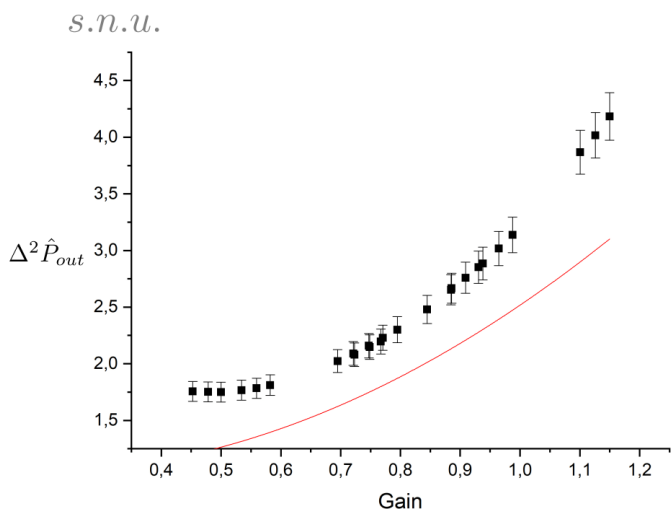

(b) Sine demodulation.

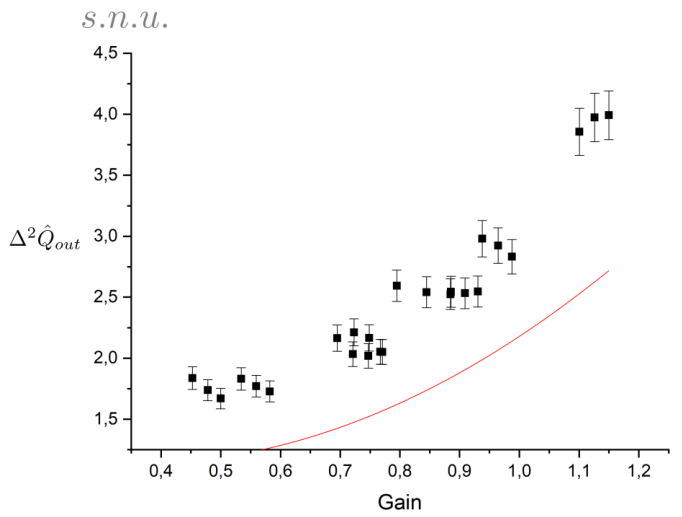

(d) Sine demodulation.

Figure 5.28: Variances of teleported state for the uncorrected input state.

Graphs showing fidelity behaviour with $\Gamma$ are shown on fig (5.29). The plotted curve is the same present on fig (4.14) with the input parameters of eqn (5.18) and the squeezing as used on fig (5.28). When the gains are defined from the classical teleportation measurements, there is a horizontal displacement between prediction and measured data, corrected when $\Gamma$ is defined from the mean values of quantum teleportation.

For the corrected input state, the results are on table (5.6). No results with $\Gamma$ greater than shown on table was measured. This table exhibits the final teleportation measurements, it is to be compared with table 5.2. It is possible to see that one-mode analysis resulted in slightly better fidelity measurements on the quantum case, however the two-mode analysis does not show big improvements.

\begin{tabular}{c|c|c|c} 
& One-mode & Symmetric combination & Antisymmetric combination \\
$\mathcal{F}(0.96)$ & $0.40(3)$ & $0.35(4)$ & $0.36(1)$ \\
$\mathcal{F}(0.98)$ & $0.392(5)$ & $0.37(3)$ & $033(2)$
\end{tabular}

Table 5.6: Fidelities averaged over sine and cosine demodulations. 


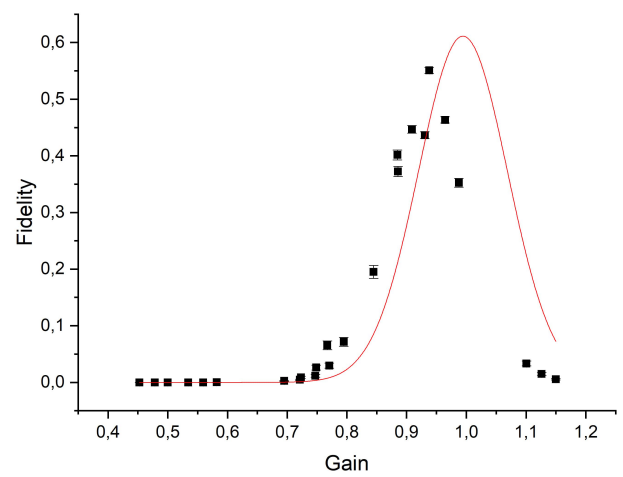

(a) Cosine demodulation. Gain defined from classical teleportation measurements.

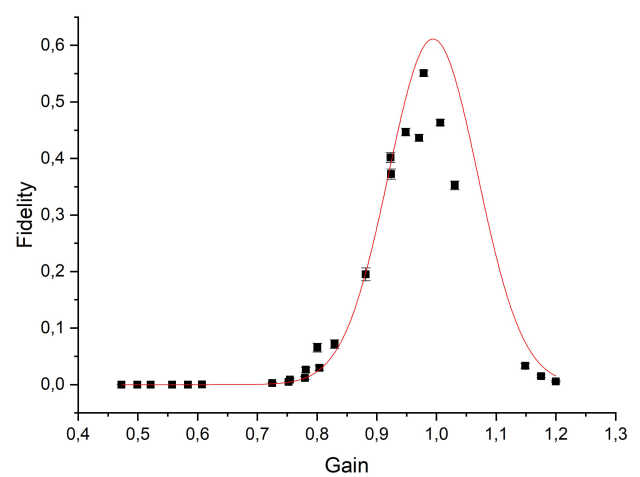

(c) Cosine demodulation. Gain defined from mean values of quantum teleportation measurements.

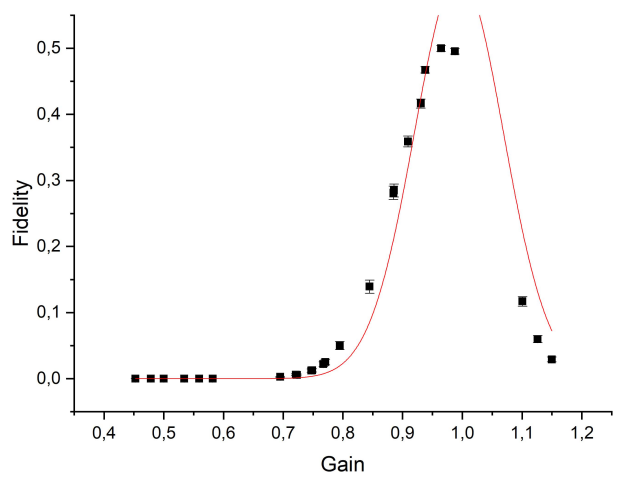

(b) Sine demodulation. Gain defined from classical teleportation measurements.

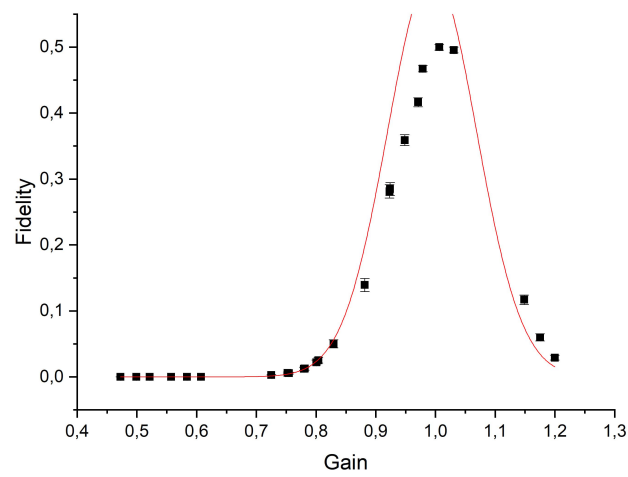

(d) Sine demodulation. Gain defined from mean values of quantum teleportation measurements.

Figure 5.29: One-mode fidelities of teleported state considering the input state as the one measured by Alice. 


\section{CONCLUSIONS AND NEXT STEPS}

The present work is a continuation of what was done by Dr. Igor Konieczniak during his Ph.D. [13]. The theoretical background for treating a more general kind of continuous variables teleportation was developed after his contribution to the system. The dimension of the Hilbert space is doubled and extra care must be taken in relation to the measurements. We used to square both sine and cosine photocurrents and sum them to retrieve $P_{M}$ and $A_{M}$ of the input state as described on section (4.2). However, when dealing with a two-mode teleportation, each demodulated signal from Alice carries different information, so a different analysis must be made. The matrix treatment of section (4.5) tells us how to use each photocurrent to retrieve all possible quadratures' combinations.

With the ability to analyse two-mode teleportation, we are able to understand the teleportation process for each sideband mode individually. Experimentally, we needed to build a new cavity to generate more general states. Since this device's reflection is frequency dependent (section (2.8)), it is possible to control the amount of energy on each sideband frequency.

The final results on table 5.6 are promising. The gain adjustment was not perfect on this day of measurements, which lead to a quantum result no greater than the classical limit. On the next days, the crystal began to present stronger gray-tracking symptoms (appendix D), preventing us from achieving better results. However, even on adverse conditions, it was achived a fidelity of $\mathcal{F}=0.40$, close to the threshold of the need for quantum resources.

There is a new crystal already in the laboratory planned to be moved to this experiment. Ph.D. student Felipe Gewers is going to lead a new project where teleportation will occur between beams with a big wavelength difference (from around $795 \mathrm{~nm}$ to around $1600 \mathrm{~nm}$ ). By using the theoretical background of this work and the experimental expertise acquired on this setup, there are no doubts results beyond the classical limit will be achieved. 


\section{APPENDICES}

\section{A Locking systems}

The six cavities and two Mach-Zehnder interferometers of the experiment are locked by three different electronic locking systems. The detailed explanation of all methods is not the goal of this section. It aims at providing an intuitive view of why they work and what is required to achieve them experimentally.

All of them make use of proportional-integral-derivative $(P I D)$ controllers. Since we want to maintain the interferometer locked at a specific set point, these devices are used to calculate the error function between the set position and the actual position and compensate for it.

\section{A .1 Side of fringe}

This is the simplest one and the most used on the experiment, locking all interferometers with the exception of the filter cavity and the OPO. For it to work, only the subtraction of two detectors and a PID controller are needed.

One of the detectors must measure a small fraction of light before it enters the interferometer, this is the reference detector. The other one measures the cavity or Mach-Zehnder output. The subtraction of the two measurements is taken for the set point to be chosen and maintained by the PID.

If the reference detector was not used, maintaining the set point stable would be a difficult task. Since this experiments takes measurements at different intensities, we would need to choose a different set point for each intensity. For example, suppose a cavity must be locked at $40 \%$ the peak's height. For different laser intensities, $40 \%$ of the peak's height represent a different different set point for each intensity. But when the reference detector is used, the set point is chosen with respect to the laser's power before the cavity. So it is the same even when the overall intensity is changed.

The PID then guarantees the position of the set point by measuring a greater or lower intensity than the established, sending the information to the PZT to increase or decrease the cavity's length. However, a problem appears when the goal is to lock at the cavity's peak. A decrease or increase in the cavity's length cannot be associated with a greater or lower intensity measurement. This happens because every position different than the set point in this case will decrease the measured intensity.

\section{A .2 Dither locking}

This is the method used for the OPO. It is very stable, but requires also a lock-in amplifier to work. This device adds a modulation of frequency $\Omega$ to the $P Z T$ - in this case, around $40 \mathrm{kHz}$. So, the measured intensity output of the cavity is $I(t)=I(L(t))=I\left(L_{0}+\Delta L \cos \Omega t\right)$, where $L$ is the cavity perimeter and $L_{0}$ is the length for which the cavity is resonant. The modulation dithers the cavity's length by $\Delta L$ with frequency $\Omega$. By Taylor expansion

$$
I(L)=I\left(L_{0}\right)+\frac{d I}{d L}\left(L_{0}\right) \Delta L \cos \Omega t+\ldots
$$

At frequency $\Omega$, the error term can be identified as

$$
e(L)=\Delta L \frac{d I}{d L}\left(L_{0}\right)
$$

a function with different signs for $\Delta L$ greater or lower than 0. By measuring the signal at this frequency and checking the sign on the obtained photocurrent, it is possible to know whether the cavity's length must be increased or decreased [51]. 


\section{A .3 Pound-Drever-Hall}

This is the method used for locking the filter cavity. Just as the dither locking method, the goal is to lock a cavity at its transmission peak and a modulation must also be used. The difference is that the modulation frequency is much higher than typically used for dither.

On this experiment, the chosen frequency is $12 \mathrm{MHz}$. A modulation such as this adds sidebands to the laser around the carrier frequency. By measuring the sidebands after being reflected by the cavity, it is possible to know whether the cavity's length must be increased or decreased [52]. This happens because the cavity treats the sidebands differently for different detunings.

This method is chosen for the first cavity on the setup. This $12 \mathrm{MHz}$ modulation is carried by the beam through all the setup. To avoid problems arising from this, every detector has filters on the $12 \mathrm{MHz}$ frequency, so these sidebands do not contaminate the information on the sidebands we are interested for the teleportation protocol.

\section{B Twin beams}

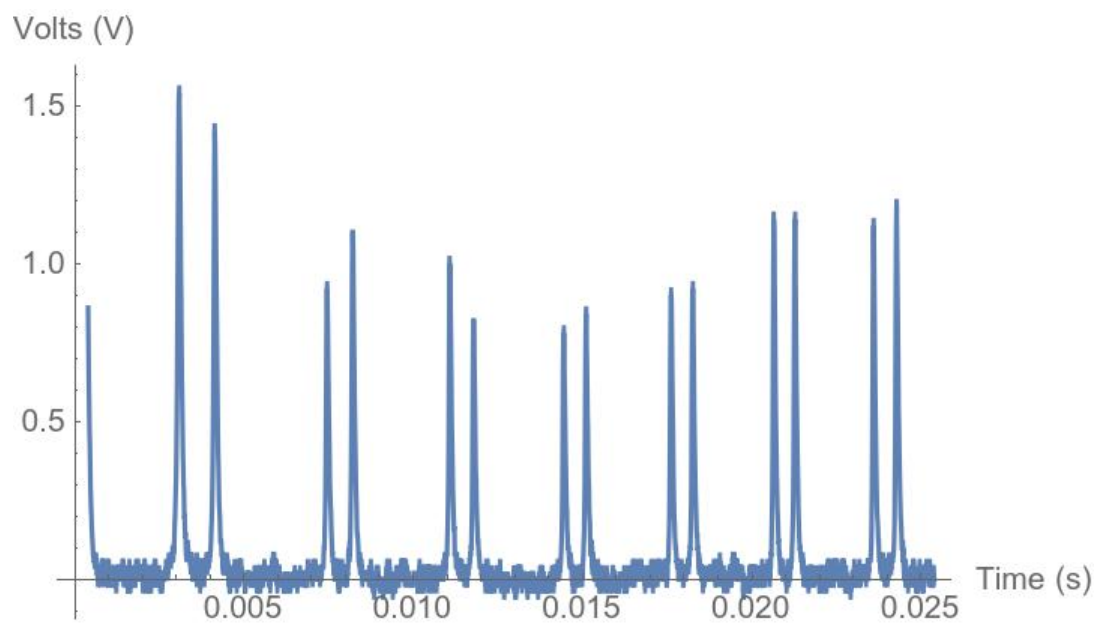

Figure 1: Both OPO outputs in tha same scanning cavity.

To measure the frequency difference between both OPO outputs, the beams must be sent into a cavity, which was left scanning at a constant speed both when increasing detuning $\Delta$ and when decreasing it. Fig (1) shows an oscilloscope measurement of the output intensity of this cavity when both beams are resonating inside as a function of the time of measure ( $t=0$ is an arbitrary time). By collecting the times which the peaks due to one of the beams (of frequency $\omega_{1}$ ) appear and the times which the peaks due to other beam (frequency $\omega_{2}$ ) appear, it is possible to find the ratio between their FSR (eqn (2.88)), giving us the ratio $\kappa$ of the beams' frequency

$$
\omega_{2}=\kappa \omega_{1}
$$

By using conservation of energy between the twin beams and the OPO pump (eqn (3.16)), which has a known frequency $\omega_{0}$, one can find the relation

$$
\hbar \omega_{0}=\hbar\left(\omega_{1}+\kappa \omega_{1}\right),
$$

yielding the desired measures for $\omega_{1}$ and $\omega_{2}$ and resulting in the wavelengths $1055 \mathrm{~nm}$ and $1072 \mathrm{~nm}$ for the OPO outputs. 


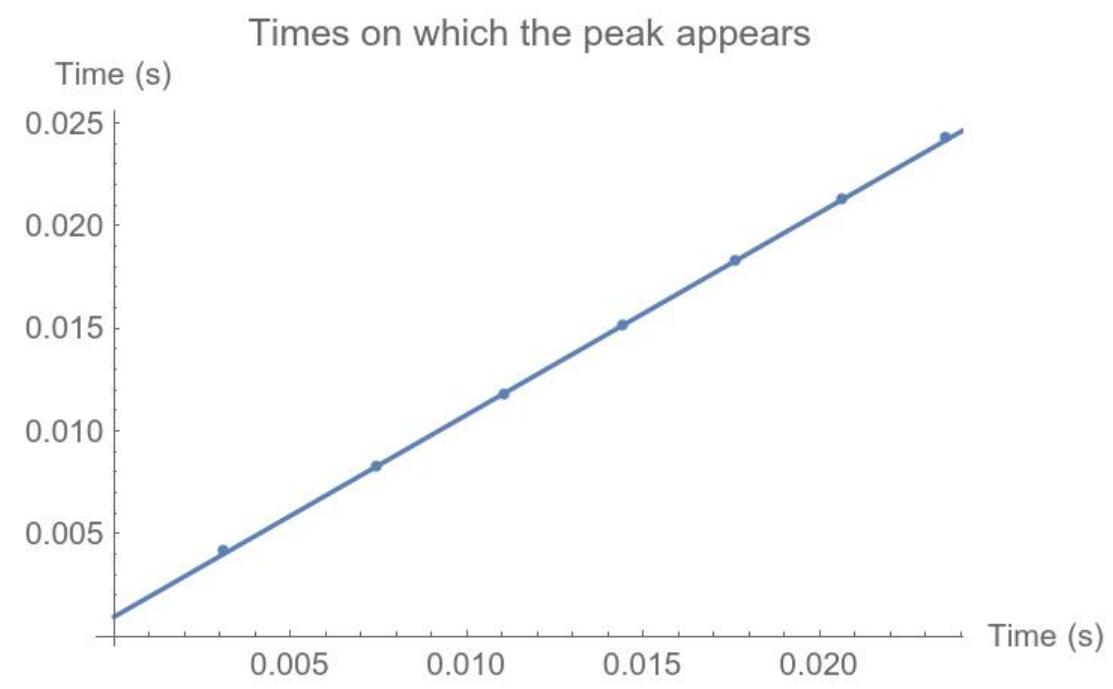

Figure 2: Plot of eqn (6.3) to the measured data exhibited on fig 1 .

\section{Modulator and demodulator not phase locked}
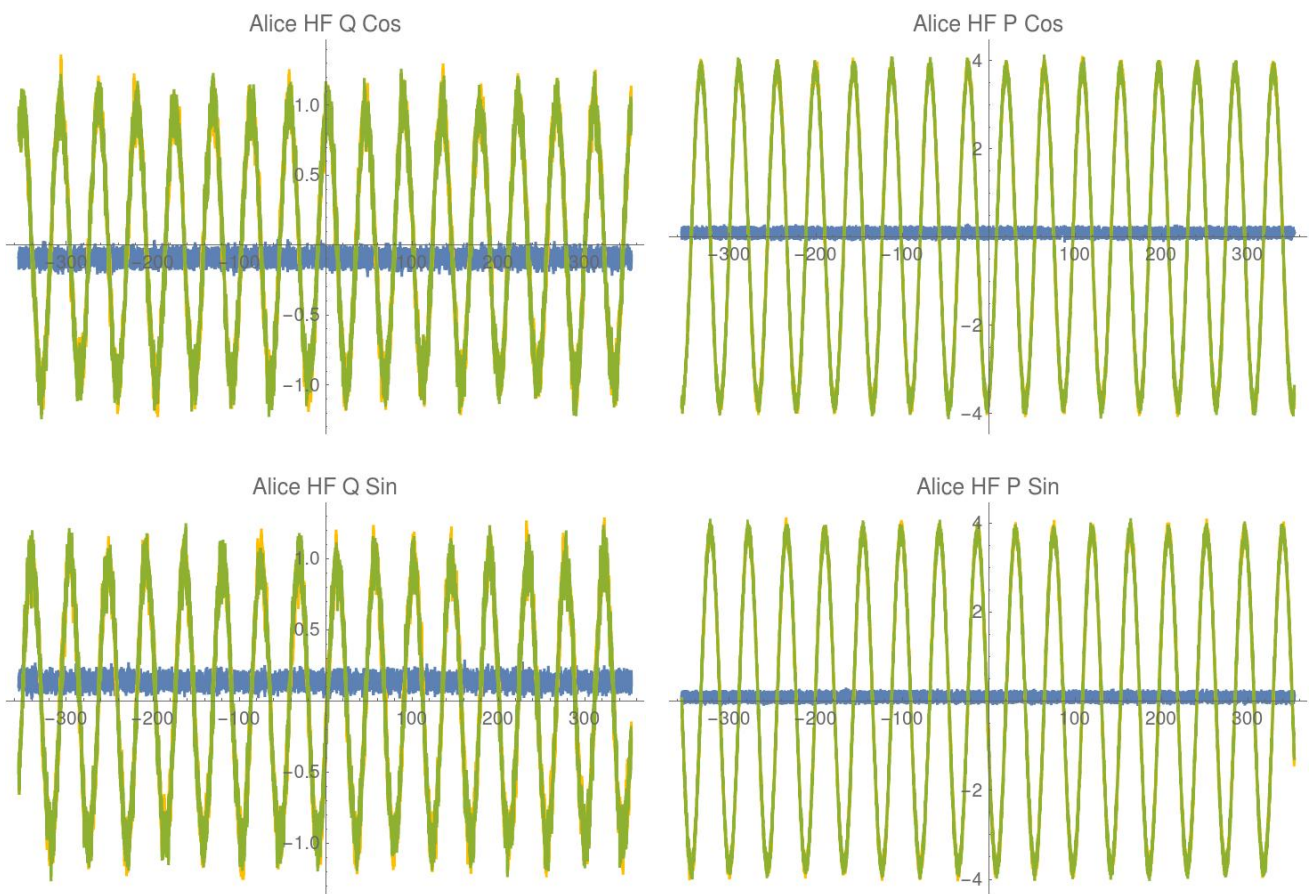

Figure 3: Alice's measurements without the use Victor's input phase modulation on the analysis.

Fig (3) shows Alice measurement results when modulation and demodulation are not phase locked between each other. The phase difference between the function generators $\left(\phi_{m}\right)$ will vary according to internal construction. Eqns (4.18) through (4.21) predict the behaviour of the outcomes when this number is not constant. These graphs can be used to infer the frequency difference between both equipment when they set at $15 \mathrm{MHz}$ as being around $20 \mathrm{~Hz}$ (16 full waves in a $750 \mathrm{~ms}$ time). 


\section{OPO stability}

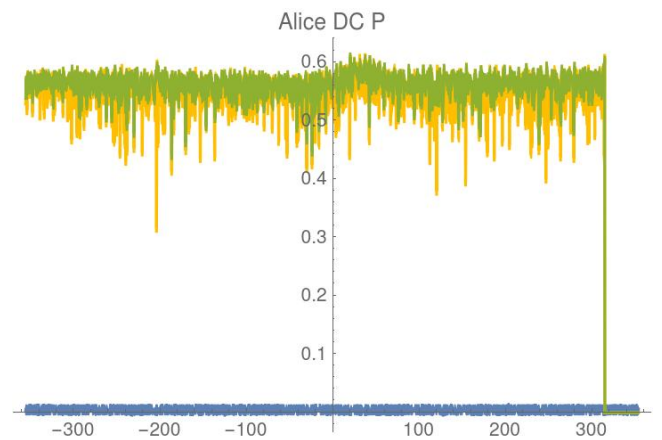

(a) Measurement from December 2018

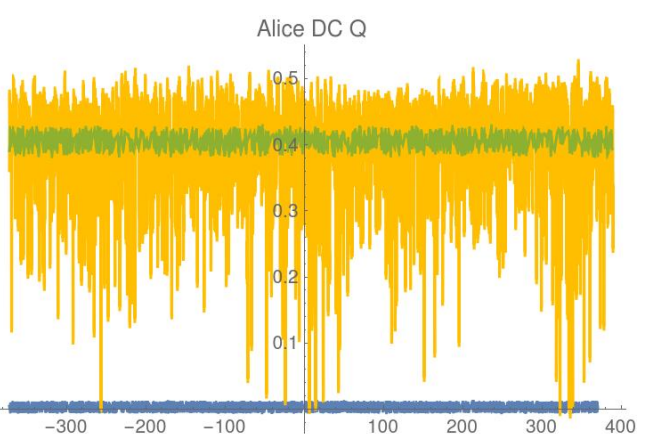

(b) Measurement from November 2019

Figure 4: Measurements with 11 months difference showing stability problemas arising on the OPO.

The biggest experimental problem while developing the work described on this thesis was to keep the OPO stable. Fig (5.3) presented on subsection (5.1.3) shows the stability difference between the OPO field and the coherent beam from Diabolo. In order to reduce the undesired fluctuations, some conditions must be controlled, such as: acoustic noise, thermal instability, gray-tracking and electronic problems.

Acoustic noise is the simplest one to solve, by choosing to take measurements when exterior sounds are absent. Thermal fluctuations are controlled by the system described on subsection (3.2.2), composed of running distilled water, two Peltiers, an aluminium foil and some copper blocks, allowing us to a temperature control on the milikelvin order.

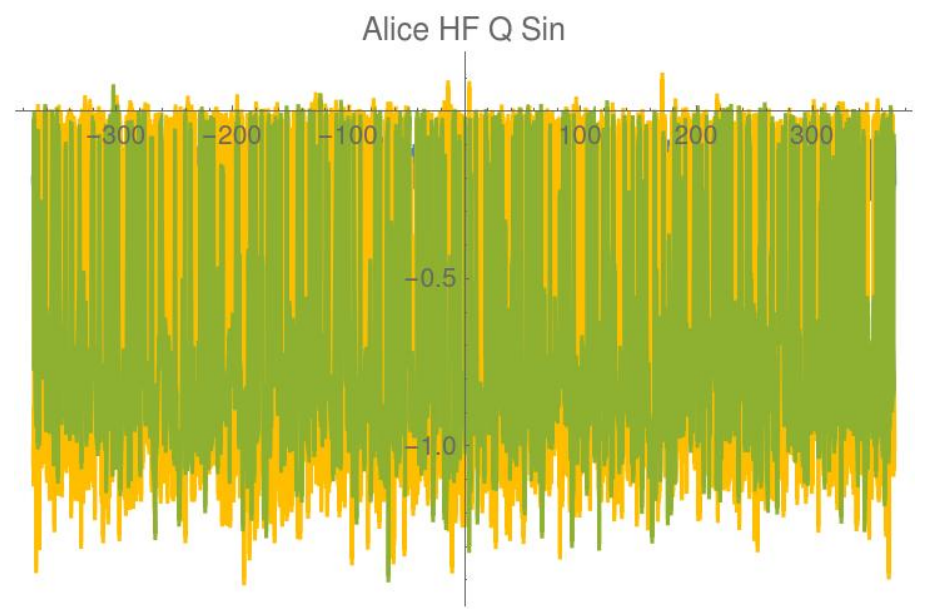

Figure 5: Example measurement of data taken after November 2019.

Gray-tracking was described on subsection (3.2.6). Since the crystal used on the experiment is being utilized since 2009 [34], the degradation generated by long term use is effective. Fig (国) shows measurements performed eleven months apart. On graph (a), the cuts explained on subsection (5.1.3) were still not necessary for the analysis, it was enough to exclude points too far from a mean value and work with what was left. After some months utilizing the crystal, the instability increased and new measurements showed results similar to graph (b) on fig (1), where the analysis required the use of data selection described on section (5.1).

The electronic problems can arise by poorly adjusted locking parameters of the dither locking method (appendix (A .2) ) used for the OPO cavity. After November 2019, all HF measurement outcomes 
Filtered APSIN
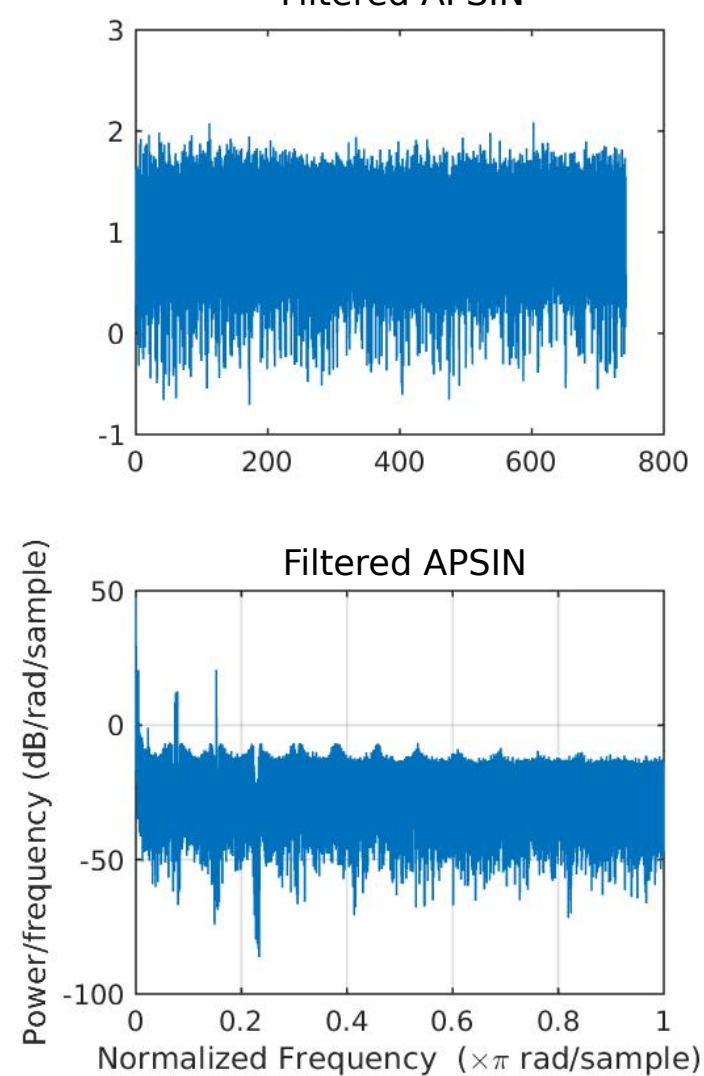
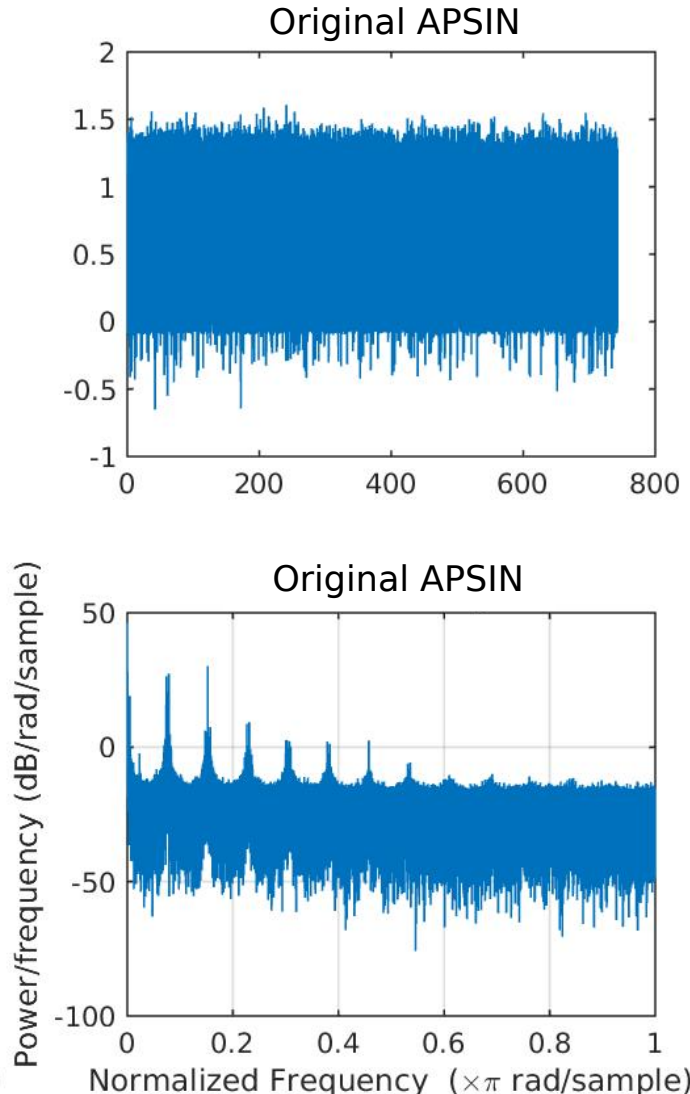

Figure 6: Notch filer application. Filtered result to the left and original photocurrent to the right. Top row shows raw data and bottom row shows data on the frequency domain.
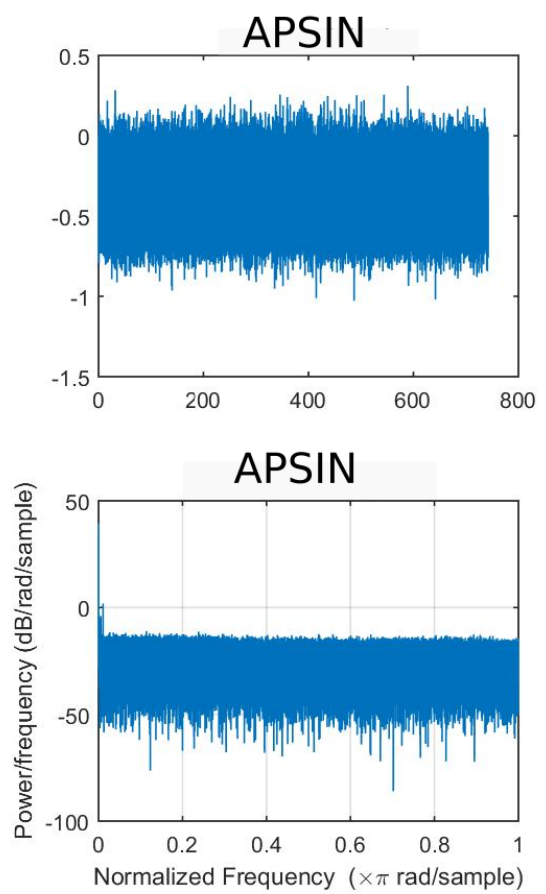

Figure 7: Top image is the $H F$ signal of a well behaving signal, bottom image is the same data on frequency domain. Since there are no peaks as fig (B), we can conclude that this effect was not present on the early measurements of this experiment. 
with entagled beams came out similar to fig (- $)$. The data is extremely noisy and data selection (subsection (5.1.3) ) was unable to solve the problem. By doing a spectral analysis on the photocurrent as shown on bottom right corner of fig (6), it was noticed that this signal contained big contributions from some specific frequencies: the frequency of the dither locking and its harmonics. We were led to conclude that the lock-in amplifier used for the locking was responsible for the harm. A digital notch filter [53] was then implemented by taking Alice's $\hat{P}$ measurement, finding the peaks on the frequency domain, and applying a narrow bandwidth filter to all data on that frequency. Comparison between filtered and original data are shown on fig (6). Before this data, measurements outcomes used to be as fig (6). So far, there are no explanations of the cause of this problem.

\section{E Classical teleportation results for other input states}

More classical teleportation results are shown on this section. One result is a teleportation performed with a state with small $\xi_{\text {in }}$ and the other is an antisymmetric state, generated by Victor locking his cavity when creating the input. In what follows, we always conside the corrected input state, with the classical limit of $\mathcal{F}=0.420$.

All the equations used for fitting are the same as the ones used on subsection (5.2). The graphs for the mean values was performed by setting one parameter free for fitting. Plots for variances and teleportation show measured data and expected results with no free parameters.

\section{E .1 Small amplitude state}

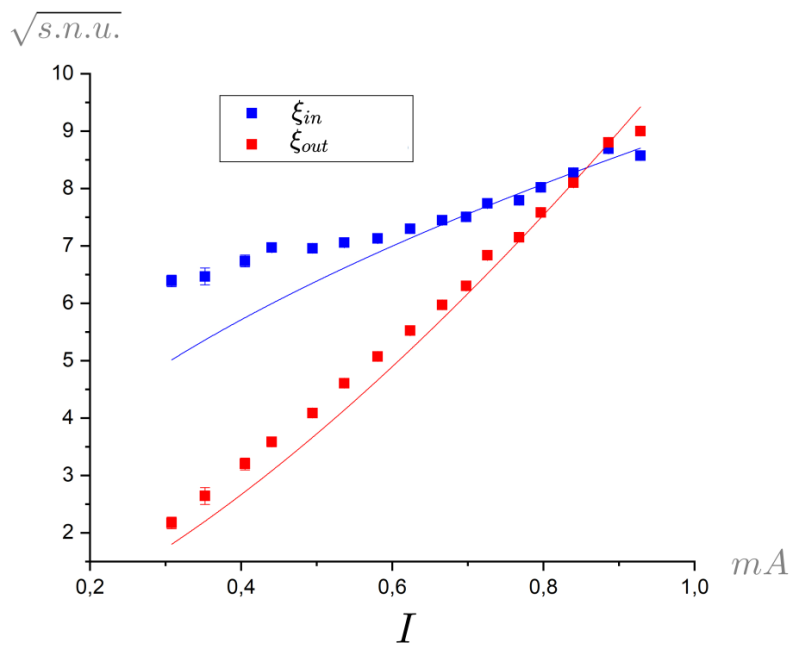

Figure 8: Amplitude of a smaller teleportation state for the corrected input state. Horizontal axis is the $D C$ photocurrent measured by the photodetector in miliamperes.

It is also interesting to study the behaviour of data with small amplitude $\xi$. Obtained measurements for a state with $\xi_{\text {in }} \approx 8.5$ are shown on fig $(8)$ for the corrected input state. The measured data closer to $\Gamma=1$ are the ones measured at $\Gamma=0.97(3)$ and $\Gamma=1.02(3)$. Quadrature values measured for $\Gamma=0.97$ are

$$
\langle\hat{P}\rangle \approx 2.7, \quad\langle\hat{Q}\rangle \approx 7.8
$$

and for the one over $\Gamma=1.02$,

$$
\langle\hat{P}\rangle \approx 8.4, \quad\langle\hat{Q}\rangle \approx 2.2 .
$$

The values of each quadrature are different for each of these values of $\Gamma$, but the amplitude $\xi$ remains consistent. This issue was caused by fluctuations on the Mach-Zehnder interferometer locking. 


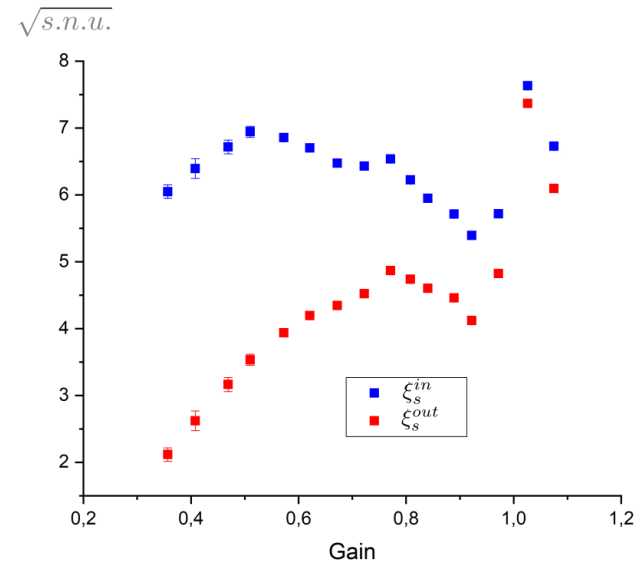

(a) $\xi_{s} \approx 5.7$.

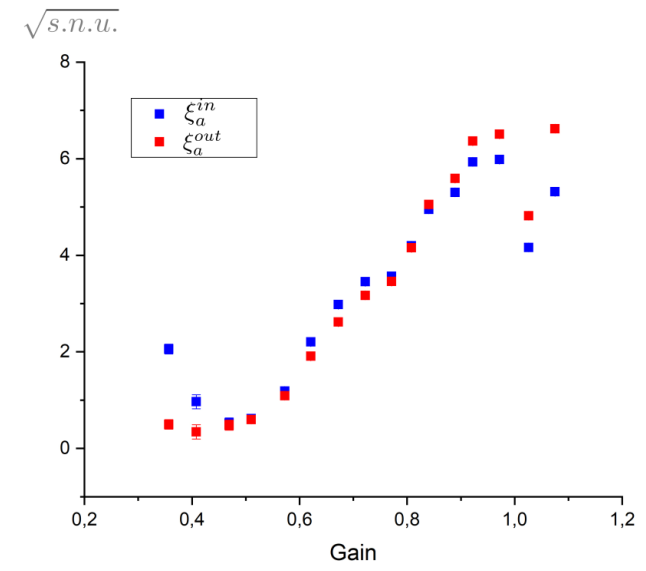

(b) $\xi_{a} \approx 6.0$.

Figure 9: Amplitude of a smaller teleportation state for the corrected input state.

Under two-mode analysis, the obtained results are the ones on fig (9). For the measurement immediately below $\Gamma=1$,

$$
\begin{array}{lll}
\left\langle\hat{p}_{s}\right\rangle \approx 2.5, & \left\langle\hat{q}_{a}\right\rangle \approx 0.9, \\
\left\langle\hat{q}_{s}\right\rangle \approx 5.1, & \left\langle\hat{p}_{a}\right\rangle \approx 5.9,
\end{array}
$$

and for the one immediately after $\Gamma=1$,

$$
\begin{array}{lll}
\left\langle\hat{p}_{s}\right\rangle \approx 7.5, & \left\langle\hat{q}_{a}\right\rangle \approx 3.9, \\
\left\langle\hat{q}_{s}\right\rangle \approx 1.5, & \left\langle\hat{p}_{a}\right\rangle \approx 1.6 .
\end{array}
$$

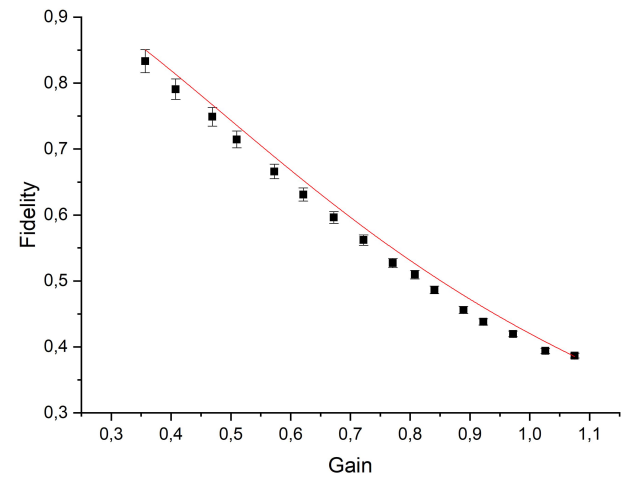

(a) Vacuum teleportation.

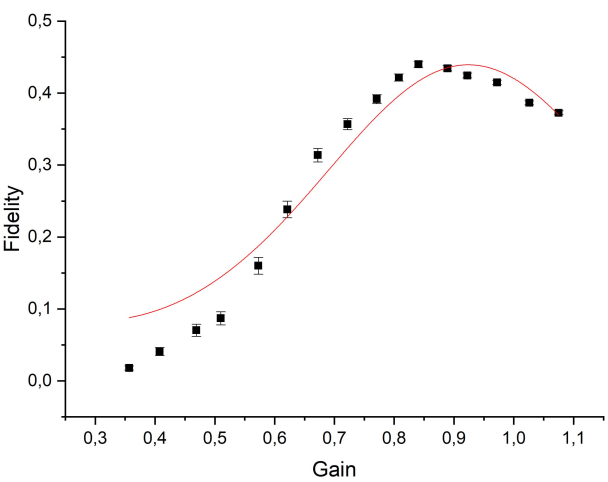

(b) Coherent state teleportation.

Figure 10: Teleportation of the small state for the corrected input state.

The quality of the variances measurements can be seen from the vacuum teleportation plot on part (a) of fig (10), where it's possible to see that a slightly better result is predicted. The effect of this deviation on the resulting fidelity may be seen on fig (b), when the mean values are considered for the calculations. The obtained fidelities are on table (A.1), averaged over sine and cosine demodulations.

Since the amplitude of the state is small, we measure values as high as $\mathcal{F}_{\text {cos }}(0.84)=0.440(4)$, greater than the classical limit of $\mathcal{F}=0.420$. As predicted on fig (4.11) for small states, fidelities bigger 


\begin{tabular}{c|c|c|c} 
& One-mode & Symmetric combination & Antisymmetric combination \\
$\mathcal{F}(0.97)$ & $0.412(3)$ & $0.361(4)$ & $0.408(3)$ \\
$\mathcal{F}(1.02)$ & $0.384(3)$ & $0.386(4)$ & $0.378(3)$
\end{tabular}

Table A.1: Small state fidelities averaged over sine and cosine demodulations.

than the classical limit may be achieved by measuring on a $\Gamma$ lesser than 1 . However, this is not a real teleportation result.

\section{E .2 Asymmetric teleportation}

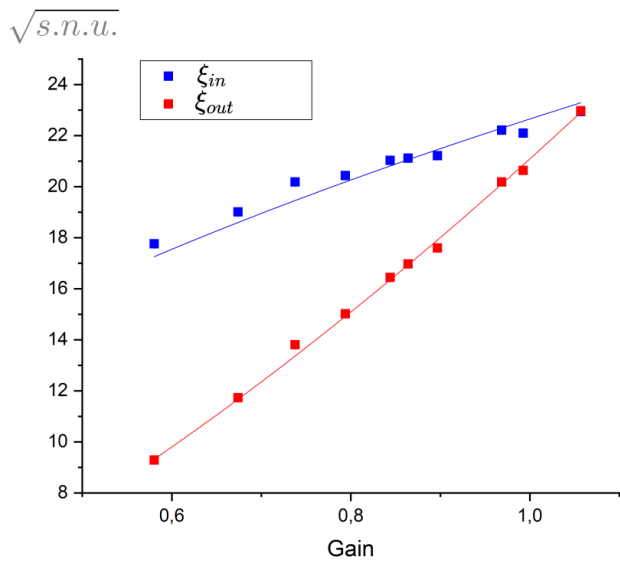

(a) One mode analysis. $\xi \approx 22$ at $\Gamma=1$.

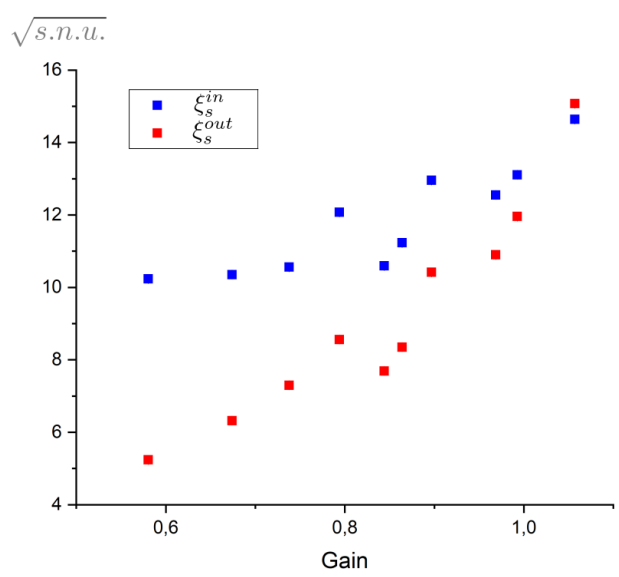

(c) Symmetric combination of quadratures.

$$
\xi_{s} \approx 13 \text { at } \Gamma=1 \text {. }
$$

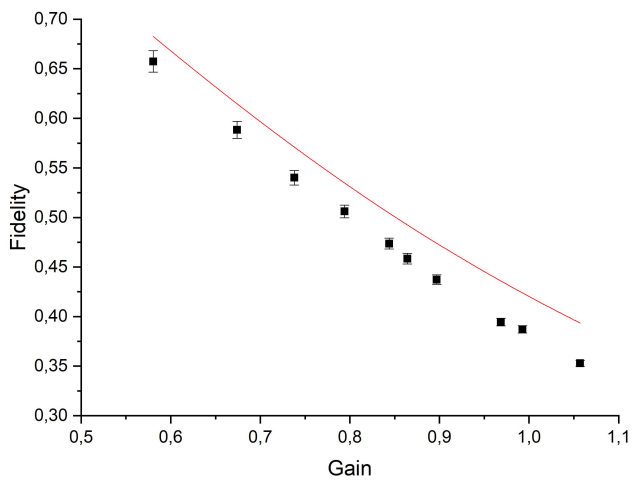

(b) Vacuum teleportation.

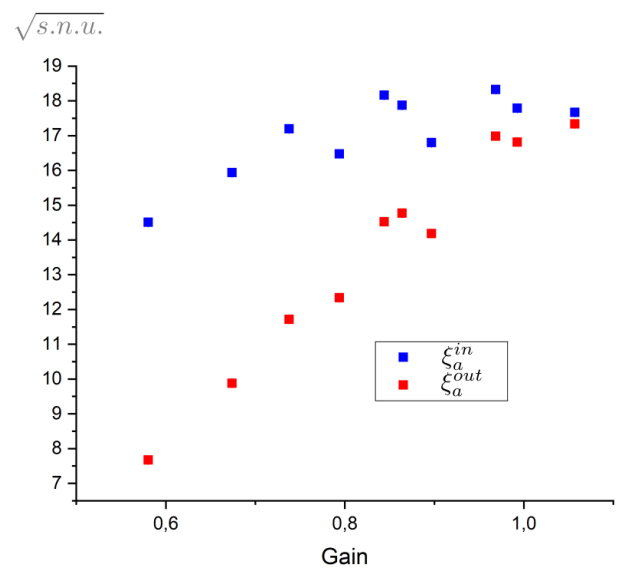

(d) Antisymmetric combination of quadratures. $\xi_{a} \approx 18$ at $\Gamma=1$.

Figure 11: Properties of asymmetrical teleported state.

Another interesting result to study is the one with properties shown on fig (11). Its mean values measurements at $\Gamma=1$ are listed below for the input state considered as the state generated by Victor

$$
\begin{array}{rlrl}
\langle\hat{P}\rangle \approx 18, & \langle\hat{Q}\rangle & \approx 12, \\
\left\langle\hat{p}_{s}\right\rangle \approx 4.1, & \left\langle\hat{q}_{a}\right\rangle \approx-18, \\
\left\langle\hat{q}_{s}\right\rangle \approx-12, & \left\langle\hat{p}_{a}\right\rangle \approx-0.2 .
\end{array}
$$

To generate this state, Victor's cavity on the input station was locked, creating an asymmetric state that could not be generated only with the Mach-Zehnder. 
Fig (12) shows results of the symmetric teleportation performed on the same day for gain adjustment. The mean values' results are as expected, being used for the definition of $\Gamma$ for all measurements of the day. Vacuum teleportation, however, seems to indicate that the gains were not so properly adjusted. This resulted on data of asymmetric teleportation of fig (11), where deviations from the expected can be seen.

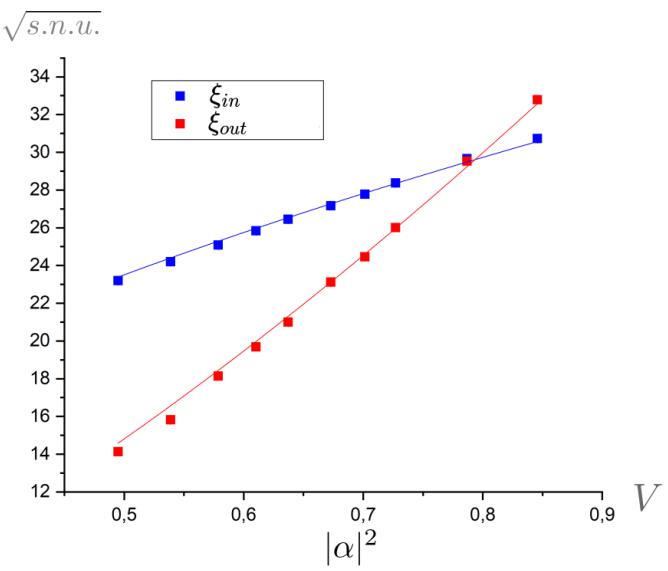

(a) Mean values.

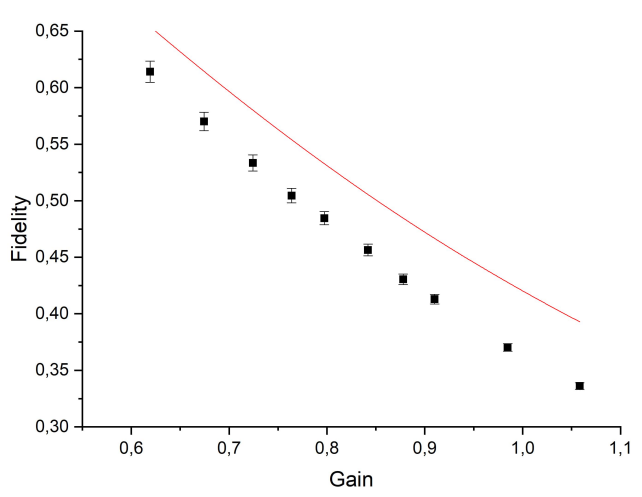

(b) Vacuum teleportation.

Figure 12: Measurements of symmetric teleportation performed on the same day.

Fidelity results for $\Gamma=0.993(3)$ and $\Gamma=1.057(3)$ are shown on table (A.2) averaged over sine and cosine demodulations.

\begin{tabular}{c|c|c|c} 
& One-mode & Symmetric combination & Antisymmetric combination \\
$\mathcal{F}(0.993)$ & $0.29(3)$ & $0.31(2)$ & $0.249(5)$ \\
$\mathcal{F}(1.057)$ & $0.348(2)$ & $0.348(2)$ & $0.319(3)$
\end{tabular}

Table A.2: Fidelities averaged over sine and cosine demodulations. 


\section{REFERENCES}

[1] D. Bouwmeester, J.-W. Pan, K. Mattle, M. Eibl, H. Weinfurter, and A. Zeilinger, "Experimental quantum teleportation," Nature, vol. 390, pp. 575-579, 121997.

[2] S. L. Braunstein and H. J. Kimble, "A posteriori teleportation," Nature, vol. 394, pp. 840-841, 8 1998.

[3] A. Furusawa, J. L. Sørensen, S. L. Braunstein, C. A. Fuchs, H. J. Kimble, and E. S. Polzik, "Unconditional quantum teleportation," Science, vol. 282, no. 5389, pp. 706-709, 1998.

[4] A. S. Villar, L. S. Cruz, K. N. Cassemiro, M. Martinelli, and P. Nussenzveig, "Generation of bright two-color continuous variable entanglement," Physical Review Letters, vol. 95, no. 24, pp. 1-4, 2005.

[5] A. S. Coelho, F. A. S. Barbosa, K. N. Cassemiro, A. S. Villar, M. Martinelli, and P. Nussenzveig, "Three-Color Entanglement," Science, vol. 326, pp. 823-826, 112009.

[6] F. A. Barbosa, A. S. Coelho, L. F. Muñoz-Martínez, L. Ortiz-Gutiérrez, A. S. Villar, P. Nussenzveig, and M. Martinelli, "Hexapartite Entanglement in an above-Threshold Optical Parametric Oscillator," Physical Review Letters, vol. 121, no. 7, pp. 1-5, 2018.

[7] Y. S. Abu-Mostafa, Neural networks and learning machines. Prentice Hall, 2009.

[8] F. Arute, K. Arya, R. Babbush, D. Bacon, J. C. Bardin, R. Barends, R. Biswas, S. Boixo, F. G. Brandao, D. A. Buell, B. Burkett, Y. Chen, Z. Chen, B. Chiaro, R. Collins, W. Courtney, A. Dunsworth, E. Farhi, B. Foxen, A. Fowler, C. Gidney, M. Giustina, R. Graff, K. Guerin, S. Habegger, M. P. Harrigan, M. J. Hartmann, A. Ho, M. Hoffmann, T. Huang, T. S. Humble, S. V. Isakov, E. Jeffrey, Z. Jiang, D. Kafri, K. Kechedzhi, J. Kelly, P. V. Klimov, S. Knysh, A. Korotkov, F. Kostritsa, D. Landhuis, M. Lindmark, E. Lucero, D. Lyakh, S. Mandrà, J. R. McClean, M. McEwen, A. Megrant, X. Mi, K. Michielsen, M. Mohseni, J. Mutus, O. Naaman, M. Neeley, C. Neill, M. Y. Niu, E. Ostby, A. Petukhov, J. C. Platt, C. Quintana, E. G. Rieffel, P. Roushan, N. C. Rubin, D. Sank, K. J. Satzinger, V. Smelyanskiy, K. J. Sung, M. D. Trevithick, A. Vainsencher, B. Villalonga, T. White, Z. J. Yao, P. Yeh, A. Zalcman, H. Neven, and J. M. Martinis, "Quantum supremacy using a programmable superconducting processor," Nature, vol. 574, no. 7779, pp. 505$510,2019$.

[9] R. P. Feynman, "Simulating physics with computers," International Journal of Theoretical Physics, vol. 21, no. 6-7, pp. 467-488, 1982.

[10] P. Shor, "Algorithms for quantum computation: discrete logarithms and factoring," in Proceedings 35th Annual Symposium on Foundations of Computer Science, pp. 124-134, IEEE Comput. Soc. Press, 2002.

[11] C. H. Bennett, G. Brassard, C. Crépeau, R. Jozsa, A. Peres, and W. K. Wootters, "Teleporting an unknown quantum state via dual classical and Einstein-Podolsky-Rosen channels," Physical Review Letters, vol. 70, pp. 1895-1899, 31993.

[12] H. J. Kimble, "The quantum internet," Nature, vol. 453, no. 7198, pp. 1023-1030, 2008.

[13] I. Konieczniak, Teletransporte de informação quântica entre campos de cores distintas. PhD thesis, USP, 2018. 
[14] T. Debuisschert, A. Sizmann, E. Giacobino, and C. Fabre, "Type-II continuous-wave optical parametric oscillators: oscillation and frequency-tuning characteristics," Journal of the Optical Society of America B, vol. 10, p. 1668, 91993.

[15] F. A. S. Barbosa, A. S. Coelho, K. N. Cassemiro, P. Nussenzveig, C. Fabre, M. Martinelli, and A. S. Villar, "Beyond Spectral Homodyne Detection: Complete Quantum Measurement of Spectral Modes of Light," Physical Review Letters, vol. 111, p. 200402, 112013.

[16] F. A. S. Barbosa, A. S. Coelho, K. N. Cassemiro, P. Nussenzveig, C. Fabre, A. S. Villar, and M. Martinelli, "Quantum state reconstruction of spectral field modes: Homodyne and resonator detection schemes," Physical Review A, vol. 88, p. 052113, 112013.

[17] D. J. Griffiths, Introduction to Electrodynamics. Prentice Hall, 1999.

[18] M. O. Scully and M. S. Zubairy, Quantum Optics, vol. 1. Cambridge University Press, 91997.

[19] T. P. Pearsall, Quantum Photonics, vol. 60 of Graduate Texts in Physics. Cham: Springer International Publishing, 2017.

[20] G. Grynberg, A. Aspect, and C. Fabre, Introduction to Quantum Optics. Cambridge University Press, 2010.

[21] A. Ferraro, M. G. A. Paris, and S. Olivares, Gaussian states in quantum information. No. Bibliopolis 01, 2005.

[22] W. P. Schleich, Quantum Optics in Phase Space. WILEY-VCH, 2001.

[23] C. Cohen-Tannoudji, C. Cohen-Tannoudji, J. Dupont-Roc, J. Dupont-Roc, G. Grynberg, and G. Grynberg, Photons and Atoms - Introduction to Quantum Electrodynamics. Wiley Professional, 1997.

[24] L. F. Cohen-Tannoudji C., Diu B., Quantum mechanics. Wiley-VCH, 1996.

[25] D. J. Griffiths, Introduction to Quantum Mechanics. Prentice Hall, 1994.

[26] G. Adesso, S. Ragy, and A. R. Lee, "Continuous Variable Quantum Information: Gaussian States and Beyond," Open Systems \& Information Dynamics, vol. 21, p. 1440001, 62014.

[27] E. Wigner, "On the quantum correction for thermodynamic equilibrium," Physical Review, vol. 40, no. 5, pp. 749-759, 1932.

[28] H. A. Bachor, "A Guide to Experiments in Quantum Optics," 2004.

[29] A. D. S. Villar, Emaranhamento Multicolor entre Feixes Intensos de Luz. PhD thesis, USP, 2007.

[30] F. A. S. Barbosa, Robustez do emaranhamento em variáveis contínuas e fotodetecção de feixes intensos no domínio temporal. PhD thesis, USP, 2013.

[31] R. B. d. Andrade, An optical parametric oscillator for a light-atomic media interface. PhD thesis, USP, 2018.

[32] B. Saleh, Fundamentals of Photonics. John Wiley \& Sons, Inc., 1991.

[33] A. S. Villar, "The conversion of phase to amplitude fluctuations of a light beam by an optical cavity," American Journal of Physics, vol. 76, no. 10, pp. 922-929, 2008. 
[34] A. S. O. Coelho, Emaranhamento multicor para redes de informação quântica. PhD thesis, USP, 2013.

[35] L. Mandel and E. Wolf, Optical Coherence and Quantum Optics. Cambridge University Press, 1995.

[36] S. Cialdi, C. Porto, D. Cipriani, S. Olivares, and M. G. Paris, "Full quantum state reconstruction of symmetric two-mode squeezed thermal states via spectral homodyne detection and a state-balancing detector," Physical Review A, vol. 93, no. 4, pp. 1-7, 2016.

[37] A. Einstein, B. Podolsky, and N. Rosen, "Can Quantum-Mechanical Description of Physical Reality Be Considered Complete?," Physical Review, vol. 47, pp. 777-780, 51935.

[38] J. S. Bell, "On the Einstein Podolsky Rosen paradox," Physics Physique Fizika, vol. 1, pp. 195-200, 111964.

[39] M. D. Reid, "Demonstration of the Einstein-Podolsky-Rosen paradox using nondegenerate parametric amplification," Physical Review A, vol. 40, pp. 913-923, 71989.

[40] L.-M. Duan, G. Giedke, J. I. Cirac, and P. Zoller, "Inseparability Criterion for Continuous Variable Systems," Physical Review Letters, vol. 84, pp. 2722-2725, 32000.

[41] J. E. César, A. S. Coelho, K. N. Cassemiro, A. S. Villar, M. Lassen, P. Nussenzveig, and M. Martinelli, "Extra phase noise from thermal fluctuations in nonlinear optical crystals," Physical Review A Atomic, Molecular, and Optical Physics, vol. 79, no. 6, pp. 1-11, 2009.

[42] M. Martinelli, "Compressão de ruído quântico e efeitos transversos em osciladores paramétricos ópticos," (Tese), p. 246, 2002.

[43] B. Boulanger, I. Rousseau, J. P. Fève, M. Maglione, B. Ménaert, and G. Marnier, "Optical Studies of Laser-Induced Gray-Tracking in KTP," IEEE Journal of Quantum Electronics, vol. 35, no. 3, pp. 281-286, 1999 .

[44] M. Nielsen and I. Chuang, Quantum Computation and Quantum Information. Cambridge University Press, 2011.

[45] S. L. Braunstein and H. J. Kimble, "Teleportation of Continuous Quantum Variables," Physical Review Letters, vol. 80, pp. 869-872, 11998.

[46] P. van Loock, S. L. Braunstein, and H. J. Kimble, "Broadband teleportation," Physical Review A Atomic, Molecular, and Optical Physics, vol. 62, no. 2, p. 18, 2000.

[47] T. C. Zhang, K. W. Goh, C. W. Chou, P. Lodahl, and H. J. Kimble, "Quantum teleportation of light beams," Physical Review A, vol. 67, no. 3, p. 033802, 2003.

[48] S. L. Braunstein, C. A. Fuchs, and H. J. Kimble, "Criteria for continuous-variable quantum teleportation," Journal of Modern Optics, vol. 47-2, no. 3, pp. 267-278, 2000.

[49] F. Grosshans and P. Grangier, "Quantum cloning and teleportation criteria for continuous quantum variables," Physical Review A - Atomic, Molecular, and Optical Physics, vol. 64, no. 1, p. 4, 2001.

[50] W. P. Bowen, N. Treps, B. C. Buchler, R. Schnabel, T. C. Ralph, T. Symul, and P. K. Lam, "Unity Gain and Nonunity Gain Quantum Teleportation," IEEE Journal on Selected Topics in Quantum Electronics, vol. 9, no. 6, pp. 1519-1532, 2003.

[51] F. Herzog, K. Kudielka, D. Erni, and W. Bächtold, "Optical phase locking by local oscillator phase dithering," IEEE Journal of Quantum Electronics, vol. 42, no. 10, pp. 973-985, 2006. 
[52] R. W. P. Drever, J. L. Hall, F. V. Kowalski, J. Hough, G. M. Ford, A. J. Munley, and H. Ward, "Laser phase and frequency stabilization using an optical resonator," Applied Physics B Photophysics and Laser Chemistry, vol. 31, pp. 97-105, 61983.

[53] Q. Wang and D. Kundur, "A generalized design framework for IIR digital multiple notch filters," Eurasip Journal on Advances in Signal Processing, vol. 2015, no. 1, 2015. 